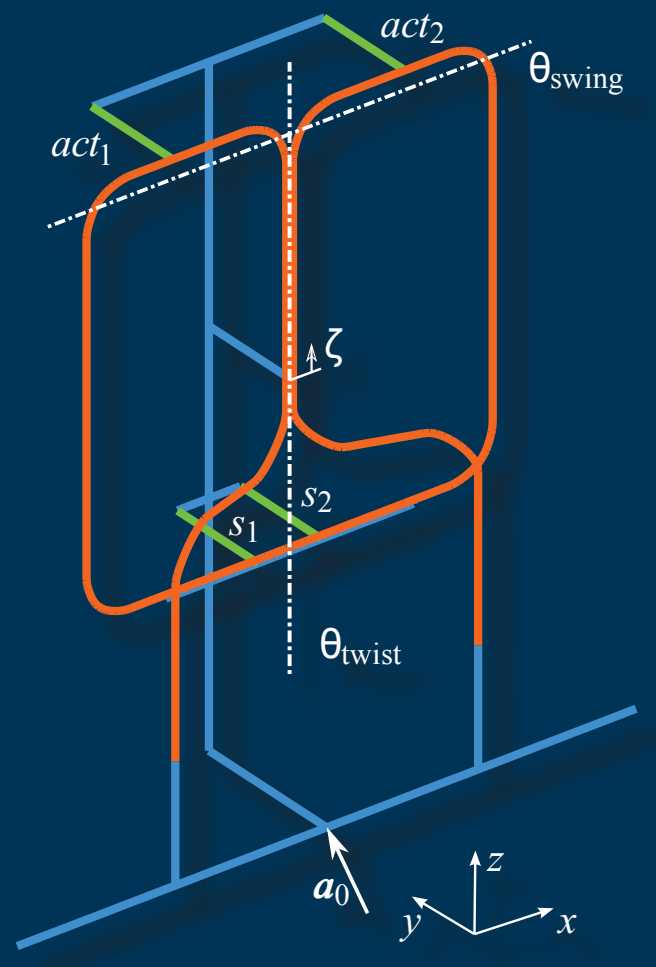

\title{
Vibration Isolation for Coriolis Mass-Flow Meters
}

\section{Bert van de Ridder PhD Thesis}





\section{VIBRATION ISOLATION FOR CORIOLIS MASS-FLOW METERS}

L. (BERT) VAN DE RIDDER 


\section{Composition of the Graduation Committee:}

Chairman and secretary:

prof.dr. G.P.M.R. Dewulf University of Twente

\section{Promotor:}

prof.dr.ir. A. de Boer

University of Twente

Co-promotors:

dr.ir. J. van Dijk

University of Twente

dr.ir. W.B.J. Hakvoort

University of Twente \& DEMCON Advanced Mechatronics

\section{Members:}

prof.dr.ir. M. Verhaegen prof.dr.ir. A. Preumont prof.dr.ir. J.C. Lötters prof.dr.ir. H. van der Kooij prof.dr.ir. L. Abelmann
Delft University of Technology

Université Libre de Bruxelles

University of Twente \& Bronkhorst High-Tech BV

University of Twente \& Delft University of Technology

University of Twente \& KIST Europe

This work was performed at the Laboratory of Mechanical Automation and Mechatronics, Department of Mechanics, Solids, Surfaces \& Systems $\left(\mathrm{MS}^{3}\right)$, Faculty of Engineering Technology, University of Twente, P.O. Box 217, 7500 AE Enschede, the Netherlands.

This research was financed by the support of the Dutch association Pieken in de Delta Programme, project PID092051, of the Dutch Ministry of Economic Affairs.

On the cover: a SPACAR model of a CMFM and on the back a functional model of a CMFM with integrated active vibration control.

Vibration Isolation for Coriolis Mass-Flow Meters

Lubbert van de Ridder

Email: bert.vdridder@gmail.com

PhD Thesis, University of Twente, Enschede, the Netherlands

ISBN 978-90-365-3874-9

DOI 10.3990/1.9789036538749

Copyright (C) June 2015 by L. van de Ridder, the Netherlands

Printed by: Ipskamp Drukkers - Enschede 


\title{
VIBRATION ISOLATION FOR CORIOLIS MASS-FLOW METERS
}

\section{DISSERTATION}

\author{
to obtain \\ the degree of doctor at the University of Twente, \\ on the authority of the rector magnificus, \\ prof.dr. H. Brinksma, \\ on account of the decision of the graduation committee, \\ to be publicly defended \\ on Wednesday the $24^{\text {th }}$ of June, 2015 at 16:45
}

by

\section{LUBBERT VAN DE RIDDER}

born on the $22^{\text {nd }}$ of April, 1987

in Putten, the Netherlands 
This thesis has been approved by the promotor

prof.dr.ir. A. de Boer

and the assistant-promotors

dr.ir. J. van Dijk

dr.ir. W.B.J. Hakvoort 


\section{Summary}

A Coriolis Mass-Flow Meter (CMFM) is an active device based on the Coriolis force principle for direct mass-flow measurements, with high accuracy, range-ability and repeatability. The working principle of a CMFM is as follows: a fluid conveying tube is actuated to oscillate at a low amplitude, whereby a resonance frequency is used to minimise the amount of required energy. A fluid-flow in the vibrating tube induces Coriolis forces, which are proportional to the mass-flow, and affect the tube motion resulting in a change of the mode shape. Measuring the tube displacement in such a way that the change of its mode shape is determined, allows calculating the mass-flow.

External vibrations create additional components in the CMFM sensor signals and such additional components can introduce a measurement error. For low flows $(<1 \mathrm{~kg} / \mathrm{h})$, the Coriolis force induced motion is relatively small compared to motions induced by external vibrations, thus CMFMs designed to be sensitive to low flows are rather sensitive to external vibrations.

Mainly external vibrations around the meter's drive and Coriolis frequencies produce a measurement error, regardless of the phase detection algorithm used. A model-based, quantitative estimation of the expected mass-flow error in response to external vibrations is obtained from the transmissibility function of external vibrations to the tube displacement measurement.

Narrow-band vibration isolation is required to improve the sensor performance. In this thesis the sensitivity to external vibrations is reduced, using passive and active vibration isolation solutions. Passive vibration isolation consists of a mass-spring-damper system between an excitation source and an influenced receiver (e.g. the floor and the measurement device).

The performance is insufficient, because the suspension frequency is limited by the maximum stress in the connection tubes and a maximum allowable sag due to gravity. Therefore the passively suspended stage is extended with voice-coil actuators and absolute motion sensors to apply active vibration isolation control. Feedback and feed-forward control algorithms are compared. Increased narrow-band vibration attenuation is achieved using loop shaping feedback control. Alternatively, an innovative feed-forward strategy is used, where by the parameters, which are tuned using an adaptive FxLMS technique, are based on the damping and stiffness properties of the stage. 
A second alternative concept is also presented; an absolute tube displacement measurement is achieved by balancing the displacement sensor. The sensitivity for external vibrations is reduced by tuning the resonance frequency and damping ratio of the balancing mechanism to those of the tube. Active control is added to compensate for the tube dynamics variation due to the different fluid densities.

Based on the theoretic models, two proof of principles mechanisms are realised for validation. Attenuation of more than $40 \mathrm{~dB}$ (factor 100) is achieved for a CMFM with integrated active vibration isolation for both the feedback and feed-forward scheme. The performance is limited by sensor noise and for the feedback solution by the destabilising effect of higher order dynamics. The balanced sensor mechanism shows another $24 \mathrm{~dB}$ (factor 16) reduction, which is limited by the higher order dynamics of the fluid conveying tube.

The thesis presents a comprehensive analysis on how vibration isolation can be achieved. It is mainly focussed on narrow-band vibration isolation applied to a CMFM, but not limited thereto. Active vibration isolation control is successfully implemented in a design of a CMFM, for the first time. A reduced sensitivity for external vibrations revealed other dependencies of disturbances on the measurement value. Future research is needed to identify those parameters affecting the accuracy and precision of a CMFM and to increase robustness for parameter changes. 


\section{Samenvatting}

Een Coriolis Massa-Flow Meter (CMFM) is een actief instrument, gebaseerd op het Coriolis effect, voor een rechtstreekse meting van de massastroom. De meting heeft een hoge nauwkeurigheid, een groot bereik en een hoge herhaalbaarheid. De werking van een CMFM is als volgt: een met fluïdum (vloeistof of gas) gevulde buis wordt geactueerd, zodat deze gaat resoneren met een kleine amplitude op zijn eigenfrequentie. Een bewegend fluïdum in de trillende buis zorgt voor Coriolis krachten, welke evenredig zijn met de massastroom. Deze hebben een effect op de buisbeweging, zodanig dat de trilvorm verandert. Het meten van de buisverplaatsingen, zodanig dat de verandering van de trilvorm kan worden bepaald, maakt het mogelijk om de massastroom te bepalen.

Externe trillingen resulteren in extra componenten in de CMFM sensorsignalen. Deze extra componenten kunnen leiden tot een meetfout. Voor instrumenten met een klein doorstroombereik $(<1 \mathrm{~kg} / \mathrm{h})$ is de beweging ten gevolge van de Coriolis kracht relatief klein vergeleken met de beweging ten gevolge van externe trillingen. Daarom is een CMFM, welke gevoelig is voor een kleine massatroom, erg gevoelig voor externe trillingen.

Voornamelijk externe trillingen rond de aandrijf en Coriolis frequentie resulteren in een meetfout, onafhankelijk van het gebruikte algoritme om het faseverschil te bepalen. Er is een model gerealiseerd om de meetfout ten gevolge van externe trillingen kwantiatief te kunnen schaten. Deze schatting is gebaseerd op de overdrachtsfunctie van externe trillingen naar de gemeten buisverplaatsingen.

Om de gevoeligheid voor externe trillingen te verminderen is trillingsisolatie in een klein frequentiegebied noodzakelijk. In dit proefschrift is de gevoeligheid voor externe trillingen verkleind door gebruik te maken van passieve en actieve trillingsisolatieoplossingen. De passieve trillingsisolatieoplossing bestaat uit een massa-veer-demper systeem tussen de externe trillingsbron en de ontvanger. (In het beschouwde geval, de vloer en het meetinstrument).

De onderdrukking is onvoldoende, omdat de frequentie van de afgeveerde platform wordt beperkt door de maximaal toelaatbare spanning in de aansluitbuizen en de maximale zakking ten gevolgde van de zwaartekracht. Daarom is de passieve platform uitgebreid met voice-coil actuatoren en absolute bewegingsensoren om actieve trillingsisolatie toe te kunnen passen. Een terugkoppel en een vooruitkoppelstrategie zijn met elkaar vergeleken. Trillingsisolatie 
in een klein frequentiegebied is verkregen door de terugkoppelregelaar versterking in dit frequentiegebied te maximaliseren. Een innovatieve vooruitkoppelstrategie is geïmplementeerd, waarbij de parameters worden bijgesteld met behulp van een FxLMS algoritme. De parameters zijn afhankelijk van de demping en de stijfheid tussen de behuizing en de afgeveerde platform.

Verder is een tweede alternatief concept gepresenteerd. Een absolute meting van de buisverplaatsing is verkregen door het balanceren van de verplaatsingssensor. De gevoeligheid voor externe trillingen is verminderd door het aanpassen van de resonantiefrequentie en de dempingsratio van het gebalanceerde mechanisme aan die van de buis. Actieve elementen zijn toegevoegd om te kunnen compenseren voor de veranderingen in de buisdynamica, bijvoorbeeld ten gevolge van een andere dichtheid van het fluïdum.

Gebaseerd op de theoretische modellen zijn twee opstellingen gerealiseerd voor de validatiestap. Een onderdrukking van meer dan $40 \mathrm{~dB}$ (een factor 100) is gerealiseerd met de experimentele opstelling voor de terugkoppel en de vooruitkoppelstategie. De onderdrukking wordt beperkt door de ruis van de acceleratiesensoren en voor de terugkoppelstrategie tevens door het destabiliserende effect van de hogere orde dynamica van de afgeveerde platform. De opstelling met de gebalanceerde sensor laat een onderdrukking van $24 \mathrm{~dB}$ (een factor 16) zien. Deze wordt beperkt door de hogere orde dynamica van buis.

Het proefschrift presenteert een uitgebreide analyse,die laat zien hoe trillingsisolatie in combinatie met een CMFM kan worden gerealiseerd. De strategie is voornamelijk gericht op het verkleinen van de trillingsgevoeligheid in een klein frequentiegebied. Actieve trillingsisolatie is voor het eerst, succesvol geïmplementeerd in het ontwerp van een CMFM. Een gereduceerde gevoeligheid voor externe trillingen, laat zien dat dat er nu andere verstoringen dominant zijn. Dit is onderwerp voor toekomstig onderzoek. 


\section{Contents}

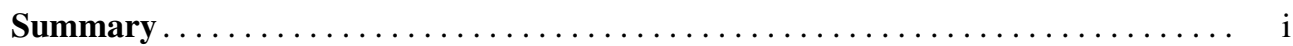

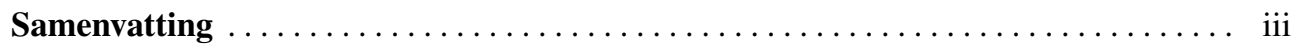

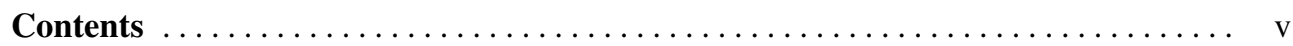

Nomenclature $\ldots \ldots \ldots \ldots \ldots \ldots \ldots \ldots \ldots \ldots \ldots \ldots \ldots \ldots$ vii

\section{Part I Thesis}

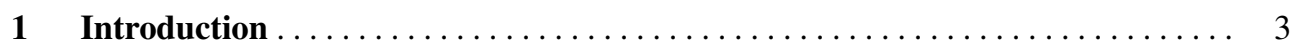

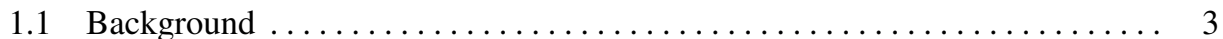

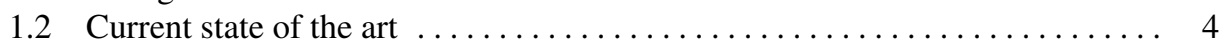

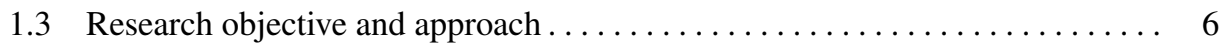

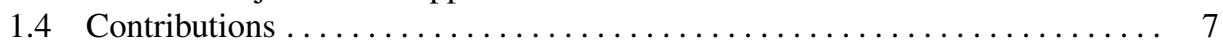

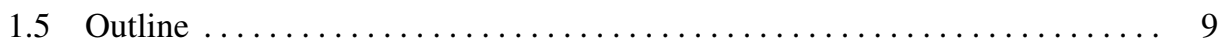

2 Ouantification of the influence of external vibrations $\ldots \ldots \ldots \ldots \ldots \ldots \ldots \ldots$

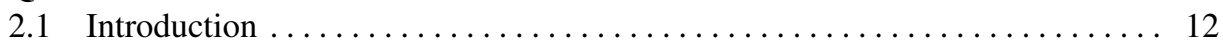

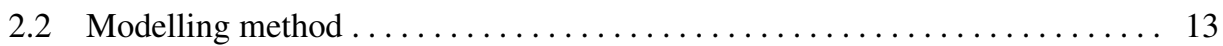

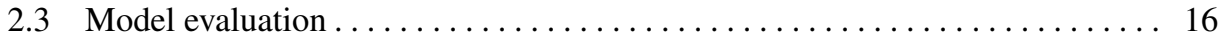

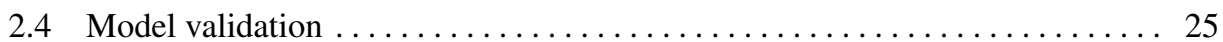

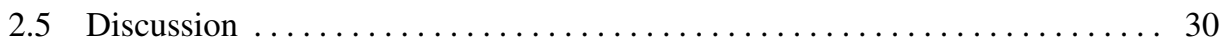

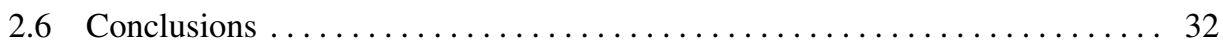

$3 \quad$ Active vibration isolation feedback control $\ldots \ldots \ldots \ldots \ldots \ldots \ldots \ldots \ldots$

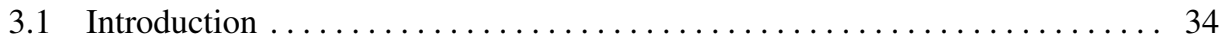

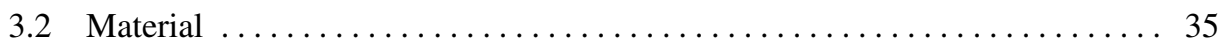

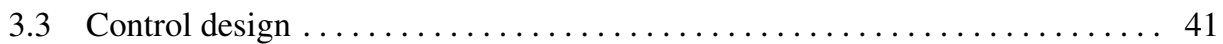

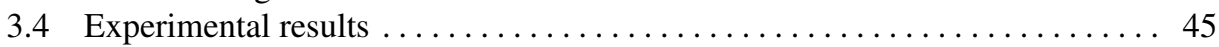

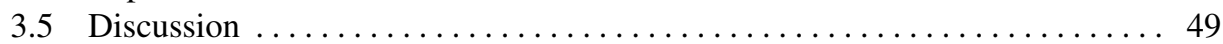




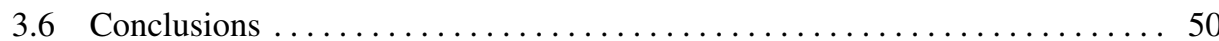

$4 \quad$ CMFM with integrated Active Vibration Isolation Control . . . . . . . . . . . . 51

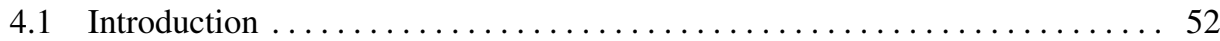

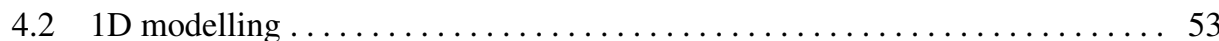

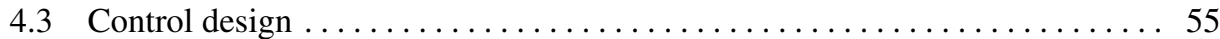

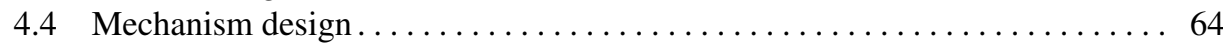

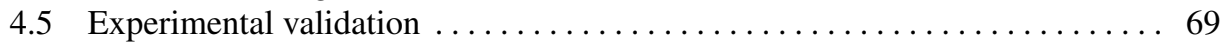

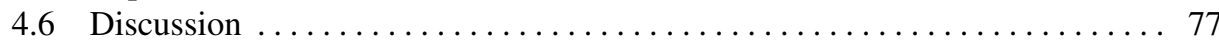

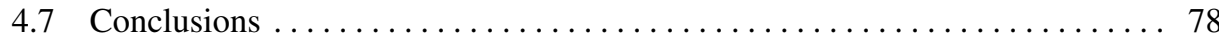

5 Vibration isolation by compliant sensor mounting $\ldots \ldots \ldots \ldots \ldots \ldots \ldots \ldots$

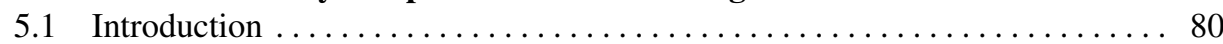

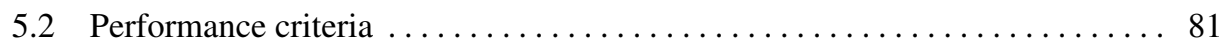

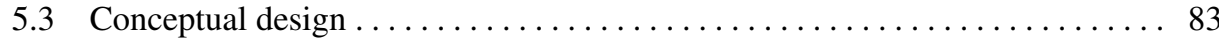

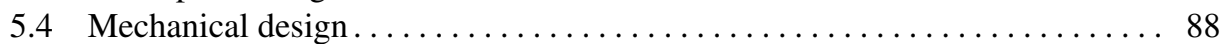

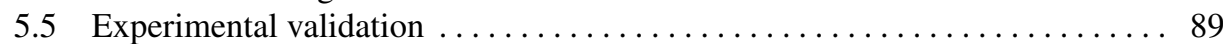

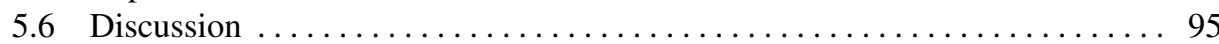

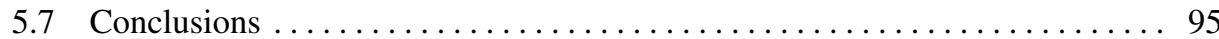

5.8 Appendix: Optimal CMS frequency $\ldots \ldots \ldots \ldots \ldots \ldots \ldots \ldots \ldots \ldots \ldots$

$6 \quad$ Evaluation ............................................... 97

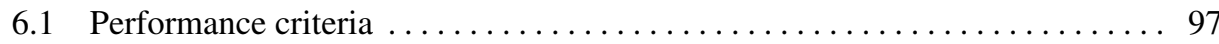

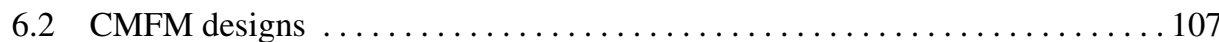

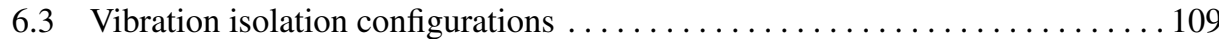

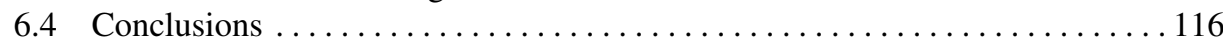

7 Conclusions and Recommendations . . . . . . . . . . . . . . . . . . . . . . . . . . 119

7.1 Conclusions .............................................. 119

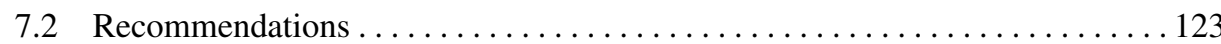

References.................................................. 125

Part II Appendices

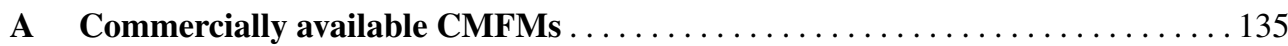

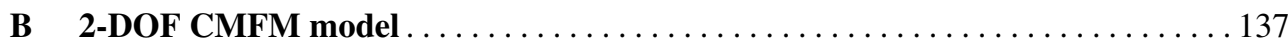

C Patent: CMFM with active vibration isolation control $\ldots \ldots \ldots \ldots \ldots \ldots \ldots 145$

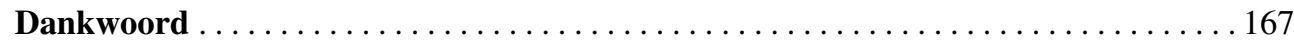




\section{Nomenclature}

\section{Mathematical notation}

$\begin{array}{ll}x & \text { Scalar } \\ \boldsymbol{x} & \text { Vector } \\ \boldsymbol{X} & \text { Matrix } \\ \boldsymbol{\bullet} & \text { First derivative of } \bullet \text { with respect to time } \\ \bullet & \text { Second derivative of } \bullet \text { with respect to time } \\ \hat{\bullet} & \text { Estimate of } \bullet \\ \bullet & \text { Filtered signal } \bullet \\ \bullet(t) & \text { Continuous time signal } \\ \bullet(s) & \text { Laplace transformed variable or continuous system } \\ \bullet(z) & \text { Discrete-time system } \\ \bullet(\omega) & \text { Fourier transformed variable or value of } \bullet \text { at } \omega \\ \bullet T & \text { Transverse of } \bullet \\ \bullet-1 & \text { Inverse of } \bullet \\ \bullet \dagger & \text { Pseudo inverse of } \bullet \\ \bullet \cdot \bullet & \text { Inner product } \\ \bullet \times \bullet & \text { Outer product } \\ \bullet \bullet & \text { Absolute value of } \bullet \\ \mathfrak{R}(\bullet) & \text { Real part of } \bullet \\ \mathfrak{I}(\bullet) & \text { Imaginary part of } \bullet\end{array}$




$\begin{array}{ll}\text { DN } & \text { Diameter Nominal } \\ \text { DOF } & \text { Degree Of Freedom } \\ \text { DUT } & \text { Device Under Test } \\ \text { FB } & \text { Feedback } \\ \text { FEM } & \text { Finite Element Method } \\ \text { FF } & \text { Feed Forward } \\ \text { FRF } & \text { Frequency Response Function } \\ \text { FxLMS } & \text { Filtered reference Least Mean Square } \\ \text { HPF } & \text { High Pass Filter } \\ \text { IIR } & \text { Infinite Impulse Response } \\ \text { LPF } & \text { Low Pass Filter } \\ \text { MIMO } & \text { Multiple Input Multiple Output } \\ \text { NF } & \text { Nominal Flow } \\ \text { PCB } & \text { Printed Circuit Board } \\ \text { PI } & \text { Proportional Integral } \\ \text { PSD } & \text { Power Spectral Density } \\ \text { RMS } & \text { Root Mean Square } \\ \text { ROI } & \text { Region of Interest } \\ \text { SISO } & \text { Single Input Single Output } \\ \text { SVD } & \text { Singular Value Decomposition } \\ \text { VC } & \text { Vibration Criterion } \\ \text { VI } & \text { Vibration Isolation } \\ \text { ZS } & \text { Zero Stability }\end{array}$

\section{Greek symbols}

$\begin{array}{ll}\alpha & \text { Cut-off frequency in feedforward controller (rad/s) } \\ \alpha & \text { Ratio between Coriolis and actuation frequency } \\ \Gamma & \text { First order geometric transfer function } \\ \zeta & \text { Relative damping } \\ \zeta & \text { Trajectory parameter of the tube-window } \\ \zeta_{\mathrm{f}} & \text { Damping ratio of second-order LPF in controller } \\ \zeta_{\mathrm{r}} & \text { Damping ratio of second-order HPF in controller } \\ \theta_{\text {swing }} & \text { Angle of rotation around the swing-axis } \\ \theta_{\text {twist }} & \text { Angle of rotation around the twist-axis } \\ \mu & \text { Adaptation rate } \\ v & \text { Frequency range }(\mathrm{rad} / \mathrm{s}) \\ v & \text { Dynamic viscosity }\left(\mathrm{m}^{2} / \mathrm{s}\right) \\ \rho & \left.\text { Density (kg/m }{ }^{3}\right) \\ \sigma . & \text { Root mean square of signal } \bullet \\ \phi . & \text { Phase of signal } \bullet(\mathrm{rad}) \\ \Delta \phi & \text { Phase difference (rad) } \\ \dot{\Phi}_{\mathrm{m}} & \text { Mass-flow } \\ \Phi . & \text { Power spectral density of signal }\end{array}$




$\begin{array}{ll}\omega_{1} & \text { Suspension mode frequency }(\mathrm{rad} / \mathrm{s}) \\ \omega_{2} & \text { Internal mode frequency }(\mathrm{rad} / \mathrm{s}) \\ \omega_{\mathrm{act}} & \text { Actuation frequency }(\mathrm{rad} / \mathrm{s}) \\ \omega_{\mathrm{ar}} & \text { Anti-resonance frequency }(\mathrm{rad} / \mathrm{s}) \\ \omega_{\mathrm{cor}} & \text { Coriolis frequency }(\mathrm{rad} / \mathrm{s}) \\ \omega_{\mathrm{f}} & \text { Corner frequency of second-order LPF in controller }(\mathrm{rad} / \mathrm{s}) \\ \omega_{i} & \text { Resonance frequency }(\mathrm{rad} / \mathrm{s}) \\ \omega_{\text {ind }} & \text { Induction pole actuator }(\mathrm{rad} / \mathrm{s}) \\ \omega_{\mathrm{r}} & \text { Corner frequency of second-order HPF in controller }(\mathrm{rad} / \mathrm{s}) \\ \omega_{\mathrm{x}} & \text { Centre frequency of bandpass filter }(\mathrm{rad} / \mathrm{s}) \\ \omega_{\mathrm{z}} & \text { High frequency zero }(\mathrm{rad} / \mathrm{s})\end{array}$

\section{Roman symbols}

$\begin{array}{ll}a_{0} & \text { External vibration } \\ a_{1} & \text { Stage vibration } \\ a c t . & \text { Tube actuator } \\ A & \text { Cross sectional area } \\ \boldsymbol{C} & \text { Velocity sensitive matrix (Ns/m) } \\ C & \text { Controller } \\ \mathbb{C} & \text { Set of complex numbers } \\ d & \text { Tube diameter (m) } \\ d_{1} & \text { Suspension damping } \\ d_{2} & \text { Tube damping } \\ \boldsymbol{D} & \text { Damping matrix (Ns/m) } \\ e & \text { Error signal } \\ f_{i} & \text { Resonance frequency (Hz) } \\ F & \text { Bandpass filter in phase demodulation algorithm } \\ F & \text { Filter in adaptive feedforward algorithm } \\ F_{\mathrm{a}} & \text { Actuator force } \\ F_{\text {cor }} & \text { Coriolis force } \\ F_{\mathrm{dis}} & \text { Disturbance force } \\ g & \text { Gain } \\ G & \text { Transfer function } \\ H & \text { Part of controller } \\ \mathrm{i}=\sqrt{-1} & \text { Imaginary unit } \\ \mathrm{j}=\sqrt{-1} & \text { Imaginary unit } \\ J & \text { Quadratic cost function } \\ k_{1} & \text { Suspension stiffness } \\ k_{2} & \text { Tube stiffness } \\ k_{\mathrm{m}} & \text { Motor constant (N/A) } \\ K_{\mathrm{a}} & \text { Proportional gain } \\ K_{\mathrm{V}} & \text { Integral gain } \\ \boldsymbol{K} & \text { Stiffness matrix (N/m) } \\ & \end{array}$




\begin{tabular}{|c|c|}
\hline$L$ & Induction $(\mathrm{mH})$ \\
\hline$L$ & Effective tube length (m) \\
\hline$m_{1}$ & Stage mass \\
\hline$m_{2}$ & Tube mass \\
\hline$M_{\text {act }}$ & Actuation moment \\
\hline$M$ & Mass matrix (kg) \\
\hline$n$. & Noise signal $\bullet$ \\
\hline$n$. & Number of signals $\bullet$ \\
\hline$N$ & Dynamic stiffness matrix $(\mathrm{N} / \mathrm{m})$ \\
\hline$N$ & Bandpass filter \\
\hline$P$ & Plant transfer function \\
\hline$P$ & Pressure $\left(\mathrm{N} / \mathrm{m}^{2}\right)$ \\
\hline$\Delta P$ & Pressure difference $\left(\mathrm{N} / \mathrm{m}^{2}\right)$ \\
\hline$q$ & Degree of freedom \\
\hline$Q$ & Quality factor \\
\hline$\vec{r}$ & Half pitch of the tube displacement sensors \\
\hline $\boldsymbol{R}$ & Transformation matrix \\
\hline$R$ & Resistance $(\Omega)$ \\
\hline $\mathbb{R}$ & Set of real numbers \\
\hline$s$ & Laplace s-transform \\
\hline$s_{\bullet}$ & Tube displacement sensor $\bullet$ \\
\hline$S$ & Sensitivity for flow \\
\hline$S$ & Sensitivity transfer function \\
\hline$T$ & Transmissibility \\
\hline$T_{\mathrm{s}}$ & Sample time (s) \\
\hline$u$ & Fluid velocity $(\mathrm{m} / \mathrm{s})$ \\
\hline$u$ & Input vector \\
\hline$v_{i}$ & Eigen vector \\
\hline $\boldsymbol{V}$ & Modal matrix $\boldsymbol{V}=\left[\boldsymbol{v}_{1}, \boldsymbol{v}_{2}, \cdots, \boldsymbol{v}_{n}\right]$ \\
\hline$w$ & Weights vector \\
\hline$x$ & Filtered reference signal \\
\hline$x$ & Orthogonal coordinates $\boldsymbol{x}=\left(x, y, z, \theta_{x}, \theta_{y}, \theta_{z}\right)^{T}$ \\
\hline$x$ & State vector \\
\hline$y$ & Output vector \\
\hline$y_{\text {act }}$ & Actuation displacement \\
\hline$y_{\text {cor }}$ & Coriolis displacement \\
\hline$z$ & Modal coordinate \\
\hline
\end{tabular}




\section{Part I}

Thesis 



\section{Chapter 1 \\ Introduction}

Vibration isolation for Coriolis Mass-Flow Meters (CMFM) is the main topic of this thesis. This chapter starts with a discussion on the advantages of using a CMFM for a mass-flow measurement. Thereafter, the current state of the art is presented and the necessity for vibration isolation is explained. The research objective and approach of this thesis are discussed next, followed by the scientific contributions of this research and the thesis outline.

\subsection{Background}

Flow measurement is a technique used in many processes, requiring a determinable amount of a material transported from one point to another [3, 21]. It can be used for quantifying an amount of material or controlling a specific flow rate. Application examples are: (i) flow-rate measurement of oil or gas consumption, (ii) batch filling of food and beverage containers and (iii) additive dosage (Fig. 1.2) of flavours or fragrances to a main flow [9]. A measure of the transported mass can be achieved by integration of the flow rate over time. The measurement of mass-flow rate $(\mathrm{kg} / \mathrm{s})$ has certain advantages over volume flow rate $\left(\mathrm{m}^{3} / \mathrm{s}\right)$ measurements, namely pressure, temperature, and density of the fluid do not have to be considered to obtain the transported amount of a fluid.

A Coriolis Mass-Flow Meter (CMFM) is based on measuring the force or induced motion, as a measure of the mass-flow, when subjecting the fluid stream to a Coriolis acceleration. The acceleration is named after Gustave Coriolis [15], see Gerkema and Gostiaux [22] for a brief history of the Coriolis force. This principle can be used for true mass-flow measurements, in which the measured parameter is directly related to mass-flow, independently of the fluid properties. Market research studies show an increasing acceptance of the CMFMs by various industries, making it one of the fastest growing flow meter markets [20, 78].

A typical tube design of a CMFM is depicted in Fig. 1.1(a) to explain the working principle. The design consists of a window-shaped tube. The tube is actuated to oscillate in resonance with a low amplitude around the $\theta_{\text {twist }}$-axis. A fluid flow in the vibrating tube induces a Coriolis force on the tube, proportional to the mass-flow $\dot{\Phi}_{\mathrm{m}}$ : 


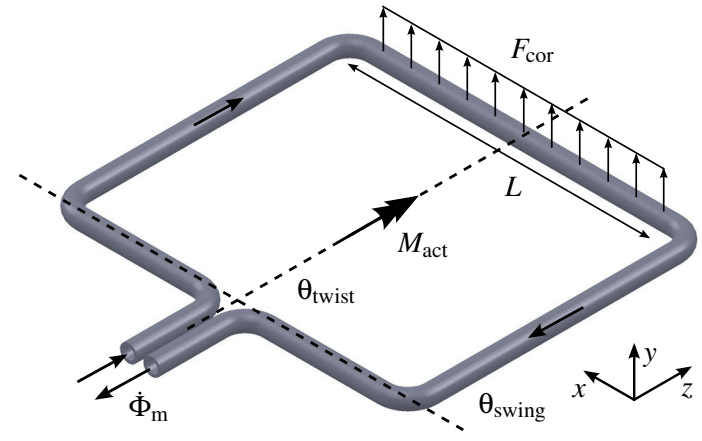

(a)

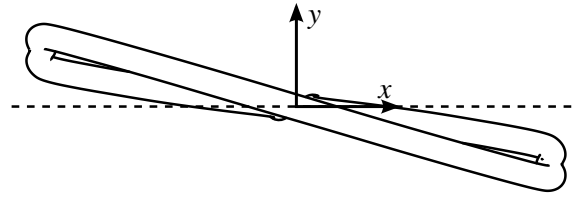

(b) Actuation mode shape - without flow

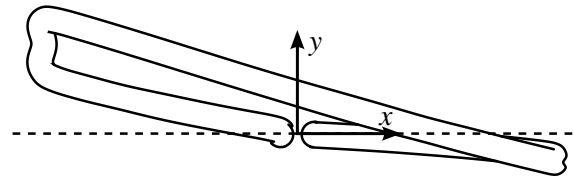

(c) Actuation mode shape - with flow

Fig. 1.1 Explanation of the Coriolis principle, using a window-shaped fluid-conveying tube. The tube is actuated to resonate around the $\theta_{\text {twist }}$-axis. Due to the Coriolis effect the tube also oscillates around the $\theta_{\text {swing }}$ axis, proportional to the mass-flow $\dot{\Phi}_{\mathrm{m}}$, resulting in an altered mode-shape.

$$
F_{\text {cor }}=-2 L \cdot \dot{\theta}_{\text {twist }} \times \dot{\Phi}_{\mathrm{m}}
$$

where, $L$ is the effective length of the tube and $\dot{\theta}_{\text {twist }}$ the angular velocity. Due to the geometry and dynamic properties of the tube, this force results in a rotation around the $\theta_{\text {swing }}$-axis. The position of the axis is dependent on the tube-fixation, dimensions and fluid density. The force is $90^{\circ}$ out-of-phase with the actuation displacement, and thus the mode shape of the actuation mode is affected, see Fig. 1.1(b) and 1.1(c). The effect of a mass-flow is in the order of nanometres for micrometre actuation displacements. Measuring the tube motion at several locations, allows measuring the rotations around the twist- and swing-axis of which the ratio is directly proportional to the mass-flow. The actuation mode shape without flow is depicted in the bottom right corner of the odd pages and on the even pages on the bottom left for the mode shape with flow. An extended explanation of the measurement principle follows in Chapter 2 and 6

A CMFM is a mechatronic system used to measure the mass-flow of liquids or gases with a high accuracy, range-ability and repeatability and without prior knowledge of the fluid. The density can also be determined, from its effect on the resonance frequency, so also the volume flow-rate can be calculated indirectly.

\subsection{Current state of the art}

Many CMFMs for various flow ranges are available nowadays. An example of a low-flow CMFM is given in Fig. 1.3. In Appendix A an overview and in Table.1.1 a specification summary is given of commercially available CMFMs. Since the 'Coriolis mass flowmeter' was mentioned first by White [80] in 1958, the design improved significantly. Recently Wang and Baker [78] presented the developments over the past 20 years, showing the recent advances 


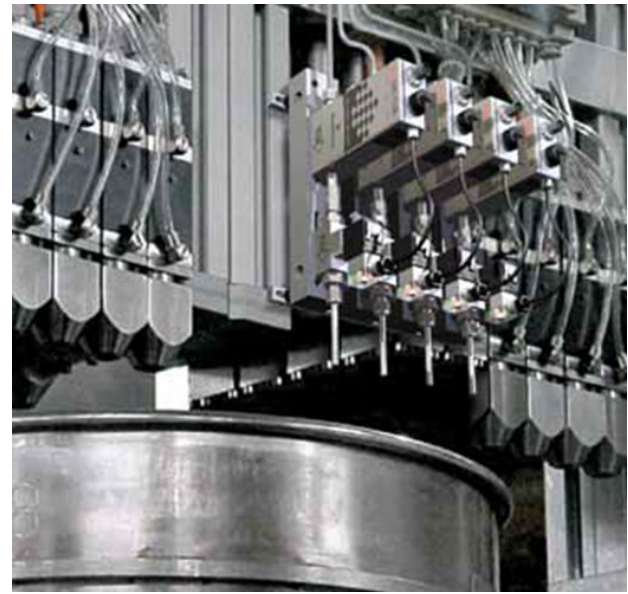

Fig. 1.2 Example of using a CMFM for additive dosage [8].

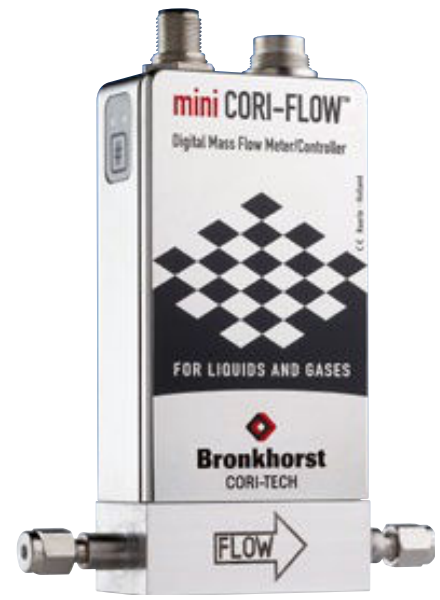

Fig. 1.3 Bronkhorst mini Cori-Flow M13 with a measurement range of $1-1000 \mathrm{~g} / \mathrm{h}$ [8].

Table 1.1 Specification summary of commercially available Coriolis Mass-Flow Meters [1, 78].

\begin{tabular}{ll}
\hline Measured variables & Mass-flow, volume-flow, density, viscosity, temperature, concentration \\
Nominal flow range & From $1 \mathrm{~g} / \mathrm{h}$ up to $4100 \mathrm{t} / \mathrm{h}$ \\
Fluids & Liquids, slurries, gases, liquefied gases \\
Tube diameter & From less than $0.25 \mathrm{~mm}$ to $205 \mathrm{~mm}$ \\
Operating pressure & Up to $413 \mathrm{bar}$ \\
Operating temperature & From -240 up to $427{ }^{\circ} \mathrm{C}$ \\
Materials & Stainless steel $316 / 316 \mathrm{~L}$, Alloy C-22, Titanium, Tantalum and others \\
Accuracy & Typically $0.05 \%$ to $0.5 \%$ for liquids and $0.35 \%$ to $0.75 \%$ for gases \\
Zero stability & From $0.002 \%$ to $0.1 \%$ of the nominal flow rate \\
Repeatability & Typically half the accuracy specification \\
Range-ability & $1000: 1$ \\
\hline
\end{tabular}

in fundamental understanding and technology development. They also outlined several open questions.

For the understanding of the fluid-structure interaction, there are a large number of publications. Païdoussis [51] presented various fundamental theories and also the governing equations of a CMFM. Sultan and Hemp [61] modelled a CMFM to predict the phase shifts due to flow. Later, Hemp [28] presented a weight vector approach to calculate the sensitivity for flow. Those, and even more, models [25, 35, 37, 54] resulted in CMFMs with better and better specifications to be an 'ideal' flow meter.

However, besides measuring the mass-flow, there are various types of disturbances affecting the measurement value. Anklin et al. [1] mentioned several factors: the effect of temperature and flow profiles on the sensitivity and measurement value, external vibrations and flow pulsations. More factors are investigated by Enz et al. [18]: Flow pulsations, asymmetrical actuator and detector positions and structural non-uniformities, and more recently also by Kazahaya [35]: uneven flow rates in two flow tubes, vibration effects, temperature effects

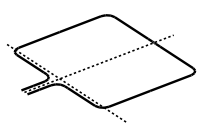


and the inner pressure effects. Further Bobovnik et al. [5, 6] studied the effect of disturbed velocity profiles due to the shape of the fluid supply tubes and other influencing factors like two-phase or even three-phase flow effects were studied by Henry et al. [29]. Wang and Baker [78] discussed recently also the different signal processing and control techniques. Recent developments in digital hardware bring improved performance, which actually affects the measurement value. Coriolis forces and tube imperfections, like asymmetric distribution of the mass, damping and stiffness properties, both result in static phase shifts [19, 62, 68]. No distinction can be made yet, and thereby those imperfections affect the zero measurement value. Furthermore, external stresses and vibrations may affect the measurement value [12, 14]. External vibrations at the meter's drive frequency produce a measurement error, regardless of the flow measurement algorithm [13].

Before starting this project, experimental results showed an increased sensitivity for external vibrations, especially when scaling down the nominal flow range $(<1 \mathrm{~kg} / \mathrm{h})$. Therefore, the focus in this thesis will be on developing a quantitative model and obtaining vibration isolation for a CMFM.

\subsection{Research objective and approach}

The continuous effort for improving the performance of CMFMs is hampered by the effect of external vibrations on the measurement value, especially when scaling down the flow range. The effect of vibrations is known, but so far the effect has not been clearly quantified, which hinders the implementation of effective measures. Therefore, the research objective of this thesis is formulated as:

Investigate the effect of external vibrations on the performance of a Coriolis Mass-Flow Meter by quantitative modelling of the influence of external vibrations. Based on these insights, develop methods to reduce the effect of vibrations significantly (factor 100 or $40 \mathrm{~dB}$ ) without changing the performance and validate these conceptual solutions experimentally.

The approach to achieve the research objective starts with developing a flexible multibody model of a CMFM, using the MATLAB package SPACAR [31, 33]. A flexible fluidconveying tube-element [47] is used to determine the mode shapes, including the mass-flow induced effects, due to the Coriolis force. The model is also able to quantify the effect of vibrations on the tube motion. Based on these results and knowledge of the algorithms that compute the fluid flow, the sensitivity of the measurement value for flow and external vibrations is quantified. This result is the basis for the investigation of various passive and active vibration isolation concepts, which reduce the effect of external vibrations but leave the sensitivity for flow unaffected.

To reduce the influence of external vibrations, passive or active vibration isolation can be used. Passive isolation consists of one or multiple stages of mass-spring-damper systems between an excitation source and an influenced receiver (e.g. the floor and the casing of a

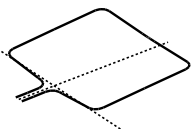


machine) [53]. The parameters are adjusted to achieve high-frequency attenuation, which is suitable for many applications. However, the performance of passive isolation applied to a CMFM is limited due to a minimal suspension frequency, which is caused by the parasitic stiffness of the fluid-connecting-tubes and the maximum allowable gravitational sagging of the stage. Large deformations in the fluid-connecting-tubes would result in high internal stresses. An alternative approach is to apply active vibration isolation control to a flexibly suspended single-tube configuration. This is an application of active hard mount isolation for precision equipment [64,76].

Active vibration isolation control is used, in Chapter 3-5, to achieve attenuation in a limited frequency band, while maintaining the positive effects of the passive support stiffness. This is sufficient for minimisation of the influence of external vibrations on the mass-flow measurement. Therefore, different control algorithms based on feedback and feedforward strategies are compared. The control algorithm for feedback is a combination of known feedback strategies for vibration isolation [34, 52, 77]. The novelty is the smart combination of those algorithms -acceleration feedback, velocity feedback, internal mode damping, cascade control and loop shaping- tuned, modified and applied to the specific application of CMFMs.

The feedforward approach is based on a novel strategy, using Infinite Impulse Response filters with fixed poles and adaptive weights. The weights only depend on the physical parameters - stiffness, damping and actuator dynamics - between the floor and the suspended stage. The amount of weights is much lower than for conventional adaptive feedforward approaches [36, 38]. This strategy is developed together with Beijen et al. [4], who presented the general approach to use it as feedfoward control for vibration isolation of precision machines. The requirement that the flow measurement should not be affected, is used in this work to make some dedicated modifications to the strategy to make it even more effective for a CMFM.

The concepts are elaborated in two functional models to validate the modelling and isolation concepts. Based on these results it is discussed, how vibration isolation can be integrated in CMFMs in the future.

\subsection{Contributions}

The scientific output of this research is presented at various international conferences and is published in various journals. The research also resulted in several patent applications. An overview of all scientific output:

\section{Journal articles:}

- L. van de Ridder, W. B. J. Hakvoort, D. M. Brouwer, J. van Dijk, J. C. Lötters, and A. de Boer. Coriolis mass-flow meter with integrated multi-dof active vibration isolation. 2015. Submitted to Mechatronics (Elsevier),

- L. van de Ridder, W. B. J. Hakvoort, J. van Dijk, J. C. Lötters, and A. de Boer. Vibration isolation by an actively compliantly mounted sensor applied to a coriolis mass-flow meter. 2015. Submitted to Dynamic Systems, Measurement and Control (ASME), 
- L. van de Ridder, M. A. Beijen, W. B. J. Hakvoort, J. van Dijk, J. C. Lötters, and A. de Boer. Active vibration isolation feedback control for coriolis mass-flow meters. Control Engineering Practice, 33C:76-83, 2014. URL http://dx.doi.org/10.1016/ j.conengprac.2014.09.007,

- L. van de Ridder, W. B. J. Hakvoort, J. van Dijk, J. C. Lötters, and A. de Boer. Quantification of the influence of external vibrations on the measurement error of a coriolis massflow meter. Flow Measurement and Instrumentation, 40C:39-49, 2014. URL http:// dx.doi.org/10.1016/j.flowmeasinst.2014.08.005.

\section{Conference Proceedings:}

- L. van de Ridder, W. B. J. Hakvoort, and J. van Dijk. Active vibration isolation control: comparison of feedback and feedforward control strategies applied to coriolis mass-flow meters. In ACC 2015 The American Control Conference, Chicago, IL, USA, 1-3 July 2015. URL http://doc.utwente.nl/95710/,

- K. Staman, L. van De Ridder, W. B. J. Hakvoort, D. M. Brouwer, and J. van Dijk. A multi-dof active vibration isolation setup for a coriolis mass-flow meter. In 29th Annual Meeting of the American Society for Precision Engineering, Boston, Massachusetts USA, 9-14 November 2014. URL http://doc . utwente.nl/93719/,

- J. Groenesteijn, L. van de Ridder, J. C. Lötters, and R. J. Wiegerink. Modelling of a micro coriolis mass flow sensor for sensitivity improvements. In Proceedings of the Thirteenth IEEE Sensors Conference, IEEE Sensors 2014, Valencia, Spain, pages 954 957, Valencia, Spain, 2-5 November 2014. IEEE Service Center. URL http://doc. utwente.nl/92825/,

- L. van de Ridder, W. B. J. Hakvoort, and J. van Dijk. Vibration isolation by compliant sensor mounting applied to a coriolis mass-flow meter. In ASME 2014 12th biennial conference on engineering systems design and analysis (ESDA2014), Copenhagen, Denmark, 25-27 June 2014. URL http://doc. utwente.nl/91292/,

- L. van de Ridder, W. B. J. Hakvoort, J. van Dijk, J. C. Lötters, and A. de Boer. Quantitative estimation of the influence of external vibrations on the measurement error of a coriolis mass-flow meter. In Z. Dimitrovová, J.R. de Almeida, and R. Gonçalves, editors, 11th International Conference on Vibration Problems (ICOVP-2013), Lisbon, Portugal, 9-12 September 2013. URL http://doc. utwente.nl/89168/,

- L. van de Ridder, W. B. J. Hakvoort, J. van Dijk, and J. C. Lötters. Influence of external damping on phase difference measurement of a coriolis mass-flow meter. In EUROMECH Colloquium 524, Enschede, 27-29 February 2012. URL http://doc. utwente.nl/89167/.

\section{Patents:}

- J. C. Lötters, L. van de Ridder, W. D. Kruijswijk, W. B. J. Hakvoort, and M. R. Katerberg. Coriolis flow meter with active vibration isolation, Patent pending, 25 November 2013,

- J. C. Lötters, L. van de Ridder, W. D. Kruijswijk, W. B. J. Hakvoort, and M. R. Katerberg. Coriolis flow meter with passive vibration isolation, Patent pending, 24 March 2014. 


\subsection{Outline}

The main part of this thesis is a collection of journal articles, appearing as reprints in Chapters 2 to 5 The journal articles are self-contained and can be read independently. Note, that pieces are repeated in multiple chapters. The articles are supplemented by a comparison, discussion and conclusion in Chapters 6 and 7. The content of all the chapters is described below:

Chapter 2 describes a flexible 3D multi-body model of a CMFM. This model is used to investigate and quantify the influence of external vibrations. Experimental results confirm that the transfer function from external vibrations to the Coriolis displacement can be used to estimate the influence.

In Chapter 3 an active vibration isolation feedback strategy is analysed to reduce the transfer function from external vibrations to the Coriolis displacement. The casing of an existing CMFM is passively suspended and active means are added to apply acceleration feedback.

Chapter 4 presents an on-scale functional model of a CMFM with integrated active vibration isolation control. In addition, the feedback strategy is compared to an adaptive feedforward strategy, whereby the weights are updated to minimise the measurement error.

In Chapter 5 a second strategy to apply vibration isolation to a CMFM is analysed. Instead of an absolute measurement of the tube displacements, a relative displacement is introduced by a flexible mounting of the displacement sensors. This configuration also reduces the transfer function from vibrations to the Coriolis displacement. A functional model is presented, validating that this concept can realise vibration isolation.

Chapter 6 starts with a reduced model of a CMFM to show the effect of scaling down the flow range on the vibration sensitivity. Further, an overview is given of the analysed configurations to apply vibration isolation to a CMFM.

Conclusions of this research are presented in Chapter 7 followed by recommendations for further research.

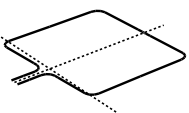




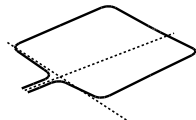




\title{
Chapter 2 \\ Quantification of the influence of external vibrations
}

\begin{abstract}
In this paper the influence of external vibrations on the measurement value of a Coriolis Mass-Flow Meter (CMFM) for low flows is investigated and quantified. Model results are compared with experimental results to improve the knowledge on how external vibrations affect the mass-flow measurement value. A flexible multi-body model is built and the working principle of a CMFM is explained. Some special properties of the model are evaluated to get insight in the dynamic behaviour of the CMFM. Using the model, the transfer functions between external vibrations (e.g. floor vibrations) and the flow error are derived. The external vibrations are characterised with a PSD. Integrating the squared transfer function times the PSD over the whole frequency range results in an RMS flow error estimate. In an experiment predefined vibrations are applied on the casing of the CMFM and the error is determined. The experimental results shows that the transfer functions and the estimated measurement error correspond with the model results. The agreement between model and measurements implies that the influence of external vibrations on the measurement is fully understood. This result can be applied in two ways; firstly that the influence of any external vibration spectrum on the flow error can be estimated and secondly that the performance of different CMFM designs can be compared and optimised by shaping their respective transfer functions.
\end{abstract}

This chapter is reprinted from: L. van de Ridder, W. B. J. Hakvoort, J. van Dijk, J. C. Lötters, and A. de Boer. Quantification of the influence of external vibrations on the measurement error of a coriolis mass-flow meter. Flow Measurement and Instrumentation, 40C: 39-49, 2014. URL http://dx.doi.org/10.1016/j.flowmeasinst.2014.08.005, with permission from Elsevier.

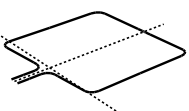




\subsection{Introduction}

A Coriolis Mass-Flow Meter (CMFM) is an active device based on the Coriolis force principle for direct mass-flow measurements with a high accuracy, range-ability and repeatability [1]. The working principle of a CMFM is as follows: a fluid conveying tube is actuated to oscillate with a low amplitude at a resonance frequency in order to minimise the amount of supplied energy. A fluid flow in the vibrating tube induces Coriolis forces, proportional to the mass-flow, which affect the tube motion and change the mode shape. Measuring the tube displacement, such that the change of its mode shape is determined, allows calculating the mass-flow.

Besides the sensitivity for a mass-flow, there are many factors influencing the measurement value. Anklin et al. [1] mentioned several factors: the effect of temperature and flow profiles on the sensitivity and measurement value, external vibrations and flow pulsations. More factors are investigated by Enz et al. [18]: Flow pulsations, asymmetrical actuator and detector positions and structural non-uniformities. And more recent also by Kazahaya [35]: uneven flow rates in two flow tubes, vibration effects, temperature effects and the inner pressure effects. Further Bobovnik et al. [5] studied the effect of disturbed velocity profiles due to installation effects and other influencing factors like two-phase or even three-phase flow effects were studied by Henry et al. [29]

In our research we focus mainly on the effect of floor/mechanical/external vibrations. These vibrations create additional components in the CMFM sensor signals [14], those additional components can introduce a measurement error. The effect of mechanical vibrations on the sensor response of a CMFM is also studied by Cheesewright [12, 13]. The analytical study showed that external vibrations at the meter's drive frequency produces a measurement error, regardless of the flow measurement algorithm. There is no attempt made to quantify the error in any particular meter, since such an error depends on dimensions, type of actuators and sensors and the used flow measurement algorithm.

A solution to reduce the influence of external vibrations is to apply a robust balancing system. (e.g. a twin tube configuration) [1, 35]. There are many types of CMFMs available, whereby the size depends on the flow range. One category is the CMFM for low flows [44]. For low flows, the Coriolis force induced motion is relative small compared to external vibrations induced motions, thus CMFM's designed to be sensitive to low flows, are rather sensitive to external vibrations. Applying a twin tube configuration is not an option, because some structural non-uniformities [18] can lead to large differences between the two tubes, due to their small dimensions. This has an negative impact on the measurement sensitivity of the instrument and reduces the decoupling of external vibrations to the internal measurement system.

A quantitative model of the influence of external vibrations is not yet available. In this study the effect of external vibrations on the measurement error is quantified using an experimentally validated model. The results presented in this study are an extension of previous work [69]. First, a model of a CMFM is derived, using the multi-body package SPACAR [31] resulting in a linear state space representation [32]. In the modelling, a tube-element [47] is used to model the inertial interaction between flow and the tube dynamics. Secondly, the model is extended to be able to predict the influence of external vibrations, with the eventual

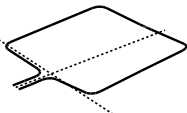


goal to find and test designs that reduce the influence of external vibrations on an erroneous mass-flow reading.

\subsection{Modelling method}

In this section, the Finite Element Method (FEM) model is explained. Subsequently, the system equations are derived and the inputs and outputs are defined to derive the input-output relations. This results in a state space representation of a CMFM in the final subsection.

\subsubsection{Coriolis Mass-Flow Meter}

For this research a functional model of the patented design [44, 45] (see Fig. 2.1) is used. First, a FEM model is derived, using the multi-body package SPACAR [31]. The graphical representation of the model is shown in Fig. 2.2. The model consists of a tube-window, conveying the fluid flow, which is actuated by two actuators $a c t_{1}$ and $a c t_{2}$. The displacements of the flexible tube-window are measured by two displacements sensors $s_{1}$ and $s_{2}$. On the casing a vector $\boldsymbol{a}_{0}$, representing the external vibrations and consisting of three translation and three rotational movements, is imposed. The model is made out of multi-body beam, truss and tube elements. The beam elements are used to model the rigid casing and the truss elements to measure relative displacements and to apply a force on the tube-window. Further, a tube-element [47] is used to model the inertial interaction between flow and the tube dynamics.

\subsubsection{System equations}

The linearised system equations of the FEM model, with $n$ degrees of freedom of tube deformations $\boldsymbol{q}$ and the imposed casing movements (rheonomic degrees of freedom: $\boldsymbol{x}_{0}, \boldsymbol{v}_{0}=$ $\dot{\boldsymbol{x}}_{0}, \boldsymbol{a}_{0}=\ddot{\boldsymbol{x}}_{0}$ ), can be written as [32]:

$$
\left[\begin{array}{ll}
\boldsymbol{M}_{11} & \boldsymbol{M}_{12} \\
\boldsymbol{M}_{21} & \boldsymbol{M}_{22}
\end{array}\right]\left[\begin{array}{c}
\ddot{\boldsymbol{q}} \\
\boldsymbol{a}_{0}
\end{array}\right]+\left[\boldsymbol{C}\left(\dot{\Phi}_{\mathrm{m}}\right)+\boldsymbol{D}\right]\left[\begin{array}{c}
\dot{\boldsymbol{q}} \\
\boldsymbol{v}_{0}
\end{array}\right]+\left[\boldsymbol{K}+\boldsymbol{N}\left(\dot{\Phi}_{\mathrm{m}}^{2}\right)\right]\left[\begin{array}{c}
\boldsymbol{q} \\
\boldsymbol{x}_{0}
\end{array}\right]=\left[\begin{array}{c}
\boldsymbol{f} \\
\boldsymbol{F}_{0}
\end{array}\right]
$$

The other terms are the mass matrix $\boldsymbol{M}$, stiffness matrix $\boldsymbol{K}$, damping matrix $\boldsymbol{D}$, the velocity sensitive matrix $C$, the dynamic stiffness matrix $N$, the actuation input vector $f$ and the reaction force $\boldsymbol{F}_{0}$. The matrices $\boldsymbol{C}$ and $\boldsymbol{N}$ depend linear and quadratic on the mass-flow $\dot{\Phi}_{\mathrm{m}}$ respectively, and are representing the forces induced by respectively the Coriolis and centrifugal acceleration of the flow. The matrices $\boldsymbol{C}, \boldsymbol{D}, \boldsymbol{K}$ and $\boldsymbol{N}$ can be divided in same parts as the mass matrix $\boldsymbol{M}$. Using the multi-body package SPACAR [31] the system matrices 


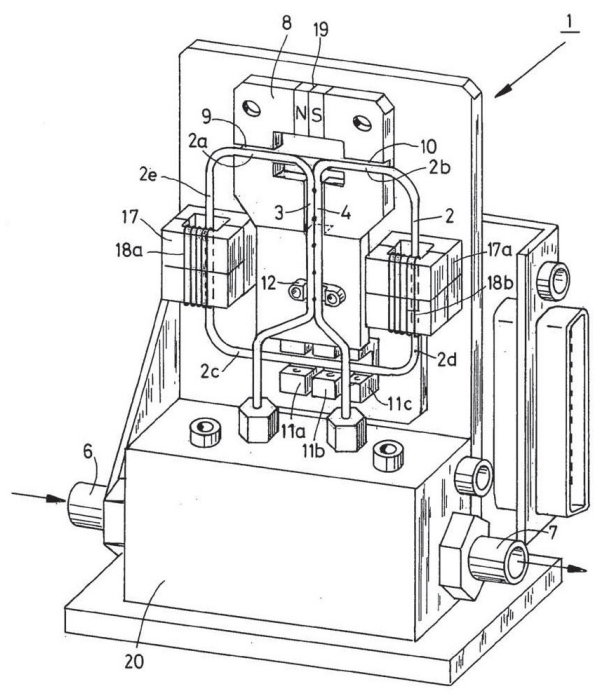

Fig. 2.1 Coriolis Mass-Flow Meter, used as a reference instrument in this study. Details on the patented design are given in [44, 45]. The instrument is connected to a pipeline; a fluid flow enters the instrument (6), flows trough the tubewindow (2) and exits the instrument (7). The flexible tube-window (2) is actuated in resonance by an Lorentz actuator (8) and the displacements are measured by optical displacements sensors (11abc) [46].

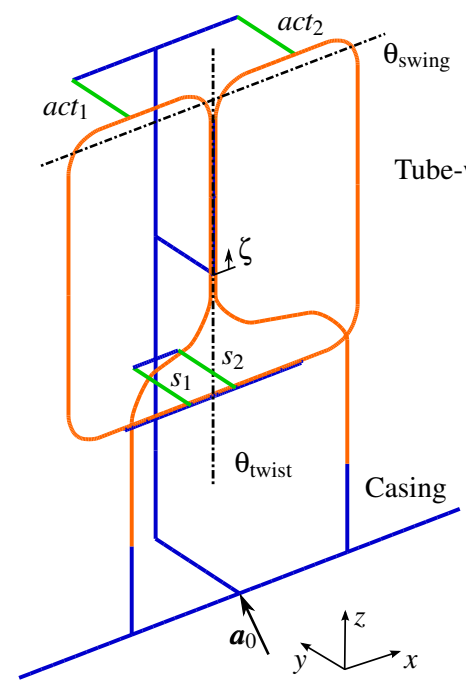

Fig. 2.2 CMFM multi-body model, the flexible tube-window is actuated by two Lorentz actuators $a c t_{1}$ and $a c t_{2}$. The trajectory of the curved tube-window is parametrised by $\zeta$, starting at the fixation point of the tube-window to the casing. The displacement are measured by two displacements sensors $s_{1}$ and $s_{2}$. On the casing a vector $\boldsymbol{a}_{0}$ with floor movements is imposed.

with respect to the element deformations and the imposed floor movements of the model are derived.

The matrices $\boldsymbol{D}_{12}, \boldsymbol{K}_{12}$ and their transposed matrices appears to be zero, due to the choice of element deformations as degrees of freedom. (E.g. $\boldsymbol{K}_{12}=\mathbf{0}$, because there is no coupling between the location of the casing $\boldsymbol{x}_{0}$ and the internal deformations $\boldsymbol{q}$.)

The casing motion is prescribed and thus the only dynamic degrees of freedom are the tube deformations, for which the equations of motion are derived from the top row of Eq.2.1

$$
\boldsymbol{M}_{11} \ddot{\boldsymbol{q}}=\boldsymbol{f}+\boldsymbol{f}_{\mathrm{dis}}-\boldsymbol{C}_{11} \dot{\boldsymbol{q}}-\boldsymbol{D}_{11} \dot{\boldsymbol{q}}-\boldsymbol{K}_{11} \boldsymbol{q}-\boldsymbol{N}_{11} \boldsymbol{q}
$$

including a external disturbance force, consisting of imposed external accelerations:

$$
f_{\text {dis }}=-M_{12} a_{0}
$$

whereby $\boldsymbol{C}_{12} \boldsymbol{v}_{0}$ and $\boldsymbol{N}_{12} \boldsymbol{x}_{0}$ are omitted, because their magnitude is orders lower then $\boldsymbol{M}_{12} \boldsymbol{a}_{0}$. The vector of imposed external accelerations, three translations and three rotations, is equal to: $\boldsymbol{a}_{0}=\left\{\begin{array}{llllll}a_{x} & a_{y} & a_{z} & \alpha_{\mathrm{R}_{x}} & \alpha_{\mathrm{R}_{y}} & \alpha_{\mathrm{R}_{z}}\end{array}\right\}^{T}$

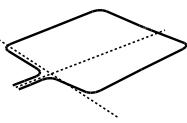


To gain more insight in the model, the degrees of freedom are reduced by applying a modal reduction method. For the modal reduction, the eigenvalue problem $\left(\boldsymbol{K}_{11}+\boldsymbol{N}_{11}-\omega_{i}^{2} \boldsymbol{M}_{11}\right) \boldsymbol{v}_{i}=$ 0 is solved, which results in natural frequencies $\omega_{i}$ and the corresponding eigenvector $\boldsymbol{v}_{i}$, the mode shape. The equations of motion are rewritten in the modal coordinates, defined as: $\boldsymbol{q}=\boldsymbol{V} \boldsymbol{z}$, where $\boldsymbol{V}=\left[\boldsymbol{v}_{1}, \boldsymbol{v}_{2}, \ldots, \boldsymbol{v}_{n}\right]$ is a matrix, normalised such that $\boldsymbol{V}^{T} \boldsymbol{M}_{11} \boldsymbol{V}=\boldsymbol{I}$, of the first $n$ mode shapes and $\boldsymbol{z}$ is the vector of modal amplitudes. Eq.2.2 can now be written as:

$$
\ddot{z}+\boldsymbol{V}^{T} \boldsymbol{C}_{11}\left(\dot{\Phi}_{\mathrm{m}}\right) \boldsymbol{V} \dot{\boldsymbol{z}}+\boldsymbol{V}^{T} \boldsymbol{D}_{11} \boldsymbol{V} \dot{\boldsymbol{z}}+\boldsymbol{V}^{T} \boldsymbol{K}_{11} \boldsymbol{V} \boldsymbol{z}+\boldsymbol{V}^{T} \boldsymbol{N}_{11}\left(\dot{\Phi}_{\mathrm{m}}^{2}\right) \boldsymbol{V} \boldsymbol{z}=\boldsymbol{V}^{T} \boldsymbol{f}+\boldsymbol{V}^{T} \boldsymbol{f}_{\mathrm{dis}}
$$

The reaction forces on the floor can be derived from the lower row of Eq. 2.1

$$
\boldsymbol{F}_{0}=\boldsymbol{M}_{21} \ddot{\boldsymbol{q}}+\boldsymbol{C}_{21} \dot{\boldsymbol{q}}+\boldsymbol{N}_{21} \boldsymbol{q}+\boldsymbol{M}_{22} \boldsymbol{a}_{0}+\left(\boldsymbol{C}_{22}+\boldsymbol{D}_{22}\right) \boldsymbol{v}_{0}+\left(\boldsymbol{K}_{22}+\boldsymbol{N}_{22}\right) \boldsymbol{x}_{0}
$$

\subsubsection{Actuation}

The flexible tube-window is actuated to have an oscillation around the $\theta_{\text {twist }}$-axis (see Fig. 2.2), therefore in the model a moment is applied by two forces between the tube and the casing. In model terms the actuator input is equal to:

$$
\boldsymbol{f}=\frac{1}{r_{\mathrm{M}}}\left(\boldsymbol{\Gamma}_{a c t_{1}}-\boldsymbol{\Gamma}_{a c t_{2}}\right) M_{\mathrm{act}}
$$

where $\boldsymbol{\Gamma}_{a c t_{1}}$ and $\boldsymbol{\Gamma}_{a c t_{2}}$ are vectors with the elongation of the actuator element with respect to the coordinates $\boldsymbol{q}$ of the model, $r_{\mathrm{M}}$ the distance between the two actuator elements and $M_{\text {act }}$ the actuator moment input.

\subsubsection{Sensing}

The movement of the tube-window is measured by two sensors, $s_{1}$ and $s_{2}$. In model terms the sensor displacements are equal to:

$$
s_{i}=\Gamma_{s_{i}} \boldsymbol{q}=\Gamma_{s_{i}} \boldsymbol{V z}
$$

where $\Gamma_{s_{i}}$ is a vector with the elongation of the $i^{\text {th }}$ sensor element with respect to the coordinates $\boldsymbol{q}$ of the model. 


\subsubsection{State Space description}

Combining the equations of the previous sections, a state space representation of the CMFM with a state vector $\boldsymbol{x}=[\boldsymbol{z} \dot{\boldsymbol{z}}]^{T}$, input vector $\boldsymbol{u}=\left[\begin{array}{ll}M_{\mathrm{act}} \boldsymbol{a}_{0}\end{array}\right]^{T}$ and output vector $\boldsymbol{y}=\left[\begin{array}{ll}s_{1} & s_{2}\end{array}\right]^{T}$ is derived:

$$
\begin{aligned}
\dot{\boldsymbol{x}}= & {\left[\begin{array}{cc}
\mathbf{0} & \boldsymbol{I} \\
-\boldsymbol{V}^{T}\left(\boldsymbol{K}_{11}+\boldsymbol{N}_{11}\left(\dot{\Phi}_{\mathrm{m}}^{2}\right)\right) \boldsymbol{V}-\boldsymbol{V}^{T}\left(\boldsymbol{C}_{11}\left(\dot{\Phi}_{\mathrm{m}}\right)+\boldsymbol{D}_{11}\right) \boldsymbol{V}
\end{array}\right] \boldsymbol{x}+} \\
& {\left[\begin{array}{cc}
\mathbf{0} & \mathbf{0} \\
\boldsymbol{V}^{T} \frac{1}{r_{\mathrm{M}}}\left(\boldsymbol{\Gamma}_{a c t_{1}}-\boldsymbol{\Gamma}_{a c t_{2}}\right)-\boldsymbol{V}^{T} \boldsymbol{M}_{12}
\end{array}\right] \boldsymbol{u} } \\
\boldsymbol{y}= & {\left[\begin{array}{ll}
\boldsymbol{\Gamma}_{s_{1}} \boldsymbol{V} & 0 \\
\boldsymbol{\Gamma}_{s_{2}} \boldsymbol{V} & 0
\end{array}\right] \boldsymbol{x}+[\mathbf{0}] \boldsymbol{u} }
\end{aligned}
$$

This state space model can be used to investigate the tube-window displacements as a result of an actuation moment, mass-flow and external vibrations.

\subsection{Model evaluation}

In this section the model, derived by the method described in section 2.2 , is evaluated. First, it is shown that the modal decomposition gives a good understanding of the dynamic behaviour of a CMFM. A distinction is made between model results with and without a mass-flow. Second, the mass-flow measurement value is related to the mode shapes. Also, it is explained how the mass-flow is determined in practice by phase demodulation. In the third subsection, the effect of external vibrations on the flow measurement is shown, yielding a transfer function from external vibrations to the mass-flow measurement. The final subsection shows how the influence of broadband external vibrations on the RMS mass-flow measurement value can be calculated.

\subsubsection{Modal decomposition}

In section 2.2 a dynamic model of a CMFM is derived. From the model, mass and stiffness matrices are obtained. Solving the eigenvalue problem $\left(\boldsymbol{K}_{11}+\boldsymbol{N}_{11}-\omega_{i}^{2} \boldsymbol{M}_{11}\right) \boldsymbol{v}_{i}=0$, results in natural frequencies and the corresponding mode shapes. The vibrations of the CMFM can be obtained by superposition of these mode shapes. To gain more insight in the behaviour of the tube, the first eight mode shapes of the tube-window are depicted in Fig. 2.3. The first mode is a rotation of the tube-window around the $\theta_{\text {swing }}$-axis. Later it is shown that this mode is excited when there is a mass-flow, due to the Coriolis effect. Therefore, the first mode is termed a Coriolis mode. The excitation of this mode, due to a mass-flow, is not at 
the frequency of this mode, but at the actuation frequency. The second mode is termed an inplane mode, because it has no displacement in the direction of the sensors. The tube-window is actuated to oscillate in resonance around the $\theta_{\text {twist }}$-axis, so the third mode is termed the actuation mode. The fourth mode is also influenced by a Coriolis force and therefore termed the second Coriolis mode. The modes five and six are in-plane modes again. Mode seven is also a rotation around the $\theta_{\text {twist }}$-axis and therefore called the second actuation mode, although possible this mode is not used for actuation in our case. And finally, mode eight is again a Coriolis mode.

The reduced matrices of Eq. 2.4 with the first eight mode shapes $\left(\boldsymbol{V}=\left[\boldsymbol{v}_{1}, \boldsymbol{v}_{2}, \ldots, \boldsymbol{v}_{8}\right]\right)$ are derived. The reduced mass matrix is normalised to be the identity matrix:

$$
\boldsymbol{M}_{\mathrm{red}}=\boldsymbol{V}^{T} \boldsymbol{M}_{11} \boldsymbol{V}=\boldsymbol{I}
$$

The reduced stiffness matrix is a diagonal matrix, containing the natural frequencies:

$$
\boldsymbol{K}_{\mathrm{red}}=\boldsymbol{V}^{T}\left(\boldsymbol{K}_{11}+\boldsymbol{N}_{11}\right) \boldsymbol{V}=\operatorname{diag}\left(\omega_{1}^{2}, \omega_{2}^{2}, \ldots, \omega_{8}^{2}\right)
$$

The reduced velocity sensitive matrix, whereby the damping matrix $\boldsymbol{D}_{11}$ is omitted, is an skew-symmetric matrix:

$$
\begin{aligned}
\boldsymbol{C}_{\mathrm{red}} & =\boldsymbol{V}^{T} \boldsymbol{C}_{11} \boldsymbol{V} \\
& =\dot{\Phi}_{\mathrm{m}}\left[\begin{array}{rrrrrrrr}
-0.0000 & -0.0000 & -0.0279 & -0.0000 & -0.0000 & 0.0000 & 0.0047 & -0.0000 \\
0.0000 & -0.0000 & 0.0000 & -0.0000 & -0.0005 & 0.0000 & -0.0000 & 0.0000 \\
0.0279 & -0.0000 & 0.0000 & -0.0354 & -0.0000 & -0.0000 & 0.0000 & 0.0017 \\
0.0000 & 0.0000 & 0.0354 & -0.0000 & 0.0000 & -0.0000 & 0.0074 & 0.0000 \\
0.0000 & 0.0005 & 0.0000 & -0.0000 & 0.0000 & 0.0118 & 0.0000 & -0.0000 \\
-0.0000 & -0.0000 & 0.0000 & 0.0000 & -0.0118 & 0.0000 & -0.0000 & 0.0000 \\
-0.0047 & 0.0000 & -0.0000 & -0.0074 & -0.0000 & 0.0000 & 0.0000 & 0.0956 \\
0.0000 & -0.0000 & -0.0017 & -0.0000 & 0.0000 & -0.0000 & -0.0956 & 0.0000
\end{array}\right]
\end{aligned}
$$

The values of this matrix are proportional to the mass-flow $\dot{\Phi}_{\mathrm{m}}$ trough the fluid-conveying tube. When there is no flow, this matrix is zero and there is no coupling between the modes. But when there is a flow, this matrix describes the coupling between the modes. Because this coupling is proportional to the modal velocities and the fluid velocity, this is called the Coriolis effect. The tube-window is actuated to oscillate in resonance around the $\theta_{\text {twist }}$-axis, this results mainly in a modal velocity amplitude $\dot{z}_{3}$. The third column of $\boldsymbol{C}_{\text {red }}$, expressed in Eq. 2.11 is examined, we see that hereby also the modes 1,4 and 8 are influenced. Whereby the effect occurs at the actuation frequency $\omega_{3}$. Therefore, those modes are termed the Coriolis modes, as said before. Besides a mutual coupling between the symmetric and asymmetric out-of-plane modes, there is also a mutual coupling between the in-plane modes: mode 5 with mode 2 and 6. 


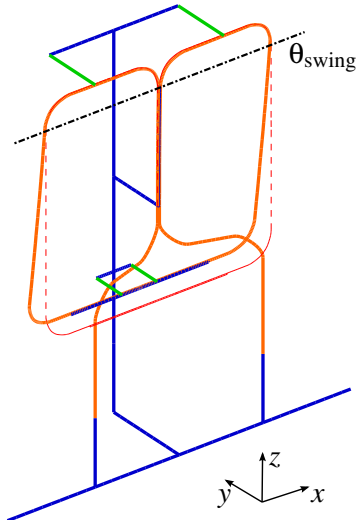

(a) Mode 1 - $39.7 \mathrm{~Hz}$ - Coriolis mode

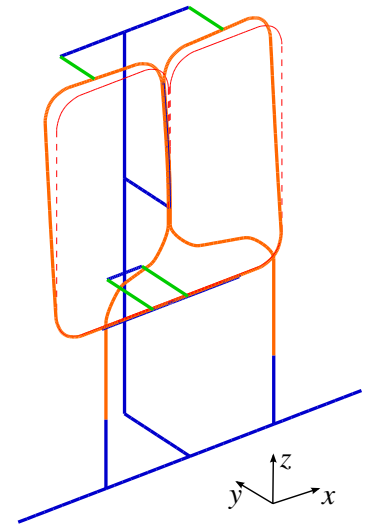

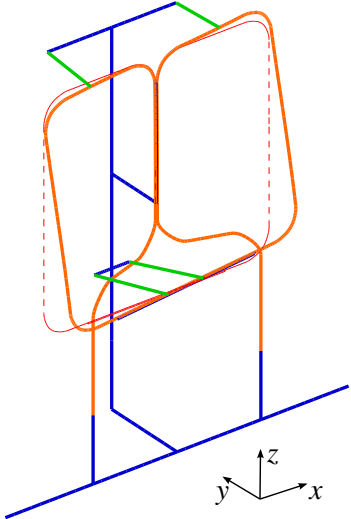

(b) Mode $2-60.3 \mathrm{~Hz}$ - Plane mode

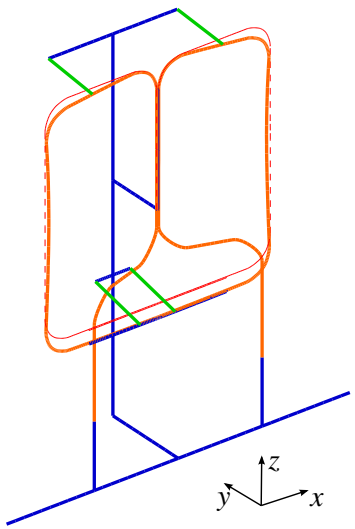

(d) Mode 4 - $188 \mathrm{~Hz}$ - Second (e) Mode 5 - $194 \mathrm{~Hz}$ - Plane Coriolis mode mode

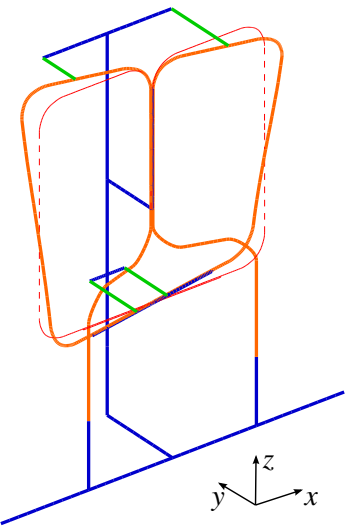

(g) Mode 7 - $353 \mathrm{~Hz}$ - Second actuation mode

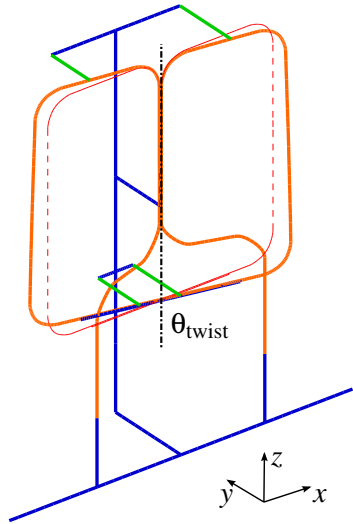

(c) Mode $3-87 \mathrm{~Hz}$ - Actuation mode

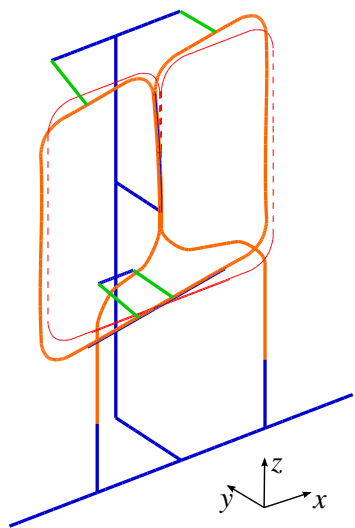

(f) Mode 6 - $274 \mathrm{~Hz}$ - Plane mode

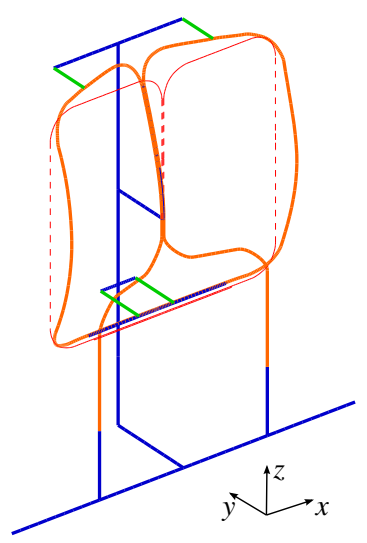

(h) Mode 8 - $525 \mathrm{~Hz}$ - Third Coriolis mode

Fig. 2.3 CMFM mode shapes with their corresponding natural frequencies, when the tube is filled with air

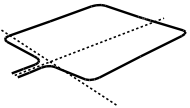




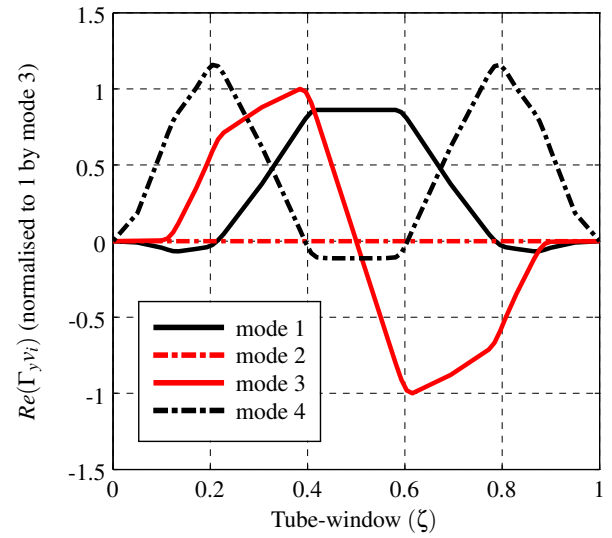

(a) Tube-window $y$-displacement as function of the tube-length $\zeta$, as shown in Fig. 2.2 for the first four mode shapes

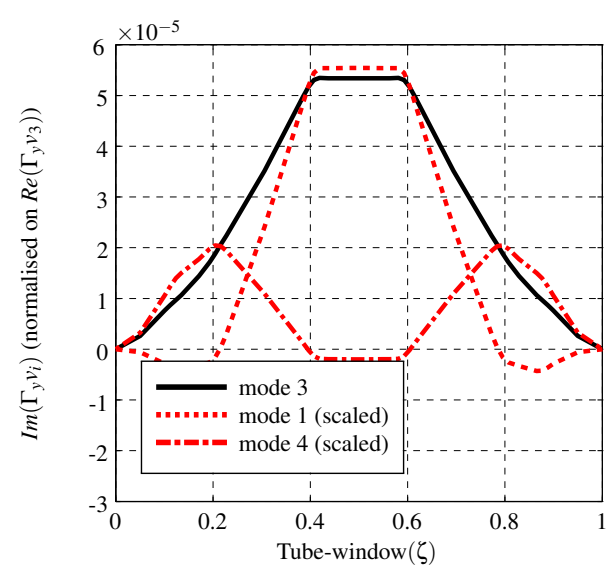

(b) Flow induced $y$-displacement as function of the tube-length $\zeta$. The amplitude is proportional to the mass-flow $\dot{\Phi}_{\mathrm{m}}$

Fig. 2.4 The mode shapes $\boldsymbol{\Gamma}_{y} \boldsymbol{v}_{i}$, where $\boldsymbol{\Gamma}_{y}$ is a vector with $y$-displacements of the tube-window-elements with respect to the model coordinates $\boldsymbol{q}$.

Predicting the Coriolis effect on tube displacements more accurate, is done by solving the quadratic eigenvalue problem:

$$
\left(\boldsymbol{K}_{11}+\boldsymbol{N}_{11}+j \omega_{i} \boldsymbol{C}_{11}-\omega_{i}^{2} \boldsymbol{M}_{11}\right) \boldsymbol{v}_{i}=0
$$

Several techniques to solve this problem are discussed by Cheesewright and Shaw [11]. They found that the eigenvalues $\omega_{i}$ are real and that the eigenvectors $\boldsymbol{v}_{i}$ are complex, because the mass, damping and stiffness matrices are positive definite and the velocity sensitive matrix $\boldsymbol{C}_{11}$ is skew-symmetric. The real part of the mode is the conventional modes for $\dot{\Phi}_{\mathrm{m}}=0$, while the imaginary part of the eigenvectors is the Coriolis distortion mode. The discussed techniques are unable to predict the Coriolis distortion modes accurately. We solved this issue by normalising the eigenvectors, such that $\boldsymbol{V}^{T} \boldsymbol{M}_{11} \boldsymbol{V}=\boldsymbol{I}$, resulting in a correct Coriolis distortion mode, independent of the technique used for solving the quadratic eigenvalue problem.

In Fig. 2.4(a) the real part of the tube-window $y$-displacement, determined from the eigenvectors, is depicted for the first four modes as function of the tube-window center-line $\zeta$ (see Fig. 2.2). Where the parameter $\zeta$ follows the trajectory of the curved tube-window, starting at the fixation point of the tube-window to the casing. The result is the same as shown in Fig. 2.3(a) (d) Furthermore, in Fig. 2.4(b) the flow induced part of mode 3 is depicted. This is the result of solving the quadratic eigenvalue problem of Eq.2.12

As suggested before, this flow induced mode can also be estimated by scaling the modes 1 and 4: $\alpha_{i} \cdot \Re\left(\Gamma_{y} v_{i}\right)$, whereby Eq. 2.4 is used to derive a scaling factor for those modes:

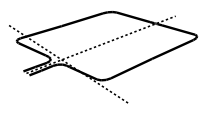




$$
\alpha_{i}=\frac{z_{i}}{z_{3}}=\frac{C_{\text {red }}(i, 3) \omega_{3}}{\omega_{3}^{2}-\omega_{i}^{2}} \mathrm{i}
$$

where $i$ is the mode to scale. Using Eq. 2.11 and the natural frequencies $\omega_{i}$, we see that only the modes 1 and 4 have a significant contribution to the flow induced mode. Both scaled modes are also shown in Fig. 2.4(b) The analysis above thus shows that a mass-flow only affects the out-of-phase component of the tube's motion, which can be reconstructed from scaling the other modes.

\subsubsection{Mass-flow measurement}

In the previous subsection the effect of a mass-flow on the mode shapes is shown. In this section it is discussed how a mass-flow can be measured using two displacement sensors $s_{1}$ and $s_{2}$. The tube-window is actuated to oscillate in its third eigen mode. In Fig. 2.4(a) we see the effect of actuation on the $y$-displacement of the tube-window. For $\zeta=0.5$, the displacement is zero, this is the rotation axis $\theta_{\text {twist }}$. In Fig. 2.2 we see that the sensors are placed on both sides of this rotation axis, resulting in a phase-difference between the sensor signals of $180^{\circ}$.

In Fig. 2.4(b) the flow induced vibration mode due to the Coriolis effect is depicted. The contribution to both sensor signals is equal in amplitude and phase, but this vibration mode occurs $90^{\circ}$ out of phase with the actuation mode, because it is the imaginary part of the mode. So, when a mass-flow is affecting the vibration mode of the tube-window, the phasedifference between the sensor signals $s_{1}$ and $s_{2}$ is not $180^{\circ}$ anymore, but is dependent on the mass-flow. The phase-difference between the two sensor signals is expressed as:

$$
\begin{aligned}
\Delta \phi=\arg \left(s_{1}\right)-\arg \left(s_{2}\right)+\pi & =\arctan \left(\frac{\mathfrak{I}\left(s_{1}\right)}{\mathfrak{R}\left(s_{1}\right)}\right)-\arctan \left(\frac{\mathfrak{I}\left(s_{2}\right)}{\mathfrak{R}\left(s_{2}\right)}\right) \\
& \approx \frac{\mathfrak{I}\left(s_{1}\right)}{\mathfrak{R}\left(s_{1}\right)}-\frac{\mathfrak{I}\left(s_{2}\right)}{\mathfrak{R}\left(s_{2}\right)} \approx 2 \frac{\mathfrak{I}\left(s_{1}+s_{2}\right)}{\mathfrak{R}\left(s_{1}-s_{2}\right)}
\end{aligned}
$$

where $s_{1}$ and $s_{2}$ represent the complex displacement amplitudes, calculated solving Eq. 2.12 Further the first approximation $(\arctan (x) \approx x)$ is valid for small radian angles only and for the second approximation is used that the two sensors are placed on equal distance of the rotation axis $\left(\mathfrak{R}\left(s_{1}\right) \approx \mathfrak{R}\left(-s_{2}\right)\right)$. The phase difference equation is made more distinct by defining two new displacements, based on the sensor signals:

$$
\begin{aligned}
& y_{\mathrm{act}}=\frac{1}{2}\left(s_{1}-s_{2}\right) \\
& y_{\mathrm{cor}}=\frac{1}{2}\left(s_{1}+s_{2}\right)
\end{aligned}
$$

where the differential-mode $s_{1}-s_{2}$ is named the actuation displacement $y_{\text {act }}$ and the commonmode $s_{1}+s_{2}$ the Coriolis displacement $y_{\text {cor }}$. This results in a new equation of the phase- 
difference (Eq. 2.14):

$$
\Delta \phi \approx 2 \frac{\mathfrak{I}\left(s_{1}+s_{2}\right)}{\mathfrak{R}\left(s_{1}-s_{2}\right)}=2 \frac{\mathfrak{I}\left(y_{\text {cor }}\right)}{\mathfrak{R}\left(y_{\text {act }}\right)}
$$

The approximation is valid for small flows, because then the Coriolis displacement is small compared to the actuation displacement. Another advantage of this new definition is the connection with the mode shapes, presented in the previous subsection. Using Eq. 2.7, the actuation displacement value is written as a combination of the modal displacements:

$$
\begin{aligned}
y_{\text {act }}=\frac{1}{2}\left(s_{1}-s_{2}\right) & =\frac{1}{2}\left(\boldsymbol{\Gamma}_{s_{1}}-\boldsymbol{\Gamma}_{s_{2}}\right) \boldsymbol{V} \boldsymbol{z} \\
& =\left[\begin{array}{llll}
-0.00 & 0.0028 .30-0.00-0.00-0.00-27.74-0.00
\end{array}\right] \boldsymbol{z}
\end{aligned}
$$

The actuation displacement is a combination of the modal amplitudes of the modes 3 and 7 . The actuation modes, as presented in Fig. 2.3. The same holds for the Coriolis displacement, which is a combination of the modes 1,4 and 8 :

$$
\begin{aligned}
y_{\text {cor }}=\frac{1}{2}\left(s_{1}+s_{2}\right) & =\frac{1}{2}\left(\boldsymbol{\Gamma}_{s_{1}}+\boldsymbol{\Gamma}_{s_{2}}\right) \boldsymbol{V} \boldsymbol{z} \\
& =\left[\begin{array}{lllll}
154.47 & 0.00-0.0021 .47 & 0.00-0.00 & 0.00-51.39
\end{array}\right] \boldsymbol{z}
\end{aligned}
$$

A controlled oscillation in the third mode results in excitation, proportional to the massflow $\dot{\Phi}_{\mathrm{m}}$, of the modes 1,4 and 8 with the third mode frequency, see Eq. 2.11. The Coriolis displacement is a combination those modal amplitudes and therefore this displacement is also proportional to the mass-flow. And, equally important, also proportional to the actuation displacement. This results in a phase difference, proportional to the mass-flow, but independent of the actuation displacement. A measurement sensitivity is defined as, the phase difference per unit mass-flow:

$$
S=\frac{\Delta \phi}{\dot{\Phi}_{\mathrm{m}}}(\operatorname{rad~s} / \mathrm{kg})
$$

The mass-flow is calculated from the measured phase difference and the measurement sensitivity. The measurement sensitivity $S$ is instrument, design, fluid density and temperature dependent. In case of large flows or in the transition between laminar and turbulent the relation is non-linear and thus the sensitivity becomes also flow dependent [1]. A numerical value of the measurement sensitivity is not given for the used instrument (Fig. 2.1), but the phase difference $\Delta \phi$ is also a valid measure for the mass-flow as these are related.

\subsubsection{Phase demodulation}

In practice the phase of the sensor signals is measured directly, without determining the amplitudes of the sensor signals. There are different digital signal processing methods that can be applied. A method is to apply dual quadrature demodulation, the method applied to a 
CMFM is described by Mehendale [44]. A phase-locked loop algorithm is implemented to compute the frequency $\omega_{\text {act }}=\omega_{3}$ of the oscillating tube. The filtered frequency is used to create two waveforms: a sine and a cosine. The measured sensor signal is multiplied with both waveforms and then filtered with a low-pass filter (LPF):

$$
\begin{aligned}
s_{1} \sin \left(\omega_{\text {act }} t\right) & =A_{1} \sin \left(\omega_{\text {act }} t+\phi_{1}\right) \sin \left(\omega_{\text {act }} t\right) \\
& =\frac{A_{1}}{2}\left(\cos \left(\phi_{1}\right)-\cos \left(2 \omega_{\text {act }} t+\phi_{1}\right)\right) \stackrel{\mathrm{LPF}}{\longrightarrow}=\frac{A_{1}}{2} \cos \left(\phi_{1}\right) \\
s_{1} \cos \left(\omega_{\text {act }} t\right) & =A_{1} \sin \left(\omega_{\text {act }} t+\phi_{1}\right) \cos \left(\omega_{\text {act }} t\right) \\
& =\frac{A_{1}}{2}\left(\sin \left(\phi_{1}\right)+\sin \left(2 \omega_{\text {act }} t+\phi_{1}\right)\right) \stackrel{\mathrm{LPF}}{\longrightarrow}=\frac{A_{1}}{2} \sin \left(\phi_{1}\right)
\end{aligned}
$$

This calculation thus results in two DC values, dependent on the phase difference $\phi_{1}$, between the sensor signal $s_{1}$ and the newly introduced waveform. Effectively the phase demodulation shifts the frequency of the sensor signals by the actuation frequency. The phase, independent of the amplitude $A_{1}$ of the sensor signals, is calculated as follows:

$$
\frac{\frac{A_{1}}{2} \sin \left(\phi_{1}\right)}{\frac{A_{1}}{2} \cos \left(\phi_{1}\right)}=\tan \phi_{1} \rightarrow \phi_{1}
$$

The same is done for the second sensor, resulting in $\phi_{2}$. This results in a phase difference between the two sensor signals:

$$
\Delta \phi=\phi_{1}-\phi_{2}
$$

The phase difference divided by the measurement sensitivity (Eq. 2.20) results in an estimation of the mass-flow.

The low pass filter is the key in the trade-off between speed of the flow measurement and the measurement noise. A lower cut-off frequency reduces the amount of measurement noise, but also reduces the response time.

\subsubsection{Transmissibility external vibrations}

In the previous subsection we showed that the Coriolis displacement is a measure to calculate the mass-flow. In this section we show that besides a mass-flow, external vibrations result in a Coriolis displacement. The influence of external vibrations on the Coriolis displacement, expressed in the Laplace $s$-domain, is equal to:

$$
y_{\mathrm{cor}}(s)=\boldsymbol{T}_{y_{\mathrm{cor}, a_{0}}}(s) \boldsymbol{a}_{0}(s)
$$

where $\boldsymbol{T}_{y_{\mathrm{cor}, a_{0}}}(s)$ is determined using the State Space model (Eq. 2.8) and the Coriolis displacement definition (Eq. 2.16). The model has in total 6 inputs, 3 translations and 3 rotations, 


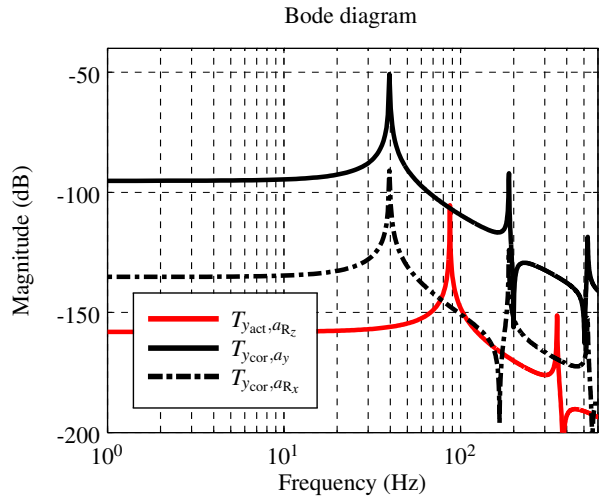

Fig. 2.5 Transmissibility of external vibrations to the Coriolis and actuation displacement (The rest of the transfer functions have a gain lower then $-300 \mathrm{~dB}$ )

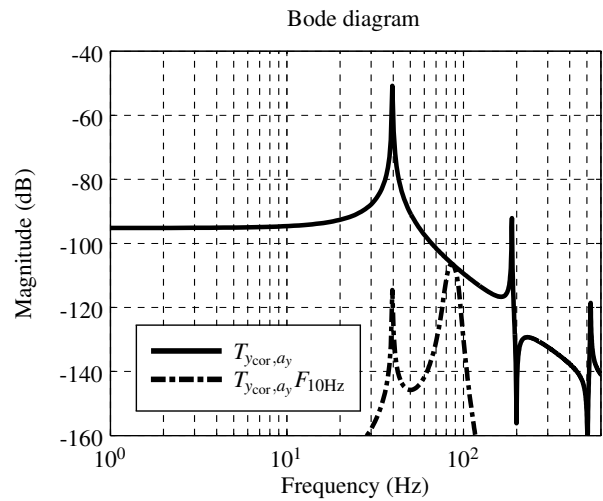

Fig. 2.6 Transmissibility of external vibrations in the dominant direction $a_{y}$ to a Coriolis displacement, with and without the phase demodulation including a $10 \mathrm{~Hz}$ band-pass filter.

combined in one vector $\boldsymbol{a}_{0}=\left\{a_{x}, a_{y}, a_{z}, \alpha_{\mathrm{R}_{x}}, \alpha_{\mathrm{R}_{y}}, \alpha_{\mathrm{R}_{z}}\right\}^{T}$. Besides the Coriolis displacement as an output, we also define the actuation displacement (Eq.2.15) as output. The MIMO system has 2 outputs and 6 inputs. This is a model with in total 12 transfer functions. The transmissibility functions of external accelerations $\boldsymbol{a}_{0}$ to the actuation and Coriolis displacements are depicted in Fig. 2.5

The figure only shows three transfer functions, instead of the 12 we calculated. This is because the magnitude of the other nine is below $-300 \mathrm{~dB}$, which is approximatively zero, compared to the three remaining transfer functions.

The Coriolis displacement is influenced by a translation in $y$-direction and a rotation around the $x$-axis. Resonance frequencies are visible at the Coriolis modes (39.7, 188 and $525 \mathrm{~Hz}$ ). And the actuation displacement is influenced by a rotation around the $z$-axis. In the transfer function, resonance frequencies are visible at the actuation modes. (87 and $353 \mathrm{~Hz}$ ). The different modes are depicted in Fig. 2.3 Those three dominant directions can be explained using the couplings matrix between the modes of mechanism and the input vector:

$$
\begin{aligned}
\boldsymbol{V}^{T} \boldsymbol{M}_{12} \boldsymbol{a}_{0} & =\boldsymbol{F}_{\mathrm{dis}} \boldsymbol{a}_{0} \\
& =\left[\begin{array}{rrrrrr}
0.00000 & -0.00703 & 0.00000 & 0.00007 & 0.00000 & -0.00000 \\
-0.00684 & -0.00000 & -0.00001 & -0.00000 & -0.00010 & 0.00018 \\
0.00000 & 0.00000 & 0.00000 & -0.00000 & 0.00000 & -0.00013 \\
0.00000 & 0.00598 & -0.00000 & 0.00015 & 0.00000 & -0.00000 \\
-0.00003 & 0.00000 & 0.00837 & 0.00022 & 0.00000 & 0.00000 \\
0.00425 & 0.00000 & 0.00003 & 0.00000 & -0.00015 & -0.00011 \\
-0.00000 & 0.00000 & 0.00000 & -0.00000 & -0.00000 & -0.00002 \\
0.00000 & 0.00151 & 0.00000 & 0.00003 & -0.00000 & -0.00000
\end{array}\right] \boldsymbol{a}_{0}
\end{aligned}
$$


The Coriolis displacement, see Eq.2.19 measures only the displacements of the first, fourth and eighth mode. When we look at the rows 1, 4 and 8, we see that there are only non-zero values in the columns two and four. This indicates that the Coriolis displacement is only influenced by a translation in $y$-direction and a rotation around the $x$-axis.

The phase difference is a function of the Coriolis and actuation displacement (Eq. 2.17). Those displacements is not only introduced by the actuation and due to a mass-flow, but also by external vibrations, resulting in an erroneous phase difference:

$$
\Delta \phi(s)=2 \frac{y_{\text {cor }}(s)}{y_{\text {act }}(s)} \approx \frac{2}{\left|y_{\text {act }}\right|} \boldsymbol{T}_{y_{\mathrm{cor}}, a_{0}}(s) \boldsymbol{a}_{0}(s)
$$

where $\left|y_{\text {act }}\right|$ is the amplitude of the actuation mode. This amplitude is kept constant, using feedback control.

\subsubsection{Measurement error}

In section 2.3.2.1, is explained how a phase difference between the sensor signals is calculated, using phase demodulation. In the frequency domain, this is similar to a bandpass filter around frequency $\omega_{3}$ and a frequency shift. First, we add the bandpass filter to Eq. 2.27 and obtain a transmissibility of external vibrations to the phase difference:

$$
\boldsymbol{T}_{\Delta \phi, \boldsymbol{a}_{0}}(s)=\frac{2}{\left|y_{\mathrm{act}}\right|} \boldsymbol{T}_{y_{\mathrm{cor}}, \boldsymbol{a}_{0}}(s) F(s)
$$

where $F(s)$ is a band-pass filter with a bandwidth two times the cut-off frequency of the lowpass filter, used in the phase demodulation algorithm. In Fig.2.6 the dominant transmissibility including a $10 \mathrm{~Hz}$ band-pass filter around the frequency $\omega_{3}$ is depicted.

The external vibrations can be a broadband disturbance and the output is a low-frequent measurement value, due to the frequency shift. The cumulative influence is investigated by looking to the cumulative mean square response over the whole frequency range $v$, which is given by:

$$
\sigma_{\Delta \phi}^{2}=\int_{-\infty}^{\infty}\left|\boldsymbol{T}_{\Delta \phi, a_{0}}(v)\right|^{2} \boldsymbol{\Phi}_{a_{0}}(v) \mathrm{d} v
$$

where $\boldsymbol{\Phi}_{\boldsymbol{a}_{0}}$ is the Power Spectral Density (PSD) function of the disturbance and $\boldsymbol{T}_{\Delta \phi, \boldsymbol{a}_{0}}$ the modelled transmissibility of external vibrations to a phase difference, as partly depicted in Fig. 2.6

Due to the low-pass filter in the phase demodulation, only disturbances around the actuation frequency and the Coriolis frequency have an influence on the phase difference, see Fig. 2.6. The result is that a disturbance with a frequency close to the actuation frequency has a direct impact on a mass-flow reading. In the following section the modelled influence of external vibrations on a mass-flow measurement value is validated experimentally. 


\subsection{Model validation}

In this section the modelled influence of external vibrations on a mass-flow measurement is validated. The first subsection explains the experimental setup. Second, the transmissibility functions of external vibrations to the actuation and Coriolis displacement are validated. Third, the influence of broadband external vibrations on the mass-flow measurement value is compared with the estimated value from the model.

\subsubsection{Experimental setup}

To estimate the transmissibility functions, the reference instrument is mounted on a 6-DOF vibration isolation setup (Fig. 2.77. The platform is suspended at a low frequency in all directions (about $22 \mathrm{~Hz}$ ), using a Stewart type platform, mounted on a rigid floor plate. The platform is actuated by six voice coil actuators. For each direction, the vibrations of the platform are measured using accelerometers. Using a rigid body model, the relation between the sensor coordinates $\boldsymbol{q}$ and Cartesian coordinates $\boldsymbol{x}=\left\{x, y, z, \theta_{x}, \theta_{y}, \theta_{z}\right\}^{T}$, is derived as:

$$
\boldsymbol{x}=\boldsymbol{R} \boldsymbol{q}
$$

The accelerometers on the platform are colocated with the voice coil actuators. Therefore, the matrix $\boldsymbol{R}^{-1}$ is used also to apply forces in the Cartesian coordinate system. The inverse is possible because the use of 6 sensors and 6 Cartesian coordinates. More details of the experimental setup are given by Tjepkema [64].

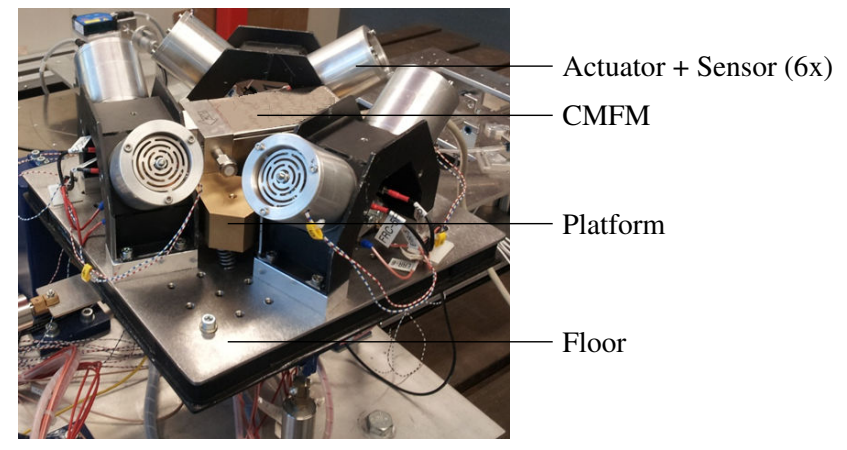

Fig. 2.7 Shaker setup - The CMFM (Fig. 2.1) is mounted on a Stewart platform. Voice coil actuators are used to apply forces on the low frequent $(22 \mathrm{~Hz})$ suspended platform and accelerometers are used to measure the platform vibrations $\boldsymbol{a}_{0}$. 
The measurements are performed using a National Instruments NI4472 card using a 24 $\mathrm{kHz}$ sample-rate with 24-bit resolution. To determine the transmissibilities, the platform is excited using the voice coil motors as shakers. These shakers provide a multi-sine signal, containing frequencies between 1 and $550 \mathrm{~Hz}$. Accelerometers (Endevco 7703A-1000) on the platform body are used to measure the input disturbance $\boldsymbol{a}_{0}$, while optic sensors inside the CMFM are used to measure the tube-window displacement.

Power Spectral Densities (PSD) of the different discrete-time signals are estimated via Welch's method. To apply the method, we use the Matlab function pwelch. In total a dataset of $60 \mathrm{~s}$ of measurement is used. To reduce the noise level, the method uses a $24 \mathrm{k}$-point symmetric Hanning window. The performance is evaluated by the transmissibility function. This transfer function is estimated with the Matlab function tfestimate. Again, using a dataset of $60 \mathrm{~s}$ and a 24k-point symmetric Hanning window.

\subsubsection{Transmissibility}

In this subsection the transmissibilities of external vibrations to the actuation and Coriolis displacement are validated. The modelled functions are given in section 2.3.3. First, the three dominant directions are estimated and second, we validate that the three functions are sufficient enough to describe the sensitivity of external vibrations.

\subsubsection{Dominant directions}

In section 2.3.3 we explained that are three dominant directions of the 6 external vibrations to the actuation and Coriolis displacement. In this section, we validate those three directions, using the setup described in section 2.4.1. The actuators excite the platform with a random signal in one direction only and the sensors measure the displacement of the tube-window and the accelerations of the platform. Based on both datasets a transfer function is estimated. This is done for all three dominant directions.

The results of the three experiments are given in Fig. 2.8. The modelled results agree well with the experimental results. Clearly visible are the undamped resonance frequencies of 42 and $88 \mathrm{~Hz}$ and the gain is as expected. The estimated transmissibility $T_{y_{\mathrm{cor}}, \alpha_{\mathrm{R} x}}$ shows an extra peak at $22 \mathrm{~Hz}$. This is a resonance frequency of the platform. In this measurement the platform is not only rotating, but also translating in $y$-direction with frequencies mainly around the suspension frequencies. Because the transmissibility $T_{y_{\mathrm{cor}}, a_{y}}$ is larger than $T_{y_{\mathrm{cor}}, \alpha_{\mathrm{R}_{x}}}$, we cannot assume an uncoupled system and we see the effect in the estimation of $T_{y_{\text {cor }}, \alpha_{\mathrm{R}_{x}}}$.

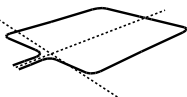



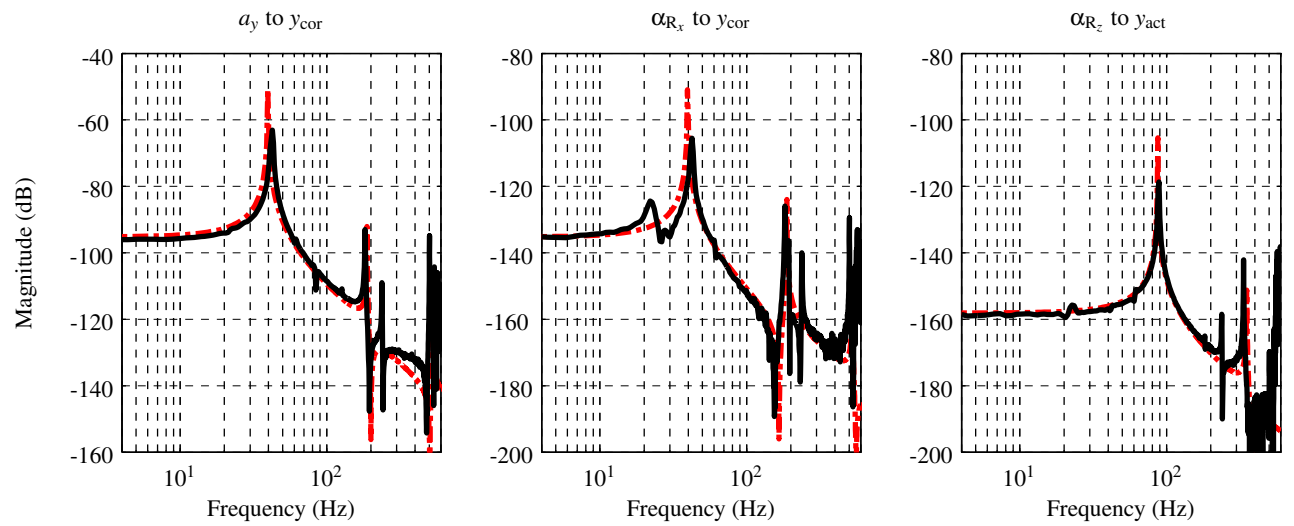

Fig. 2.8 Transmissibility of external vibrations to the actuation and Coriolis displacement. Experimental results (- ${ }^{-}$are compared to the modelled results (-----), as given in Fig. 2.5

\subsubsection{Broadband 3D disturbance}

In the previous subsection we showed three dominant directions. Now, we need to know if those three functions are indeed the important directions. Therefore, we apply a frequency dependent force in all six directions and measure the actuation and Coriolis displacement, and the six disturbances. Now, the measured and the estimated displacement can be compared. The frequency content of a signal can be described by its Power Spectral Density (PSD), so we will compare the PSDs of both signals. The estimated PSDs are calculated as:

$$
\begin{aligned}
& \hat{\boldsymbol{\Phi}}_{y_{\mathrm{act}}}=\left|\boldsymbol{T}_{y_{\mathrm{act}}, \boldsymbol{a}_{0}}\right|^{2} \boldsymbol{\Phi}_{\boldsymbol{a}_{0}} \approx\left|T_{y_{\mathrm{act}}, \alpha_{\mathrm{R}_{z}}}\right|^{2} \boldsymbol{\Phi}_{\alpha_{\mathrm{R}_{z}}} \\
& \hat{\boldsymbol{\Phi}}_{y_{\mathrm{cor}}}=\left|\boldsymbol{T}_{y_{\mathrm{cor}}, \boldsymbol{a}_{0}}\right|^{2} \boldsymbol{\Phi}_{a_{0}} \approx\left|T_{y_{\mathrm{cor}}, a_{y}}\right|^{2} \boldsymbol{\Phi}_{a_{y}}+\left|T_{y_{\mathrm{cor}}, \alpha_{\mathrm{R}_{x}}}\right|^{2} \boldsymbol{\Phi}_{\alpha_{\mathrm{R}_{x}}}
\end{aligned}
$$

The actuation displacement is, according to the model, only dependent on an $R_{z}$-disturbance, while the Coriolis displacement is dependent on a $y$ - and $\mathrm{R}_{x}$-disturbance. The PSDs $\boldsymbol{\Phi}_{a_{0}}$ of the applied external vibrations are shown in Fig. 2.10 (Experiment 4). This is an approximately broadband flat disturbance in all translation and rotational directions. Clearly visible are the resonance frequencies of the platform. The disturbance is acquired with a multi-sine disturbance between 1 and $550 \mathrm{~Hz}$. For comparison, the Vibration Criterion (VC) curves [23] are added. The VC-curves are meant as upper bounds for the peaks in the external vibration spectrum. The applied broadband disturbance is not a realistic external vibration, but is used to compare different CMFMs.

In Fig. 2.9 the measured PSDs of the actuation and Coriolis displacements are compared to the estimated ones (Eq.2.31 and 2.32), which are similar. For $\hat{\boldsymbol{\Phi}}_{y_{\text {act }}}$, the largest difference is that there are resonance frequencies visible at 42, 61, 88 and $183 \mathrm{~Hz}$. This clearly are modes of the system and do have an influence on the actuation displacement. In the model they do 

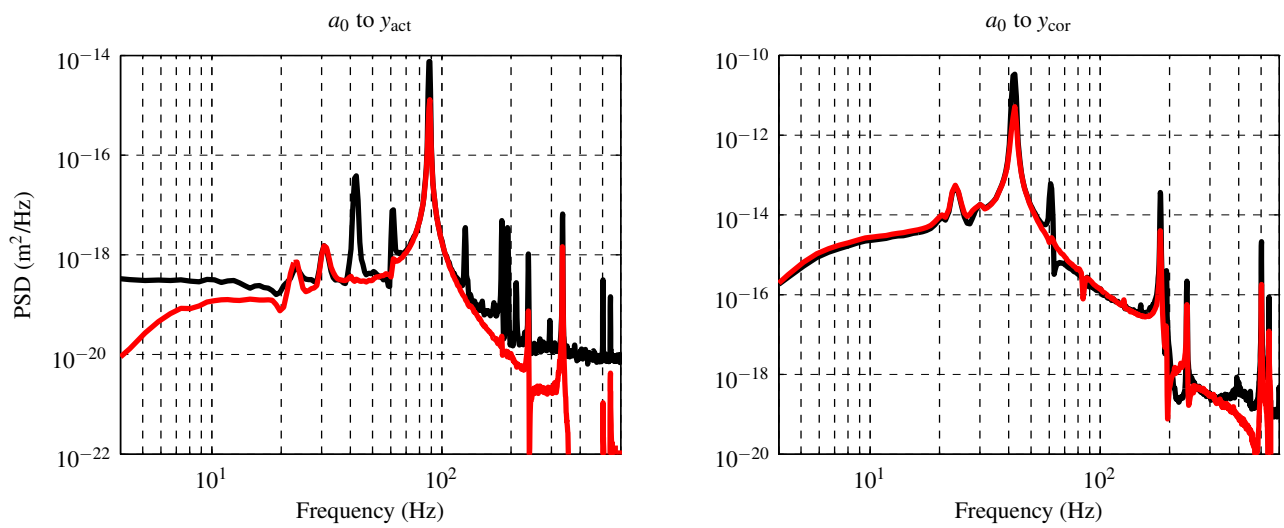

Fig. 2.9 Measurement (—) compared to the estimation (— $\longrightarrow$ ), based on Eq. 2.31 and 2.32

Table 2.1 Comparison of natural frequencies between model and experiment

\begin{tabular}{lccl}
\hline Mode & Model & $\begin{array}{c}\text { Experiment } \\
f(\mathrm{~Hz})\end{array}$ & Description \\
\hline 1 & $f(\mathrm{~Hz})$ & 42.2 & Coriolis mode \\
2 & 39.7 & 61.1 & In plane mode \\
3 & 60.3 & 88.3 & Actuation mode \\
4 & 87.0 & 183 & Second Coriolis mode \\
5 & 188 & 193 & In plane mode \\
6 & 194 & 238 & In plane mode \\
7 & 274 & 335 & Second actuation mode \\
8 & 353 & 500 & Third Coriolis mode \\
\hline
\end{tabular}

not turn up, because we assumed perfect sensors: a pure $y$-displacement and an equal sensor gain. At low and high frequencies the noise-level of the sensor is visible.

In practice the actuation displacement is of less importance, because the actuated displacement is much larger than the effect of external disturbances. For the mass-flow measurement, the Coriolis displacement is more important.

The other approximation $\hat{\boldsymbol{\Phi}}_{y_{\text {cor }}}$ is better. The power density is equal, except a pole-zero cancellation is visible at $61 \mathrm{~Hz}$. This is an in-plane mode of the tube-window (Fig. 2.3(b)). This comparison confirms that there are indeed only two dominant directions, regarding the Coriolis displacement.

Another result of this experiment is that the resonance frequencies of the tube-window are estimated. The comparison, for the first eight resonance frequencies, is given in Table 2.1 This comparison confirms that there is a good dynamical model available. 


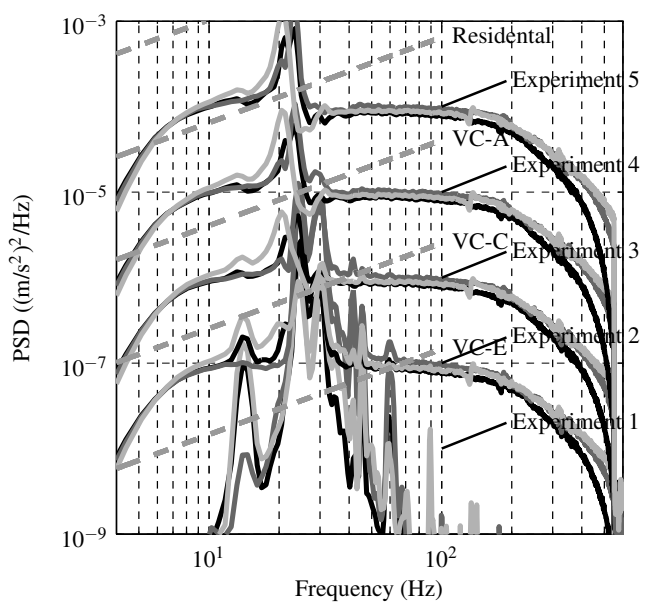

(a) Translational disturbances - $a_{x}, a_{y}, a_{z}$

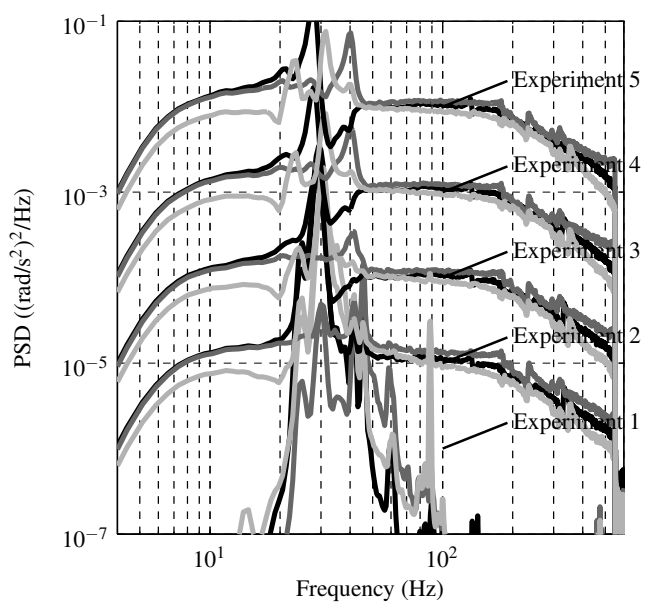

(b) Rotational disturbances $-\alpha_{\mathrm{R}_{x}}, \alpha_{\mathrm{R}_{y}}, \alpha_{\mathrm{R}_{z}}$

Fig. 2.10 Measured external vibration PSDs $\boldsymbol{\Phi}_{a_{0}}$ of 5 experiments compared to VC-curves to show the magnitude of the disturbance. The applied disturbances are relatively large in comparison to real floor spectra. $x(\longrightarrow), y(\longrightarrow), z(-)$

Table 2.2 RMS measurement error due to applied disturbance (Fig.2.10), values are normalised by the first value.

\begin{tabular}{lccc}
\hline Experiment & $\begin{array}{c}\text { Disturbance } a_{z} \\
\text { RMS }\left(\mathrm{m} / \mathrm{s}^{2}\right)\end{array}$ & $\begin{array}{c}\text { Measurement value instrument } \\
\text { RMS }(\mathrm{rad})\end{array}$ & $\begin{array}{c}\text { Model estimation } \sigma_{\Delta \phi} \\
(\text { Eq. [2.29) }(\mathrm{rad})\end{array}$ \\
\hline 1 & 0.0078 & 1.000 & 0.2045 \\
2 & 0.0091 & 2.037 & 1.831 \\
3 & 0.0174 & 5.680 & 5.745 \\
4 & 0.0527 & 17.63 & 18.25 \\
5 & 0.1684 & 56.20 & 56.81 \\
\hline
\end{tabular}

\subsubsection{Flow error}

In section 2.3.4, we explained how the influence of external vibrations cumulatively add up to the RMS measurement error. In this subsection we apply disturbances in all directions with a different magnitude. Then we compare the RMS measurement value with the estimated one (Eq. 2.29), to validate the influence of external vibrations. The PSDs of several experiments, with a multi-sine disturbance between 1 and $550 \mathrm{~Hz}$, are given in Fig. 2.10. Using this data, $\sigma_{\Delta \phi}$ is calculated. The results are given in Table 2.2. For experiment 2-5 the estimation is consistent with the measured value. In the first experiment the measured value is comparable to the noise floor of the measurement value and the estimation shows that the noise floor is larger than the effect of external vibrations.

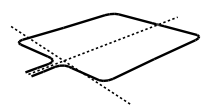


Referring back to Fig. 2.6, we see that the influence is mainly due to external disturbances with frequencies around the Coriolis $(42 \mathrm{~Hz})$ and the actuation frequency of $88 \mathrm{~Hz}$. The effect around the actuation frequency can only be reduced, without affecting the mass-flow measurement, by lowering the transmissibility function $\boldsymbol{T}_{y_{\mathrm{cor}}, \boldsymbol{a}_{0}}$.

\subsection{Discussion}

In section 2.4 we validated the model and saw that we can quantitatively estimate the influence of external vibrations on the mass-flow measurement. In this section we show first that the number of relevant degrees of freedom can be reduced further and secondly how the measurement error can be reduced.

\subsubsection{Direction dependency}

Section 2.4.2 showed that the Coriolis displacement is influenced by one translational and one rotational external vibration. Especially for the rotational input, the point where the external accelerations are imposed is important. In Fig. 2.11(a) the front view of the CMFM FEM model (Fig. 2.2) is shown. At the bottom, the coordinate system $x y z$ is given. A $y$ translation and $\mathrm{R}_{x}$-rotation result both in a Coriolis displacement, which is also an out-ofplane $y$-translation.

The flow induced displacement is mainly due to the first Coriolis mode shape, see Fig. 2.4(b) In Eq. 2.26, we saw that this mode is actuated by a $y$-translation and $\mathrm{R}_{x}$-rotation, due to the non-zero values in the columns two and four, on the first row. Now, we can translate the coordinate system $x y z$ with a $z$-displacement, resulting in the new coordinate system $x^{\prime} y^{\prime} z^{\prime}$ (see Fig. 2.11(a). Then again the matrix $\boldsymbol{F}_{\text {dis }}$ is calculated to see the effect of external vibrations on the first mode. The effect of a $\mathrm{R}_{x}$-rotation on the first mode is shown in Fig. 2.11(b), by means of $\boldsymbol{F}_{\text {dis }}(1,4)$. The result shows that when the coordinate system $x y z$ is translated by $\frac{z}{h} \approx 0.4$, the influence of a $\mathrm{R}_{x}$-rotation on the first mode is approximating zero. Whereby the location is dependent on the dimensional properties of the tube-window. Note that thus the measurement of external vibration in the $y$-direction on this new point is sufficient to quantitatively estimate the RMS mass-flow error.

Therefore, the influence of external vibrations can be approximated quite well by a 1D model of one translational external vibration to the Coriolis displacement. This can be done with a mass-spring model, containing the modal mass and stiffness of the first Coriolis mode. 


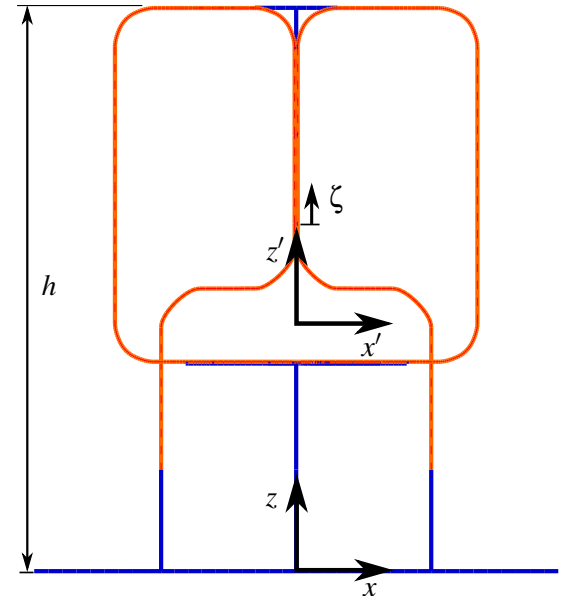

(a) Front view of the FEM model of the CMFM. The instrument has a height $h$ and external vibrations are imposed at the origin of the coordinate system $x y z$.

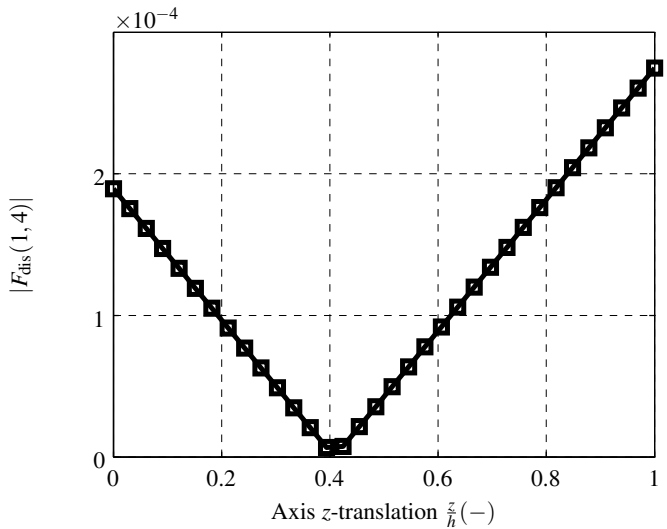

(b) Influence of a $\mathrm{R}_{x}$-rotational disturbance on the first mode shape, by depicting $\boldsymbol{F}_{\text {dis }}(1,4)($ Eq. 2.26 as function of the displacement

Fig. 2.11 Influence of shifting the coordinate system $x y z$

\subsubsection{Reducing influence of external vibrations}

The influence of external vibrations can be estimated, using the disturbance $\boldsymbol{\Phi}_{a_{0}}$ and the transmissibility $\boldsymbol{T}_{\Delta \phi, a_{0}}$ (Eq. 2.29):

$$
\sigma_{\Delta \phi}^{2}=\int_{-\infty}^{\infty}\left|\boldsymbol{T}_{\Delta \phi, \boldsymbol{a}_{0}}(\mathrm{v})\right|^{2} \boldsymbol{\Phi}_{a_{0}}(\mathrm{v}) \mathrm{d} \boldsymbol{v}=\int_{-\infty}^{\infty}\left|\frac{2}{\left|y_{\mathrm{act}}\right|} \boldsymbol{T}_{y_{\mathrm{cor}}, \boldsymbol{a}_{0}}(\mathrm{v}) F(\mathrm{v})\right|^{2} \boldsymbol{\Phi}_{\boldsymbol{a}_{0}}(\mathrm{v}) \mathrm{d} \boldsymbol{v}
$$

Reducing the disturbance $\boldsymbol{\Phi}_{a_{0}}$ by implying stringent requirements on the surroundings is not possible in many applications. Therefore, the transmissibility $\boldsymbol{T}_{\Delta \phi, \boldsymbol{a}_{0}}$ should be minimal, implying a good filter algorithm and/or mechanical design of the instrument.

The transmissibility can be reduced by applying passive vibration isolation. Passive isolation consist of several stages of mass-spring-damper systems between the floor and the casing of a machine [53]. The parameters should be adjusted to achieve high-frequency attenuation. The reduction of the transmissibility will be subject of future research. 


\subsection{Conclusions}

In this study a model of a CMFM is derived to understand and quantify the influence of external vibrations on the mass-flow measurement. In an experiment predefined vibrations are applied on the casing of the CMFM and the RMS measurement error is determined. The experimental results correspond well on a qualitative and quantitative level with the modelled results.

The result is an significant extension of the work of Cheesewright [13], not only the frequencies are shown where the CMFM is sensitive for external vibrations, but also a quantitative estimation of the expected mass-flow error is given, based on the modelled transmissibility function.

The agreement between model and measurements implies firstly that the influence of any external vibration spectrum on the flow error, with some limitations due to linearity of the model, can be estimated. Thereby, the suitability of a certain location for the placement of a CMFM can be determined. Secondly, the insight in the relation between vibration spectra and the flow error, the transmissibility, can be used to compare the performance of different CMFM designs and to optimise the performance by shaping their respective transfer functions.

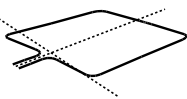




\title{
Chapter 3
}

\section{Active vibration isolation feedback control}

\begin{abstract}
Active Vibration Isolation Control (AVIC) can be used to reduce the transmissibility of external vibrations to internal vibrations. In this paper a proposal is made for integrating AVIC in a Coriolis Mass-Flow Meter (CMFM). Acceleration feedback, virtual mass and virtual skyhook damping are added to a passively suspended CMFM, resulting in a lowered suspension frequency. In addition, position feedback of the internal deformation is used to damp the internal mode. The controller design is based on a simplified model and is validated on an experimental setup. Results show that the sensitivity to external vibrations is reduced by $40 \mathrm{~dB}$ for the RMS measurement error.
\end{abstract}

This chapter is reprinted from: L. van de Ridder, M. A. Beijen, W. B. J. Hakvoort, J. van Dijk, J. C. Lötters, and A. de Boer. Active vibration isolation feedback control for coriolis mass-flow meters. Control Engineering Practice, 33C:76-83, 2014. URL http://dx. doi. org/10.1016/j.conengprac.2014.09.007, with permission from Elsevier. 


\subsection{Introduction}

A Coriolis Mass-Flow Meter (CMFM) is an active device based on the Coriolis force principle for direct mass-flow measurements, with high accuracy, range-ability and repeatability [1]. The working principle of a CMFM is as follows: a fluid conveying tube is actuated to oscillate at a low amplitude, whereby a resonance frequency is used to minimise the amount of required energy. A fluid-flow in the vibrating tube induces Coriolis forces, which are proportional to the mass-flow, and affect the tube motion resulting in a change of the mode shape. Measuring the tube displacement in such a way that the change of its mode shape is determined, allows calculating the mass-flow.

External vibrations create additional components in the CMFM sensor signals and such additional components can introduce a measurement error [14]. For low flows, the Coriolis force induced motion is relatively small compared to motions induced by external vibrations, thus CMFMs designed to be sensitive to low flows are rather sensitive to external vibrations. The effect of external vibrations on the sensor response of a CMFM has been studied previously in Chapter 2 This study showed that external vibrations around the meter's drive and Coriolis frequencies produce a measurement error, regardless of the phase detection algorithm used. A quantitative estimation of the expected mass-flow error in response to external vibrations can be obtained from the transmissibility function (Chapter 2).

To reduce the sensitivity of the flow measurement with respect to external vibrations, passive or active vibration isolation can be used. Passive isolation consists of several stages of mass-spring-damper systems between the floor and the casing of a machine [53]. The parameters are adjusted to achieve high-frequency attenuation, which is suitable for many applications. However, the performance of passive isolation applied to a CMFM is limited due to a minimal suspension frequency, which is caused by the parasitic stiffness of the fluidconnecting-tubes and the maximum allowable gravitational sagging of the stage. Those large deformations in the fluid-connecting-tubes result in high internal stresses. An alternative approach is to apply active vibration isolation control to a flexible-suspended single-tube configuration. This is an application of active hard mount isolation for precision equipment [64].

In this paper, active vibration isolation is applied to a CMFM is investigated, to optimise the shape of the transmissibility function of a CMFM from external vibrations to the measurement value. The objectives of the present work are summarised as follows:

1. Shaping the transmissibility from external vibrations to the internal displacement by actively reducing the suspension mode to lower the transmissibility at the actuation frequency of the CMFM.

2. Adding damping to the internal deformation mode, the Coriolis mode, to limit the effect of shock disturbances.

3. Avoiding that the flow measurement sensitivity changes.

A control algorithm that satisfies the stated objectives is presented and tested. The algorithm is a combination of known feedback strategies for vibration isolation [34, 77, 52]. The novelty is the smart combination of those algorithms: acceleration feedback, velocity feedback, internal mode damping, cascade control and loop shaping; tuned, modified and applied to the specific application of Coriolis Mass-Flow Meters. Notably, the requirement that the 
flow measurement should not be affected, requires some dedicated modifications to the algorithms. The algorithm is not yet integrated within a commercial CMFM, but implemented at a test setup, consisting of an external isolator platform and a CMFM. Therefore, we present a proof-of-principle of the feasibility of integrating active vibration control into the design of CMFMs.

In section 3.2, the reference instrument and the experimental test-setup are discussed briefly, including the model describing the influence of external vibrations. In section 3.3. a simplified dynamic model of the essential dynamics is presented and a control algorithm is designed to meet the objectives stated above, the main contribution of this paper. Experimental results of the proposed control design that validate the model are presented in section 3.4 The results are discussed in section 3.5 and section 3.6 finalises this paper by drawing conclusions.

\subsection{Material}

In this section, the materials used and the analysis methods are described. In the first subsection, a model of a CMFM is presented and is further explained; how the external vibrations affect the measurement value. A vibration isolator setup is presented in section 3.2.2, which will be used to provide external vibrations and active vibration isolation to the casing of a CMFM. In the final subsection a simplified model is given, consisting of the CMFM and the vibration isolator setup, to meet the performance objectives.

\subsubsection{Reference instrument}

For this research a functional model of the patented design [45, 44] (see Fig. 3.1) is used. First, a Finite Element Method (FEM) model is derived, using the multi-body package SPACAR [31]. The graphical representation of the model is shown in Fig. 3.2, The model consists of a tube, conveying the fluid flow, which is actuated by two actuators act $_{1}$ and act $_{2}$. The moving part of the tube has a rectangular shape and is referred to as the tube-window. The displacements of the flexible tube-window are measured by two displacements sensors $s_{1}$ and $s_{2}$. On the casing a vector $\boldsymbol{a}_{0} \in \mathbb{R}^{6 x 1}$, representing the external vibrations and consisting of three translations and three rotational movements, is imposed. The model is made out of multi-body beam, truss and tube elements [47]. The beam elements are used to model the rigid casing and the truss elements to measure relative displacements and to apply a force on the tube-window. Further, a tube-element is used to model the inertial interaction between the flow and the tube dynamics.

The mass, damping and stiffness matrices are the result of the multi-body model, which are used to compute the mode-shapes and resonance frequencies of the tube-window. The first mode is a rotation of the tube-window around the $\theta_{\text {swing-axis, with a natural frequency of }}$ $39 \mathrm{~Hz}$. This first mode is termed a Coriolis mode, because this mode is mainly excited when 


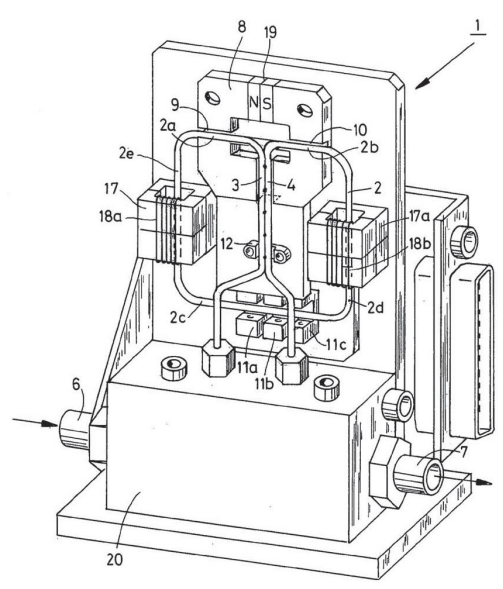

Fig. 3.1 Coriolis Mass Flow Meter [45]. The instrument is connected to a pipeline; a fluid flow enters the instrument (6), flows trough the tubewindow (2) and exits the instrument (7). The flexible tube-window (2) is actuated in resonance by an Lorentz actuator (8) and the displacements are measured by optical displacements sensors (11ac) [46].

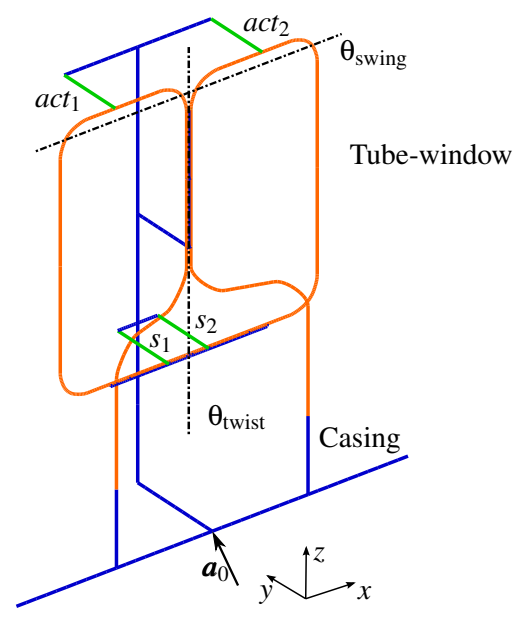

Fig. 3.2 CMFM multi-body model (Chapter 2); the flexible tube-window is actuated by two Lorentz actuators $a c t_{1}$ and $a c t_{2}$. The displacement is measured by two displacements sensors, $s_{1}$ and $s_{2}$. On the casing, a vector $\boldsymbol{a}_{0}$ with floor movements is imposed.

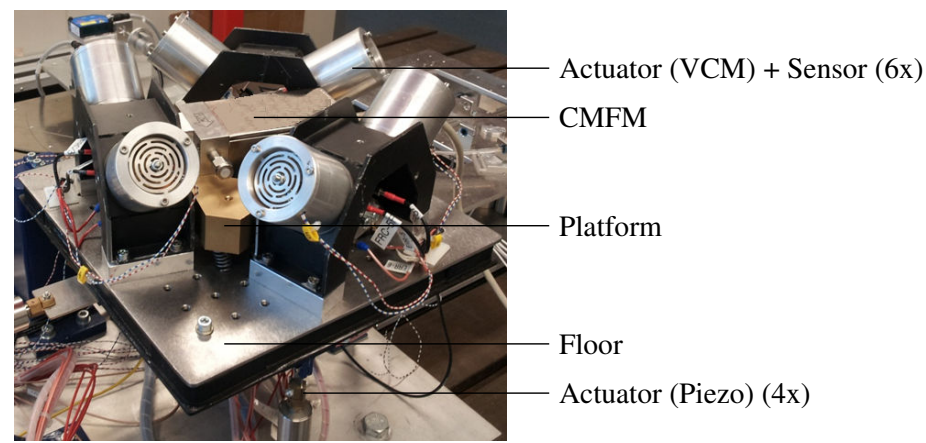

Fig. 3.3 Shaker setup - the CMFM is mounted on a Stewart platform. Voice coil actuators are used to apply forces on the low frequency $(22 \mathrm{~Hz})$ suspended platform and accelerometers are used to measure the platform vibrations $\boldsymbol{a}_{1}$ and external vibrations $\boldsymbol{a}_{0}$.

there is a mass-flow, due to the Coriolis effect. The second mode, with a natural frequency of $60 \mathrm{~Hz}$, is termed an in-plane mode, because it has no displacement in the direction of the sensors. The third mode is the actuation mode, because the tube-window is actuated to oscillate in resonance around the $\theta_{\text {twist }}$-axis at $87 \mathrm{~Hz}$. When the third mode is actuated and 
there is a mass-flow, the Coriolis mode is also actuated at the actuation frequency, due to the anti-symmetric elements of the damping matrix. This coupling occurs due to the Coriolis effect and is proportional to the mass-flow $\dot{\Phi}_{\mathrm{m}}$ and angular velocity $\dot{\theta}_{\text {twist }}$ of the tube. An expression for the Coriolis force $F_{\text {cor }}$ is given by:

$$
F_{\text {cor }}=-2 L\left(\dot{\theta}_{\text {twist }} \times \dot{\Phi}_{\mathrm{m}}\right)
$$

where $L$ is the effective length of the Coriolis tube.

The CMFM is equipped with two displacements sensors $s_{1}$ and $s_{2}$ to measure internal deformations. The differential-mode $y_{\text {act }}=1 / 2\left(s_{1}-s_{2}\right)$ is termed the actuation displacement, because it indicates a rotation around the $\theta_{\text {twist }}$-axis which is controlled actively by the Lorentz actuator ( $a c t_{1}$ and $\left.a c t_{2}\right)$. The common-mode $y_{\text {cor }}=1 / 2\left(s_{1}+s_{2}\right)$ is termed the Coriolis displacement, which indicates a rotation around the $\theta_{\text {swing }}$-axis. When there is no mass-flow, the tube window is rotating around the $\theta_{\text {twist }}$-axis, such that the phase between the signals $s_{1}$ and $s_{2}$ is equal to $180^{\circ}$. When there is a mass-flow, the phase of the sensor signals will change, because the Coriolis effect is $90^{\circ}$ out of phase with the actuation. Where the phase-difference between the two sensor signals is approximately proportional to the massflow [61]. The phase-difference between the sensor displacements $s_{1}$ and $-s_{2}$ is approximated as:

$$
\Delta \phi=\phi_{1}-\phi_{2} \approx 2 \frac{\left|s_{1}+s_{2}\right|}{\left|s_{1}-s_{2}\right|}=2 \frac{\left|y_{\text {cor }}\right|}{\left|y_{\text {act }}\right|}
$$

where the actuation amplitude $\left|y_{\text {act }}\right|$ is controlled in a feedback loop, resulting in an oscillation with a constant amplitude. The Coriolis displacment $\left|y_{\text {cor }}\right|$ is the amplitude of the sine, $90^{\circ}$ out of phase with the actuation sine. But this displacement $y_{\text {cor }}$ is not a pure sine, it is a function of the Coriolis force $F_{\text {cor }}$ and the external vibrations $\boldsymbol{a}_{0}$. In the Lapace s-domain, it is written as:

$$
y_{\mathrm{cor}}(s)=T_{y_{\mathrm{cor}}, F_{\mathrm{cor}}}(s) F_{\mathrm{cor}}(s)+\boldsymbol{T}_{y_{\mathrm{cor}, a_{0}}}(s) \boldsymbol{a}_{0}(s)
$$

The force $F_{\text {cor }}$ only occurs at the actuation frequency, see Eq. 3.1, but the external vibrations might be broadband. The transmissibility $\boldsymbol{T}_{y_{\mathrm{cor}, a_{0}}}(s)$ gives the influence of external disturbances on the Coriolis displacement. The transfer function $T_{y_{\mathrm{cor}}, F_{\mathrm{cor}}}(s)$ gives the influence of the Coriolis force $F_{\text {cor }}$ on the Coriolis displacement. Both transfer functions are obtained from the SPACAR model. To determine the amplitude of the Coriolis displacement at the actuation frequency, the signal is filtered to remove all information outside the actuation frequency band:

$$
y_{\text {cor, filt }}(s)=y_{\text {cor }}(s) F(s)
$$

where $F(s)$ is a $10 \mathrm{~Hz}$ bandpass filter around the actuation frequency $\omega_{\mathrm{act}}=87 \cdot 2 \pi \mathrm{rad} / \mathrm{s}$. To obtain the amplitude at the actuation frequency $\omega_{\text {act }}$, a frequency-shift is applied:

$$
y_{\text {cor,filt,shift }}(s)=y_{\text {cor,filt }}\left(s-\mathrm{i} \omega_{\text {act }}\right)
$$

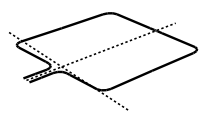


Besides a mass-flow, also external vibrations have an influence on this displacement, see Eq. 3.3. Those external vibrations are mostly broadband and can be characterised by a power spectral density $\boldsymbol{\Phi}_{a_{0}}$, which describes how the power of the signal $\boldsymbol{a}_{0}$ is distributed over the various frequencies. The influence of each frequency adds up in the cumulative RMS value, using Parseval Theorem:

$$
\sigma_{y_{\text {cor }, \text { filt }}}^{2}=\int_{-\infty}^{\infty} \boldsymbol{\Phi}_{y_{\text {cor }, \text { filt }}}(v) \mathrm{d} v=\int_{-\infty}^{\infty}\left|\boldsymbol{T}_{y_{\text {cor }, a_{0}}}(v) F(v)\right|^{2} \boldsymbol{\Phi}_{a_{0}}(v) \mathrm{d} v
$$

where the mass-flow effect, which adds up linearly, has been omitted. To estimate the influence of external vibrations on the Coriolis displacment, the filtered Coriolis displacement (Eq. 3.4) is used, because the frequency shift only changes the integration interval. Using Eq. 3.2 and 3.6 the influence of external vibrations on the phase difference can be estimated:

$$
\sigma_{\Delta \phi}=\frac{2}{\left|y_{\text {act }}\right|} \sigma_{y_{\text {cor, filt }}}=\sqrt{\int_{-\infty}^{\infty}\left|\frac{2}{\left|y_{\text {act }}\right|} \boldsymbol{T}_{y_{\mathrm{cor}, a_{0}}}(v) F(v)\right|^{2} \boldsymbol{\Phi}_{a_{0}}(v) \mathrm{d} v}
$$

Reducing the influence for a certain disturbance $\boldsymbol{\Phi}_{\boldsymbol{a}_{0}}$ can be realised by: reducing the transmissibility $\boldsymbol{T}_{y_{\text {cor }}, \boldsymbol{a}_{0}}(s)$, applying a smaller band-pass filter $F(s)$ or increasing the actuation amplitude $y_{\text {act }}$. A smaller band-pass filter has a direct impact on the response time of the CMFM and is therefore undesirable. The actuation amplitude is also limited in practice, e.g., by actuator saturation, sensor range or material strength. The transmissibility $\boldsymbol{T}_{y_{\mathrm{cor}}, \boldsymbol{a}_{0}}(s)$ can be reduced using passive or active vibration isolation. The feasibility of both is explored in this paper, whereby attenuation is needed in a band around the actuation frequency, where the band-pass filter has high gain.

In Chapter 2 it has been shown that the only relevant disturbance is a translation of the casing in the direction of the sensors, since this direction actuates the Coriolis mode directly. Therefore, only this direction is considered in this paper. Moreover, it has been shown that the essential dynamics of the response to vibrations in this direction can be modelled by a mass-spring model representing the Coriolis mode (Chapter 2). The parameters of this model are obtained from modal reduction.

\subsubsection{Vibration isolator setup}

To evaluate the possibilities for active vibration isolation, the reference instrument is mounted on a 6-DOF vibration isolation setup (Fig. 3.3). The platform is suspended at a low frequency in all directions (about $22 \mathrm{~Hz}$ ), using a Stewart type platform, mounted on a rigid floor plate. The platform is actuated by six voice coil actuators. The floor plate is equipped with four piezoelectric actuators to apply external vibrations. The vibrations of the floor and platform can be measured using accelerometers, for each direction. More details about the experimental setup can be found in Tjepkema [64]. 
Using a rigid-body model, the relation between the sensor coordinates $\boldsymbol{q}$ and the accelerations in Cartesian coordinates $\boldsymbol{x}=\left(x, y, z, \theta_{x}, \theta_{y}, \theta_{z}\right)$, based on the Cartesian coordinate system, is derived as

$$
\boldsymbol{x}_{i}=\boldsymbol{R}_{i} \boldsymbol{q}_{i}, \quad \boldsymbol{R}_{i} \in \mathbb{R}^{6 x 6}, i=0,1
$$

Vectors $\boldsymbol{q}_{0}$ and $\boldsymbol{q}_{1}$ describe the measured floor and platform motion, respectively, while $\boldsymbol{x}_{0}$ and $\boldsymbol{x}_{1}$ describe the same motions transformed to cartesian coordinates. The accelerometers on the platform are orientated in the direction of the voice coil actuators. Therefore, the matrix $\boldsymbol{R}_{1}^{-1}$ is used to apply forces in the Cartesian coordinate system.

For active vibration isolation, the setup is controlled by a dSpace digital signal processor system using a sample frequency of $6.4 \mathrm{kHz}$. Accelerometers on the platform are used to implement acceleration feedback. The measurements of the internal deformations are used to damp the Coriolis mode and to determine the mass-flow.

Power Spectral Densities (PSD) of the different discrete-time signals are estimated via Welch's method. To apply the method, the Matlab function pwelch is used. In total a dataset of $60 \mathrm{~s}$ of measurement is used. To reduce the noise level, the method uses a 24k-point symmetric Hanning window. The performance is evaluated by the transmissibility function, which is estimated using the aforementioned PSDs.

\subsubsection{Combined model for control}

In this subsection, we describe the model of the total system, i.e. when the CMFM is mounted on the vibration isolator, see Fig. 3.3. This model, shown in Fig. 3.4, is used to design the feedback controllers.

A multi-body model of a CMFM was derived in previous work and summarised in section 3.2.1 The CMFM is represented by the masses $m_{1}$ and $m_{2}$, representing the casing and the moving tube respectively. The equivalent stiffness $k_{2}$ and equivalent mass $m_{2}$ represent the Coriolis mode. The internal displacement $y_{\text {cor }}$ is measured by displacement sensors. The mounting of the CMFM onto the vibration isolator, is represented by the additional casing mass $m_{1}$ and mounting stiffness $k_{1}$. The total actuator force of the voice coil actuators is represented by $F_{\mathrm{a}}$.

In this model, the actuation mode is omitted, because this mode is orthogonal to the Coriolis mode. However, the actuation mode is coupled with the Coriolis mode by means of the Coriolis force (eq. 3.1):

$$
F_{\text {cor }} \propto \dot{y}_{\text {act }} \times \dot{\Phi}_{\mathrm{m}}
$$

This force induces a Coriolis displacment $y_{\text {cor }}$. By applying this model, the effect of external vibrations $\ddot{y}_{0}$ on $y_{\text {cor }}$ can be compared to the effect of flow $\dot{\Phi}_{\mathrm{m}}$, see Eq. 3.3

This model is used to design a controller that meets the objectives as defined in section 3.1 In terms of the model variables, these objectives can be described as follows. First the transmissibility from $\ddot{y}_{0}$ to $y_{\text {cor }}$ is reshaped using a passive suspension and active feedback (first 


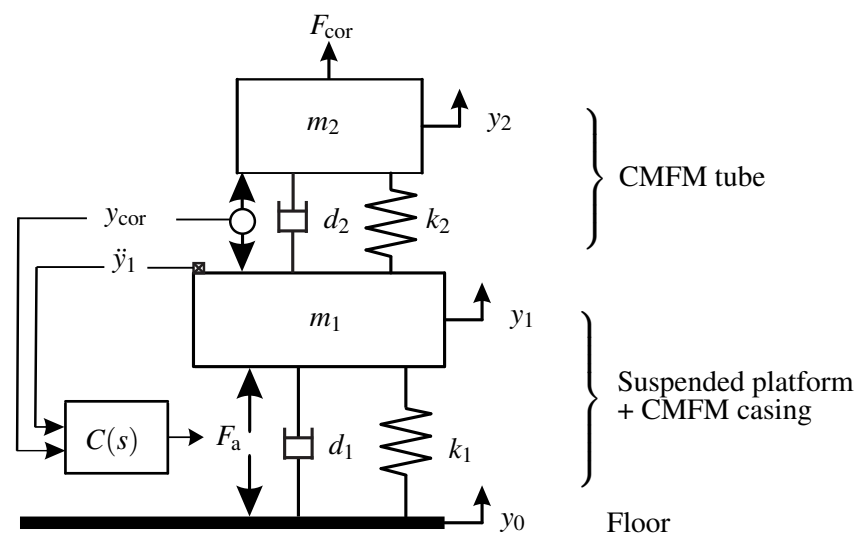

Fig. 3.4 Mass-spring model for active vibration isolation. External vibrations are denoted by $y_{0}$. The mass of the suspended platform including the CMFM casing is represented by $m_{1}$ and the suspension of the platform is formed by spring $k_{1}$. The Coriolis mode of the CMFM tube is represented by $m_{2}$ and $k_{2}$. Sensors are placed to measure $\ddot{y}_{1}$ and $y_{\mathrm{cor}}=y_{2}-y_{1}$. Force $F_{\mathrm{a}}$ represents the actuator.

and second objective):

$$
T_{Y_{\mathrm{cor}}, \ddot{Y}_{0}}(s)=\frac{Y_{\mathrm{cor}}(s)}{\ddot{Y}_{0}(s)}
$$

Second, no interference with the flow measurement (third objective) is realised by leaving the following transfer function unchanged:

$$
G_{Y_{\mathrm{cor}}, F_{\mathrm{cor}}}(s)=\frac{Y_{\mathrm{cor}}(s)}{F_{\mathrm{cor}}(s)}
$$

In this study, three different configurations are considered: (1) a reference model configuration, whereby the CMFM is connected rigidly to the floor; (2) the case, whereby the CMFM is passively suspended on the vibration isolator; and (3) the case in which active vibration isolation is added to the passively suspended CMFM. The passive and active model have the same physical parameters, as given in Table 3.1 . Notice the ratio between the masses $m_{1}$ and $m_{2}$; the mass of the Coriolis tube is small compared to that of the casing mass.

Table 3.1 Model parameters for all presented configurations of mass-spring model given in Fig. 3.4

\begin{tabular}{lcccccc}
\hline Model & $\begin{array}{c}m_{1} \\
(\mathrm{~kg})\end{array}$ & $\begin{array}{c}d_{1} \\
(\mathrm{~N} \mathrm{~s} / \mathrm{m})\end{array}$ & $\begin{array}{c}k_{1} \\
(\mathrm{~N} / \mathrm{m})\end{array}$ & $\begin{array}{c}m_{2} \\
(\mathrm{~kg})\end{array}$ & $\begin{array}{c}d_{2} \\
(\mathrm{~N} \mathrm{~s} / \mathrm{m})\end{array}$ & $\begin{array}{c}k_{2} \\
(\mathrm{~N} / \mathrm{m})\end{array}$ \\
\hline Reference & 1.2 & $\infty$ & $\infty$ & $5.2 \times 10^{-5}$ & $9 \times 10^{-6}$ & 3.6 \\
Passive & 6.5 & 20 & $1.25 \times 10^{5}$ & $5.2 \times 10^{-5}$ & $9 \times 10^{-6}$ & 3.6 \\
Active & 6.5 & 20 & $1.25 \times 10^{5}$ & $5.2 \times 10^{-5}$ & $9 \times 10^{-6}$ & 3.6 \\
\hline
\end{tabular}

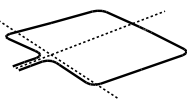




\subsection{Control design}

In this section, it is explained how active vibration isolation control can be used to improve the performance of a CMFM. In the first subsection, a feedback controller design is proposed that fulfils the first performance criterion. Second, an additional controller is presented to damp the internal mode, fulfilling the second and third criteria. In the final subsection the performance of the designed controllers is discussed.

\subsubsection{Reducing the transmissibility}

Reconsider the model from Fig. 3.4 We will use acceleration feedback, by measuring $\ddot{y}_{1}$, to reduce the transmissibility of external vibrations to the platform. The following system has to be controlled:

$$
\begin{aligned}
G_{\mathrm{pl}}(s) & =\frac{\ddot{Y}_{1}(s)}{F_{\mathrm{a}}(s)}=\frac{1}{m_{1}} \frac{s^{2}\left(s^{2}+2 \zeta \omega_{\mathrm{ar}} s+\omega_{\mathrm{ar}}^{2}\right)}{\left(s^{2}+2 \zeta \omega_{1} s+\omega_{1}^{2}\right)\left(s^{2}+2 \zeta \omega_{2} s+\omega_{2}^{2}\right)} \\
& \approx \frac{1}{m_{1}} \frac{s^{2}}{s^{2}+2 \zeta \omega_{1} s+\omega_{1}^{2}}
\end{aligned}
$$

Where $\omega_{1}$ and $\omega_{2}$ are the resonance frequencies and $\omega_{\mathrm{ar}}$ is the anti-resonance frequency of the mass-spring system of Fig. 3.4 The approximation in Eq. 3.13 is valid because $m_{2} \ll m_{1}$, see Table 1 . In that case, a pole/zero cancellation occurs because $\omega_{2} \approx \omega_{\mathrm{ar}}=\sqrt{k_{2} / m_{2}}$, while $\omega_{1} \approx \sqrt{k_{1} / m_{1}}$.

In van Dijk [77] a method is described to reduce the transmissibility of external vibrations using feedback control. In this method, virtual mass and virtual skyhook damping are added to $m_{1}$. The effect of adding virtual mass is that the suspension frequency is actively reduced, resulting in more vibration isolation in the region of interest. A virtual skyhook damper adds damping to the suspension mode without affecting the vibration isolation at high frequencies [34]. A PI controller is used to add virtual mass by the proportional (P) action and virtual skyhook damping by the integrating (I) action [77]:

$$
C_{1}(s)=\frac{F_{\mathrm{a}}(s)}{\ddot{Y}_{1}(s)}=-\left(K_{\mathrm{a}}+\frac{K_{\mathrm{v}}}{s}\right)
$$

According to van Dijk [77], $K_{\mathrm{a}}$ and $K_{\mathrm{v}}$ are calculated as follows:

$$
\begin{aligned}
& K_{\mathrm{a}}=m_{1}\left(\frac{f_{\text {susp }}^{2}}{f_{\mathrm{n}}^{2}}-1\right) \\
& K_{\mathrm{v}}=2 \zeta_{\mathrm{n}} m_{1} \frac{f_{\text {susp }}}{f_{\mathrm{n}}}\left(2 \pi f_{\text {susp }}\right)
\end{aligned}
$$

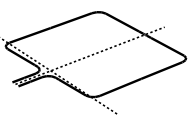


In these equations, $f_{\mathrm{n}}$ is the desired closed loop suspension frequency with relative damping $\zeta_{\mathrm{n}}$ and $f_{\text {susp }}=\omega_{1} / 2 \pi=22 \mathrm{~Hz}$ is the passive suspension frequency of the platform. The lower the value of $f_{\mathrm{n}}$, the greater the isolation. The achievable $f_{\mathrm{n}}$ is limited to $14 \mathrm{~Hz}$, due to closed loop stability limitations of the experimental setup. Therefore, we set $f_{\mathrm{n}}=14 \mathrm{~Hz}$ with $70 \%$ skyhook damping $\left(\zeta_{\mathrm{n}}=0.7\right)$. The corresponding controller gains for $C_{1}(s)$ are $K_{\mathrm{a}}=9.6$ and $K_{\mathrm{v}}=1977$. The attenuation at the actuation frequency of the CMFM is approximated by:

$$
T_{\ddot{Y}_{1}, \ddot{Y}_{0}}(s) \approx \frac{\omega_{1}^{2}}{s^{2}+\omega_{1}^{2}} \rightarrow \mid \text { attenuation } \mid=\frac{\omega_{1}^{2}}{\omega_{\text {act }}^{2}} \quad\left(\omega_{\text {act }}^{2}>>\omega_{1}^{2}\right)
$$

This results in an attenuation at the actuation frequency of $24 \mathrm{~dB}$ for the passive case with $f_{\text {susp }}=22 \mathrm{~Hz}$. Adding active vibration control, the attenuation becomes $32 \mathrm{~dB}$, as shown in Fig. 3.6. In the region of interest, the improvement is only $8 \mathrm{~dB}$ with respect to passive isolation. The figure also shows that this controller has an infinite bandwidth, because the plot of the active system is under the plot of the passive system at all frequencies. Therefore, this controller cannot be implemented in practice.

To improve the performance of the active system, three loop shaping filters are added to $C_{1}(s)$. This results in an expression for controller $C_{2}(s)$ :

$$
C_{2}(s)=\frac{F_{\mathrm{a}}(s)}{\ddot{Y}_{1}(s)}=C_{1}(s) \underbrace{\frac{s^{2}}{s^{2}+2 \zeta_{\mathrm{f}} \omega_{f} s+\omega_{\mathrm{f}}^{2}}}_{H_{1}(s)} \underbrace{\frac{\omega_{\mathrm{r}}^{2}}{s^{2}+2 \zeta_{\mathrm{r}} \omega_{r} s+\omega_{\mathrm{r}}^{2}}}_{H_{2}(s)} \underbrace{\frac{s+\omega_{\mathrm{Z}}}{\omega_{\mathrm{Z}}}}_{H_{3}(s)}
$$

The term $H_{1}(s)$ is a second-order highpass filter at $\omega_{\mathrm{f}}=1 \cdot 2 \pi \mathrm{rad} / \mathrm{s}$ with $\zeta_{\mathrm{f}}=0.7$. This filter is used to cut off the integrating action at low frequencies and to prevent actuator saturation. The term $H_{2}(s)$ is a second-order low-pass filter at $\omega_{\mathrm{r}}=87 \cdot 2 \pi \mathrm{rad} / \mathrm{s}$ which is equal to the actuation frequency of the CMFM tube. This low-pass filter limits the open-loop controller bandwidth, which is necessary to prevent instability due to higher-order dynamics. The relative damping of $H_{2}(s)$ is set sufficiently low $\left(\zeta_{\mathrm{r}}=0.07\right)$ such that a resonance peak appears in the controller at $87 \mathrm{~Hz}$. The resonance peak strongly increases the controller action -and thus the vibration isolation- in a narrow frequency band around $\omega_{\mathrm{r}}$. In our case, this narrow frequency band is designed such that vibration isolation is increased in the region of interest, adding $\frac{1}{2 \zeta_{\mathrm{r}}}=17$ $\mathrm{dB}$ of extra attenuation. The term $H_{3}(s)$ is a zero at $\omega_{\mathrm{z}}=140 \cdot 2 \pi \mathrm{rad} / \mathrm{s}$. For stability reasons this zero is added to increase the phase margin at the high cross-over frequency, which is the frequency where the loop gain $G_{\mathrm{pl}}(s) C_{2}(s)$ is equal to 1 . Bode plots of the controllers $C_{1}$ and $C_{2}$ are depicted in Fig. 3.5 .

The vibration isolation performance of the active system using $C_{2}(s)$ is also shown in Fig. 3.6. Compared with $C_{1}(s)$, controller $C_{2}(s)$ provides more vibration isolation in the region of interest because of the poorly damped resonance filter in $H_{2}(s)$. Furthermore, it is observed that the bandwidth is limited to $140 \mathrm{~Hz}$, because at this frequency the performance of the active system equals that of the passive system. Therefore, controller $C_{2}(s)$ performs better then $C_{1}(s)$. 


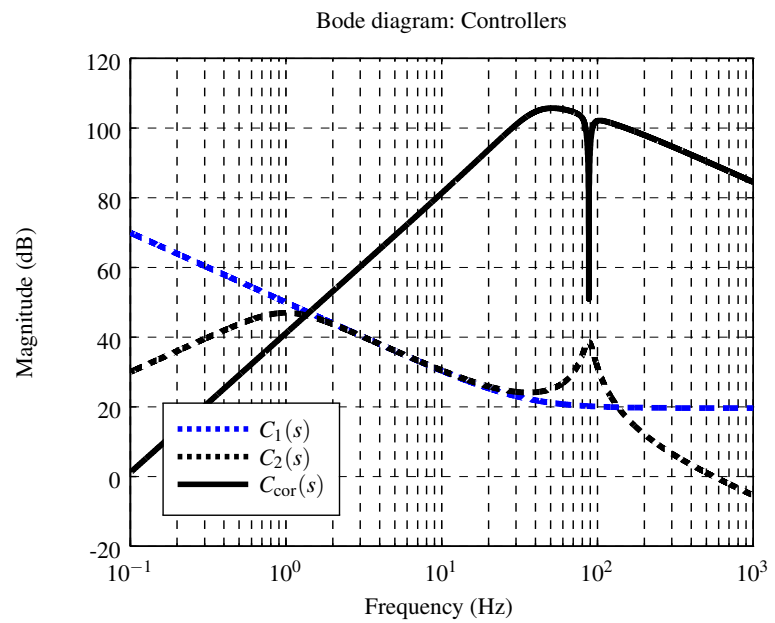

Fig. 3.5 Bode magnitude plot, showing the three different controllers

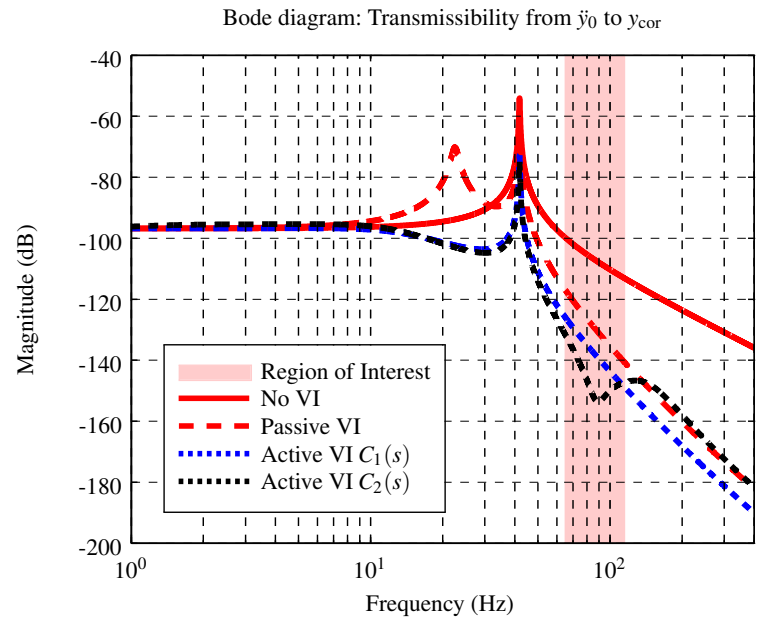

Fig. 3.6 Bode magnitude plot, showing the vibration isolation (VI) transmissibility in the case of no VI, passive VI and active VI. The focus for VI is on the region of interest, because the CMFM uses this frequency band to measure the mass flow, see Eq. 3.5

\subsubsection{Damping the Coriolis mode}

Controller $C_{2}(s)$ increases the vibration isolation in the region of interest. However, the $39 \mathrm{~Hz}$ Coriolis mode of the tube is still undamped. See Fig. 3.6, the internal mode is invisible for the acceleration sensors because of a pole/zero cancellation, zie Eq. 3.13, because $m_{1} \gg m_{2}$. Although the Coriolis mode is outside the region of interest, it must be damped to prevent

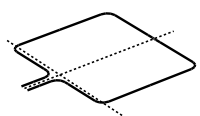


that the tube moves out of the sensor range. Moreover, the definition of the region of interest is not very strict, so a high peak just outside this region will still increase the sensitivity for external vibrations.. Therefore, a second controller $C_{\text {cor }}(s)$ is added to the system that damps the Coriolis mode of the tube. This is achieved by measuring $y_{\text {cor }}$ and feeding this displacement back to $C_{\text {cor }}(s)$. The output of $C_{\text {cor }}(s)$ is provided to the same actuators as used for $C_{2}(s)$. The block scheme in Fig. 3.7 shows the resulting controller structure. In this figure, $G_{\mathrm{pl}}(s)$ is given by Eq. (3.12), and $G_{\mathrm{cor}}(s)$ is given by:

$$
G_{\mathrm{cor}}(s)=\frac{Y_{\mathrm{cor}}(s)}{F_{\mathrm{a}}(s)}=\frac{-s^{2}}{m_{1}\left(s^{2}+2 \zeta \omega_{1} s+\omega_{1}^{2}\right)\left(s^{2}+2 \zeta \omega_{2} s+\omega_{2}^{2}\right)}
$$

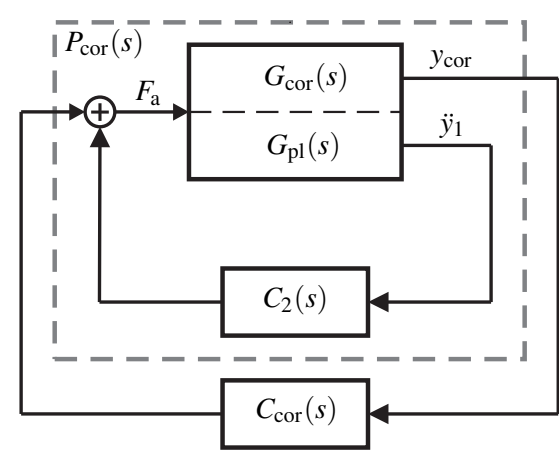

Fig. 3.7 Structure of the combined control loop, formed by the Coriolis mode damping controller $C_{\text {cor }}(s)$ in combination with the previously designed controller $C_{2}(s)$ for reducing the transmissibility.

Before $C_{\text {cor }}(s)$ is designed, first the control loop for controller $C_{2}(s)$ is closed. The closed loop system including $C_{2}(s)$ is then considered as a new "open loop" plant $P_{\text {cor }}(s)$ (see Fig. 3.7), for which $C_{\text {cor }}(s)$ is designed. $P_{\text {cor }}(s)$ has the following expression:

$$
P_{\text {cor }}(s)=\frac{1}{1+C_{2}(s) G_{\mathrm{pl}}(s)} G_{\mathrm{cor}}(s)
$$

Conventionally, just a differentiating action (D-action) would be used in $C_{\text {cor }}(s)$ to damp the Coriolis mode in $P_{\text {cor }}(s)$. Such a D-action has a phase angle of $+90^{\circ}$. However, the D-action is only necessary in the frequency range around the Coriolis mode. At low frequencies, the controller gain must be low to prevent stability problems due to the suspension mode. To realize a controller behaving like a $\mathrm{D}$-action around the Coriolis mode, a second-order highpass filter for $C_{\text {cor }}(s)$ is used The filter frequency is $\omega_{1}=39 \cdot 2 \pi \mathrm{rad} / \mathrm{s}$ and relative damping $\zeta_{1}=0.5$. This type of controller is also used in positive position feedback [52], which is used to damp a specific mode in a system. The filter frequency is set equal to the Coriolis frequency of the tube, such that at $\omega_{1}$ the phase angle is $+90^{\circ}$. This high-pass filter limits the controller action at low frequencies. At high frequencies, the controller gain must be small to prevent stability problems caused by unmodeled higher-order dynamics, therefore a single pole is

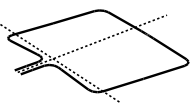


added at $2 \omega_{1}$. Furthermore, $C_{\text {cor }}(s)$ may not influence the actuation mode at $87 \mathrm{~Hz}$, because then the flow-induced Coriolis displacement at $87 \mathrm{~Hz}$, which is used to measure the mass flow, is reduced by this controller. To ensure that absolutely no control action is performed at the actuation frequency (Third objective), a notch filter will be applied at the actuation frequency $(87 \mathrm{~Hz})$. Omitting the notch filter results in an offset in the measurement value. The notch filter frequency is made adaptive, so that it moves with the actuation frequency, which is dependent on the density of the medium flowing through the tube. This leads to the following design for $C_{\text {cor }}(s)$ :

$$
C_{\text {cor }}(s)=\frac{F_{\mathrm{a}}(s)}{Y_{\text {cor }}(s)}=k_{\mathrm{p}} \frac{s^{2}}{\left(s^{2}+2 \zeta_{1} \omega_{1} s+\omega_{1}^{2}\right)} \frac{2 \omega_{1}}{s+2 \omega_{1}} N(s)
$$

The controller gain $k_{\mathrm{p}}=200 \cdot 10^{3}$ is tuned such that the Coriolis mode is damped without making the closed loop system unstable. The term $N(s)$ represents the adaptive notch filter. The controller is depicted in Fig. 3.5

\subsubsection{Control performance}

The modelled transmissibility of the active system with the combined control loop is shown in Fig. 3.8. Compared to the active system using only $C_{2}(s)$, the combined control loop damps the resonance peak of the Coriolis mode with $40 \mathrm{~dB}$, while the performance is not negatively affected in the region of interest.

The combination of $C_{2}(s)$ and $C_{\text {cor }}(s)$ applied to Coriolis Mass-Flow meters results in a large attenuation of $49 \mathrm{~dB}$ at the actuation frequency, without affecting the sensitivity for flow measurements. Therefore, the control algorithm satisfies the stated objectives and will be validated in the next section.

\subsection{Experimental results}

In this section, the model results are validated using the experimental setup, which was described in section 3.2.2. First, the influence of external vibrations on a Coriolis displacement is validated. Second, the effect of vibration isolation on a mass-flow measurement with and without a mass-flow is shown.

\subsubsection{Model validation}

The frequency response from floor acceleration $\ddot{y}_{0}$ to Coriolis displacement $y_{\text {cor }}$ is measured to validate the transmissibilities from Fig. 3.8, using the setup described in section 3.2.2.

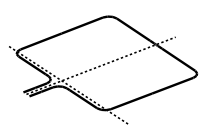




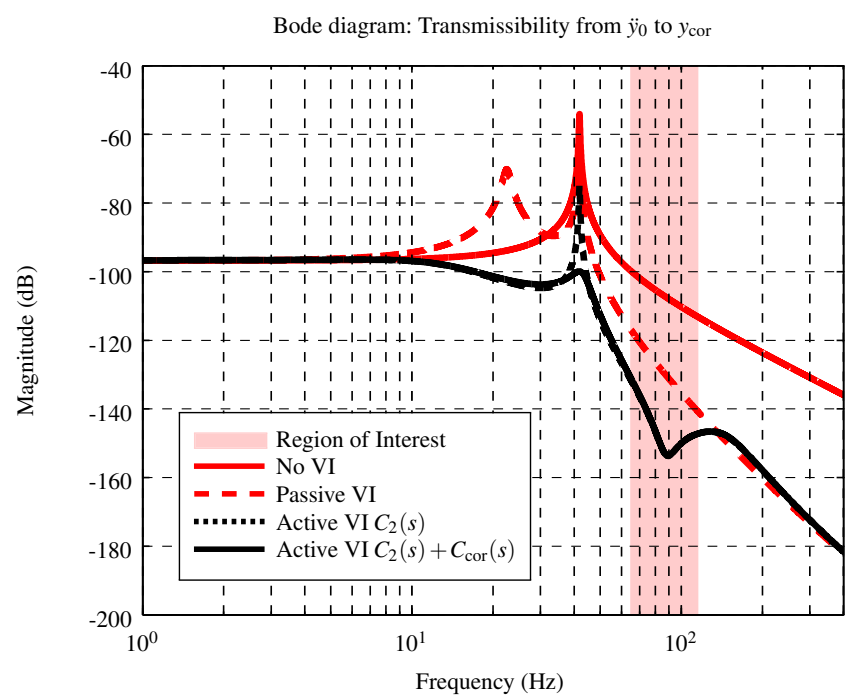

Fig. 3.8 Compared to the previously designed active vibration isolation system using $C_{2}(s)$, the combined control loop also damps the Coriolis mode in the transmissibility.

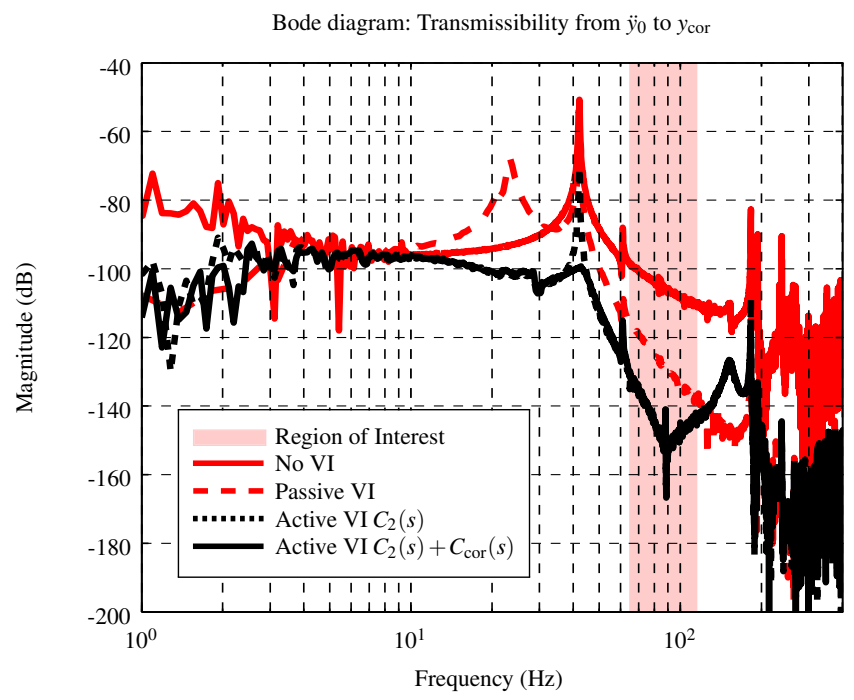

Fig. 3.9 Experimental validation of the modelled transmissibilities shown in Fig. 3.8

The experimental results are shown in Fig. 3.9. The measurements show a good agreement with the modelled results in Fig. 3.8 for the mid-frequency range. At low and high frequencies $(<5 \mathrm{~Hz}$ and $>200 \mathrm{~Hz})$, the measurements become noisy because the Coriolis displacement is 
too low to be measured by the optical sensors and at these frequencies there is no disturbance provided to the floor plate. When only passive vibration isolation is used, the poorly damped suspension mode of the platform at $22 \mathrm{~Hz}$ is clearly visible. Compared to the non-isolated case, passive vibration isolation adds $20 \mathrm{~dB}$ attenuation in the area of interest. In the case of active vibration isolation with controller $C_{2}(s)$, the suspension mode is damped and reduced to $14 \mathrm{~Hz}$. Active vibration isolation results in $46 \mathrm{~dB}$ attenuation of vibrations around the actuation frequency, compared to the non-isolated case. Using the combined control loop, the resonance peak of the Coriolis mode at $42 \mathrm{~Hz}$ has been dropped by $40 \mathrm{~dB}$, while keeping the $46 \mathrm{~dB}$ attenuation in the region of interest.

A large difference is the resonance frequency at $188 \mathrm{~Hz}$. This is a higher order mode of the system, which is not included in the mass-spring model (Fig. 3.4). Small differences between the modelled and measured transmissibility are also visible. In the case of active vibration isolation, an additional peak shows up at $150 \mathrm{~Hz}$. This peak is caused by the induction poles of the voice coil actuators, which decrease the phase margin at the cross-over frequency. At $30 \mathrm{~Hz}$, a platform suspension mode in another direction is visible as a dip in the transfer function. At $60 \mathrm{~Hz}$, a pole/zero cancellation shows up, which is caused by the in-plane mode of the flow tube. At the actuation mode at $87 \mathrm{~Hz}$, a pole/zero cancellation also occurs because of a small misalignment of the optic sensors. Neither of these differences are expected to affect the performance significantly. This will be shown in the next subsection.

\subsubsection{Influence of external vibrations on the mass-flow measurement}

In this subsection, the impact of reducing the transmissibility $T_{y_{\mathrm{cor}}, \ddot{y}_{0}}$ on the measurement value $\Delta \phi$ is shown. For a broadband external disturbance, the influence of each frequency adds up in the cumulative RMS measurement value $\sigma_{\Delta \phi}$ of a CMFM measurement; see Eq. 3.6. In Fig. 3.10, the Power Spectral Density (PSD) of several disturbance levels for the performed experiments are given. To indicate the level of those disturbances, Vibration Criterion (VC) curves [23] are added. The VC-curves are meant as upper bounds for the peaks in the floor vibration spectrum. For disturbance 1, only the background vibration level of the setup is used. For disturbance levels 2 and 3, the input is shaped to achieve a flat disturbance between 10 and $100 \mathrm{~Hz}$ with PSD levels of $10^{-5}$ and $10^{-4}\left(\mathrm{~m} / \mathrm{s}^{2}\right)^{2} / \mathrm{Hz}$ respectively, provided by the piezo shakers of the setup. Those levels are chosen for easy comparison of different CMFM instruments and the bandwidth is chosen such that it contains both the Coriolis and actuation frequency. The resulting RMS error is given in Table 3.2

The transmissibility $T_{y_{\mathrm{cor}}, \ddot{y}_{0}}$ is different for each of the four different configurations (reference, passive and active with and without Coriolis mode damping), and the model claims an attenuation of $24 \mathrm{~dB}$ and $49 \mathrm{~dB}$ for the passive and active configuration respectively by the same disturbance input for frequencies around the actuation frequency, see Fig. 3.8. In Table 3.2 is shown that for disturbance 1: the influence reduces for the passive and active configurations with $8.8 \mathrm{~dB}$ with respect to the reference configuration. The reduction is limited, because the value, $0.36 \mathrm{rad}$, is the lower limit due to the measurement noise of the displacement sensors, independent of external vibrations. For disturbance 2, the influence re-

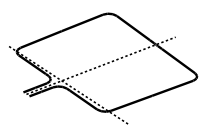




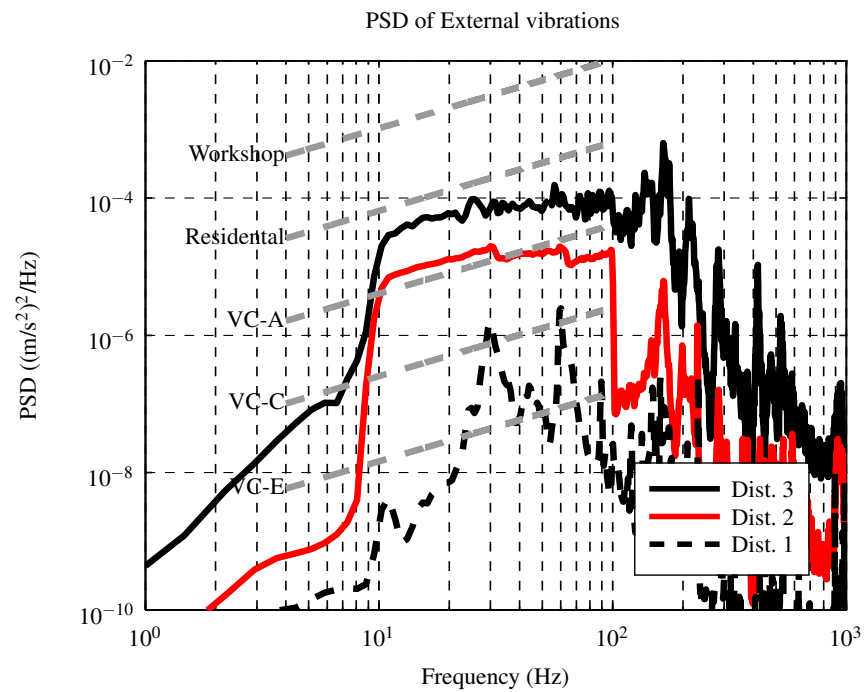

Fig. 3.10 Applied disturbances $\ddot{y}_{0}$ of the three levels, compared to vibration criterion (VC) curves [23]

Table 3.2 Experimental RMS flow error results of disturbances given in Fig. 3.10 with $10 \mathrm{~Hz}$ filter. Values are normalised by the first value.

\begin{tabular}{|c|c|c|c|c|c|c|c|c|c|}
\hline \multirow[t]{2}{*}{ Disturbance } & \multirow{2}{*}{$\begin{array}{c}\text { Flow } \\
\%\end{array}$} & \multicolumn{2}{|c|}{ Reference } & \multicolumn{2}{|c|}{ Passive } & \multicolumn{2}{|c|}{$\begin{array}{l}\text { Active } \\
C_{2}(s)\end{array}$} & \multicolumn{2}{|c|}{$\begin{array}{c}\text { Active } \\
C_{2}(s)+C_{\text {cor }}(s)\end{array}$} \\
\hline & & (rad) & $(\mathrm{dB})$ & (rad) & $(\mathrm{dB})$ & $(\mathrm{rad})$ & $(\mathrm{dB})$ & (rad) & $(\mathrm{dB})$ \\
\hline \multirow[t]{3}{*}{1} & 0 & 1.000 & 0 & 0.3762 & -8.49 & 0.3618 & -8.83 & 0.3607 & -8.86 \\
\hline & 30 & 1.125 & 0 & 0.3696 & -9.67 & 0.3629 & -9.83 & 0.3441 & -10.29 \\
\hline & 100 & 0.966 & 0 & 0.3940 & -7.79 & 0.3596 & -8.58 & 0.3585 & -8.61 \\
\hline \multirow[t]{3}{*}{2} & 0 & 17.43 & 0 & 1.497 & -21.32 & 0.4018 & -32.75 & 0.3851 & -33.11 \\
\hline & 30 & 18.34 & 0 & 1.528 & -21.59 & 0.4129 & -32.95 & 0.3929 & -33.38 \\
\hline & 100 & 17.91 & 0 & 1.544 & -21.29 & 0.4029 & -32.96 & 0.3973 & -33.08 \\
\hline \multirow[t]{3}{*}{3} & 0 & 45.64 & 0 & 3.638 & -21.97 & 0.5549 & -38.30 & 0.4606 & -39.92 \\
\hline & 30 & 45.45 & 0 & 3.852 & -21.44 & 0.5838 & -37.83 & 0.4706 & -39.70 \\
\hline & 100 & 46.73 & 0 & 3.721 & -21.98 & 0.5760 & -38.18 & 0.4573 & -40.19 \\
\hline
\end{tabular}

duces for the passive case with $21 \mathrm{~dB}$ and with active vibration isolation with $33 \mathrm{~dB}$. For the passive configuration, the reduction is as expected, but for the active case the reduction is still limited, because of the lower limit due to measurement noise. For disturbance 3, the influence reduces for both configurations with 22 and $38 \mathrm{~dB}$. The result is close to the expected values from the validation described in section 3.4.1

Adding extra damping to the Coriolis mode to prevent non-linear behaviour due to large tube-displacements is only expected to be effective for large external vibrations. Disturbance 3 is not large enough to demonstrate this effect. Due to limitations in the setup, a higher disturbance level (see Fig. 3.10) is unachievable. Therefore, the effect of the second objective 
cannot be proved for this controller design, but there is an attenuation gain of $2 \mathrm{~dB}$ achieved by damping the Coriolis mode.

The third objective requires that vibration isolation does not affect the mass-flow measurement. Therefore the RMS noise levels are compared for the various disturbance levels when a fluid (air) is also flowing through the CMFM. The results for a 0, 30 and $100 \%$ flow level are also given in Table 3.2. A $100 \%$ flow is a flow resulting in a 1 bar pressure drop over the CMFM. Comparing all the data, it is concluded that vibration isolation has no significant influence on the mean value of the flow measurement. Furthermore, it can be concluded that there is a minimal influence on the RMS level of the flow measurement value. Therefore, the active vibration isolation does not affect proper mass-flow measurements of the CMFM.

\subsection{Discussion}

In this section, the significance of the results of the presented work is discussed. First, the limitations of the feedback control system are discussed. Second, the implementation of active vibration isolation into a commercial CMFM design is considered.

\subsubsection{Limitations}

The presented values are dependent on the tube-window design and filter characteristics of the reference instrument used, but they give a realistic indication of the achievable performance. Compared to the reference system, the broadband external disturbances can be increased by $40 \mathrm{~dB}$ before they influence the measurement value of the actively suspended CMFM.

The minimum noise level of the measurement value is dependent on the noise levels of the tube displacement sensors. The minimum measurement noise level cannot be reduced by active vibration isolation, but only by improving the specifications of the tube displacement sensors, which is beyond the scope of this study.

Based on the transmissibility function, the attenuation at the actuation frequency is $49 \mathrm{~dB}$, but the reduction in the measurement value is only $40 \mathrm{~dB}$ as a result of the width of the measurement filter. The transmissibility function needs to be lowered over a wider bandwidth (e.g. by adjusting the second-order low-pass filter in $C_{2}(s)$ (Eq.3.18)). Furthermore, the performance is limited due to the limited bandwidth of the feedback control, which is due to the higher-order dynamics of the experimental setup. Therefore, the suspension frequency cannot be reduced to values lower than $14 \mathrm{~Hz}$. However, by improving the experimental setup, the performance can be improved.

The presented control algorithm design is only one-directional. Although external disturbances can occur in all translational and rotational directions, this is no limitation, because in Chapter 2.5.1 it is concluded that there is only one dominant direction in which a translation disturbance has an effect on the mass-flow measurement value. This means that the sensitivity for external vibrations, occurring in all directions, can be sufficiently reduced by applying

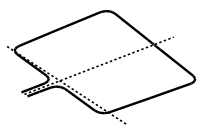


active vibration isolation in only one direction. However, a limitation of this approach is that the acceleration measurement orientation needs to be precise in the dominant direction. Also a passively isolated design can perform worse than the reference design, because of the increased gain around the suspension frequency in the transmissibility function.

\subsubsection{Implementation}

Using the shaker setup (Fig. 3.3, including a reference CMFM, allowed fast validation of the approach, but increases the total cost, size and the weight of a flow measurement instrument enormously. With only one active direction, the number of actuators and sensors can be reduced and the dimensions of the platform can be minimised. It is yet unknown whether any scaling effects will occur when reducing the size. For example, with the current mass-ratio of the tube and platform, the internal tube-window actuator and a fluid flow have a minimal effect on the platform displacement. However, this will not necessarily be the case for a miniaturized setup, and will be subject of future research.

\subsection{Conclusions}

An active vibration isolation strategy for reducing the influence of external vibrations on a Coriolis Mass Flow Meter (CMFM) measurement was presented. The active vibration isolation control results in $40 \mathrm{~dB}$ reduction of the influence of external vibrations, without affecting the mass-flow measurement.

A suspension was added to the CMFM to obtain passive isolation, attenuating the effect of high-frequency disturbances. Parasitic stiffness and high internal stresses of the fluidconveying connection-tubes limit the passive suspension frequency and thus the passive vibration isolation performance. The transmissibility of external vibrations is reduced further actively in the region of interest by acceleration feedback. A PI-controller is implemented to lower the suspension frequency and to add skyhook damping. Furthermore, the poorly damped internal mode is damped using positive position feedback of the internal deformation. These known vibration isolation strategies are adapted and tuned for the CMFM. The roll-off of the acceleration feedback is tuned such that additional suppression is obtained in the region of interest. Furthermore a notch filter is included in the positive position feedback loop to remove its effect on the flow measurement.

The model results are validated by mounting a CMFM on a six degrees-of-freedom platform with six actuators and accelerometers, thus creating an active suspension mode. Experimental results agree with the modelled results, both (1) for the transmissibility of external vibrations to the internal deformation and (2) broadband disturbances on the CMFM measurement value. The integrated mechanical design of the instrument with active vibration isolation will be a subject of future research. 


\title{
Chapter 4 \\ CMFM with integrated Active Vibration Isolation Control
}

\begin{abstract}
Vibration isolation of more than $40 \mathrm{~dB}$ is achieved for a Coriolis Mass-Flow Meter (CMFM) with integrated Active Vibration Isolation. A CMFM is an active device based on the Coriolis force principle for direct mass-flow measurements independent of fluid properties. The mass-flow measurement is derived from tube displacement measurements. Support excitations can introduce motions that cannot be distinguished from the Coriolis force induced motion, thus introducing a measurement error. Therefore, the measurement stage is passively suspended at $30 \mathrm{~Hz}$ in the 3 out-of-plane directions. Active vibration isolation is added to increase the attenuation.

In this paper the system model and controller design are presented. Based on the model an on-scale proof of principle is built and the model and controller are validated in multi-DOF. Acceleration feedback and a novel adaptive feedforward control strategy are compared A filtered-reference least-mean-square (FxLMS) adaptive scheme is used to determine the optimal feedforward controller parameters to minimise a squared error signal; the motion of the measurement stage. Both strategies result in an attenuation of 10 to $20 \mathrm{~dB}$ at $175 \mathrm{~Hz}$ in addition to the $30 \mathrm{~dB}$ attenuation obtained by the $30 \mathrm{~Hz}$ passive vibration isolation stage. The performance of the feedback strategy is limited by robust stability and the the feedforward performance is limited by sensor noise.
\end{abstract}

This chapter is preprinted from: L. van de Ridder, W. B. J. Hakvoort, D. M. Brouwer, J. van Dijk, J. C. Lötters, and A. de Boer. Coriolis mass-flow meter with integrated multi-dof active vibration isolation. 2015. Submitted to Mechatronics (Elsevier)

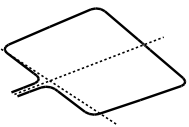




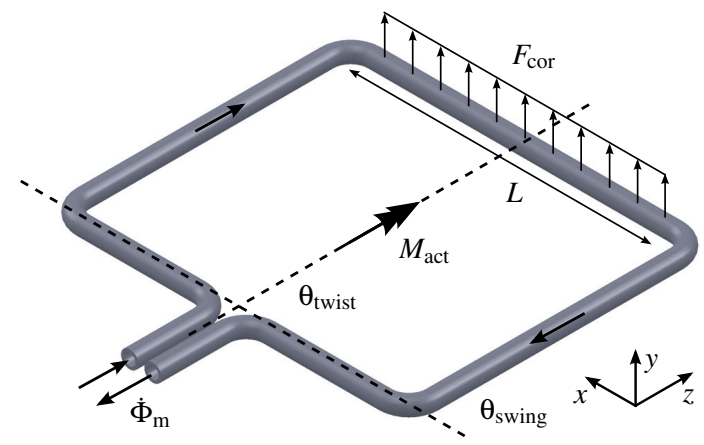

Fig. 4.1 Simplified model of a window-shaped fluid-conveying tube to show the main Coriolis force contribution. The tube is actuated to resonate around the $\theta_{\text {twist }}$-axis. Due to the Coriolis effect the tube also oscillates around the $\theta_{\text {swing }}$-axis, proportional to the mass-flow $\dot{\Phi}_{\mathrm{m}}$.

\subsection{Introduction}

A Coriolis Mass-Flow Meter (CMFM) is an active device based on the Coriolis force principle for direct mass-flow measurements independent of fluid properties [1, 78]. The CMFM contains a fluid-conveying tube. An example of a window-shaped tube is depicted in Fig.4.1 The tube is actuated to oscillate in resonance with a low amplitude around the $\theta_{\text {twist }}$-axis by the moment $M_{\text {act }}$. A fluid flow in the vibrating tube induces Coriolis forces, proportional to the mass-flow $\dot{\Phi}_{\mathrm{m}}$ :

$$
F_{\text {cor }}=-2 L \cdot \dot{\theta}_{\text {twist }} \times \dot{\Phi}_{\mathrm{m}}
$$

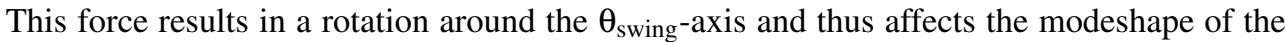
actuation mode. The rotation around the swing-axis can be derived from the tube displacement measurements and is directly proportional to the mass-flow. The motions induced by the Coriolis force are in the order of nanometres for micrometre actuation displacement. Support excitations can introduce motions that cannot be distinguished from the Coriolis force induced motion, thus introducing a measurement error [14, 72].

To reduce the sensitivity of the flow measurement to external vibrations, passive or active vibration isolation can be used. Passive isolation consists of one or multiple stages of massspring-damper systems between the floor and the frame of a machine [53]. The parameters are adjusted to achieve sufficient attenuation at certain frequency domains. However, the performance of passive isolation applied to a CMFM is limited (see Chapter 3). An alternative and widely used approach is to apply active vibration isolation control (AVIC). For recent examples of active vibration isolation for precision equipment, see [10, 64, 42, 43].

In this paper we investigate self-tuning feedforward control, as introduced in [4], and compare it to a feedback strategy [77, 52], both applied to a CMFM. These strategies are compared on the ability to reduce the influence of external vibrations on the mass-flow measurement value. Several advances are made on previous work [70, 74, 59]: (i) the feedforward strat- 


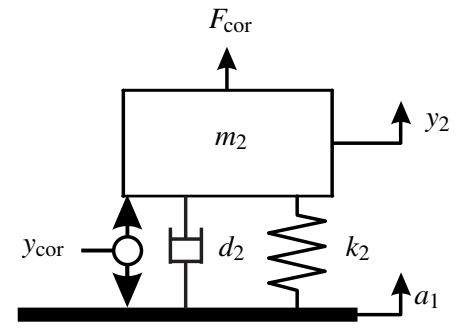

Fig. 4.2 Simplified 1D model of the CMFM tube, representing the Coriolis mode (Chapter2.

egy is extended to Multiple-Input-Multiple-Output (MIMO), (ii) the influence of the sensor noise levels is included in the analysis and (iii) the systems performance is validated for 3D disturbances.

This work presents the validation of a measurement device with integrated active vibration isolation. The device serves as a proof of principle for multiple vibration isolation strategies, which will be discussed in this work. In section 4.2 a simple 1D model of a CMFM with integrated AVIC is analysed. Section 4.3 , presents two vibration isolation techniques: feedback and feedforward. The optimal parameters to achieve the maximum vibration isolation performance are investigated for both strategies. In section 4.4 the design of the proof-of-principle mechanism is discussed. The CMFM with integrated AVIC is validated for both strategies in section 4.5. A discussion is provided in section 4.6 and finally the main conclusions are summarised in section 4.7

\subsection{D modelling}

In this section a simple, but accurate model of a CMFM with integrated active vibration isolation is analysed. The simple model is used to derive and illustrate the vibration isolation strategies. In Chapter 2 a complex model of a CMFM is reduced to a 1D mass-spring system for the relevant Coriolis force induced motion, the swing motion, as shown in Fig. 4.1. This model is depicted in Fig. 4.2 and describes the influence of external vibrations $a_{1}$ and the Coriolis effect $F_{\text {cor }}$ (Eq. 4.1) on the displacement of the tube $y_{\text {cor }}=y_{2}-y_{1}$, from which the mass-flow measurement is derived. The tube properties of the experimental setup 1 are $m_{2}=2.45 \times 10^{-5} \mathrm{~kg}, d_{2}=1.60 \times 10^{-5} \mathrm{~N} \mathrm{~s} \mathrm{~m}^{-1}$ and $k_{2}=10.4 \mathrm{~N} \mathrm{~m}^{-1}$, resulting in a relatively undamped $\left(\mathrm{Q}\right.$-factor $\approx 1000$ ) resonance frequency $\omega_{2}=103.7 \cdot 2 \pi \mathrm{rad} / \mathrm{s}$. The actuation mode, an oscillation around the $\theta_{\text {twist }}$-axis with a resonance frequency $\omega_{\text {act }}=175 \cdot 2 \pi \mathrm{rad} / \mathrm{s}$, is not included in this simplified model. Only the Coriolis force $F_{\text {cor }}$, which is at the actuation frequency, is included in the model.

\footnotetext{
${ }^{1}$ Note that the tube geometry in this chapter is different than in Chapter 2 and therefore the presented resonance frequencies are higher.
}

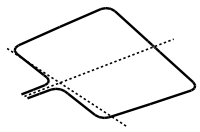




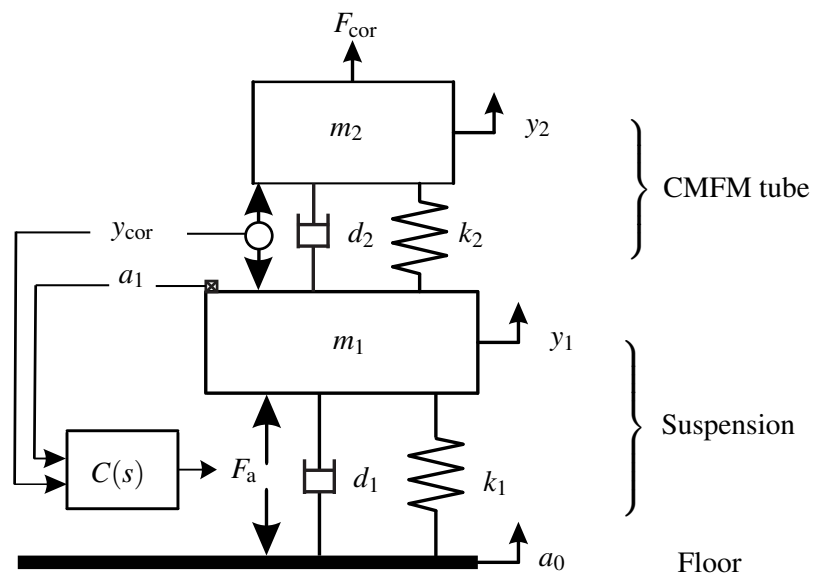

Fig. 4.3 Mass-damper-spring model: 1D representation of multi-DOF suspension system. For passive vibration isolation the force $F_{\mathrm{a}}$ and controller $C(s)$ are omitted. Feedback uses the sensor signal $a_{1}$ and the feedforward strategy uses also the sensor signal $a_{0}$.

The displacement $y_{\text {cor }}$, expressed in the Laplace domain, is equal to:

$$
y_{\text {cor }}(s)=\frac{-1}{s^{2}+\frac{d_{2}}{m_{2}} s+\frac{k_{2}}{m_{2}}} a_{1}(s)+\frac{1}{m_{2} s^{2}+d_{2} s+k_{2}} F_{\text {cor }}(s),
$$

this displacement is dependent on the external vibrations $a_{1}$ and the Coriolis force $F_{\text {cor }}$, which is proportional to the mass-flow $\dot{\Phi}_{\mathrm{m}}(\mathrm{Eq} .4 .1)$. The mass-flow measurement value is calculated from the phase-difference between two tube displacement sensors on equal distance of the $\theta_{\text {twist }}$-axis. The phase-difference is determined by a phase demodulation algorithm, using only information around the actuation frequency (Chapter 2).

An external vibration with a frequency content around the actuation frequency has a direct influence on the mass-flow measurement value (Chapter 2). The transmissibility from $a_{1}$ to $y_{\text {cor }}$ describes this influence. Minimising this transmissibility, without affecting the transfer function of $F_{\text {cor }}$ to $y_{\text {cor }}$, results in a reduction in the sensitivity for external vibrations. The attenuation is only needed in a relatively small $(50 \mathrm{~Hz})$ frequency band around the actuation frequency, because the influence outside this band can easily filtered out of the sensor signals, when calculating the mass-flow. Therefore, only attenuation around the actuation frequency $\omega_{\text {act }}$ is needed. This is indicated by a $50 \mathrm{~Hz}$ wide region of interest (ROI) in all the bode diagrams in this paper.

A possible solution is to add a flexible suspension between de CMFM tube and the floor. This results in an extension of Fig. 4.2 as depicted in Fig. 4.3. The acceleration of the newly introduced stage can be described as:

$$
a_{1}(s)=\frac{d_{1} s+k_{1}}{m_{1} s^{2}+d_{1} s+k_{1}} a_{0}(s)+\frac{s^{2}}{m_{1} s^{2}+d_{1} s+k_{1}} F_{\mathrm{a}}(s),
$$

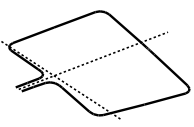


where the dynamics of the tube are neglected, which is only valid if $m_{2} \ll m_{1}$. When also neglecting the actuator $F_{\mathrm{a}}$, we have a form of passive vibration isolation [53]. However, the performance is insufficient, because the suspension frequency has a lower bound, limiting the attenuation. The frequency is limited by the maximum stress in the connection tubes and a maximum allowable sag of the stage due to gravity. Therefore sensors and actuators are added to the model for active vibration isolation control (AVIC). In our model, we choose for a suspension mode with a resonance frequency of $\omega_{1}=30 \cdot 2 \pi \mathrm{rad} / \mathrm{s}$. Given the stage mass $m_{1}=0.2 \mathrm{~kg}$ and relative damping $\zeta=0.01$, this results in $d_{1}=0.75 \mathrm{~N} \mathrm{~s} \mathrm{~m}^{-1}$ and $k_{1}=$ $7.1 \times 10^{3} \mathrm{~N} \mathrm{~m}^{-1}$.

The actuator is operated in voltage-mode in order to obtain the least amount of actuator noise, resulting in an additional pole in the transfer function, which is dependent on the motorconstant $k_{\mathrm{m}}$, induction $L$ and resistance $R$ of the actuator coil:

$$
F_{\mathrm{a}}(s)=\frac{k_{\mathrm{m}}}{L s+R} U(s) \approx \frac{\omega_{\text {ind }}}{s+\omega_{\text {ind }}} U(s),
$$

whereby the low frequency gain is assumed to be 1 . The model for control can be summarised as follows. A primary path, also called the transmissibility:

$$
P(s)=\frac{a_{1}(s)}{a_{0}(s)}=\frac{d_{1} s+k_{1}}{m_{1} s^{2}+d_{1} s+k_{1}},
$$

and a secondary path, the actively controlled part:

$$
S(s)=\frac{a_{1}(s)}{U(s)}=\frac{s^{2}}{m_{1} s^{2}+d_{1} s+k_{1}} \cdot \frac{\omega_{\text {ind }}}{s+\omega_{\text {ind }}},
$$

both result in an acceleration $a_{1}$, which needs to be minimised to reduce the influence of external vibrations on the mass-flow measurement. Both transfer functions are depicted in Fig. 4.5

\subsection{Control design}

Actively reducing the influence of external vibrations can be done in several ways. In this section a feedback and an adaptive feedforward strategy are compared on the ability to reduce the transmissibility and to handle sensor noise. For clarity, the model and the control strategies are presented SISO. Only the adaptive algorithm is presented in MIMO, since this is not straightforward.

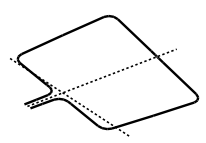




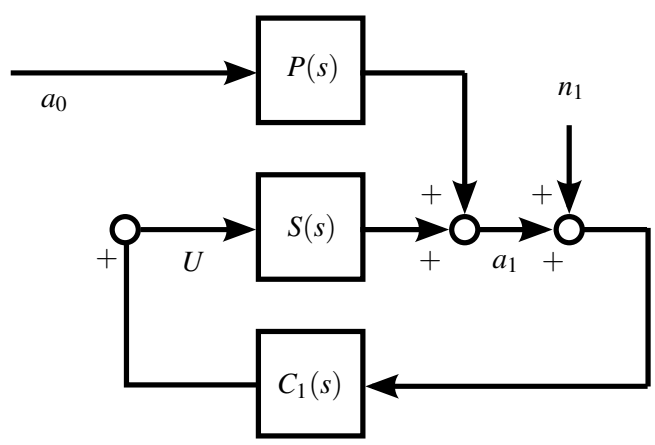

Fig. 4.4 Feedback scheme, based on Eq.4.3 and 4.7 Noise $n_{1}$ is added to the acceleration sensor, measuring $a_{1}$.

\subsubsection{Feedback}

Reconsider the model in Fig. 4.3. The transmissibility from external vibrations $a_{0}$ to the Coriolis displacement $y_{\text {cor }}$ gives the influence of external vibrations on the measurement value of a CMFM (Chapter 2). In Chapter 3] and in more detail in [65], a strategy is presented to use acceleration feedback to add virtual mass and skyhook damping to $m_{1}$. This results in a lower suspension frequency and thus a lower transmissibility. The expression for the controller is given by:

$$
C_{1}(s)=\frac{U(s)}{a_{1}(s)}=-\left(K_{\mathrm{a}}+\frac{K_{\mathrm{v}}}{s}\right) \underbrace{\frac{s^{2}}{s^{2}+2 \zeta_{\mathrm{f}} \omega_{\mathrm{f}} s+\omega_{\mathrm{f}}^{2}}}_{H_{1}(s)} \underbrace{\frac{\omega_{\mathrm{r}}^{2}}{s^{2}+2 \zeta_{\mathrm{r}} \omega_{\mathrm{r}} s+\omega_{\mathrm{r}}^{2}}}_{H_{2}(s)} \underbrace{\frac{s+\omega_{\mathrm{z}}}{\omega_{\mathrm{Z}}}}_{H_{3}(s)},
$$

where $K_{\mathrm{a}}=0.172$ is the added virtual mass and $K_{\mathrm{v}}=30.8$ the added skyhook damping to damp the suspension mode with frequency $\omega_{1}$. The gains $K_{\mathrm{a}}$ and $K_{\mathrm{v}}$ are calculated as followed [77]:

$$
K_{\mathrm{a}}=m_{1}\left(\frac{\omega_{1}^{2}}{\omega_{\text {new }}^{2}}-1\right), \quad K_{\mathrm{v}}=2 \zeta_{\text {new }} m_{1} \frac{\omega_{1}^{2}}{\omega_{\text {new }}},
$$

where $\omega_{\text {new }}=22 \cdot 2 \pi \mathrm{rad} / \mathrm{s}$ is the new frequency of the suspension with a relative damping $\zeta_{\text {new }}=0.3$. The term $H_{1}(s)$ is a second-order high-pass filter with a corner frequency $\omega_{\mathrm{f}}=$ $1 \cdot 2 \pi \mathrm{rad} / \mathrm{s}$ and $\zeta_{\mathrm{f}}=0.7$, used to prevent actuator saturation. The term $H_{2}(s)$ is an slightly damped second-order low-pass filter at the tube actuation frequency $\omega_{\mathrm{r}}=175 \cdot 2 \pi \mathrm{rad} / \mathrm{s}$. This filter limits the control bandwidth and adds extra attenuation in the region of interest due to a low $\zeta_{\mathrm{r}}=0.07$. The term $H_{3}(\mathrm{~s})$ is a zero at $\omega_{\mathrm{z}}=200 \cdot 2 \pi \mathrm{rad} / \mathrm{s}$ and is added to increase the phase-margin at the cross-over frequency, around $300 \mathrm{~Hz}$.

The strategy is depicted schematically in Fig. 4.4, the plant and controller are depicted in Fig. 4.5 and 4.6 respectively. The resulting transmissibility is depicted in Fig. 4.9, show-

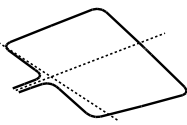



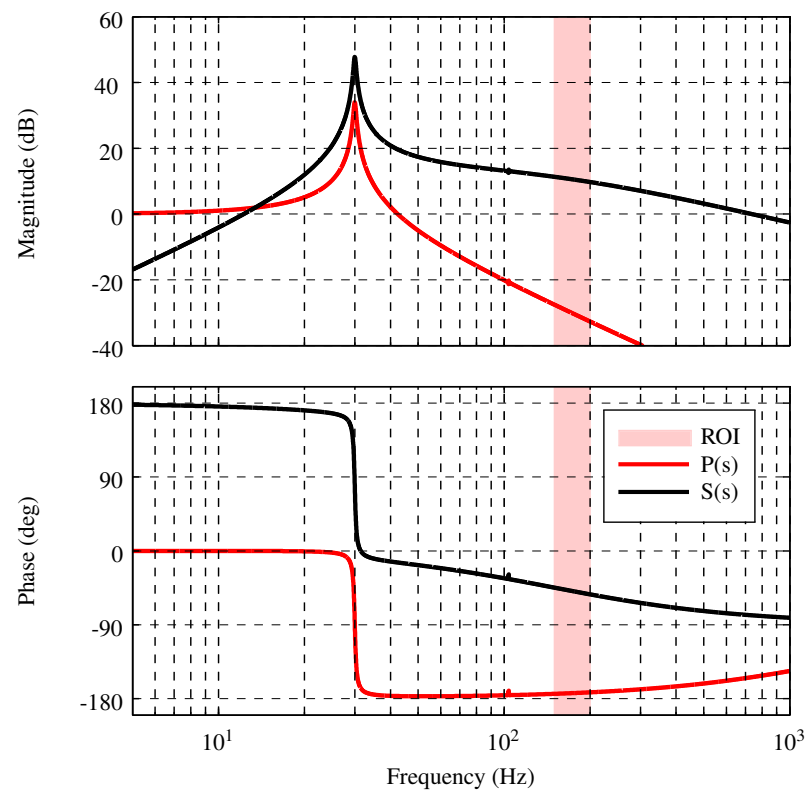

Fig. 4.5 Model of the plant design, the primary and secondary path according to Eq. 4.5 and 4.6

ing an attenuation of $50 \mathrm{~dB}$ in the region of interest. The strategy has disadvantages: High performance requires a high controller bandwidth, but the bandwidth is limited by the high frequency dynamics of the system, which might induce instability. Therefore, knowledge of the high-frequency system dynamics is required to guarantee a robust and stable system. In the validation of the mechanical design, the MIMO system is uncoupled to multiple SISO systems, all using the SISO strategy presented in this subsection.

\subsubsection{Adaptive feedforward}

Alternatively a feedforward strategy can be applied. An extra sensor measures the external vibrations $a_{0}$, see Fig. 4.3, and this signal is used for compensation of the stage movements. The schematic is depicted in Fig. 4.7. Optimal compensation is achieved with the following controller:

$$
C_{\mathrm{FF}}(s)=\frac{U(s)}{a_{0}(s)}=-S^{-1}(s) P(s)=-\frac{d_{1} s+k_{1}}{s^{2}} \cdot \frac{s+\omega_{\text {ind }}}{\omega_{\text {ind }}}
$$

Since $P(s)$ and $S(s)$ have poles in common, they cancel out. Therefore, the controller is only dependent on the physical parameters - stiffness, damping and actuator dynamics - between the floor and the suspended stage, no knowledge of the internal dynamics is needed. This can 

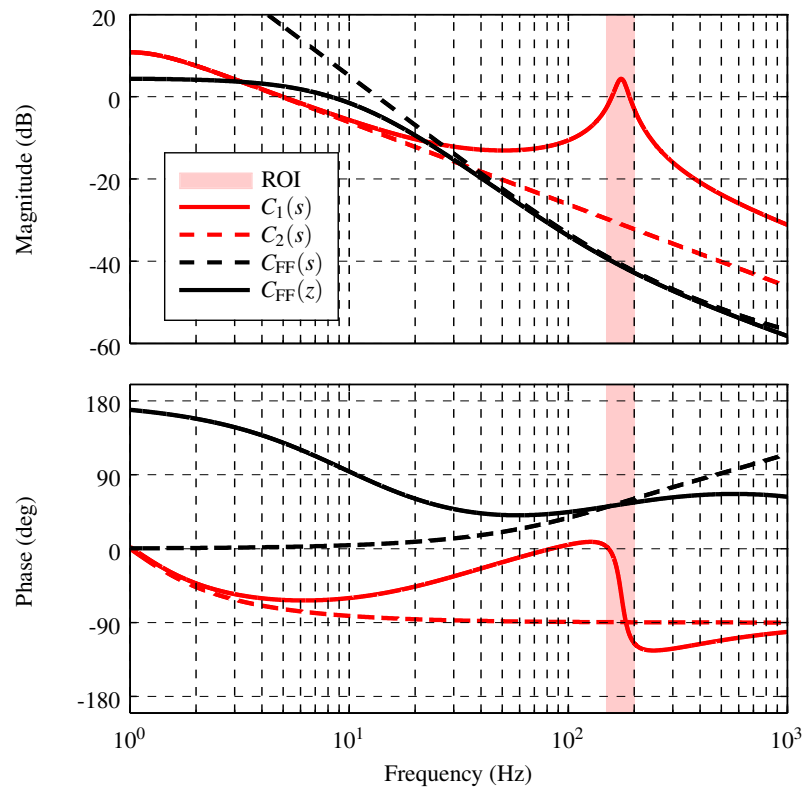

Fig. 4.6 Controller design of both the feedback and feedforward strategy (Eq.4.7,4.9 and 4.18).

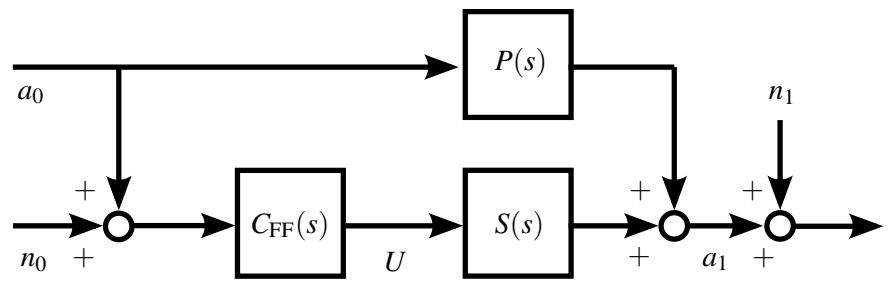

Fig. 4.7 Feedforward scheme. Noise is added to the acceleration sensors.

be understood conceptually as follows; by compensating the forces due to the stiffness and damping, no forces are transmitted from the floor to $m_{1}$. Note that the zeros of $P(s)$ and $S(s)$ are not necessarily the same and can appear as pole-zero in the controller structure. This is of importance when attenuation in a large frequency band is needed. The controller (Eq. 4.9) can be written as a series of Infinite Impulse Response (IIR) filters with ideal parameters:

$$
C_{\mathrm{FF}}(s)=\boldsymbol{w} \boldsymbol{F}(s)=\left[-k_{1}-\left(d_{1}+\frac{k_{1}}{\omega_{\text {ind }}}\right)-\frac{d_{1}}{\omega_{\text {ind }}}\right]\left[\begin{array}{c}
\frac{1}{s^{2}} \\
\frac{1}{s} \\
1
\end{array}\right]
$$

In practice, only estimated parameters of the weights $\boldsymbol{w}$ are available. In this paper we propose a filtered-reference least-mean-square (FxLMS) algorithm with residual noise shap-

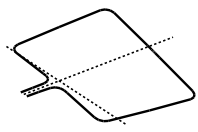




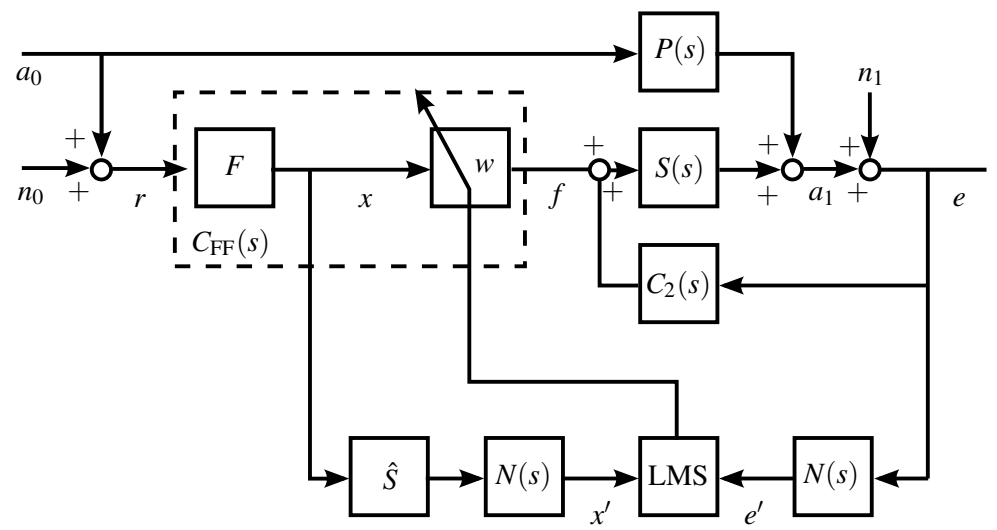

Fig. 4.8 Modified FxLMS adaptive feedforward control scheme, an extension of the schema in Fig.4.7

ing [4, 36] to update the weights. The weights are adapted such that the squared error is minimal, resulting in an optimal feedforward controller. The algorithm is explained in the remaining part of this subsection. The scheme is depicted in Fig.4.8 The algorithm minimises the following quadratic cost function:

$$
J(n)=e^{\prime}(n)^{T} e^{\prime}(n),
$$

with $n$ the iteration step. The filtered error is given by:

$$
e^{\prime}(n)=N e(n)=N(P+S w \boldsymbol{F}) a_{0}(n),
$$

whereby the controller $C_{2}(s)$ is omitted. This controller will be introduced at the end of this subsection. The error is filtered using the filter $N(s)$ in order to minimise the error in only a small frequency band. $N(s)$ is defined as a $50 \mathrm{~Hz}$ bandpass filter between 150 and $200 \mathrm{~Hz}$ (the region of interest). The weights are determined, using the following gradient-based update law [36]:

$$
\boldsymbol{w}(n+1)=\boldsymbol{w}(n)-\frac{\mu(n)}{2}\left(\frac{\partial J(n)}{\partial \boldsymbol{w}(n)}\right)^{T}
$$

For updating the weights, the gradient of the quadratic cost function is needed:

$$
\frac{\partial J(n)}{\partial \boldsymbol{w}(n)}=\frac{\partial J(n)}{\partial e^{\prime}(n)} \frac{\partial e^{\prime}(n)}{\partial \boldsymbol{w}(n)} \approx 2 e^{\prime}(n) N S \boldsymbol{x}(n)=2 e^{\prime}(n) \boldsymbol{x}^{\prime}(n),
$$

where $\boldsymbol{x}=\boldsymbol{F} a_{0}$ is the filtered reference signal $a_{0}$. Merging (4.13) and (4.14) gives the update law:

$$
\boldsymbol{w}(n+1)=\boldsymbol{w}(n)-\mu(n) \boldsymbol{x}^{\prime}(n)^{T} e^{\prime}(n),
$$

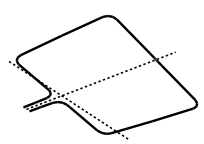


with adaptation rate $\mu(n)$, depending on the reference signals, [36]:

$$
\mu(n)=\frac{\bar{\mu}}{\varepsilon+\boldsymbol{x}^{\prime T}(n) \boldsymbol{x}^{\prime}(n)},
$$

where $\varepsilon>0$ is a small value to prevent division by zero and $\bar{\mu}$ the adaptation rate to prevent instability of the adaptation process. To determine $\boldsymbol{x}^{\prime}(n)$ the secondary path $S(s)$ is needed. The multiplication with the secondary path is needed to align the error and reference signals in time. The estimation of the secondary path is not required to be very accurate, the stability of adaptive algorithms is assured for a phase error even up to $\pm 90^{\circ}[17,49]$. Therfore, a non-dynamic gain matrix with real numbers is used as estimate $\hat{S}$, because the phase of $S(s)$ is approximated to be constant in the small frequency band of $N(s)$. Note that this approximation results in a lower convergence of the weights. A discrete-time formulation of the IIR filter (Eq.4.10) is proposed, with tame integrators to prevent drift and actuator saturation, and the IIR filters have fixed poles to make the adaptation inherently stable, as:

$$
\boldsymbol{F}(z)=\left[\frac{\omega_{\mathrm{x}}^{2} T_{\mathrm{s}}^{2}}{\left(z-\left(1-\alpha T_{\mathrm{s}}\right)\right)^{2}} \frac{\omega_{\mathrm{x}} T_{\mathrm{s}}}{\left(z-\left(1-\alpha T_{\mathrm{s}}\right)\right)} 1\right],
$$

where $T_{\mathrm{s}}$ is the sample time and the integrators have a cut-off frequency at $\alpha=10 \cdot 2 \pi \mathrm{rad} / \mathrm{s}$. The gain $\omega_{\mathrm{x}}$ is the center frequency of the bandpass filter $N(s)$, this scaling factor is chosen such that the power of each of the signals in the vector $\boldsymbol{x}^{\prime}(n)$ is equal. The residual noise filter $N(s)$ tunes the weights such that the transmissibility is minimal in the region of interest. The ideal controller $C_{\mathrm{FF}}(s)$ (Eq. 4.9) is compared, in Fig. 4.6, to the controller $C_{\mathrm{FF}}(z)=\boldsymbol{w} \boldsymbol{F}(z)$ , whereby the weights are obtained using the update law above (Eq. 4.15). In the region of interest the phase and gain of the controllers are equal.

Further, damping of the suspension mode is desirable, therefore a simple feedback skyhook damper is added to the feedforward strategy:

$$
C_{2}(s)=\frac{U(s)}{a_{1}(s)}=-\frac{K_{\mathrm{v}}}{s} \cdot H_{1}(s)
$$

where $K_{\mathrm{v}}=30.8$ is the added sky-hook damping and the second-order high-pass filter $H_{1}(s)$ limits the controller to prevent integrator saturation. The controller $C_{2}(s)$ is depicted in Fig. 4.6 Compared to the controller $C_{1}(s)$ the controller has only a high gain for low frequencies. The result of the feedforward strategy is discussed in section 4.3.3. 


\subsubsection{MIMO formulation}

The feedforward strategy is presented in SISO, but for the implementation a MIMO formulation is needed, because there are multiple reference and error sensors and multiple actuators in the experimental setup. Therefore the following formulation, introduced by [7], is used:

$$
\begin{aligned}
x_{i, l}(n) & =F_{l} r_{i}(n) \\
x_{i, j, k, l}^{\prime}(n) & =N \hat{S}_{j, k} x_{i, l}(n) \\
\mu(n) & =\frac{\bar{\mu}}{\varepsilon+x_{i, j, k, l}^{\prime}(n) x_{i, j, k, l}^{\prime}(n)} \\
w_{i, j, l}(n+1) & =w_{i, j, l}(n)-\mu(n) x_{i, j, k, l}^{\prime}(n) e_{k}(n) \\
f_{j}(n) & =w_{i, j, l}(n) x_{i, l}(n)
\end{aligned}
$$

where:

$I$ : number of reference signals

$J$ : number of actuators

$K:$ number of error sensors

$L$ : number of weights in each filter

$r_{i}:$ reference signal

$e_{k}:$ error signal

$\hat{S}_{j, k}$ : estimate of the secondary path

$w_{i, j, l}$ : weights

$f_{j}(n)$ : feedforward actuator input

\subsubsection{Model results}

In the previous subsections two active strategies are presented to reduce the influence of external vibrations on the mass-flow measurement value of a CMFM. In Fig. 4.9 the transmissibility from external vibrations $a_{0}$ to the compliantly suspended stage accelerations $a_{1}$ is depicted for all strategies. The best attenuation in the region of interest is achieved using the feedforward strategy, which gives an attenuation of more than $50 \mathrm{~dB}$.

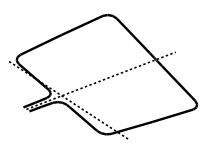




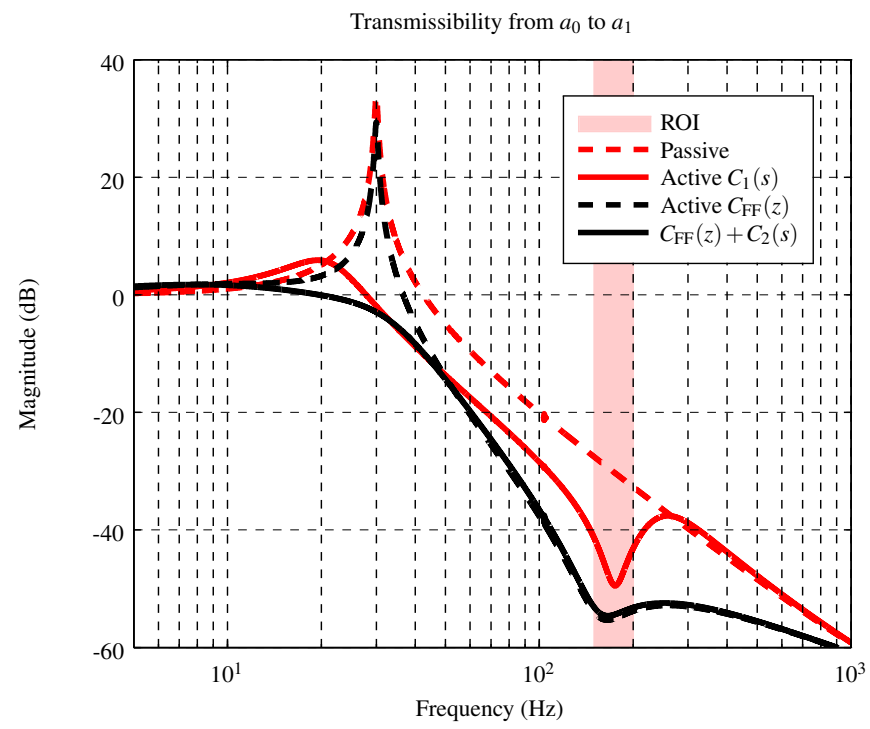

Fig. 4.9 Modelled transmissibilities from $a_{0}$ to $a_{1}$ for the passive and active systems

\subsubsection{Limitations by sensor noise}

AVIC is able to reduce the influence of external vibrations. However, the noise of the additional acceleration sensors may be sources of extra disturbances. The sensitivity for sensor noise is determined in this section.

The effect of the noise of the sensors measuring the external and stage vibrations, respectability $a_{0}$ and $a_{1}$, is considered. In the analysis, the noise signals $n_{0}$ and $n_{1}$ are added in the feedback and feedforward strategies in Fig. 4.4 and 4.8. For the feedback strategy, the sensitivity is equal to:

$$
S_{\mathrm{FB}}=\frac{a_{1}(s)}{n_{1}(s)}=\frac{S(s) C_{1}(s)}{1-S(s) C_{1}(s)}
$$

In the fixed-gain feedforward strategy, there are two sensitivity functions, because there are two types of sensors:

$$
\begin{aligned}
& S_{\mathrm{FF}, 0}=\frac{a_{1}(s)}{n_{0}(s)}=\frac{S(s) C_{\mathrm{FF}}(s)}{1-S(s) C_{2}(s)} \\
& S_{\mathrm{FF}, 1}=\frac{a_{1}(s)}{n_{1}(s)}=\frac{S(s) C_{2}(s)}{1-S(s) C_{2}(s)}
\end{aligned}
$$

The sensitivity functions are depending on the secondary path (Eq. 4.6) and the designed controllers (Eq. 4.7/4.10 and 4.18). All three sensitivity functions are depicted in Fig. 4.10.

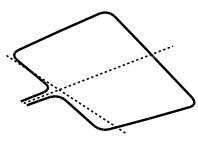


For the feedback strategy the sensitivity is close to unity in the region of interest, because the feedback tries to compensate for the sensor noise, imposing the stage to move in antiphase with the noise. The sensitivity for the fixed-gain feedforward strategy is much lower, because it compensates for $a_{0}$ and its effect is thus reduced by the attenuation of the passive stage. Thus, the requirements on the noise-level for the fixed-gain feedforward strategy can be significantly higher than for the feedback strategy. For the adaptive feedforward strategy the weights are obtained by minimising a cost function (Eq. 4.11), therefore the quality of the weights is dependent on the noise of the error sensor. An error in the weights introduces an error in the compensation and thus the attenuation of the adaptive feedforward scheme is dependent on the ratio between error sensor noise and the level of external vibrations.

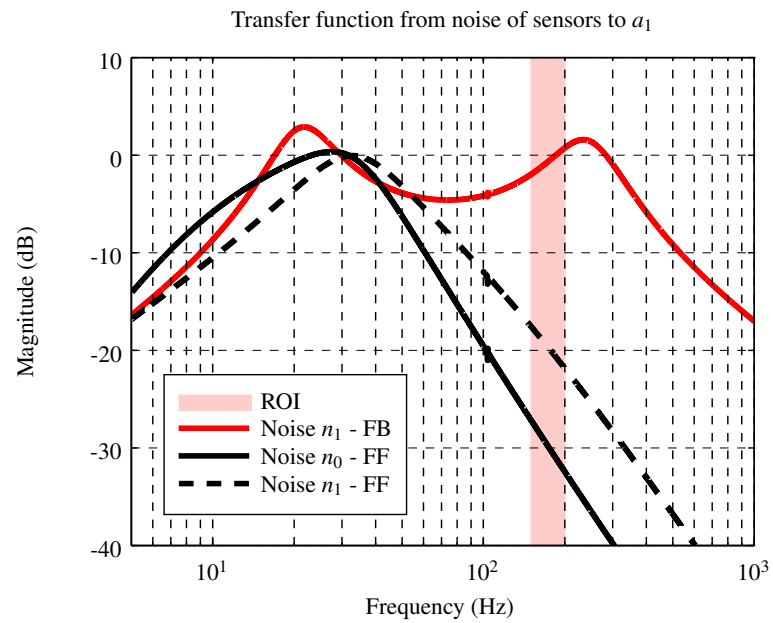

Fig. 4.10 Sensitivity transfer functions from sensor noise to stage accelerations $a_{1}$ (Eq.4.19,4.20 and 4.21).

The previous analysis can be used to select proper acceleration sensors for the active vibration isolation strategies. The contribution of the acceleration sensor noise to the Coriolisdisplacement should be less than the noise already present in the measurement of the Coriolisdisplacement, which is $3.16 \mathrm{e}-10 \mathrm{~m} / \sqrt{\mathrm{Hz}}$. The influence of external vibrations and the noise levels of the acceleration sensors on the tube displacement sensor $y_{\text {cor }}$ is obtained from the following relation:

$$
\Phi_{y_{\mathrm{cor}}}=\left|T_{y_{\mathrm{cor}}, a_{1}}(s) S_{a_{1}, n}(s)\right|^{2} \cdot \Phi_{n}+\left|T_{y_{\mathrm{cor},}, a_{1}}(s) P_{a_{1}, a_{0}}(s)\right|^{2} \cdot \Phi_{a_{0}},
$$

where $\Phi$ is the Power Spectral Density of respectively the contribution to the error in $y_{\text {cor }}$, the acceleration sensor noise $n$ and the external vibrations $a_{0}, S_{a_{1}, n}$ is one of the above sensitivity functions (Eq. 4.19, 4.20 and 4.21) and the transmissibility $T_{y_{\mathrm{cor}}, a_{1}}$ is given in Eq. 4.2. The result of Eq. 4.22 at the actuation frequency of $175 \mathrm{~Hz}$, is depicted in Fig. 4.11 From the first figure it can be concluded that for the feedback strategy a sensor with a noise level of less than $25 \mu \mathrm{g} / \sqrt{\mathrm{Hz}}$ is needed, without affecting the Coriolis displacement. For the feedforward

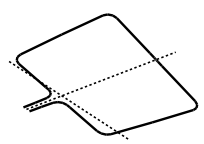




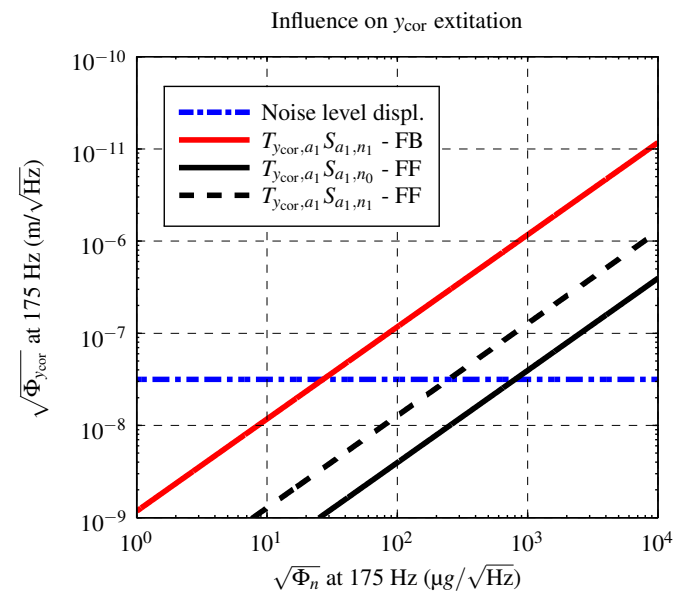

(a) Sensors noise level influence

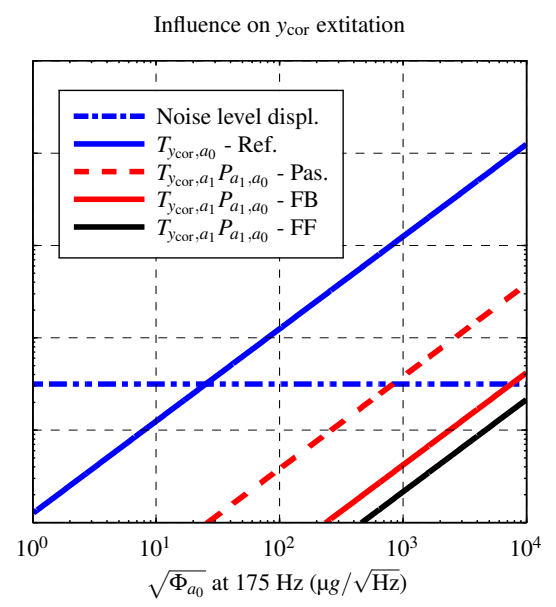

(b) External vibrations influence

Fig. 4.11 Influence of external vibrations (b) and the acceleration sensors noise levels (a) on the PSD of $y_{\text {cor }}$ (Eq.4.22), compared to the noise level of the displacement sensor. The noise level of the displacement sensors is $1 \mathrm{e}-13 \mathrm{~mm}^{2} / \mathrm{Hz}=3.16 \mathrm{e}-10 \mathrm{~m} / \sqrt{\mathrm{Hz}}$ at $175 \mathrm{~Hz}$.

strategy the noise level can be higher, respectively $800 \mu \mathrm{g} / \sqrt{\mathrm{Hz}}$ and $250 \mu \mathrm{g} / \sqrt{\mathrm{Hz}}$ for the measurement of $a_{0}$ and $a_{1}$. In the second figure is shown, that the passive and active strategies have an tremendous effect on reducing the influence of external vibrations.

\subsection{Mechanism design}

An experimental setup is needed to validate the control designs presented in Section 4.3 In Section 4.2 a simple mass-spring 1D model of a CMFM including AVC was presented. In the first subsection the multi-DOF concept is explained and dimensions are presented in the second subsection. Thirdly, the chosen actuators and sensors are presented. In the final subsection the multi-DOF model results are compared to the 1D model. Details of the modelling and design of the mechanism are presented in more detail in Staman [58].

\subsubsection{Conceptual design}

Based on the 1D model, shown in Fig. 4.3, a multi-DOF concept is developed. Because the vibration sensitivity of the CMFM is dominated by two directions, a $y$-translation and a $x$-axis rotation (see Fig. 4.1), both directions will be passively suspended. Furthermore, the setup will be used to test if the tube-window can be actuated by the stage. In total, this results in a

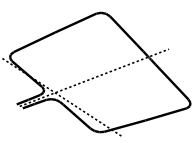




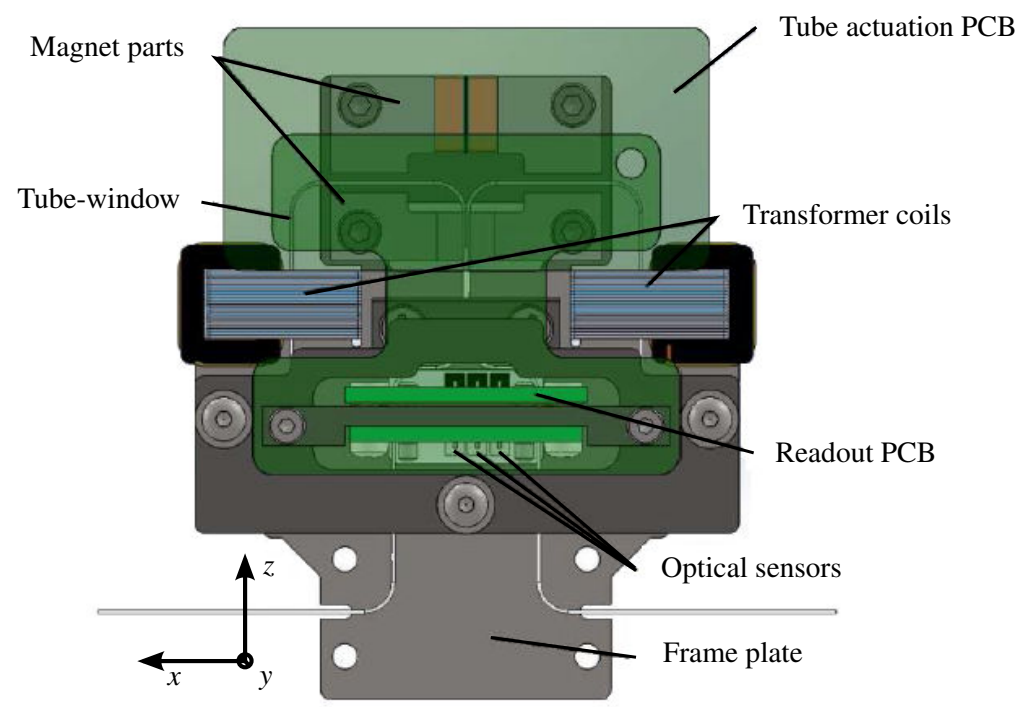

Fig. 4.12 SolidWorks model of the measurement stage [59] (Note that the top PCBs are made transparent for clarity). The functional part of a CMFM, used as a module in the CMFM with integrated active vibration isolation.

3-DOF stage, whereby the out-of-plane directions are passively suspended. Active means are added to make the 3-DOF motions measurable and controllable The attenuation is a function of the suspension frequency (Chapter 3). The designed suspension frequency will be $30 \mathrm{~Hz}$, to limit the maximum stresses in the connection tubes and a maximum allowable sag due to gravity.

\subsubsection{Dimensional design}

The design of a Coriolis Mass-Flow Meter with integrated Active Vibration Isolation Control is based on a patented design ( [45, 46]) of an existing CMFM for low flows. Therefore, a functional model of a CMFM is striped from the casing to only the core of the instrument; the measurement stage. In Fig. 4.12 this stage is shown. It contains a frame plate with mounted on it: a tube-window (Fig. 4.1), actuation means to actuate the tube-window and optical displacement sensors to measure the tube displacements. The measurement stage has a mass of about $110 \mathrm{~g}$.

The measurement stage is compliantly suspended in 3 directions, an exact constraint design [57]. The 3-DOF type 1 suspension [30] is depicted in Fig. 4.13(a). The length, height and width of each flexure are chosen such that the stage is suspended around $30 \mathrm{~Hz}$ in all three out of the $x z$-plane directions.

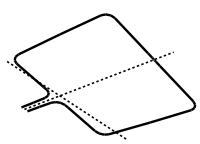



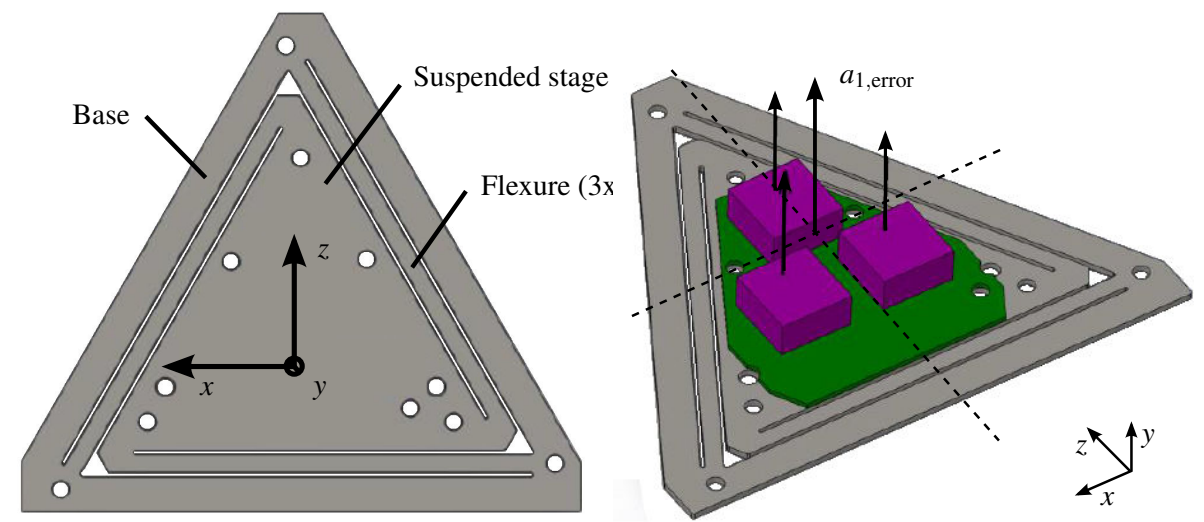

(a) Solidworks model of the 3 DOF suspension.

(b) Solidworks model of the acceleration sensors (purple) and PCB (green) on the suspension. Including the location of the error sensor $a_{1, \text { error }}$.
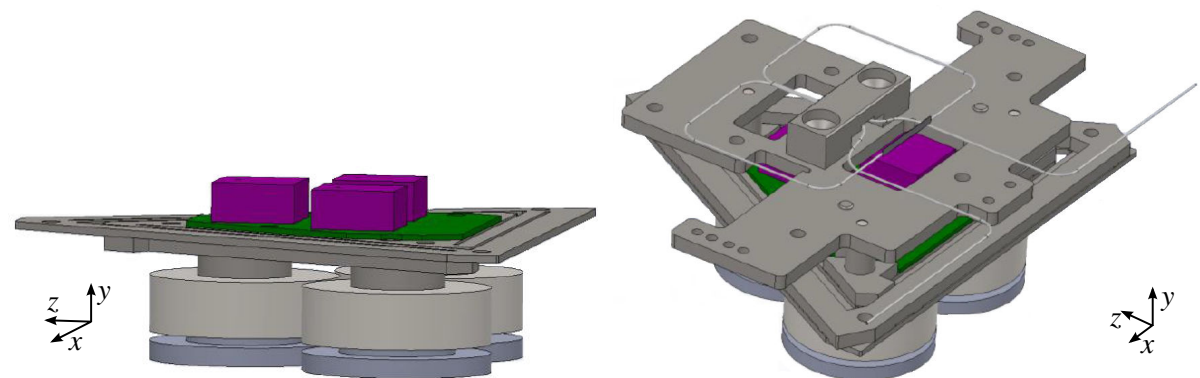

(c) Solidworks model of the actuator locations.

(d) Solidworks model of the active vibration isolation system with a simplified measurement stage (Fig.4.12)

Fig. 4.13 Design of CMFM with integrated active vibration isolation [59].

On the suspension a PCB with three accelerometers is mounted to measure the stage accelerations $\boldsymbol{a}_{1}$ (Fig. 4.13(b) Below the suspension three voice coil actuators are mounted to control the stage movements (Fig. 4.13(c) ). The measurement stage (Fig. 4.12) is mounted on top of the suspension as shown in Fig. 4.13(d) A photo of the final setup is shown in Fig.4.16 


\subsubsection{Sensor and actuator choice}

In Fig. 4.13(c), the suspension with the voice coil actuators and acceleration sensors is shown. For the stage, small and low noise acceleration sensors are required. The effect of sensor noise is discussed in subsection 4.3.3.1. The used one-axis acceleration sensors are Silicon Design $1221-2 g$ sensors, selected on their size $(9 \times 9 \times 3 \mathrm{~mm})$, their noise performance $(5 \mu \mathrm{g} / \sqrt{\mathrm{Hz}})$ and their relatively low costs.

The voice coil motors are Lorentz type actuators and are designed in-house. One actuator is able to produce a force of about $0.8 \mathrm{~N}$ at a current of $1 \mathrm{~A}$, while the resistance of the coil is approximately $4.2 \Omega$ and the coil inductance is about $L=4.5 \mathrm{mH}$. Further, it has a diameter of $20 \mathrm{~mm}$ and a height of about $10 \mathrm{~mm}$.

\subsubsection{Model results}

The entire system has been modelled using the non-linear finite element flexible multibody software package SPACAR [31]. The real experimental parameters are implemented in the model. Modal analysis is used for tuning the suspension design. The multibody package produces the input-output relations, those transfer functions are used for the necessary control synthesis [32]. First, the transfer functions of external vibrations to the stage movement are determined and depicted in Fig. 4.14 Whereby the applied external vibriations $\boldsymbol{a}_{0}$ consist of three translations and three rotations. As output an acceleration of the platform in $y$-direction is chosen (see Fig. 4.13(b), which is defined as:

$$
a_{1, \mathrm{error}}=[-0.2516,0.6258,0.6258] \boldsymbol{a}_{1}=\boldsymbol{R}_{\mathrm{y}} \boldsymbol{a}_{1},
$$

where $\boldsymbol{a}_{1}$ is a vector with the top, left and right sensor of the stage (Fig 4.13(b)). This point is chosen because it is the percussion point of the tube-window. Only a $y$-translation in this point will result in a Coriolis displacement. Fig. 4.14 shows clearly that the resonance frequencies of the suspension are around $30 \mathrm{~Hz}$. For the $y$-disturbance, which is the dominant direction at low frequencies, the attenuation in the region of interest (around the tube actuation frequency of $175 \mathrm{~Hz}$ ) is as expected. However, this direction is not the dominant direction in the region of interest any more. A disturbance in $z$-direction is causing the stage to tilt and results in an acceleration in the $y$-direction at the sensor positions. This occurs due to the fact that the centre of mass and the centre of compliance are not in the same position for the realised mechanism.

For the application the direction of the disturbance is unknown and can alternate. Because the direction is frequency dependent, a Singular Value Decomposition (SVD) of the transmissibility function is performed for each frequency. The maximum singular value as function of the frequency is shown in Fig. 4.15. The maximum singular value for the transmissibility is also shown for each of the vibration isolation strategies of section 4.3, which have been extended to MIMO. The gained attenuation for the active strategies is with respect to the passive system as expected from the 1D model (Fig. 4.9). But the total attenuation is less than 


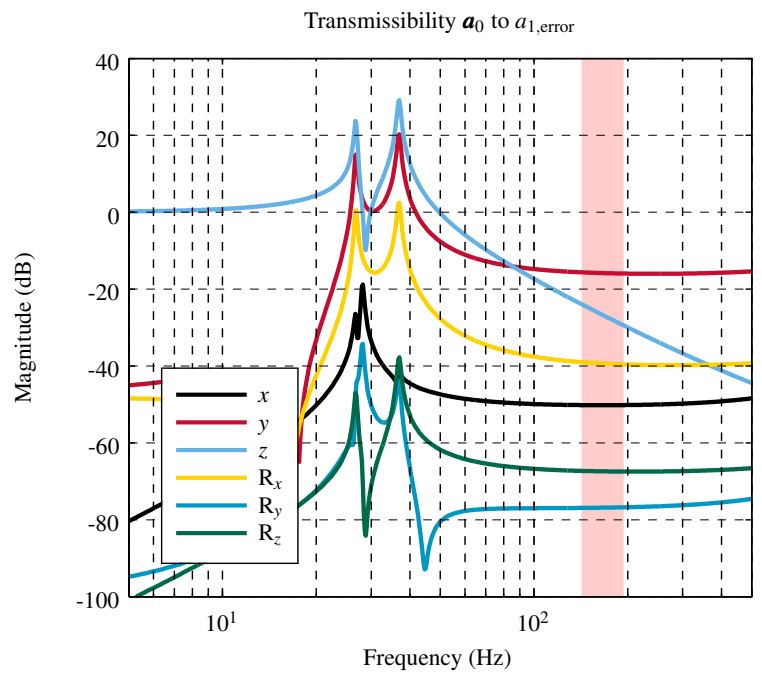

Fig. 4.14 Modelled passive transmissibility from $\boldsymbol{a}_{0}$ to $a_{1 \text {,error }}$ of the 3-DOF stage.

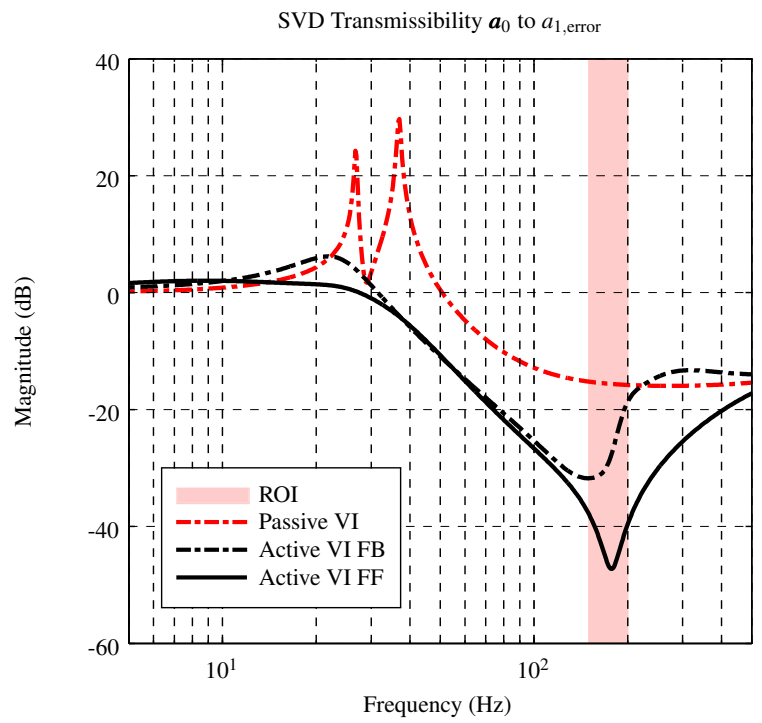

Fig. 4.15 Modelled active SVD transmissibility from $\boldsymbol{a}_{0}$ to $a_{1 \text {,error }}$ for the passive and active vibration isolation (VI). Whereby the attenuation in the region of interest is minimised.

the expected $50 \mathrm{~dB}$, because the flexible suspension of the stage has a dominant direction that has not been taken into account in the 1D model. 


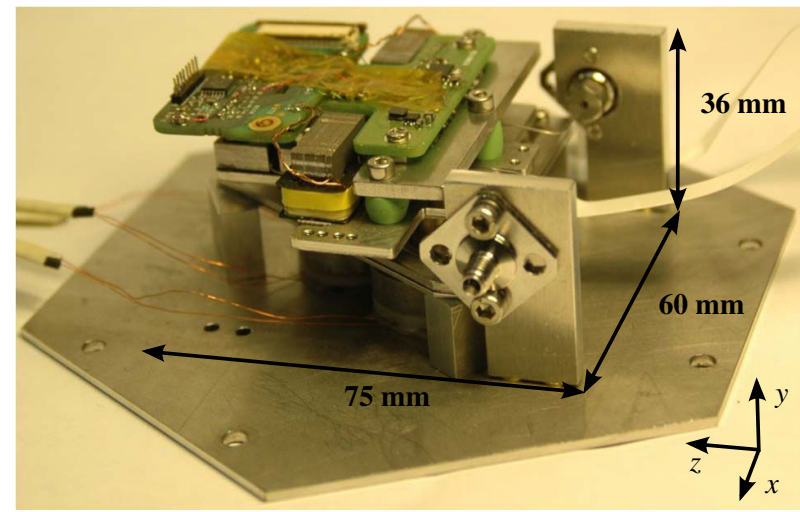

Fig. 4.16 Photo of the experimental setup (Fig.4.13), including the main dimensions.

\subsection{Experimental validation}

In this section the control algorithm of section 4.3 is validated, using an experimental setup, as explained in section 4.4. First the experimental setup and the used identification method are explained. Secondly, the dynamic parameters of the built system are identified. Further, the influence of external vibrations is compared for all presented strategies. Finally, the attenuation of the mass-flow measurement error is shown.

\subsubsection{Experimental setup}

The experimental setup, based on the design of section 4.4 is depicted in Fig. 4.16. Three voice coil actuators are available to apply a force between the base and the stage. The active strategies are implemented using MATLAB XPC Target in combination with an NI-6259 data acquisition card. The real-time system runs with a sample rate of $10 \mathrm{kHz}$.

To validate the system and to measure the sensitivity for external vibrations, the setup is placed on a 6-DOF shaker (Fig. 4.17). The Stewart type shaker platform is controlled by a dSPACE system. Six voice coil actuators are available to apply a disturbance on the platform. Where six acceleration sensors (Endevco 7703A-1000) on the platform are used as reference sensors $\left(\boldsymbol{a}_{0}\right)$. More details of the setup, used as shaker, are presented in Tjepkema [64].

\subsubsection{Identification method}

For the control design an identification of the mechanism designed in section 4.4 is needed. Therefore a Frequency Response Function matrix (FRF) is measured. The identification method is adopted from Wernholt and Gunnarsson [79] to estimate the following system:

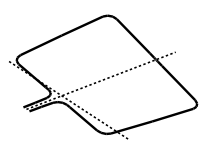




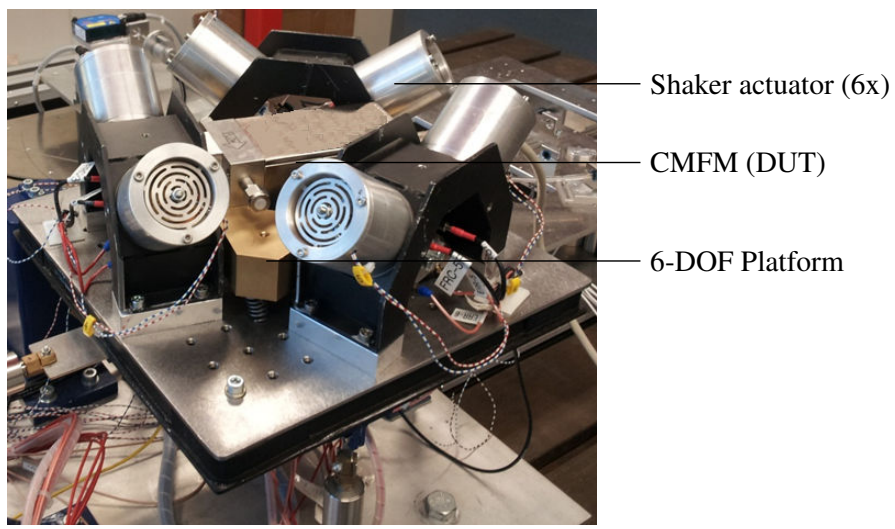

Fig. 4.17 6-DOF Shaker used to apply an external vibration $\boldsymbol{a}_{0}$ on the base plate of the experimental setup (Fig.4.16).

$$
y(t)=\boldsymbol{G} u(t),
$$

where $\boldsymbol{G}$ is a $n_{y} \times n_{u}$ system between the input $u(t) \in \mathbb{R}^{n_{u} \times 1}$ and output $y(t) \in \mathbb{R}^{n_{y} \times 1}$ signals, whereby the noise is assumed to be zero. A Discrete Fourier Transform (DFT) of the system at the frequencies $\omega_{k}$ with sample time $T_{\mathrm{s}}$ reads as:

$$
\boldsymbol{Y}\left(\omega_{k}\right)=\boldsymbol{G}\left(e^{\mathrm{j} \omega_{k} T_{\mathrm{s}}}\right) \boldsymbol{U}\left(\omega_{k}\right),
$$

where $\boldsymbol{G}\left(e^{\mathrm{j} \omega_{k} T_{\mathrm{s}}}\right) \in \mathbb{C}^{n_{y} \times n_{u}}$ is the FRF-matrix and, $\boldsymbol{Y}\left(\omega_{k}\right) \in \mathbb{C}^{n_{y} \times n_{e}}$ and $\boldsymbol{U}\left(\boldsymbol{\omega}_{k}\right) \in \mathbb{C}^{n_{u} \times n_{e}}$ are the DFTs of the input and output signals of $n_{e}$ experiments. To extract the FRF-matrix at least $n_{e} \geq n_{u}$ different experiments are needed. An estimate is given by the $H_{1}$-estimator:

$$
\hat{\boldsymbol{G}}\left(e^{\mathrm{j} \omega_{k} T_{\mathrm{s}}}\right)=\boldsymbol{Y}\left(\omega_{k}\right) \boldsymbol{U}^{\dagger}\left(\omega_{k}\right),
$$

where $\boldsymbol{U}^{\dagger}\left(\omega_{k}\right)$ is the pseudo-inverse of $\boldsymbol{U}\left(\omega_{k}\right)$. The system is excited using orthogonal random phase multi-sine signals to minimise the variance of the FRF estimate [79]. For the identifications a multi-sine with frequencies between 5 and $500 \mathrm{~Hz}$ is used.

\subsubsection{System identification}

The primary and secondary path of the designed mechanism are estimated using the method described in section 4.5.1.1 First the secondary path FRF-matrix $\hat{\boldsymbol{S}}\left(\omega_{k}\right)$ between the actuator input $\boldsymbol{U}$ and the stage sensors $\boldsymbol{a}_{1}$ is estimated. The location of the actuators and sensors results in a MIMO system with interaction between the inputs and the outputs. To use the

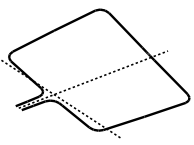


SISO controllers, designed in section 4.3 , we need a diagonal plant:

$$
\boldsymbol{G}_{\mathrm{diag}}(s)=\boldsymbol{T}_{\mathrm{y}} \boldsymbol{G}(s) \boldsymbol{T}_{\mathrm{u}}
$$

Many decoupling procedures have been developed in the past to determine the transformation matrices $\boldsymbol{T}_{\mathrm{y}}$ and $\boldsymbol{T}_{\mathrm{u}}$. A general decoupling transformation is using Dyadic Transfer function Matrices. The method was first introduced by Owens [50]. If $\boldsymbol{G}(s)$ is dyadic, $\boldsymbol{T}_{\mathrm{y}}^{-1}$ can be calculated as the eigenvectors of $\boldsymbol{G}\left(\mathrm{j} \omega_{2}\right) \boldsymbol{G}^{-1}\left(\mathrm{j} \omega_{1}\right)$ and $\boldsymbol{T}_{\mathrm{u}}$ can be calculated as the eigenvectors of $\boldsymbol{G}^{-1}\left(\mathrm{j} \omega_{1}\right) \boldsymbol{G}\left(\mathrm{j} \omega_{2}\right)$, whereby $\omega_{1}$ and $\omega_{2}$ are two frequencies chosen from the FRFmatrix [2]. When the system is not dyadic, the calculated transformation matrices are complex. Real matrices are obtained using an align-method [41], introducing off-diagonal elements in $\boldsymbol{G}_{\text {diag }}(s)$. The decoupling quality strongly depends on the choice of $\omega_{1}$ and $\omega_{2}$ [66]. In the decoupling $\omega_{1}=20 \cdot 2 \pi \mathrm{rad} / \mathrm{s}$ and $\omega_{2}=300 \cdot 2 \pi \mathrm{rad} / \mathrm{s}$ are used, because we want to have proper decoupling at the cross-overs of the open-loop system $S(s) C_{1}(s)$. The calculated transformation matrices for $\hat{\boldsymbol{S}}\left(\omega_{k}\right)$ are:

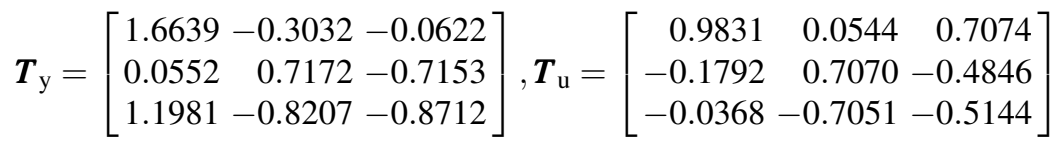

The result of the decoupled plant is depicted in Fig. 4.18 At low frequencies three resonance frequencies around $30 \mathrm{~Hz}$ are clearly visible, these are the three suspension frequencies of the designed stage. At high frequencies an anti- and resonance-frequency of an internal mode around $280 \mathrm{~Hz}$ is visible. Because the system is still co-located, no stability issues are expected. Based on Eq. 4.28 and the geometry, as shown in Fig. 4.16, by approximation the first direction is a translation in $y$-direction, the second a rotation around the $z$-axis and the third a rotation around the $x$-axis.

Secondly, the FRF-matrix $\hat{\boldsymbol{P}}\left(\omega_{k}\right)$ between the base sensors $\boldsymbol{a}_{0}$ and the stage sensors $\boldsymbol{a}_{1}$ is estimated. This is the transmissibility of external vibrations to the stage vibrations. Sufficient vibration isolation of the flow-measurement is achieved when the dominant $y$-translation of the stage in the percussion point is minimised (Chapter 2). The result $\hat{\boldsymbol{T}}\left(\omega_{k}\right)=\boldsymbol{R}_{\mathrm{y}} \hat{\boldsymbol{P}}\left(\omega_{k}\right) \boldsymbol{R}_{0}$ is depicted in Fig. 4.19, whereby $\boldsymbol{R}_{0}$ is the relation between the three translations and three rotations of the base and the $\boldsymbol{a}_{0}$ sensor coordinates. The magnitude in the region of interest is the gained attenuation using vibration isolation. The dominant disturbance directions are a translation in $y$ and $z$-direction. The sensitivity in $z$-direction was not expected, but it is due to the fact that the centre of mass and the centre of compliance are in different points. Therefore, a disturbance in $z$-direction results in tilting of the stage and thus a $y$-translation at the $\boldsymbol{a}_{1}$ sensor positions.

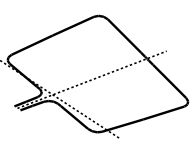



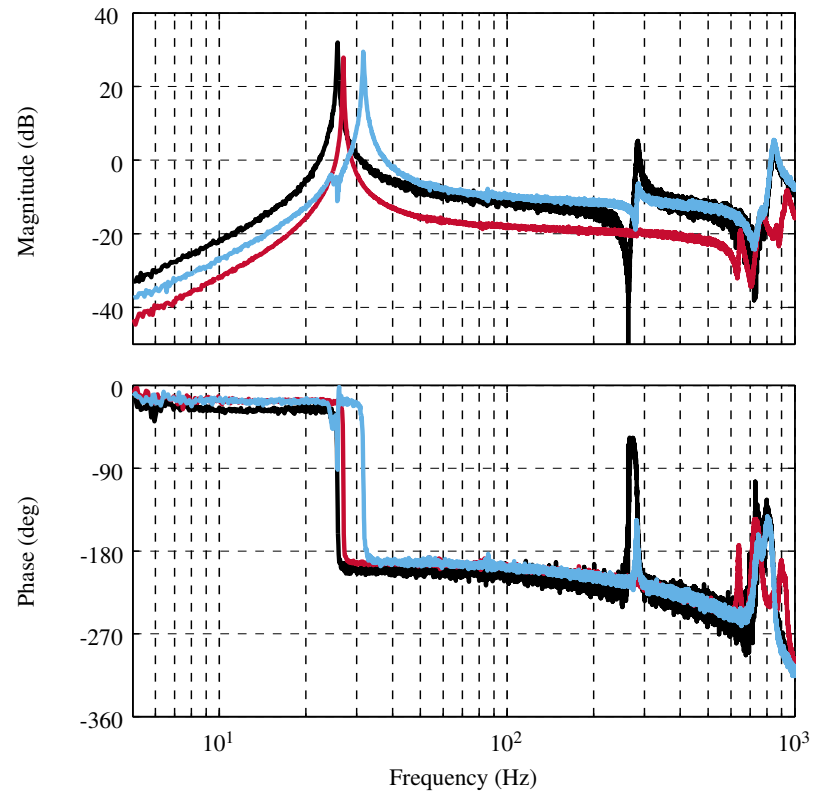

Fig. 4.18 System $\boldsymbol{T}_{\mathrm{y}} \hat{\boldsymbol{S}}\left(\omega_{k}\right) \boldsymbol{T}_{\mathrm{u}}$ identification of the secondary path. Only the diagonal terms are depicted. The three suspension frequencies of the 3-DOF stage at $[25.8,27.0,31.7] \mathrm{Hz}$ are visible. The transfer function $S(s)$ of the model is depicted in Fig. 4.5

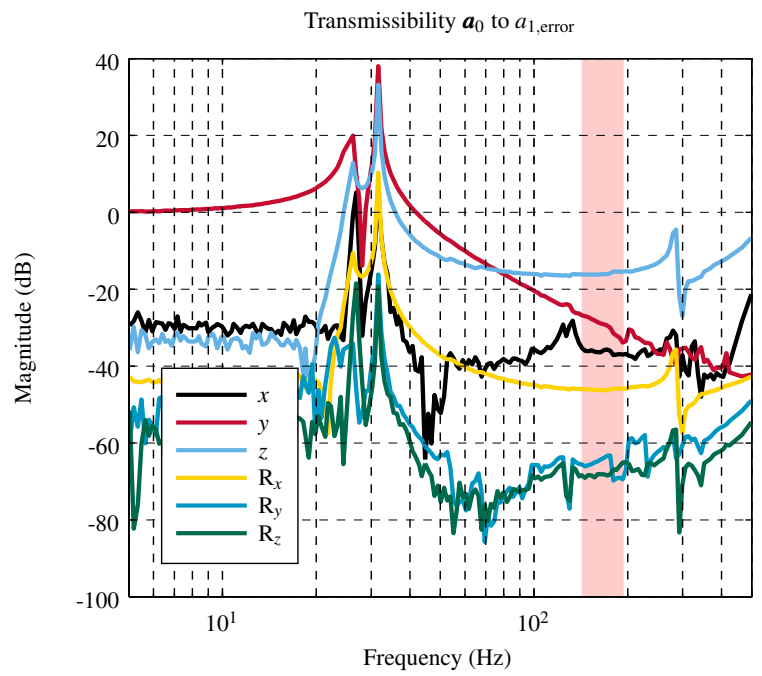

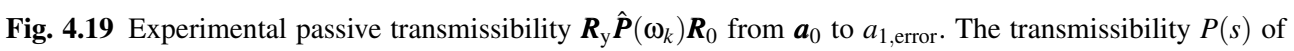
the model is depicted in Fig. 4.5

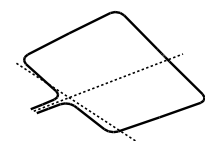




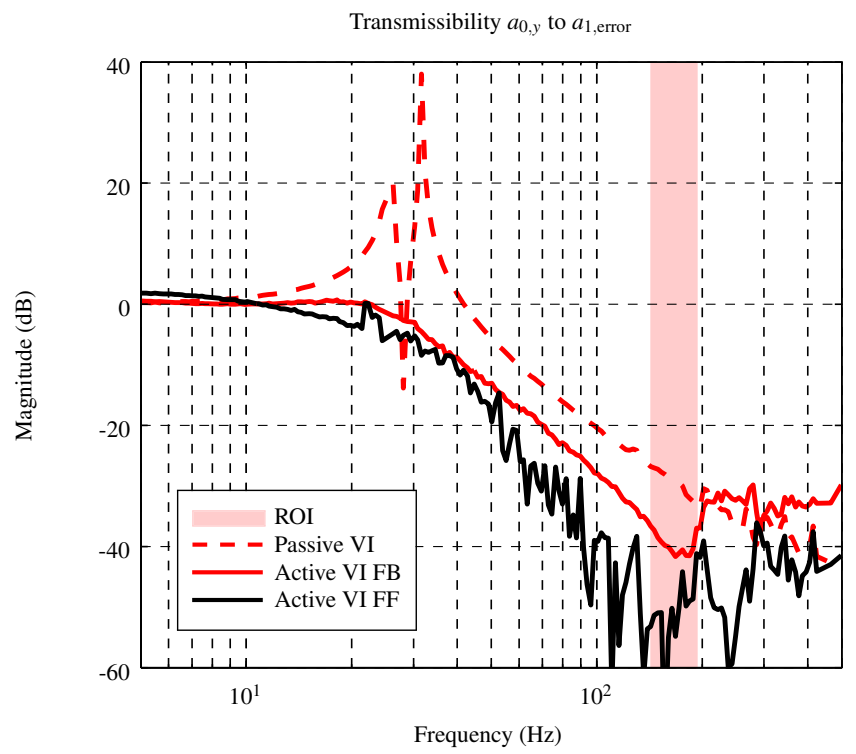

Fig. 4.20 Active SISO transmissibility from $a_{0, y}$ to $a_{1, \text { error }}$. The modelled transmissibility is depicted in Fig. 4.9

\subsubsection{Active vibration isolation}

The achieved passive vibration isolation is limited, as shown in Fig. 4.19. In this subsection the results of active vibration isolation with the controllers, as designed in section 4.3 , are shown. First in one direction, as considered in the 1D model, and thereafter for multiple directions.

\subsubsection{SISO}

First the active vibration isolation strategy is validated with a 1-DOF input disturbance in the dominant $y$-translation direction. The results are shown in Fig. 4.20, To obtain a stable feedback system, addition of notch filters at the frequencies [845, 950, 2190, 2270, 2787] Hz was needed to suppress the higher order dynamics. Attenuation in the region of interest is achieved up to $40 \mathrm{~dB}$ for both strategies. Compared to the passive vibration isolation the active system adds only a minimal extra attenuation. Which is about $10 \mathrm{~dB}$ for both the feedback and feedforward strategy. Outside the region of interest the undamped suspension modes (around $30 \mathrm{~Hz}$ ) is clearly visible. The result is as expect from the model, see Fig.4.9, of more than $40 \mathrm{~dB}$ attenuation. The feedforward result is noisy, because sensor noise makes the FRF estimate noisy. This can be reduced by increasing the level of applied external vibrations $\boldsymbol{a}_{0}$.

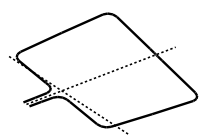




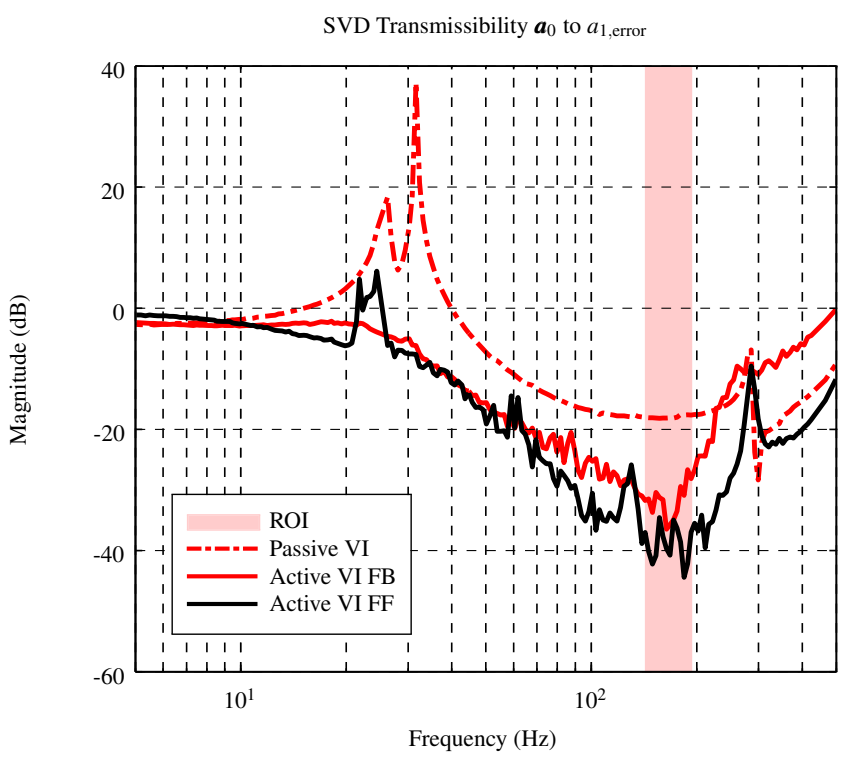

Fig. 4.21 Active SVD transmissibility from $\boldsymbol{a}_{0}$ to $a_{1, \text { error }}$. The modelled transmissibility is depicted in Fig.4.15

\subsubsection{MIMO}

There is not one dominant direction, but there are multiple directions, as shown in Fig.4.19 The same figure can be shown for the active vibration strategies, but there are too many lines for a clear result. Therefore only the dominant direction is shown. This is realized by a Singular Value Decomposition (SVD) for each frequency. The largest singular value gives the influence for the dominant direction for that certain frequency. In any other direction the vibration isolation is better. The result of passive vibration isolation can now be summarised in one line, as shown in shown in Fig. 4.21, which is approximately the largest point in Fig. 4.19 for each frequency for the passive isolation.

First the active strategies, presented in section 4.3 , are adapted to the $3 \times 3$ MIMO system of the 3-DOF stage. In Fig. 4.18 three decoupled transfer functions are depicted, all three are used as a SISO system. For this validated secondary path, the gains of the feedback controllers are calculated, using Eq. 4.8. Note that also for the MIMO feedback, the added notch filters are needed for a stable system.

The MIMO formulation of the feedforward strategy is presented in subsection 4.3.2.1 For feedforward the weights are made adaptive to achieve maximum attenuation, see subsection 4.3.2. As error signal the combined stage acceleration $a_{1, \text { error }}$ is used. In Fig. 4.19 is shown that the two dominant disturbances are $\boldsymbol{a}_{0}$-accelerations in $y z$-direction. Both measured accelerations are used as reference signals. For the $y$-disturbance the primary path and secondary path (Fig. 4.19 and 4.18) are according to the model. Therefore, the controller

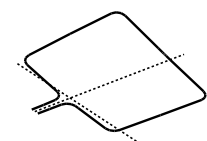


structure, given in Eq. 4.10, is used. For three actuators and one error signal, this results in $3 \times 3=9$ weights.

For a $z$-disturbance the primary path has a different structure. In the region of interest the slope is $0 \mathrm{~dB} /$ decade in stead of $-40 \mathrm{~dB} /$ decade (see Fig.4.19). This changes the optimal controller structure $C_{\mathrm{FF}}(s)$ for a $a_{0, z}$-disturbance. The ideal controller structure for a $z$-disturbance is:

$$
C_{\mathrm{FF}, z}(s)=-g \frac{d+k}{s^{2}} \cdot \frac{s+\omega_{\text {ind }}}{\omega_{\text {ind }}}=\left[-k-\left(d+\frac{k}{\omega_{\text {ind }}}\right)-\frac{d}{\omega_{\text {ind }}}\right]\left[\begin{array}{c}
1 \\
s \\
s^{2}
\end{array}\right],
$$

where the stiffness $k$, damping $d$ and gain $g$ can be determined by the system identification (Section 4.5.2). The main difference between Eq. 4.10 and 4.29 is the filter $F(s)$. For a $z-$ disturbance a IIR different filter, compared to Eq.4.17, is proposed:

$$
\boldsymbol{F}_{\mathrm{Z}}(z)=\left[1 \frac{1}{T_{\mathrm{s}} \omega_{\mathrm{x}}(z-1)}\right] \frac{\alpha_{2}^{2} T_{\mathrm{s}}^{2}}{\left(z-\left(1-\alpha_{2} T_{\mathrm{S}}\right)\right)^{2}}
$$

where the differentiator has a cut-off frequency at $\alpha_{2}=500 \cdot 2 \pi \mathrm{rad} / \mathrm{s}$ to limit the infinite gain and the double integrator is omitted, because of its high gain for the higher frequencies. For three actuators and one error signal, this results in $3 \times 2=6$ extra weights.

Summarising, there are two reference signals and three actuators, resulting in a $3 \times 2$ MIMO controller with 15 weights in total. The settling of the weights is depicted in Fig. 4.22 for $\bar{\mu}=$ 0.01 and $\varepsilon=1$. The weights are not a real estimate for the stiffness, damping and induction pool, because these are adapted such that the influence of external vibrations is minimal in the region of interest in the presence of the residual noise shape filter $N(s)$.

In Fig. 4.21 the MIMO results of the active vibration strategies are shown. The results are less than expected from the 1D model, because of the influence of a disturbance in $z$-direction. For both strategies a minimum of $30 \mathrm{~dB}$ attenuation is achieved, for each direction of the input disturbance. The feedforward strategy results in a lower gain at the higher frequencies, while using the feedback strategy results in a magnification compared to the passive system.

\subsubsection{Measurement error}

So far the reduction from the base to the platform acceleration is considered. However, active vibration isolation is integrated in a CMFM to reduce the influence of external vibrations on the measurement value. The experimental setup is shown in Fig. 4.16, In this section the newly achieved performance is determined for a disturbance in the dominant $y$-direction. In Fig. 4.23 the measurement signal is compared for the different configurations with an applied broadband $y$-disturbance of $4 \mathrm{e}-6 g^{2} / \mathrm{Hz}$ between 5 and $500 \mathrm{~Hz}$. The RMS values of those experiments are given in Table 4.1. Attenuation of the RMS measurement error is $36 \mathrm{~dB}$ and is achieved for both the feedback and feedforward strategy. Whereby the attenuation is reduced to the noise level of the measurement value without a vibrational disturbance.

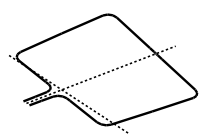




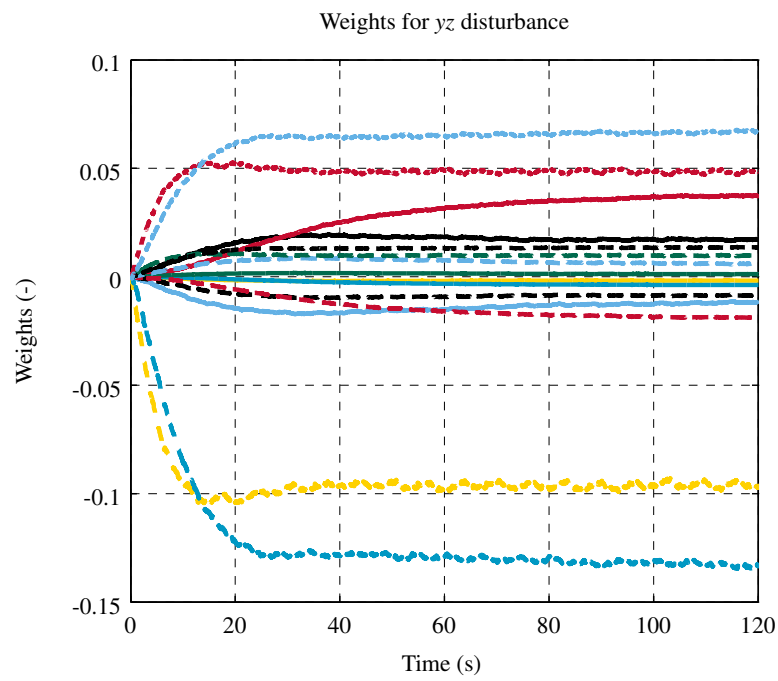

Fig. 4.22 Time plot of the settling of the $(3 \times 3+3 \times 2=15)$ weights for a $y z$-disturbance adaptive feedforward.

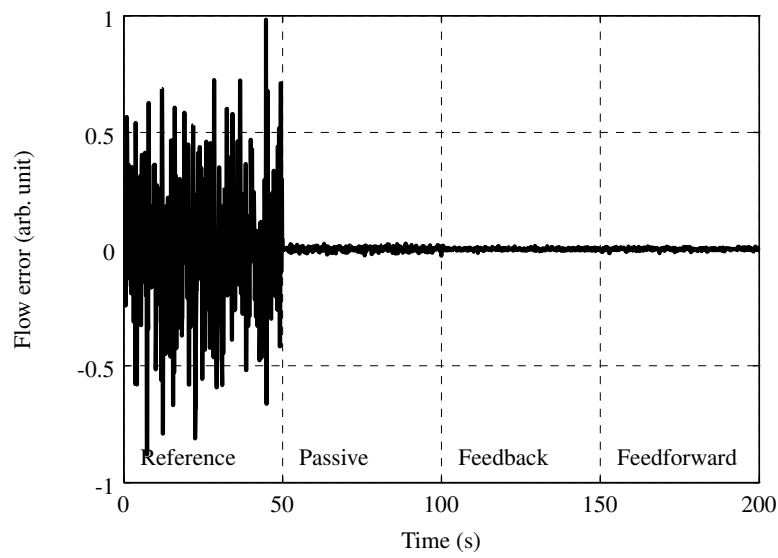

Fig. 4.23 Time domain flow error (Normalised) - 50s of the reference, passive and active (feedback and feedforward) system for $a_{y}$ white broadband disturbance level of $4 \mathrm{e}-6 g^{2} / \mathrm{Hz}$ between 5 and $500 \mathrm{~Hz}$.

In Fig. 4.11(a) the influence at $175 \mathrm{~Hz}$ is depicted for transmissibility $T_{y_{\text {cor }}, a_{1}}$. The applied excitation level of $2 \mathrm{e} 3 \mu \mathrm{g} / \sqrt{\mathrm{Hz}}$ contributes $2.5 \mathrm{e}-8 \mathrm{~m} / \sqrt{\mathrm{Hz}}$ to the Coriolis-motion and by the $40 \mathrm{~dB}$ reduction this is reduced to $2.5 \mathrm{e}-10 \mathrm{~m} / \sqrt{\mathrm{Hz}}$. However, the noise due to the displacement sensors is $3.16 \mathrm{e}-10 \mathrm{~m} / \sqrt{\mathrm{Hz}}$, limiting the reduction to $(3.16 \mathrm{e}-10) /(2.5 \mathrm{e}-8)=$ $-38 \mathrm{~dB}$, which is close to the observed reduction. So the achieved attenuation, is limited by the noise level of the tube displacement sensor. For the shaker setup (Fig. 4.17) it was 
not possible to increase the excitation level further, so the expected attenuation of more than $40 \mathrm{~dB}$, as presented in section 4.5.3.1, could not be verified.

Table 4.1 RMS flow measurement error values for a broadband disturbance of $4 \mathrm{e}-6 g^{2} / \mathrm{Hz}=2 \mathrm{e} 3 \mu \mathrm{g} / \sqrt{\mathrm{Hz}}$

\begin{tabular}{lcc}
\hline & RMS error (arb. unit) & Attenuation $(\mathrm{dB})$ \\
\hline no Dist. & 0.0045 & - \\
Reference & 0.3043 & - \\
Passive & 0.0096 & -30.0 \\
Feedback & 0.0044 & -36.8 \\
Feedforward & 0.0049 & -35.9 \\
\hline
\end{tabular}

\subsection{Discussion}

With vibration isolation a reduction of the influence of external vibrations is achieved on the transmissibility and on the measurement value. Several improvements can be made to obtain the attenuation expected from the 1D model and even more attenuation can be obtained. First the design of the compliantly suspended stage should be improved. As shown in Fig. 4.19, the passive suspension is not giving the attenuation belonging to the suspension frequency of $30 \mathrm{~Hz}$, because a translation causes an unexpected tilt of the stage. This can be improved by placing the centre of mass in the centre of compliance, or by making the stage stiff in rotation direction. The flexible suspension in the $y$-direction still guarantees the attenuation for rotational disturbances.

To lower the noise level of the measurement value further, tube-displacement sensors with a lower noise level should be used. This displacement sensors can also be used in the feedforward strategy as error sensors instead of the acceleration sensors $a_{1}$.

Further the feedforward controller structure should be changed, if the influence of a $z-$ disturbance cannot be minimised in the design. As shown in the validation of the system in subsection 4.5.2, the structure of the secondary path is not as in the model (Eq.4.6). Therefore the optimal feedforward controller (Eq.4.9) is different.

The performance of active vibration isolation is always dependent on the noise level of the sensors. In subsection 4.3.3.1 the effect of sensor noise on the stage acceleration $a_{1}$ is given. Showing that for the feedback strategy compared to the feedforward strategy, sensors with a lower noise floor are needed. The performance of the adaptive feedforward is also dependent on noise of the error sensor. In our validation the stage acceleration sensors are used and the weights are tuned such that the stage accelerations approach the noise level of the stage accelerometers. For the level of applied external disturbances, this maximises the attenuation at $175 \mathrm{~Hz}$ to about $50 \mathrm{~dB}$. To increase the attenuation of the adaptive feedforward strategy, the noise level of the error sensors should be decreased. Alternatively and even better, the tube displacement $y_{\text {cor }}$ can be used as error sensor.

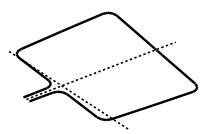




\subsection{Conclusions}

Vibration isolation of more than $40 \mathrm{~dB}$ is achieved for a Coriolis Mass-Flow Meter (CMFM) with integrated Active Vibration Isolation. The measurement stage is passively suspended at $30 \mathrm{~Hz}$ in the 3 out-of-plane directions and active vibration isolation is added to increase the attenuation. In this paper the modelled system and controller design are presented. Based on the model an on-scale proof of principle is built and validated in multi-DOF. Active vibration isolation increases the attenuation of 10 to $20 \mathrm{~dB}$ at $175 \mathrm{~Hz}$ in addition to the $30 \mathrm{~dB}$ attenuation obtained by a $30 \mathrm{~Hz}$ passive vibration isolation stage.

A feedback and feedforward strategy are compared and both result in a large attenuation of the influence of external vibrations on the measurement value. A filtered-reference leastmean-square (FxLMS) adaptive scheme is used to determine the optimal feedforward controller parameters to minimise a squared error signal; the motion of the measurement stage. The performance of the feedback control, based on acceleration feedback, is limited due to issues to guarantee stability and therefore the attenuation is less, compared to the feedforward strategy. The overall active vibration isolation performance is limited by the noise levels of the used sensors. Even lower noise sensors means more costly sensors, but an increased performance. The results obtained in this paper can be used to find the balance between proper sensors and vibration isolation performance.

The designed stage had more than one dominant direction of influence of external vibrations. This will be resolved in future research by placing the centre of mass in the centre of compliance, or by changing the number of degrees-of-freedom of the suspended stage. 


\title{
Chapter 5 \\ Vibration isolation by compliant sensor mounting
}

\begin{abstract}
In this paper a vibration isolated design of a Coriolis Mass-Flow Meter (CMFM) is proposed, by introducing a compliant connection between the casing and the tube displacement sensors, with the objective to obtain a relative displacement measurement of the fluid conveying tube, dependent on the tube actuation and mass-flow, but independent of external vibrations. The transfer from external vibrations to the relative displacement measurement is analysed and the design is optimised to minimise this transfer. The influence of external vibrations on a compliant sensor element and the tube are made equal by tuning the resonance frequency and damping of the compliant sensor element and therefore the influence on the relative displacement measurement is minimised. The optimal tuning of the parameters is done actively by acceleration feedback. Based on simulation results, a prototype is built and validated. The validated design shows more than $24 \mathrm{~dB}$ reduction of the influence of external vibrations on the mass-flow measurement value of a CMFM, without affecting the sensitivity for mass-flow.
\end{abstract}

This chapter is preprinted from: L. van de Ridder, W. B. J. Hakvoort, J. van Dijk, J. C. Lötters, and A. de Boer. Vibration isolation by an actively compliantly mounted sensor applied to a coriolis mass-flow meter. 2015. Submitted to Dynamic Systems, Measurement and Control (ASME)

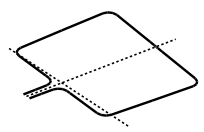




\subsection{Introduction}

Vibration isolation is extremely important in high precision machines for surface treatments (e.g. lithography machines) or measurements (e.g. scanning electron microscopes) that should be accurate to nanometre level. Vibration isolation can be achieved with passive isolators. Passive isolation consist of several stages of mass-spring-damper systems between the floor and the casing of a machine [53], the parameters are adjusted to achieve high-frequency attenuation, which is appropriate for many applications. The better the vibration isolation system the better the decoupling of the internal measurement system from any environmental disturbances. However, the performance of passive isolation can be limited due to a minimal required suspension stiffness. An alternative approach is to apply active vibration isolation control [77, 52]. In this paper an active vibration isolated measurement system applied to a Coriolis Mass-Flow Meter (CMFM) is presented.

A CMFM is an active device based on the Coriolis force principle for direct mass-flow measurements independent of fluid properties [1, 78]. The CMFM contains a fluid conveying tube, which is actuated to oscillate in resonance with a low amplitude. A fluid flow in the vibrating tube induces Coriolis forces, proportional to the mass-flow, which affect the modeshape of the actuation mode. Measuring the tube displacements allows measuring the massflow. Besides an effect of the mass-flow on the modeshape, external vibrations can introduce motions that cannot be distinguished from the Coriolis force induced motion [13, 14], thus introducing a measurement error. The influence of external vibrations on the measurement value can be estimated quantitatively as shown in Chapter 2

It is necessary to incorporate a a balancing mechanism for flow meters, which allows accurate measurements for various process conditions and changing fluid densities [1]. There is only attenuation needed in a relative small range around the actuation frequency (Chapter 2), vibration isolation solutions can be applied, without interfering with the accurate measurement of the displacements of the internal actuation modeshape.

In this paper a novel design of a CMFM is proposed, by introducing a compliant connection between the casing and the tube displacement sensors with the objective to obtain a relative displacement measurement of the tube, dependent on the tube actuation and mass-flow, though independent of external vibrations. The design is analysed to optimise the transfer function from the external vibrations to the relative displacement measurement. The influence of external vibrations on the compliant sensor element and the tube are made equal by actively tuning the resonance frequency and damping of the compliant sensor element and thereby the influence on the relative displacement measurement is minimised. Based on simulation results, a prototype is built and validated. This paper is an extension of [71]: (i) the effects of changing the internal dynamics due to a fluid-density change are addressed in detail, (ii) the optimal suspension frequency of the compliant sensor element is determined and (iii) the attenuation is maximised by active tuning of the compliant sensor element resonance frequency.

In the next section the performance criterion is given for the level of vibration isolation. In section 5.3, the novel design is introduced and the transmissibility from external vibrations to an internal deformation is derived, based on a model for a reference instrument and for the new design in the passive and active configuration. In section $5.4 \mathrm{a}$ mechanical design is

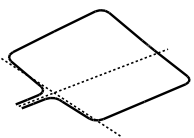




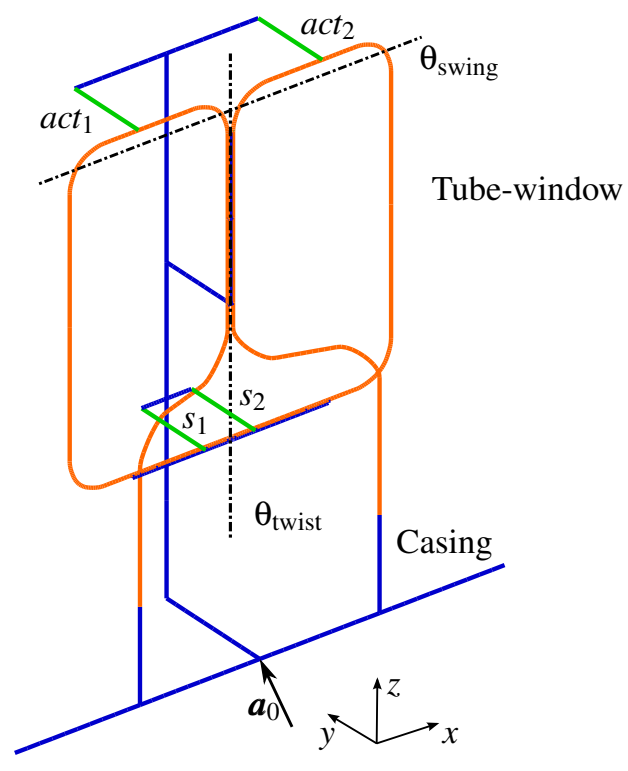

Fig. 5.1 Multibody model of the CMFM, adopted from Chapter 2 The tube-window is actuated, by means of $a c t_{1}$ and $a c t_{2}$, to oscillate around the $\theta_{\text {twist }}$-axis. On the casing, external vibrations $\boldsymbol{a}_{0}$ are applied. Sensors $\left(s_{1}\right.$ and $s_{2}$ ) measure the relative tube displacements, affected by the actuation, a mass-flow and external vibrations.

presented, based on the concept presented in section 5.3 The design is validated experimentally in section 5.5. Results are discussed in section 5.6 and the conclusions of this paper are presented in the final section 5.7

\subsection{Performance criteria}

Prior to the design and analysis of a vibration isolator, a performance criterion is needed. In this section a definition is given, taking into account how external vibrations affect the mass-flow measurement of a CMFM.

A functional model of a CMFM is presented in various patents [45, 46]. Of this instrument a flexible multibody model (see Chapter 2) is made to derive the influence of external vibrations on the measurement value, using [31, 48]. An illustration of the model is given in Fig. 5.1. The model consists of a rigid casing and a flexible tube-window, conveying the fluid flow, which is actuated by two Lorentz actuators $a c t_{1}$ and $a c t_{2}$. The displacement of the tube-window is measured by two optical displacements sensors $s_{1}$ and $s_{2}$ [46]. On the casing an input vector $\boldsymbol{a}_{0}$, consisting of three translation and three rotational casing movements, is imposed.

A Lorentz actuator is used to oscillate the tube-window around the $\theta_{\text {twist }}$-axis in its resonance frequency $\omega_{\text {act }}$. In the model the measured actuation displacement is the difference

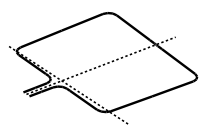


between the two sensor signals, located at equal distance $r$ of the rotation axis:

$$
y_{\mathrm{act}}=r \cdot \theta_{\mathrm{twist}}=\frac{1}{2}\left(s_{1}-s_{2}\right)
$$

Due to a rotating reference frame, the tube, and a moving mass, the fluid, there is a Coriolis force. This force is acting on the tube-window and is proportional to the angular velocity and the mass-flow $\dot{\Phi}_{\mathrm{m}}$ through the tube:

$$
F_{\text {cor }}=-2 L_{\text {eff }} \cdot \dot{\theta}_{\text {twist }} \times \dot{\Phi}_{\mathrm{m}}
$$

where $L_{\mathrm{eff}}$ is the effective length of the tube. The force $F_{\text {cor }}$ results in a rotation of the tubewindow around the $\theta_{\text {swing }}$-axis (see Fig. 5.1). A rotation around this axis results in a displacement at the location of the sensors. This measured displacement is defined as a Coriolis displacement:

$$
y_{\text {cor }}=\frac{1}{2}\left(s_{1}+s_{2}\right)
$$

The Coriolis displacement, due to a fluid flow, is a harmonic with the same frequency $\omega_{\text {act }}$ as the actuation displacement, though $90^{\circ}$ out of phase. This is due to the velocity dependency of the force, expressed by Eq. 5.2 A phase difference between the two harmonic sensor signals $s_{1}$ and $s_{2}$, which are shifted $180^{\circ}$ nominally, can be approximated by:

$$
\Delta \phi \approx 2\left|\frac{s_{1}+s_{2}}{s_{1}-s_{2}}\right|=2\left|\frac{y_{\text {cor }}}{y_{\text {act }}}\right|
$$

The actuation displacement $y_{\text {act }}$ is controlled with an internal actuator to be constant and the Coriolis displacement $y_{\text {cor }}$ is due to a mass-flow through the instrument, resulting in a phase difference $\Delta \phi$ which is proportional to the mass-flow $\dot{\Phi}_{\mathrm{m}}$.

Thus, the mass-flow measurement value is obtained from the phase difference between two harmonics, which are the tube displacements measured by two sensors. The frequency of the harmonic is known to be the actuation frequency of the tube window. Only the phase difference of this particular harmonic is needed for obtaining the mass-flow measurement. The phase difference is acquired by phase demodulation, which can be seen as a band pass filter on the Coriolis displacement signal around the actuation frequency (see Chapter 2), of which the width is dependent on the required response time of the measurement value due to a flow rate change.

A Coriolis displacement $y_{\text {cor }}$ that is not related to a mass-flow through the instrument, but for instance as a result of external vibrations, can result in a measurement error. The effect of external vibrations is undesirable, therefore its transmissibility should be minimised.

In this paper the performance criterion is defined as: minimisation of the transmissibility $T_{y_{\text {cor }}, \boldsymbol{a}_{0}}(s)$ of external vibrations $\boldsymbol{a}_{0}(s)$ to an internal deformation $y_{\text {cor }}(s)$ around the actuation frequency $\omega_{\text {act }}$ without affecting the transmissibility $T_{y_{\text {cor }}, F_{\text {cor }}}(s)$ of a Coriolis force $F_{\text {cor }}(s)$ to the internal deformation $y_{\mathrm{cor}}(s)$. The attenuation is defined as the ratio by which $T_{y_{\mathrm{cor}}, a_{0}}(s)$ is reduced.

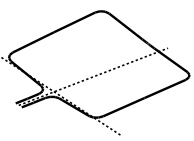




\subsection{Conceptual design}

In this section a solution is proposed to minimise the transmissibility $T_{y_{\mathrm{cor}}, a_{0}}(s)$, which is the performance criterion, explained in the previous section. First, the transmissibility is derived for the reference system and for the new design. In the second subsection the main design parameters for minimising the transmissibility are presented. In the final subsection the design is extended with an active part to actively optimise the transmissiblity.

\subsubsection{Simplified model}

Earlier modelling results indicate one dominant direction for the mass-flow measurement, a translation in the direction out of the tube-window plane (See Chapter 2). Therefore the effect of external vibrations and the mass-flow are modelled in a simple and elegant manner by a simple mass-spring model, as depicted in Fig. 5.2. The tube parameter 11 are modelled with the modal mass $m_{2}=1.133 e-4 \mathrm{~kg}$, damping $d_{2}=6.836 e-5 \mathrm{Ns} / \mathrm{m}$ and stiffness $k_{2}=$ $41.25 \mathrm{~N} / \mathrm{m}$ and there are two inputs, an external vibration $a_{y}=\ddot{y}_{0}$ and a Coriolis force $F_{\text {cor }}$ and one output $y_{\text {cor }}$, which is a relative displacement measurement between the mass $m_{2}$ and the casing. The modelled mode has a resonance frequency $\omega_{\text {cor }}=\sqrt{k_{2} / m_{2}}=96.0 \cdot 2 \pi \mathrm{rad} / \mathrm{s}$. The Coriolis force, due to a mass-flow, is a harmonic with the frequency $\omega_{\text {act }}=170.0 \cdot 2 \pi \mathrm{rad} / \mathrm{s}$. The modal model reduction of the system is presented in Chapter 2 .

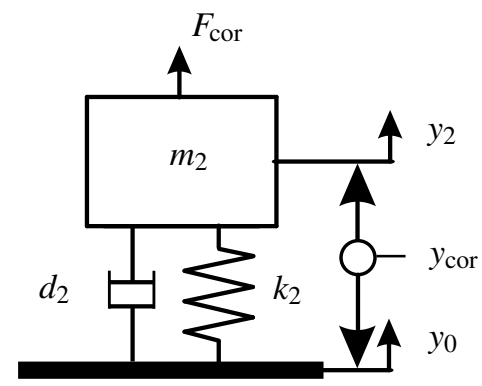

Fig. 5.2 1D CMFM model, representing the Coriolis mode. The Coriolis displacement $y_{\text {cor }}$ is affected by the Coriolis force $F_{\text {cor }}$ and an external displacement $y_{0}$.

According to this model (Fig. 5.2) of the reference system, the relative displacement in the Laplace $s$-domain is equal to:

$$
\begin{aligned}
y_{\mathrm{cor}}(s) & =y_{2}(s)-y_{0}(s) \\
& =T_{y_{\mathrm{cor}}, F_{\mathrm{cor}}}(s) F_{\mathrm{cor}}(s)+T_{y_{\mathrm{cor}}, a_{y}}(s) a_{y}(s) \\
& =\frac{1}{m_{2} s^{2}+d_{2} s+k_{2}} F_{\mathrm{cor}}(s)+\frac{-1}{s^{2}+\frac{d_{2}}{m_{2}} s+\frac{k_{2}}{m_{2}}} a_{y}(s)
\end{aligned}
$$

\footnotetext{
${ }^{1}$ Note that the tube geometry in this chapter is different than in Chapter 2 and therefore the presented resonance frequencies are higher.
} 
This expression shows that the displacement is indeed dependent on the Coriolis Force and the external vibration $a_{y}$. The influence is dependent on the magnitude of the disturbance, the frequency and the physical parameters of the system. Those physical parameters cannot be changed without changing the sensitivity for a mass-flow.

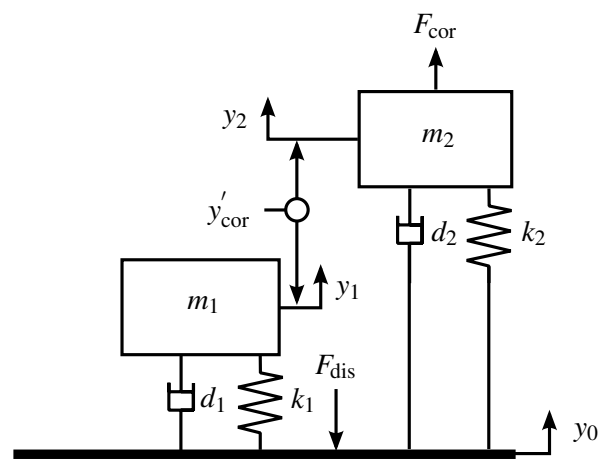

Fig. 5.3 1D CMFM CMS model. Compared to the CMFM model (Fig. 5.2) the measured Coriolis displacement is relative to the flexibly suspended mass $m_{1}$.

Table 5.1 Modal parameters of CMFM model for two different fluid densities $\rho$

\begin{tabular}{rcccccc}
\hline$\rho\left(\mathrm{kg} / \mathrm{m}^{3}\right)$ & $g_{1}(-)$ & $g_{2}(-)$ & $\omega_{\text {cor }, 1}(\mathrm{rad} / \mathrm{s})$ & $\omega_{\text {cor }, 2}(\mathrm{rad} / \mathrm{s})$ & $\omega_{\text {act }}(\mathrm{rad} / \mathrm{s})$ & $Q(-)$ \\
\hline $1($ Air $)$ & -1.133 & 0.2107 & $96.03 \cdot 2 \pi$ & $378.3 \cdot 2 \pi$ & $170.0 \cdot 2 \pi$ & 1000 \\
$998($ Water $)$ & -1.137 & 0.2153 & $91.35 \cdot 2 \pi$ & $356.1 \cdot 2 \pi$ & $160.2 \cdot 2 \pi$ & 1000 \\
\hline
\end{tabular}

To increase vibration isolation, without changing the mass-flow sensitivity, it is proposed to use a compliantly mounted sensor (CMS). The model is shown in Fig. 5.3. The relative displacement measurement is no longer between the casing and the internal mass $m_{2}$, but between an extra mass $m_{1}$ and again $m_{2}$. There is a certain stiffness $k_{1}$ and damping $d_{1}$ between the new mass and the casing. In this model the new relative displacement is equal to:

$$
\begin{aligned}
y_{\mathrm{cor}}^{\prime}(s) & =y_{2}(s)-y_{1}(s)=y_{\mathrm{cor}}(s)-q_{1}(s)= \\
& =T_{y_{\mathrm{cor}}, F_{\mathrm{cor}}}(s) F_{\mathrm{cor}}(s)+T_{y_{\mathrm{cor}}, a_{y}}(s) a_{y}(s)-T_{q_{1}, a_{y}}(s) a_{y}(s) \\
& =\frac{1}{m_{2} s^{2}+d_{2} s+k_{2}} F_{\mathrm{cor}}(s) \\
& +\frac{\left(\frac{d_{2}}{m_{2}}-\frac{d_{1}}{m_{1}}\right) s+\left(\frac{k_{2}}{m_{2}}-\frac{k_{1}}{m_{1}}\right)}{\left(s^{2}+\frac{d_{1}}{m_{1}} s+\frac{k_{1}}{m_{1}}\right)\left(s^{2}+\frac{d_{2}}{m_{2}} s+\frac{k_{2}}{m_{2}}\right)} a_{y}(s)
\end{aligned}
$$

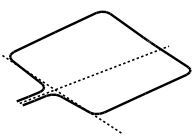


where $q_{1}(s)=y_{1}(s)-y_{0}(s)$. The sensitivity for flow is equal, but the transmissibility of external vibrations to the Coriolis displacement is dependent on the newly introduced parameters. By choosing the mass, damping and stiffness of the compliantly mounted sensor, the influence of the external vibrations $a_{y}$ can be minimised. An optimal result, according to Eq. 5.6, can be achieved when the following conditions are met:

$$
d_{1}=\frac{m_{1}}{m_{2}} d_{2}, \quad k_{1}=\frac{m_{1}}{m_{2}} k_{2}
$$

This is achieved when the resonance frequency $(\omega=\sqrt{\mathrm{k} / \mathrm{m}})$ and damping ratio $(\zeta=d / 2 \sqrt{\mathrm{km}})$ are equal for the internal mode of the tube-window and the compliantly mounted sensor.

\subsubsection{Design parameters}

In this section, the choice of the design parameters $\left(m_{1}, d_{1}\right.$ and $\left.k_{1}\right)$ of the CMS are elaborated. The result, whereby $\omega$ and $\zeta$ are equal for the tube and the internal mode, is depicted as dashed line in Fig. 5.4 whereby the transfer function $T_{y_{\text {cor }}, a_{y}}$ of the model depicted in Fig. 5.1]is used. The use of those parameters does not result in a zero transmissibility, because the simplified mass-spring-damper model (Fig. 5.2) does not represent the higher order dynamics of the tube-window. In the region of interest, this transmissibility can be approximated by adding the next mode in the sensitive direction:

$$
\begin{aligned}
T_{y_{\mathrm{cor}}^{\prime}, a_{y}}(s) & =T_{y_{\mathrm{cor}, a_{y}}(s)-T_{q_{1}, a_{y}}(s)} \\
& \approx \frac{g_{1}}{s^{2}+\frac{1}{Q} \omega_{\mathrm{cor}, 1} s+\omega_{\mathrm{cor}, 1}^{2}}+\frac{g_{2}}{s^{2}+\frac{1}{Q} \omega_{\mathrm{cor}, 2} s+\omega_{\mathrm{cor}, 2}^{2}} \\
& -\frac{-1}{s^{2}+\frac{d_{1}}{m_{1}} s+\frac{k_{1}}{m_{1}}}
\end{aligned}
$$

which is a function of the modal parameters of the CMFM-tube and of the compliantly mounted sensor properties. In Table 5.1 the modal parameters of the CMFM tube model are given.

Evaluating Eq. 5.8 shows that the parameters, given in Eq. 5.7 do not minimise the transmissibility $T_{y_{\text {cor }}^{\prime}, a_{y}}(s)$ in the region of interest. Based on Eq. 5.8, two design parameters are defined: the resonance frequency $\omega_{\mathrm{CMS}}=\sqrt{\frac{k_{1}}{m_{1}}}$ and the damping ratio $\zeta_{\mathrm{CMS}}=\frac{d_{1}}{2 \sqrt{k_{1} m_{1}}}$ of the CMS. In Fig. 5.5 the attenuation of the transmissibility $T_{y_{\text {cor }}^{\prime}, a_{y}}(s)$ at the actuation frequency, as function of the variation of those parameters is depicted. The attenuation is defined as:

$$
\text { Attenuation }(\mathrm{dB})=20 \cdot \log _{10}\left(\left|\frac{T_{y_{\mathrm{cor}}^{\prime}, a_{y}}\left(\mathrm{i} \omega_{\mathrm{act}}\right)}{T_{y_{\mathrm{cor}}, a_{y}}\left(\mathrm{i} \omega_{\mathrm{act}}\right)}\right|\right)
$$

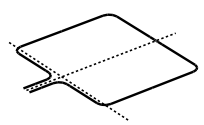




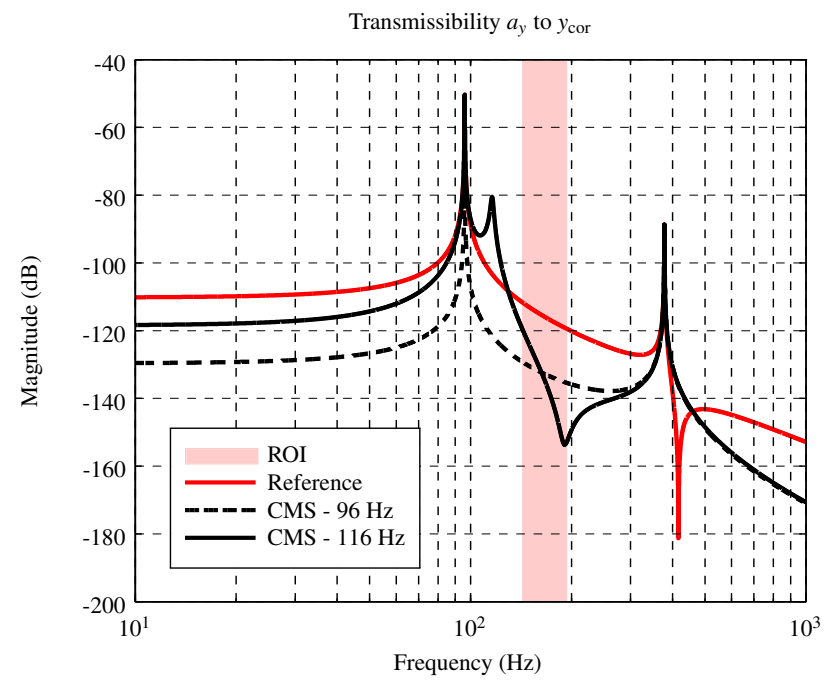

Fig. 5.4 Transmissibility from external vibrations $a_{y}$ to the Coriolis displacement $y_{\text {cor }}$ of the reference system and the CMS system (Eq. 5.8). The CMS transmissibility is depicted for the stiffness and damping parameters according to Eq. 5.7 and for $\omega_{\mathrm{CMS}}=116 \cdot 2 \pi \mathrm{rad} / \mathrm{s}$ and $\zeta_{\mathrm{CMS}}=0.01$. The region of interest is a $25 \mathrm{~Hz}$ band around the actuation frequency $\omega_{\text {act }}$.

From Fig. 5.5 can be concluded that the CMS resonance frequency $\omega_{\mathrm{CMS}}$ should be about $15 \mathrm{~Hz}$ higher than the Coriolis frequency $\omega_{\mathrm{cor}, 1}$ and that the damping should be minimal. In Fig. 5.4, also the transmissibility $T_{y_{\mathrm{cor}}^{\prime}, a_{y}}(s)$ is depicted for $\omega_{\mathrm{CMS}}=116 \cdot 2 \pi \mathrm{rad} / \mathrm{s}$ and $\zeta_{\mathrm{CMS}}=$ 0.01 . It clearly shows the newly introduced resonance frequency, but also an anti-resonance in the region of interest. The appearance of this anti-resonance is used to reduce the influence of external vibrations in the region of interest.

For example, in case the fluid medium is air, tuning the resonance frequency of the CMS to $110 \mathrm{~Hz}$ and minimise the damping, results in an attenuation of more than $30 \mathrm{~dB}$ around the actuation frequency.

\subsubsection{Actively compliantly mounted sensor}

The maximum attenuation is not only dependent on the suspension frequency $\omega_{\mathrm{CMS}}$, it is also a function of the damping $\zeta_{\mathrm{CMS}}$ and the modal parameters of the transmissbility $T_{y_{\text {cor }}, a_{y}}(s)$ (Eq. 5.8). As shown in Table 1, the latter are dependent on the fluid density of the medium in the CMFM-tube. Therefore an active version of the CMS is suggested. In Fig. 5.6 the passive model (Fig. 5.3) is extended with an extra sensor $a_{1}$, to measure the accelerations of the mass $m_{1}$, and an actuator $F_{\mathrm{a}}$ to apply a force on the mass $m_{1}$.

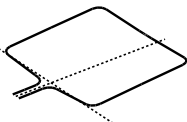




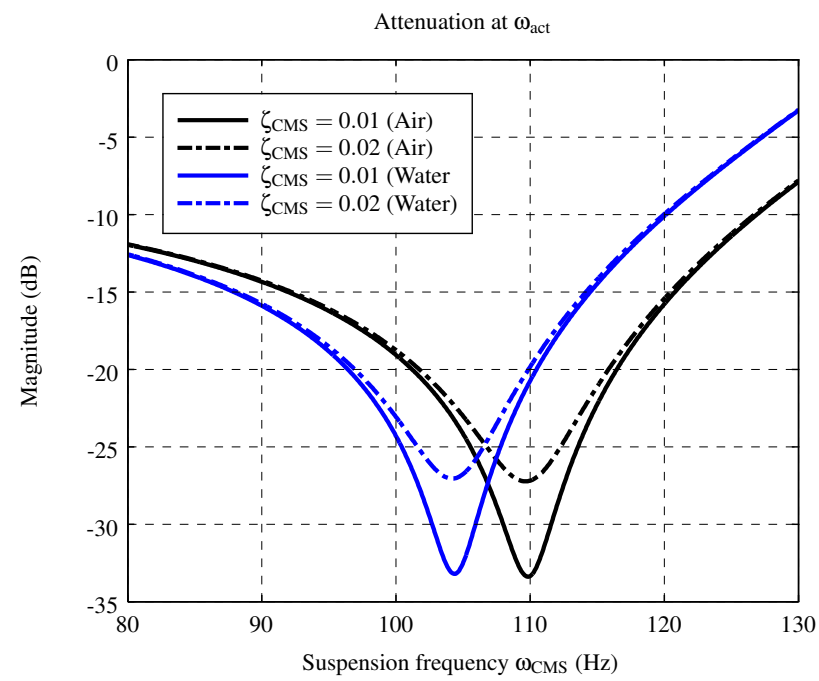

Fig. 5.5 Influence of the suspension frequency $\omega_{\text {CMS }}$ on the attenuation of at the actuation frequency $\omega_{\text {act }}$ for two different fluids (air and water) and the dampings ratio $\zeta_{\mathrm{CMS}}$ of the CMS.

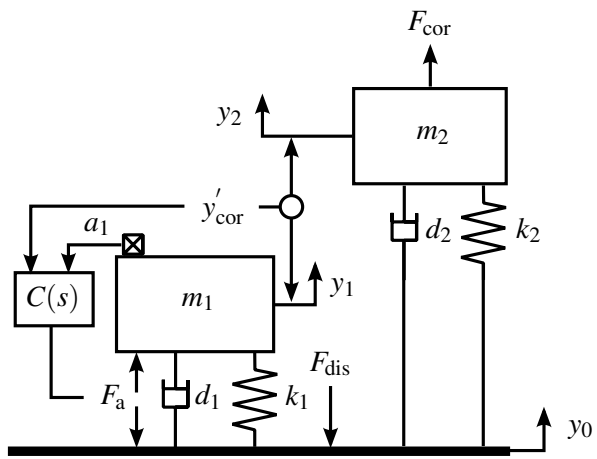

Fig. 5.6 1D CMFM active CMS model. Compared to the CMFM CMS model (Fig.5.3) actuation and sensing means are added to flexibly suspended mass $m_{1}$.

A transfer function between the input $F$ to the output $a_{1}$ is derived as:

$$
P(s)=\frac{a_{1}(s)}{F_{\mathrm{a}}(s)}=\frac{1}{m_{1}} \cdot \frac{s^{2}}{s^{2}+\frac{d_{1}}{m_{1}} s+\frac{k_{1}}{m_{1}}}
$$

Proportional acceleration feedback is proposed to add extra mass and thus decrease the initial suspension frequency, which is set at $\omega_{\mathrm{CMS}}=116 \cdot 2 \pi \mathrm{rad} / \mathrm{s}$.

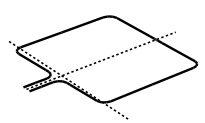




$$
C_{a_{1}}(s)=\frac{F_{\mathrm{a}}(s)}{a_{1}(s)}=-k_{\mathrm{a}}
$$

For this controller, the transmissibility from external vibrations to the displacement of the mass is equal to:

$$
T_{q_{1}, a_{y}}(s)=\frac{q_{1}(s)}{a_{y}(s)}=\frac{-1}{\left(m_{1}+k_{\mathrm{a}}\right) s^{2}+d_{1} s+k_{1}}
$$

resulting in a new suspension frequency $\omega_{\mathrm{CMS} \text {,active }}=\sqrt{\frac{k_{1}}{m_{1}+k_{\mathrm{a}}}}$, where the gain $k_{\mathrm{a}}$ of the feedback can be adjusted to achieve the desired suspension frequency:

$$
k_{\mathrm{a}}=m_{1}\left(\frac{\omega_{\mathrm{CMS}, \text { passive }}^{2}}{\omega_{\mathrm{CMS} \text {,active }}^{2}}-1\right)
$$

A second advantage of an active CMS, is the possibility to add relative damping to the measured, relative displacement $y_{\text {cor }}^{\prime}$. This limits the effect of shock disturbances on the amplitude of the undamped modes. The added damping prevents the relative displacement to go beyond the measurement range. To damp the internal mode, positive position feedback is added in combination with a notch filter at the actuation frequency [52]. This notch filter is added to prevent a change in the transfer function between $F_{\text {cor }}(s)$ and $y_{\text {cor }}^{\prime}(s)$ and thus in a change in sensitivity for a mass-flow. Therefore the controller is equal to:

$$
\begin{aligned}
C_{y_{\text {cor }}}(s) & =\frac{F_{\mathrm{a}}(s)}{y_{\text {cor }}(s)} \\
& =k_{\mathrm{p}} \cdot \underbrace{\frac{\omega_{\text {cor }}^{2}}{s^{2}+0.01 \omega_{\text {cor }} s+\omega_{\text {cor }}^{2}}}_{\text {LowPass }} \cdot \underbrace{\frac{s^{2}+0.0001 \omega_{\mathrm{act}} s+\omega_{\mathrm{act}}^{2}}{s^{2}+0.1 \omega_{\mathrm{act}} s+\omega_{\mathrm{act}}^{2}}}_{\text {Notch }}
\end{aligned}
$$

In Fig. 5.7 the new transmissibility is depicted for the active CMS. Showing that an attenuation of more than $20 \mathrm{~dB}$ is possible. In the magnified part of the figure is shown that the magnitude of the resonance peak is lowered due to the controller presented in Eq. 5.14.

\subsection{Mechanical design}

In the previous section a concept is proposed to reduce the influence of external vibrations on the measured sensor displacements. In this section the conceptual design is extended to a mechanical design.

First, the model of the reference system (Fig. 5.1) and the 1D active CMS (Fig. 5.6) are combined. The combined model is depicted in Fig. 5.8. The sensors measuring the tube displacement $\left(s_{1}\right.$ and $\left.s_{2}\right)$ are no longer measuring it relative to the casing, due to the introduction 


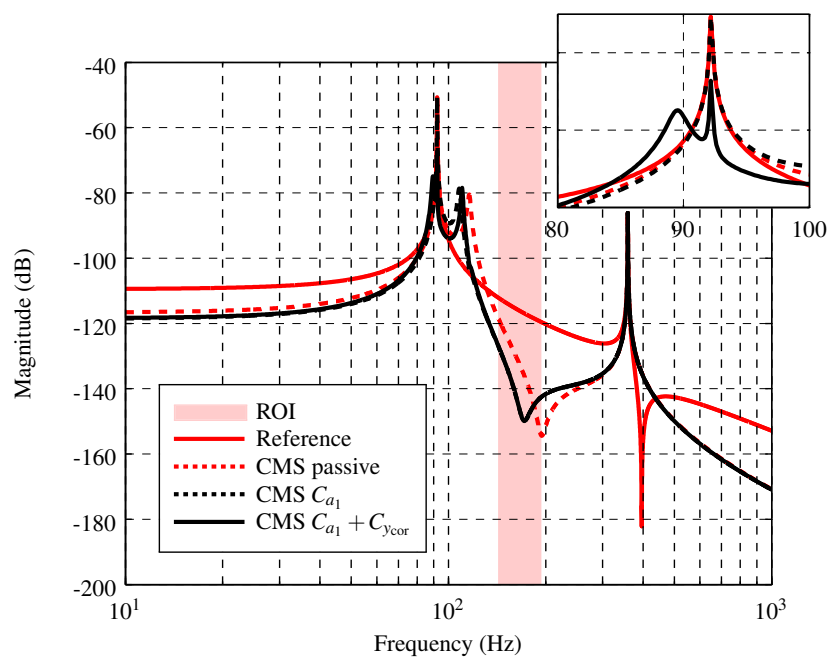

Fig. 5.7 Transmissibility from external vibrations $a_{y}$ to the Coriolis displacement $y_{\text {cor }}$ of the reference system (Eq.5.5) and the CMS system (Eq. 5.8. The region of interest is a $25 \mathrm{~Hz}$ band around the actuation frequency $\omega_{\text {act }}$. The CMS transmissibility is depicted for the passive system and for the active system, whereby the controllers of Eq. 5.11 and 5.14 are used.

of an active flexible element. This element represents a 1 Degree-Of-Freedom (DOF) compliant mechanism including an actuator in the compliant direction.

The eventual realisation of the CMS is presented in Fig. 5.10, The design comprehends five leaf-flexures, realising the stiffness $k_{1}$. The five flexures are configured to have an exact constraint design with one remaining DOF [57]. The optic displacement sensors, including the sensor mounting structure, together constitute mass $m_{1}$. A voice coil actuator is added to apply a force between the casing mounting structure and mass $m_{1}$. A threaded rod connects the displacement sensors structure, the five leaf-flexures and the coil of the actuator together. On this this rod an accelerometer is mounted to measure the accelerations of the compliant structure. A photo of the final result is depicted in Fig. 5.9

\subsection{Experimental validation}

In this section the design presented in the previous section is experimentally validated. In the first subsection the setup is explained. In the second subsection the modelled and experimentally obtained transmissibility functions are compared. Finally, in the third subsection the sensitivity of the mass-flow measurement to external vibrations RMS value is given for the new design.

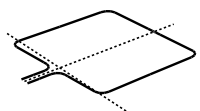




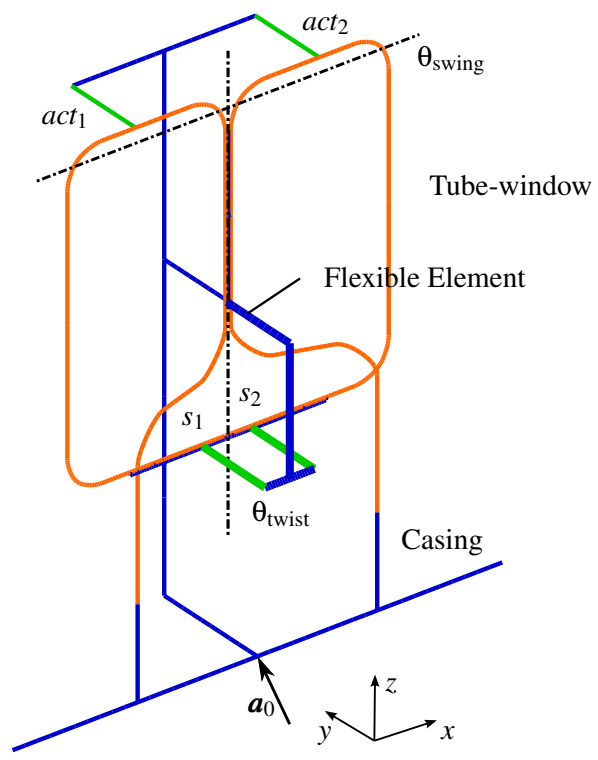

Fig. 5.8 Multibody model of the CMFM CMS. Compared to the CMFM model (Fig. 5.1) the tube displacements $\left(s_{1}\right.$ and $\left.s_{2}\right)$ are not measured relative to the casing any more.

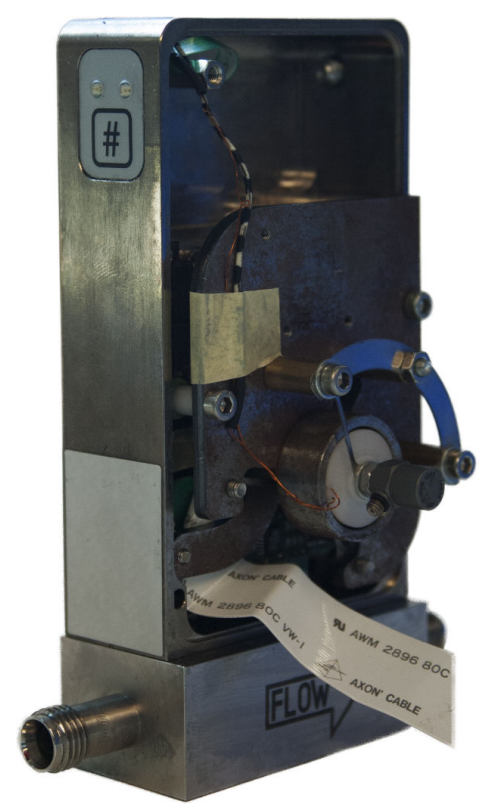

Fig. 5.9 Photo of CMFM including the active CMS module.

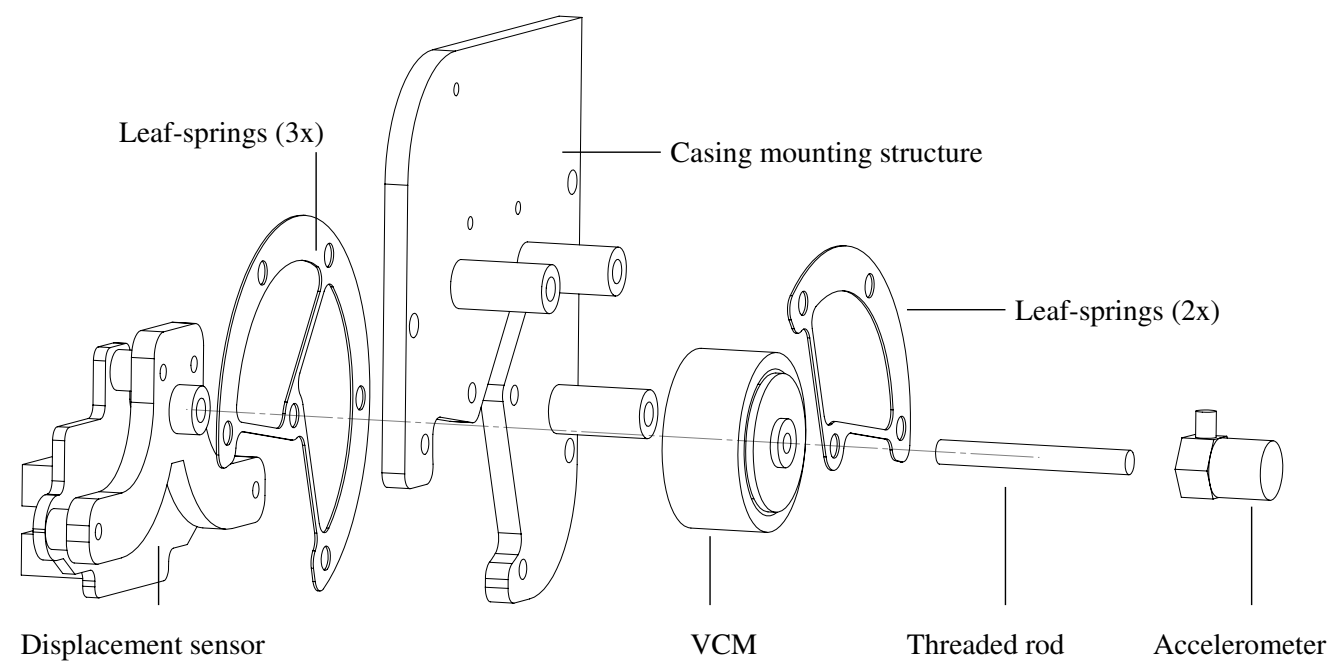

Fig. 5.10 Mechanical design of the 1-DOF active CMS. The 1-DOF is realised between the casing and the tube-displacement sensor and is sensed by an accelerometer en actuated by a VCM. 


\subsubsection{Setup}

The experimental setup is depicted in Fig. 5.11. The device under test (DUT) is a functional model of a CMFM including the CSM (Fig. 5.9). The CSM design is presented in section 5.4 and more details of the patented design of a CMFM are presented in [45]. The DUT is placed on a 6-DOF shaker to be able to apply external vibrations. A broadband white disturbance, a multi-sine between 5 and $500 \mathrm{~Hz}$, is applied on the 6-DOF platform, using the six actuators. More details of the shaker-setup are given in [64]. The active strategy, using a Brül\&Kjær 4393 accelerometer, is implemented using MATLAB XPC Target in combination with a NI6259 data acquisition card. The real-time system runs at a sample rate of $10 \mathrm{kHz}$.

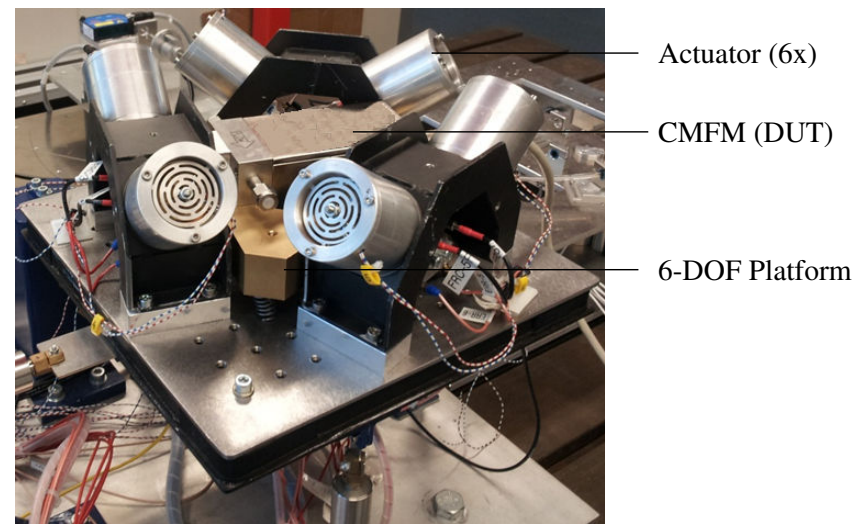

Fig. 5.11 6-DOF shaker platform, including Device Unter Test (DUT), to apply an external vibrations on the casing of a CMFM.

\subsubsection{Transmissibility}

First the transfer function from an actuator input $U$ in Volts to the acceleration sensor $a_{1}$ is validated, where $F_{\mathrm{a}}=\frac{k_{\mathrm{m}}}{R} U$ with a motor constant $k_{\mathrm{m}}$ and resistance $R$ of the voice coil actuator. From an experiment the equivalent mass is estimated: $m_{\mathrm{eq}}=\frac{m_{1} R}{k_{\mathrm{m}}}=4.8 \frac{\mathrm{Vs}}{\mathrm{m}}$. Besides the physical mass, this includes the characteristics of the actuator, the used amplifier and the accelerometer signal conditioner. The measured transfer function is compared with the model (Eq. 5.10) in Fig. 5.12 From this experiment the relevant CMS parameters can be estimated. The resonance frequency is $\omega_{\mathrm{CMS} \text {,passive }} \approx 116 \cdot 2 \pi \mathrm{rad} / \mathrm{s}$ and the damping $\zeta_{\mathrm{CMS}} \approx$ 0.01. Around $375 \mathrm{~Hz}$ an internal mode of the CMS is visible.

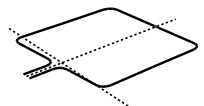




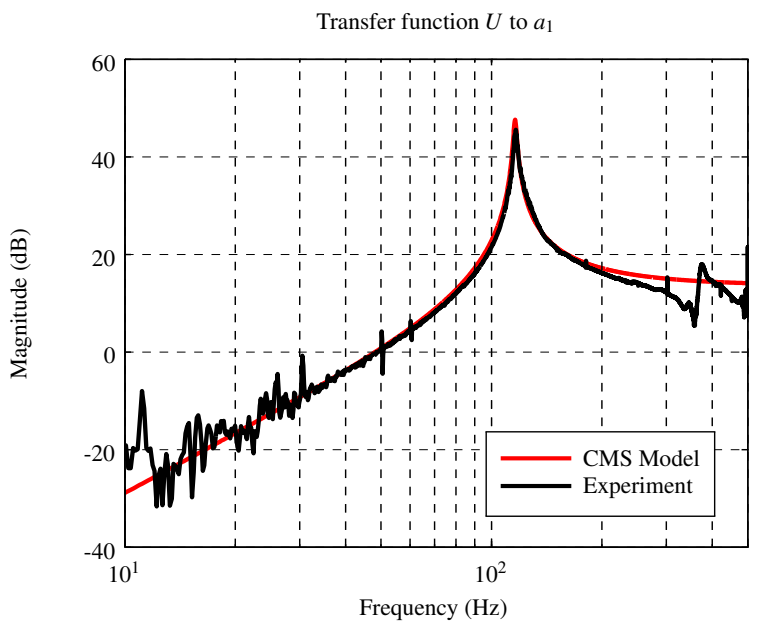

Fig. 5.12 Transfer function from $U$ to $a_{1}$. The experimental result is compared to the model (Eq. 5.10) whereby for the model $F_{\mathrm{a}}=\frac{m_{1}}{m_{\mathrm{eq}}} U$ and $\frac{k_{1}}{m_{1}}=\omega_{\mathrm{CMS} \text {,passive }}^{2}$ are used.

Secondly, the transmissibility $T_{y_{\text {cor }}^{\prime}, a_{y}}(s)$ is validated. The result is depicted in Fig. 5.13 when the fluid medium is air. The resonance frequencies of the tube are slightly lower than presented in Table 5.1. Further, the effect of lowering the resonance frequency $\omega_{\mathrm{CMS}}$ is shown, using the active strategy presented in section 5.3.3 Whereby, the CMS suspension frequency $\omega_{\mathrm{CMS} \text {,passive }}=116 \cdot 2 \pi \mathrm{rad} / \mathrm{s}$ actively is reduced to $\omega_{\mathrm{CMS} \text {,active }}=110 \cdot 2 \pi \mathrm{rad} / \mathrm{s}$. It is clearly visible that the anti-resonance is shifting as function of $\omega_{\mathrm{CMS}}$. Resulting in more than $20 \mathrm{~dB}$ attenuation in the region of interest.

Further, the resonance frequencies of the CMFM are determined for two different fluid densities and presented in Table 5.2. The values are slightly different than the model values, which are given in Table 5.1. This means that the CMS design parameters for a minimal influence of external vibrations, cannot be achieved from Fig. 5.5

Table 5.2 Estimated CMFM resonance frequencies for different fluid densities $\rho$.

\begin{tabular}{rccc}
\hline$\rho\left(\mathrm{kg} / \mathrm{m}^{3}\right)$ & $\omega_{\text {cor }, 1}(\mathrm{rad} / \mathrm{s})$ & $\omega_{\text {cor }, 2}(\mathrm{rad} / \mathrm{s})$ & $\omega_{\text {act }}(\mathrm{rad} / \mathrm{s})$ \\
\hline $1($ Air $)$ & $92.19 \cdot 2 \pi$ & $359.8 \cdot 2 \pi$ & $168.2 \cdot 2 \pi$ \\
$998($ Water $)$ & $87.70 \cdot 2 \pi$ & $337.8 \cdot 2 \pi$ & $158.1 \cdot 2 \pi$ \\
\hline
\end{tabular}




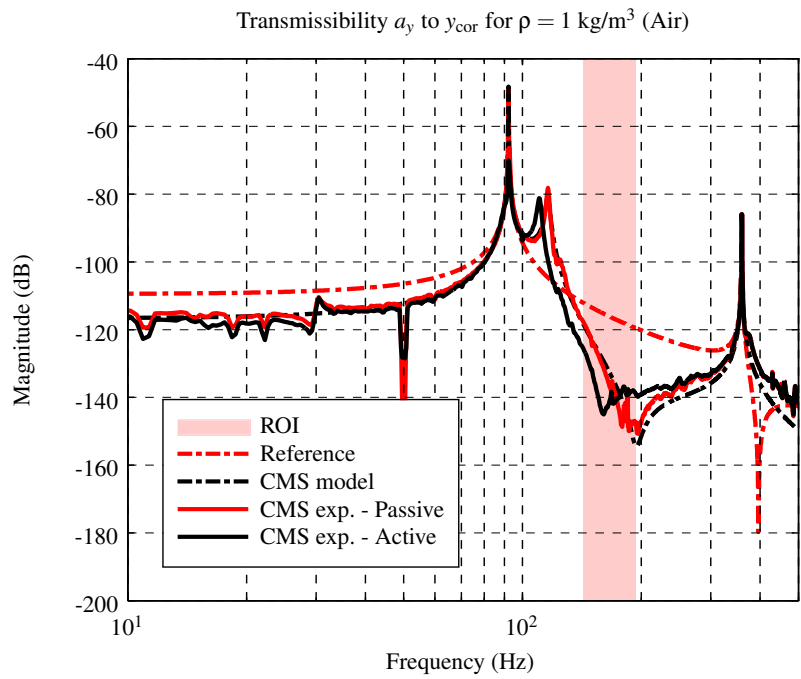

Fig. 5.13 Validation of the transmissibility from external vibrations $a_{y}$ to the Coriolis displacement $y_{\text {cor }}$ of the CMS system (Eq. 5.8) for the passive and active CMS compared to the model. The CMS suspension frequency $\omega_{\mathrm{CMS} \text {,passive }}=116 \cdot 2 \pi \mathrm{rad} / \mathrm{s}$ is actively reduced to $\omega_{\mathrm{CMS} \text {, active }}=110 \cdot 2 \pi \mathrm{rad} / \mathrm{s}$. Model parameters are based on the estimated parameters when the CMFM tube is filled with air.

\subsubsection{Disturbance sensitivity}

In the previous section the transmissibility function is validated. In earlier research it was shown that minimising this function in the region of interest results in a lower disturbance sensitivity (Chapter 2). In that paper the influence of broadband external vibrations on the RMS measurement error of a CMFM is explained and validated. The influence is dominated by the gain of the transmissibility at the actuation frequency, the sensitivity for the mass-flow and the width of the bandpass filter in the mass-flow measurement algorithm. In this subsection the influence of external vibrations on the mass-flow measurement value, in combination with a $10 \mathrm{~Hz}$ bandpass filter, is shown for the newly presented design.

Table 5.3 RMS measurement error (in normalised units), including attenuation in dB compared to the reference, for the different applied disturbance levels. The values are depicted in Fig. 5.14

\begin{tabular}{rrrrrrrrrr}
\hline $\begin{array}{r}\text { Disturbance } \\
\left(\left(\mathrm{m} / \mathrm{s}^{2}\right)^{2} / \mathrm{Hz}\right)\end{array}$ & $\begin{array}{r}\text { Reference } \\
\text { (units) }\end{array}$ & \multicolumn{2}{c}{ Passive (air) } & \multicolumn{3}{c}{ Active (Air) } & \multicolumn{2}{c}{ Passive (water) } & \multicolumn{2}{c}{ Active (water) } \\
\hline 0 & 1.000 & 0.628 & -4.05 & 0.693 & -3.19 & 0.733 & -2.70 & 0.974 & -0.23 \\
$1 \mathrm{e}-7$ & 1.457 & 0.666 & -6.80 & 0.686 & -6.54 & 0.784 & -5.39 & 0.997 & -3.30 \\
$1 \mathrm{e}-6$ & 4.577 & 0.700 & -16.3 & 0.765 & -15.5 & 1.436 & -10.1 & 1.031 & -12.9 \\
$1 \mathrm{e}-5$ & 13.87 & 1.296 & -20.6 & 0.998 & -22.9 & 3.692 & -11.5 & 1.046 & -22.5 \\
$1 \mathrm{e}-4$ & 45.33 & 4.754 & -19.6 & 2.764 & -24.3 & 12.56 & -11.2 & 4.137 & -20.8 \\
$4 \mathrm{e}-4$ & 93.36 & 13.80 & -16.6 & 9.325 & -20.0 & 26.44 & -11.0 & 8.411 & -20.9 \\
\hline
\end{tabular}

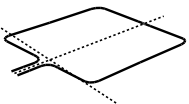




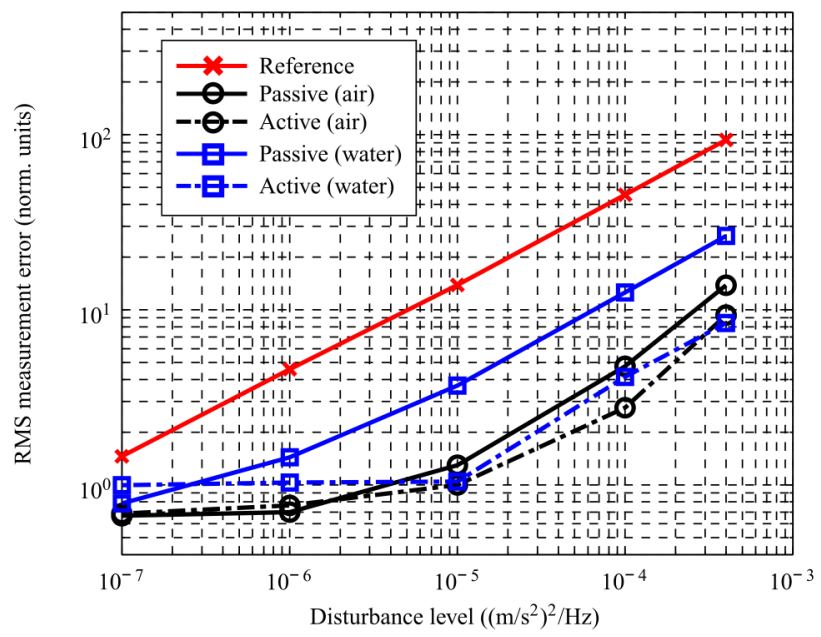

Fig. 5.14 RMS measurement error for the different applied disturbance levels. Numerical values are given in Table 5.3

Therefor a broadband multi-sine disturbance between 5 and $500 \mathrm{~Hz}$ is applied on the casing of the CMFM. In Table 5.3 the normalised RMS measurement error is shown for different disturbance levels and the different configurations. The data is also graphically shown in Fig. 5.14 The RMS errors are compared to the reference instrument (without CMS) resulting in an attenuation in $\mathrm{dB}$. The influence is determined for both cases when the tube is filled with air and water. For the active configurations the resonance frequency of the CMS is actively lowered, using the strategy as presented in section 5.3.3. For the active CMS, the suspension frequency $\omega_{\mathrm{CMS}}$ is lowered to respectively $113 \cdot 2 \pi \mathrm{rad} / \mathrm{s}$ and $109 \cdot 2 \pi \mathrm{rad} / \mathrm{s}$ for the the case the fluid medium is air or water. Those values are different than the values depicted in Fig. 5.5 because the modal parameters of the CMFM are different for the CMFM compared to the model (See Table 5.1 and 5.2).

From the results is concluded that the parameters of the passive CMS are not optimal for the both cases when the tube is filled with air or with water. Actively tuning the resonance frequency improves the result. For low external disturbance levels the attenuation is small, because of the noise floor of the tube displacement sensors. This is also visible in Fig. 5.14. for low disturbance levels the error line trend is horizontal due to the noise level of the tube displacement sensors.

The optimal CMS suspension parameters are obtained when the transmissibility $T_{y_{\text {cor }}, a_{0}}(s)$ is minimal around the actuation frequency. In Appendix 5.8 the an approximation of the optimal CMS suspension frequency $\omega_{\mathrm{CMS}}$ is determined as function of the modal parameters $g_{1}, g_{2}, \omega_{\text {cor }, 1}, \omega_{\text {cor }, 2}$ and $\omega_{\text {act }}$. Experiments shows that an attenuation of more than $24 \mathrm{~dB}$ can be achieved by the use of an active CMS.

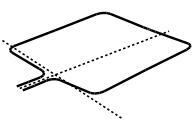




\subsection{Discussion}

In the previous section the achievable attenuation of the vibration sensitivity of a CMFM by a CMS is presented. The attenuation of a passive CMS is dependent on both the design of the fluid-conveying tube and the compliantly mounted sensor module. The dynamic properties of the tube alter for different fluid densities. Therefore the performance is dependent on the density of the fluid.

The sensitivity for the properties of the tube can be reduced by using an active CMS to lower the suspension frequency. For robustly stable control the suspension frequency can only be lowered. A disadvantage is that the feedback introduces extra damping, due to back-emf, resulting in less attenuation, but this can be resolved using the actuator in current-mode. In future, the optimal parameters can be found when using adaptive feedback control. Alternatively to the active feedback method, a compromise can be found, when the design parameters of the CMS for high and low fluid densities are averaged. For example, the CMS can be designed with an suspension frequency $\omega_{\mathrm{CMS}}=107 \cdot 2 \pi \mathrm{rad} / \mathrm{s}$, to achieve a combined maximum attenuation (See Fig. 5.5).

The presented CMS module is a proof of principle and is rather large, but the concept can be miniaturised, for example like the optic reading head in DVD-players. Further, instead of an acceleration sensor also displacement or velocity sensors can be used to alter the dynamic behaviour of the CMS. Another advantage of the CMS is that the influence of the gravitational sagging is minimal. Therefore, the displacement sensors are always in the same part of the characteristic sensor response curve, independent of the orientation of the instrument.

The presented CMS module can also be used in combination with other vibration reduction techniques, e.g. passive suspension between the casing and the surroundings (Chapter 2) or extended with active vibration isolation control (Chapter 3), to increase the reduction even more.

\subsection{Conclusions}

Vibration isolation for a Coriolis Mass-Flow Meter (CMFM) is achieved using an actively compliantly mounted sensor (CMS). More than $24 \mathrm{~dB}$ attenuation is realised compared to the reference system. The CMFM measurement value depends on a displacement measurement of the fluid-conveying tube movements. The measurement signal is also influenced by external vibrations. To achieve the $24 \mathrm{~dB}$ attenuation, a new design is introduced: A compliance between the casing and the displacement sensor is added, to enable a true displacement measurement of the tube.

Optimal attenuation is achieved by tuning the resonance frequency and the relative damping of the CMS. The optimal values depend on the dynamic properties of the tube, which change by the density of the fluid inside the tube. The parameters of the CMS are tuned actively, with acceleration feedback, to have a maximum attenuation, independently of the fluid density. An attenuation of more than $24 \mathrm{~dB}$ is demonstrated for a CMFM with an active CMS, in case the tube filled with both water and air. 


\subsection{Appendix: Optimal CMS frequency}

The achievable attenuation of the influence of external vibrations is dependent on the location of the zero of Eq. 5.8, as depicted in Fig. 5.4. This zero location is dependent on several modal parameters $\left(g_{1}, g_{2}, \omega_{\mathrm{cor}, 1}, \omega_{\mathrm{cor}, 2}, \omega_{\mathrm{act}}, Q, \omega_{\mathrm{CMS}}\right.$ and $\left.\zeta_{\mathrm{CMS}}\right)$. From Eq. 5.8 the location of the zero can be estimated by solving:

$$
\begin{aligned}
& g_{1}\left(s^{2}+\frac{1}{Q} \omega_{\mathrm{cor}, 2} s+\omega_{\mathrm{cor}, 2}^{2}\right)\left(s^{2}+2 \zeta_{\mathrm{CMS}} \omega_{\mathrm{CMS}} s+\omega_{\mathrm{CMS}}^{2}\right) \\
& +g_{2}\left(s^{2}+\frac{1}{Q} \omega_{\mathrm{cor}, 1} s+\omega_{\mathrm{cor}, 1}^{2}\right)\left(s^{2}+2 \zeta_{\mathrm{CMS}} \omega_{\mathrm{CMS}} s+\omega_{\mathrm{CMS}}^{2}\right) \\
& +\left(s^{2}+\frac{1}{Q} \omega_{\mathrm{cor}, 1} s+\omega_{\mathrm{cor}, 1}^{2}\right)\left(s^{2}+\frac{1}{Q} \omega_{\mathrm{cor}, 2} s+\omega_{\mathrm{cor}, 2}^{2}\right)=0
\end{aligned}
$$

To minimise the attenuation at the actuation frequency, the zero should be placed at this frequency, so $s=\mathrm{i} \omega_{\text {act }}$. Now Eq. 5.15 can be solved for $\omega_{\mathrm{CMS}}$ :

$$
\begin{aligned}
& \omega_{\mathrm{CMS}} \approx \\
& \sqrt{-\left(\left(g_{1}+g_{2}\right) \omega_{\mathrm{act}}^{2}-g_{1} \omega_{\mathrm{cor}, 2}^{2}-g_{2} \omega_{\mathrm{cor}, 1}^{2}\right) \cdot \ldots} \\
& \overline{\left(\omega_{\mathrm{act}}^{2} \omega_{\mathrm{cor}, 1}^{2}+\omega_{\mathrm{act}}^{2} \omega_{\mathrm{cor}, 2}^{2}-\omega_{\mathrm{cor}, 1}^{2} \omega_{\mathrm{cor}, 2}^{2}-\ldots\right.} \\
& \overline{\left.\left(g_{1}+g_{2}+1\right) \omega_{\mathrm{act}}^{4}+g_{1} \omega_{\mathrm{act}}^{2} \omega_{\mathrm{cor}, 2}^{2}+g_{2} \omega_{\mathrm{act}}^{2} \omega_{\mathrm{cor}, 1}^{2}\right)} \\
& /\left(\left(g_{1}+g_{2}\right) \omega_{\mathrm{act}}^{2}-g_{1} \omega_{\mathrm{cor}, 2}^{2}-g_{2} \omega_{\mathrm{cor}, 1}^{2}\right)
\end{aligned}
$$

In this approximation the damping parameters are omitted $\left(Q=\infty\right.$ and $\left.\zeta_{\mathrm{CMS}}=0\right)$, because those do not change the location of the minimum (see Fig. 5.5), they only affects the achievable attenuation. With this equation, the optimal CMS frequency can be calculated, when the needed modal parameters are estimated. 


\section{Chapter 6 \\ Evaluation}

The design rules for the next generation CMFMs for low flows $(<1 \mathrm{~kg} / \mathrm{h})$ are evaluated in this chapter. First the main performance criteria of a CMFM, based on a simple model, are discussed. Secondly, different CMFM tube designs are evaluated on their performance. In the third section the configurations analysed in this thesis are shown and it is discussed how those configurations can be used in commercial products.

\subsection{Performance criteria}

A CMFM is an active device based on the Coriolis force principle for direct mass-flow measurements independently of fluid properties [1, 78]. In this section a simple modal model of a CMFM is presented. Such model can be used to represent complex tube shapes as well, as validated in Chapter 2. Based on the model parameters, the flow range and the measurement sensitivity for flow are determined. Next, the sensitivity for disturbances is determined, with the focus on external vibrations.

\subsubsection{Modelling}

In the past, many examples have been presented that show how the fluid-tube interaction, including the Coriolis effect, can be modelled [25, 35, 54, 61, 72]. The modelling shows a CMFM sensitive for a mass-flow, but also that the measurement value is affected by all kind of disturbances. A summary of those disturbances is given in section 1.2 . In Appendix $\mathrm{B}$ a model of a simple CMFM with two degrees-of-freedom is derived. These two degrees-offreedom represent the Coriolis and actuation mode only. The model is depicted in Fig. 6.1. 


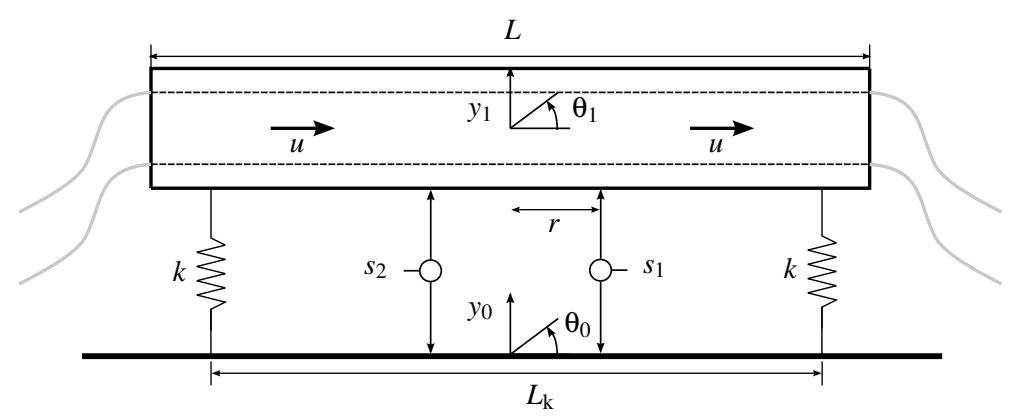

Fig. 6.1 2-DOF CMFM model: a rigid fluid-conveying tube of length $L$, flexibly mounted to the surroundings by stiffness $k$. The tube-movements are measured by two sensors, placed on equal distance $r$ of the rotation axis.

Based on this model, the equations of motion, for a symmetric undamped system with a constant mass-flow $\dot{\Phi}_{\mathrm{m}}=\rho_{\mathrm{f}} A_{\mathrm{f}} u$, are derived:

$$
\left[\begin{array}{cc}
m+\rho_{\mathrm{f}} A_{\mathrm{f}} L & 0 \\
0 & J+\frac{1}{12} \rho_{\mathrm{f}} A_{\mathrm{f}} L^{3}
\end{array}\right]\left[\begin{array}{l}
\ddot{y}_{1} \\
\ddot{\theta}_{1}
\end{array}\right]+\left[\begin{array}{cc}
0 & \dot{\Phi}_{\mathrm{m}} L \\
-\dot{\Phi}_{\mathrm{m}} L & 0
\end{array}\right]\left[\begin{array}{c}
\dot{y}_{1} \\
\dot{\theta}_{1}
\end{array}\right]+\left[\begin{array}{cc}
2 k & 0 \\
0 & \frac{1}{2} k L_{\mathrm{k}}^{2}
\end{array}\right]\left[\begin{array}{l}
y_{1} \\
\theta_{1}
\end{array}\right]=\left[\begin{array}{c}
F_{y}+2 k y_{0} \\
M_{\theta}+\frac{1}{2} k L_{\mathrm{k}}^{2} \theta_{0}
\end{array}\right]
$$

Where $m$ and $J=\frac{1}{12} m L^{2}$ are respectively the modal mass and modal moment of inertia of an empty tube. The tube has a length $L$ and a modal stiffness $k$ and $L_{\mathrm{k}}$ is the ratio between the translational and rotational stiffness. The cross sectional area of the fluid is $A_{\mathrm{f}}=\frac{1}{4} \pi d^{2}$, where the fluid has a density $\rho_{\mathrm{f}}$ and flow velocity $u$. The structural damping is omitted in this model, because the Coriolis tubes are normally designed with high Q-factors [44]. Using a modal decomposition method even CMFM models with complex tube shapes can be reduced to this simple model with only the Coriolis mode and actuation mode. This approximation is validated in Chapter 2 .

For the no-flow condition $(u=0)$, the tube has two clear resonance frequencies:

$$
\omega_{1}=\sqrt{\frac{2 k}{m+\rho_{\mathrm{f}} A_{\mathrm{f}} L}}, \quad \omega_{2}=\sqrt{\frac{\frac{1}{2} k L_{\mathrm{k}}^{2}}{J+\frac{1}{12} \rho_{\mathrm{f}} A_{\mathrm{f}} L^{3}}}=\frac{\sqrt{3} L_{\mathrm{k}}}{L} \sqrt{\frac{2 k}{m+\rho_{\mathrm{f}} A_{\mathrm{f}} L}}=\alpha \omega_{1},
$$

belonging respectively to the translational and rotational mode of the tube. The resonance frequencies depend on the geometry, but also on the density $\rho_{\mathrm{f}}$ of the fluid. The model is used to identify the effects of scaling down the flow-range of the CMFM [26]. The main dimensions of the CMFM are the length $L$ and the diameter $d$ of the tube. The mass scales with the length and diameter of the tube as $m \sim d^{2} L$. The modal stiffness $k$ in the model is due to the bending and torsion of the tube and also scales with the length and diameter as $k \sim \frac{d^{4}}{L^{3}}$. Thus the resonance frequencies scales with $\omega \sim \frac{d}{L^{2}}$.

The fluid conveying tube is actuated to oscillate in resonance with a low amplitude. A fluid flow in the vibrating tube induces Coriolis forces, proportional to the mass-flow, which 
affect the modeshape of the actuation mode, which is modelled by the coupling terms in the damping matrix (see Eq.6.1). To measure the change of the modeshapes, two relative-motion sensors are added to the model, located at equal distance $r$ from the rotation axis of actuation:

$$
\left[\begin{array}{l}
s_{1} \\
s_{2}
\end{array}\right]=\left[\begin{array}{cccc}
-1 & 1 & -r & r \\
-1 & 1 & r & -r
\end{array}\right]\left[\begin{array}{l}
y_{0} \\
y_{1} \\
\theta_{0} \\
\theta_{1}
\end{array}\right]
$$

Using these two sensor signals, the modeshape can be measured and the mass-flow $\dot{\Phi}_{\mathrm{m}}$ can be calculated.

In the remainder of this subsection, the measurement sensitivity for mass-flow of the model, depicted in Fig. 6.1 is determined. The mass-flow measurement sensitivity is calculated for two cases, a translational and a rotational actuation mode.

The tube is actuated to resonate in one of its modeshapes. The Coriolis-effect results in a 90 degree out-of-phase actuation of the other modeshape. The actuation displacement and the induced Coriolis displacement both occur at the actuation frequency. Two sensors, on both sides of the rotation axis, measure the translation and rotation of the tube, ideally just one harmonic. The phase difference between both sensors, in case the translational mode is used as actuation mode, is approximated as (see Appendix [B]:

$$
\Delta \phi_{1} \approx 2 \frac{\mathfrak{I}\left(s_{1}-s_{2}\right)}{\mathfrak{R}\left(s_{1}+s_{2}\right)}=\frac{2 r L}{\left(J+\frac{1}{12} \rho_{\mathrm{f}} A_{\mathrm{f}} L^{3}\right)} \frac{\omega_{1}}{\omega_{2}^{2}-\omega_{1}^{2}} \dot{\Phi}_{\mathrm{m}}
$$

whereby the Fourier transforms of the sensor signals ( $s_{1}$ and $s_{2}$, see Eq. 6.3) at the translational resonance frequency $\omega_{1}$ are used, which are determined by evaluating the Laplace transform of the model (Eq. 6.1) for the argument $s=\mathrm{i} \omega_{1}$. In this phase difference calculation, the external displacements $\left(y_{0}=\theta_{0}=0\right)$ and external forces $\left(F_{y}=M_{\theta}=0\right)$ are omitted.

In practice the phase difference is estimated using a phase demodulation algorithm, this is explained in section 2.3.2. The phase difference is actuation frequency dependent, in contrast to the time difference:

$$
\Delta t_{1}=\frac{\Delta \phi_{1}}{\omega_{1}}=\frac{2 r L}{\left(J+\frac{1}{12} \rho_{\mathrm{f}} A_{\mathrm{f}} L^{3}\right)} \frac{1}{\omega_{2}^{2}-\omega_{1}^{2}} \dot{\Phi}_{\mathrm{m}}=\frac{24 r}{L} \frac{1}{2 k\left(\alpha^{2}-1\right)} \dot{\Phi}_{\mathrm{m}}
$$

The same holds, when the rotational mode is used as actuation mode:

$$
\begin{aligned}
& \Delta \phi_{2} \approx 2 \frac{\mathfrak{I}\left(s_{1}+s_{2}\right)}{\mathfrak{R}\left(s_{1}-s_{2}\right)}=\frac{2 L}{r\left(m+\rho_{\mathrm{f}} A_{\mathrm{f}} L\right)} \frac{\omega_{2}}{\omega_{2}^{2}-\omega_{1}^{2}} \dot{\Phi}_{\mathrm{m}} \\
& \Delta t_{2}=\frac{2 L}{r\left(m+\rho_{\mathrm{f}} A_{\mathrm{f}} L\right)} \frac{1}{\omega_{2}^{2}-\omega_{1}^{2}} \dot{\Phi}_{\mathrm{m}}=\frac{2 L}{r} \frac{1}{2 k\left(\alpha^{2}-1\right)} \dot{\Phi}_{\mathrm{m}}
\end{aligned}
$$


The measurement sensitivity for flow is defined as:

$$
S_{i}=\frac{\Delta t_{i}}{\dot{\Phi}_{\mathrm{m}}}
$$

The sensitivity is dependent on the sensor position ratio $\frac{r}{L}$, the tube stiffness $k$ and the frequency separation factor $\alpha$. Note that the sensitivity for flow is not a constant, e.g. the stiffness depends on the temperature.

\subsubsection{Pressure drop}

In the previous section a simple model of a CMFM is derived. No dimensions are given yet, because those dimensions are inherent to the flow range in which the sensor is used. The nominal flow through a CMFM is defined as the water flow at nominal temperature and pressure, resulting in 1 bar pressure drop over the instrument. The pressure drop across the tube can be estimated by the Darcy-Weisbach equation:

$$
\Delta P=\frac{f L \rho u^{2}}{2 d}
$$

besides the dependency on the length $L$ and diameter $d$ of the tube, the velocity $u$ and density $\rho_{\mathrm{f}}$ of the fluid, the pressure drop $\Delta P$ is dependent on the friction coefficient $f$, between the tube and the fluid. For a hydraulically smooth tube, this factor is defined as:

$$
\begin{array}{ll}
f_{\text {laminar }}=\frac{64}{R_{\mathrm{e}}} & \left(R_{\mathrm{e}}<2300\right) \\
f_{\text {turbulent }}=\frac{0.3164}{\sqrt[4]{R_{\mathrm{e}}}} & \left(R_{\mathrm{e}}>2300\right)
\end{array}
$$

For a circular tube, the Reynolds number is equal to:

$$
R_{\mathrm{e}}=\mathrm{v} u d
$$

where $v$ is the kinematic viscosity of the fluid. For $R_{\mathrm{e}}>2300$ the fluid is assumed to be turbulent. Using Eq. 6.96.12 the mass-flow $\dot{\Phi}_{\mathrm{m}}=\rho_{\mathrm{f}} A_{\mathrm{f}} u$ as function of the pressure drop is determined:

$$
\begin{array}{ll}
\dot{\Phi}_{\mathrm{m}}=\frac{\pi d^{4} \Delta P}{128 v L} & \left(R_{\mathrm{e}}<2300\right) \\
\dot{\Phi}_{\mathrm{m}}=\frac{\pi \rho^{\frac{3}{7}} d^{\frac{19}{7}} \Delta P^{\frac{4}{7}}}{1.3946 v^{\frac{1}{7}} L^{\frac{4}{7}}} & \left(R_{\mathrm{e}}>2300\right)
\end{array}
$$

In Fig. 6.2 the nominal flow, for 1 bar pressure drop and water as fluid (density $\rho_{\mathrm{f}}=$ $998 \mathrm{~kg} / \mathrm{m}^{3}$ and dynamic viscosity $\mathrm{v}=1 \mathrm{e} 6 \mathrm{~m}^{2} / \mathrm{s}$ ), is depicted with the assumption that the 


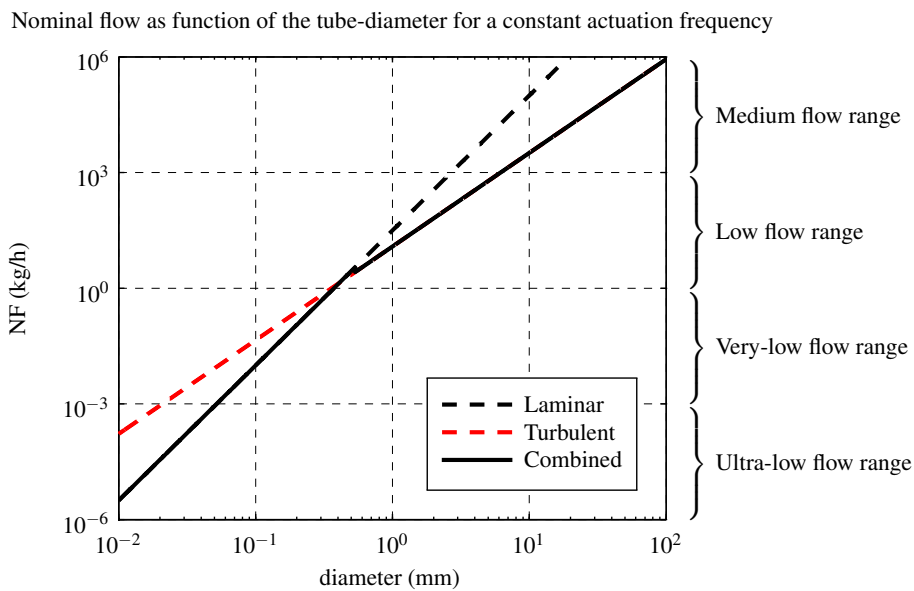

Fig. 6.2 The Nominal Flow (water flow at nominal temperature and pressure, resulting in 1 bar pressure drop) versus the tube diameter. The NF is different for laminar and turbulent flow. The flow ranges are adopted from Wang and Baker [78].

resonance frequencies $\left(\omega_{1}\right.$ and $\left.\omega_{2}\right)$ are constant, by scaling the length of the tube with its diameter $\left(L=L_{0}\left(\frac{d}{d_{0}}\right)^{\frac{1}{2}}=8.9 d^{\frac{1}{2}}\right)$. The nominal dimensions $L_{0}$ and $d_{0}$ of a functional model of a CMFM are used [45]. For smaller diameters the fluid becomes laminar and the pressure drop larger, resulting in a lower nominal flow. For a turbulent flow the nominal flow scales as $\dot{\Phi}_{\mathrm{m}} \sim d^{17 / 7}=d^{2.429}$. A similar correlation $\dot{\Phi}_{\mathrm{m}} \sim d^{2.312}$ for the turbulent flow regime was also observed by Wang and Baker [78] for different flow sensor designs of three manufactures.

\subsubsection{Sensitivity scaling}

In subsection 6.1.1 the measurement sensitivity for mass-flow of the model, depicted in Fig. 6.1, is determined. In this subsection the effect on the measurement sensitivity of scaling down the nominal flow range of a CMFM is analysed.

Reconsider Eq. 6.5 and 6.7, where the mass-flow measurement sensitivity is calculated for two cases, a translational and a rotational actuation mode. The sensitivity depends on the sensor position ratio $\frac{r}{L}$, the tube stiffness $k$ and the frequency separation factor $\alpha$. For a constant sensor position, actuation frequency and $\alpha$, the sensitivity scales with $S \sim d^{-\frac{5}{2}}$. The sensitivity as function of the nominal flow is depicted in Fig. 6.3. From this figure can be concluded, that when scaling down the instrument, the sensitivity for flow is decreasing. i.e. the sensitivity is lower than it would be, if the flow would stay turbulent. Secondly, the total measured phase or time difference is scaling down for decreasing laminar flows, while for turbulent flows this value is constant. 


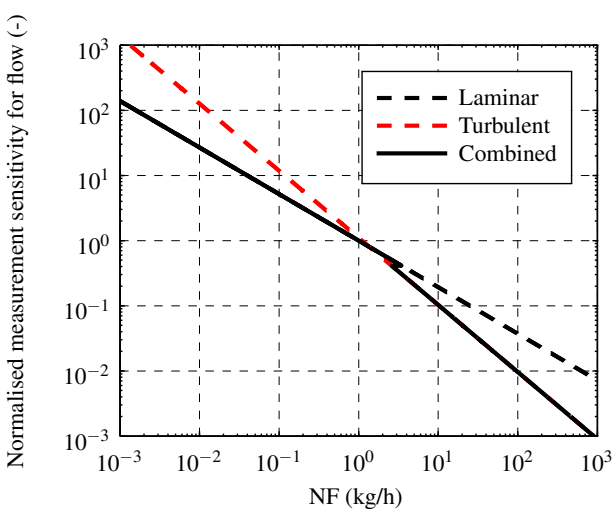

Fig. 6.3 The sensitivity for flow as function of the nominal flow. The scaling is different for laminar and turbulent flows. Result is normalised on the sensitivity belonging to $1 \mathrm{~kg} / \mathrm{h}$.

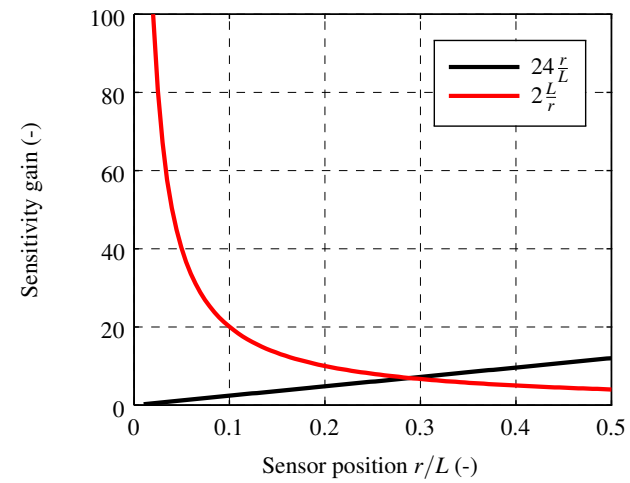

Fig. 6.4 The sensitivity gain of Eq. 6.5 and 6.7 as function of the sensor position, see Fig.6.1

In Fig. 6.4 the sensitivity gain, the sensitvity without the common factor $\frac{1}{2 k\left(\alpha^{2}-1\right)}$, as function of the sensor position $\frac{r}{L}$ is depicted. Clearly, the sensitivity can be boosted by actuating in rotation and placing the sensors close to the rotation axis. This way, the sensitivity deterioration for instruments with a lower nominal flow, in the laminar flow range, can be compensated for. The advantage of placing the sensors close to the rotation axis, is that sensors with a low measurement range can be used and therefore a high resolution.

In almost all commercial available CMFMs the translational mode is used as actuation mode. The rotational mode as actuation mode is used in Haneveld et al. [27], Mehendale [44], both with a very-low nominal flow (see Fig. 6.2) of respectively $1.2 \mathrm{~g} / \mathrm{h}$ and $100 \mathrm{~g} / \mathrm{h}$.

\subsubsection{Sensitivity for disturbances}

Besides measuring the mass-flow, a CMFM measurement is also sensitive to other effects. Several disturbances are already mentioned in section 1.2. In this subsection the influence of disturbances, mainly sensor noise and external vibrations, is discussed. Leading to a simple expression of the influence of external vibrations on the noise level of the CMFM measurement. This expression shows that influence of external vibrations is increasing when scaling down the nominal flow range of a CMFM.

The mass-flow is calculated from the measured phase difference and the measurement sensitivity (section 2.3.2). An estimation of the true mass-flow is given by:

$$
\dot{\Phi}_{\mathrm{m}, \mathrm{est}}=\frac{\Delta t_{\text {measure }}}{S}=\frac{\Delta \phi_{\text {measure }}}{\omega_{\text {act }} S}
$$

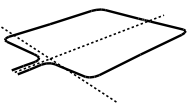




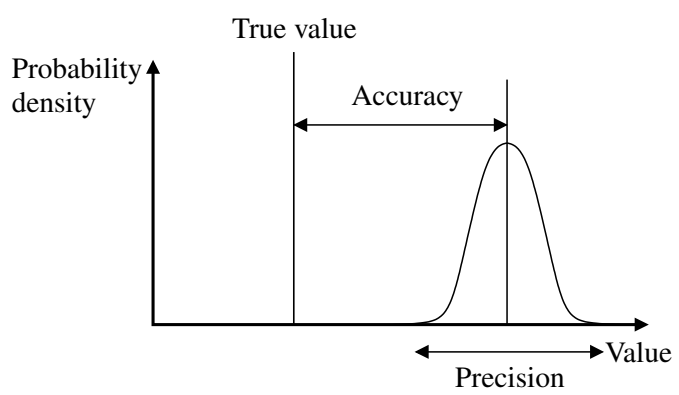

Fig. 6.5 Difference between the accuracy and the precision of the flow measurement value.

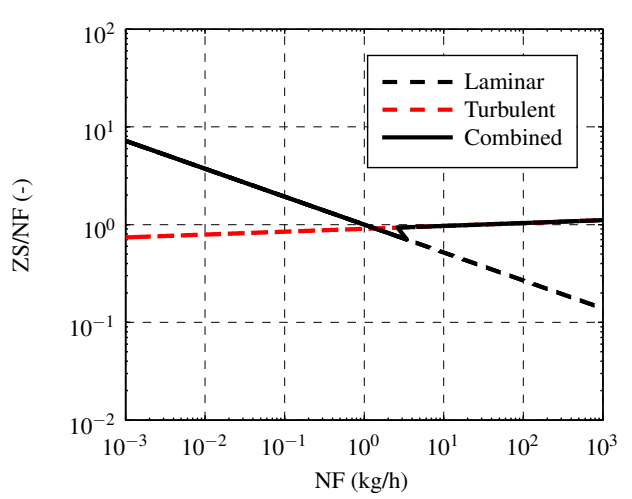

Fig. 6.6 The zero stability (ZS) as function of the nominal flow. Result is normalised on the ZS belonging to $1 \mathrm{~kg} / \mathrm{h}$.

Disturbances can influence the measurement of the phase difference and actuation frequency or change the calibrated sensitivity for flow. An offset in the phase difference or a change in the flow sensitivity can result in a drift of the measurement value, losing accuracy. A fluctuating phase difference results in a noisy measurement value, losing precision (see Fig. 6.5). The lower limit of the measurement range of a CMFM is specified by the zero stability. This value is dependent on the maximal measured phase difference, unrelated to a mass-flow, the measurement sensitivity and actuation frequency (Eq. 6.15):

$$
\mathrm{ZS}=\frac{\Delta \phi_{\text {noise }}}{\omega_{\text {act }} S}
$$

The zero stability value is normally specified by a percentage of the nominal flow. In Fig. 6.6 the zero stability value is depicted as function of the nominal flow for a constant actuation frequency and phase noise level. Thus, the zero stability scales with $\mathrm{ZS} \sim d^{\frac{5}{2}}$. For lower flow ranges the sensitivity for flow decreases, and thus the zero stability increases, decreasing the range-ability of a CMFM.

The measured phase difference is the sum of various contributions that are approximately independent. Those are, among others, a contribution due to a mass-flow, sensor noise and external vibrations. We focus on the effects of sensor noise and external vibrations, which are mainly affecting the precision of the the measurement value.

The phase difference can be approximated as the ratio of the actuation amplitude $y_{\text {act }}=$ $\frac{1}{2}\left(s_{1}-s_{2}\right)$ and Coriolis displacement amplitude $y_{\text {cor }}=\frac{1}{2}\left(s_{1}+s_{2}\right)($ Eq. 3.2):

$$
\Delta \phi \approx 2 \frac{\left|y_{\mathrm{cor}}\right|}{\left|y_{\mathrm{act}}\right|}
$$




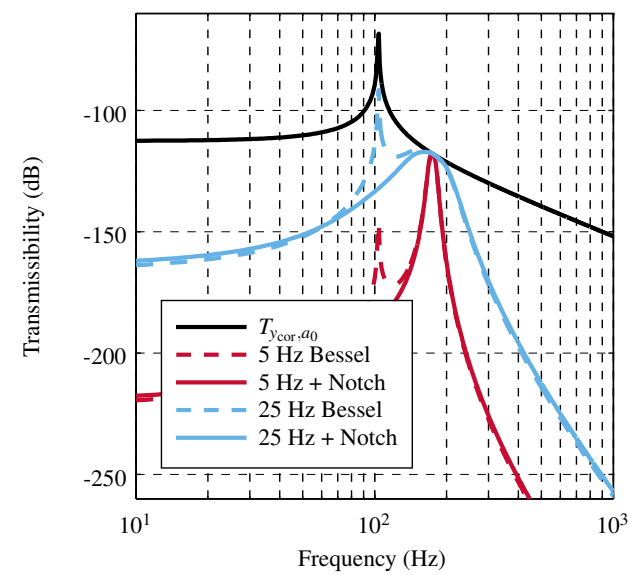

Fig. 6.7 The transmissibility $T_{y_{\text {cor }}, a_{0}}$, unfiltered and with a bandpass filter at the actuation frequency of $170 \mathrm{~Hz}$ and a notch filter at the Coriolis frequency of $96 \mathrm{~Hz}$.

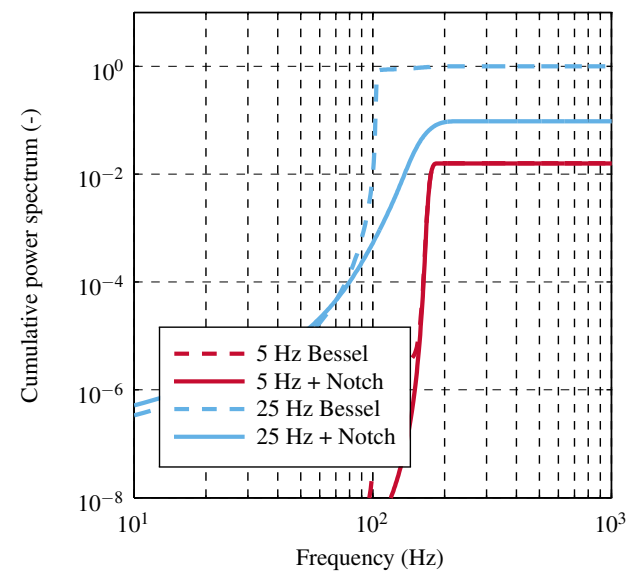

Fig. 6.8 Cumulative power spectrum of the measurement error of the filtered transmissibility of Fig. 6.7 The result is normalised to the maximum value.

The actuation amplitude is kept constant, using feedback control, and the Coriolis displacement is composed of contributions due to the mass-flow, sensor noise, external vibrations. In the remaining of this section, the effect of a mass-flow is omitted.

The quantification of the influence of external vibrations on the RMS measurement value is presented in Chapter 2. Using Parseval's theorem the influence of a Coriolis displacement, uncorrelated with the mass-flow, on the measurement precision can be computed from:

$$
\sigma_{\dot{\Phi}_{\mathrm{m}}}^{2}=\int_{-\infty}^{\infty}\left|\frac{2 F_{\mathrm{LP}}\left(v-\omega_{\mathrm{act}}\right)}{y_{\mathrm{act}} S \omega_{\mathrm{act}}}\right|^{2} \Phi_{y_{\mathrm{cor}}}(\mathrm{v}) d \mathrm{v}
$$

The input is a Power Spectral Density (PSD) $\Phi_{y_{\text {cor }}}$ of the Coriolis displacement, induced by the disturbances. The phase demodulation algorithm, which is explained in section 2.3.2 mixes the sensor signals with the actuation frequency and subsequently filters with a lowpass Bessel filter $F_{\mathrm{LP}}$. The mixing shifts the signal by the actuation frequency and thereby the low-pass filter effectively acts on the effect of disturbances as a band-pass filter around the actuation frequency. The total PSD of the Coriolis displacement due to disturbances is the sum of the effects induced by sensor noise and floor vibrations::

$$
\Phi_{y_{\text {cor }}}=\Phi_{\text {noise }}+\Phi_{\text {vib }}=\Phi_{\text {noise }}+\left|T_{y_{\text {cor }}, a_{0}}(s)\right|^{2} \Phi_{a_{0}}
$$

where $T_{y_{\mathrm{cor}}, \boldsymbol{a}_{0}}(s)$ is the transmissibility of external vibrations $\boldsymbol{a}_{0}$ to the Coriolis displacement. Using Eq. 6.18 and 6.19, the noise level of the CMFM measurement vale is specified as: 


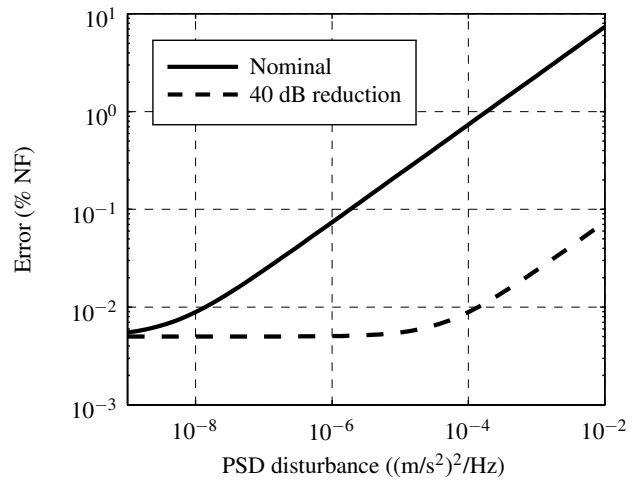

Fig. 6.9 RMS flow error (Eq. 6.20) as function of the external vibrations spectrum amplitude. At least $40 \mathrm{~dB}$ reduction is needed to meet the requirement of $0.01 \% \mathrm{NF}$ at a disturbance level of $1 \mathrm{e}-4\left(\mathrm{~m} / \mathrm{s}^{2}\right)^{2} / \mathrm{Hz}$.

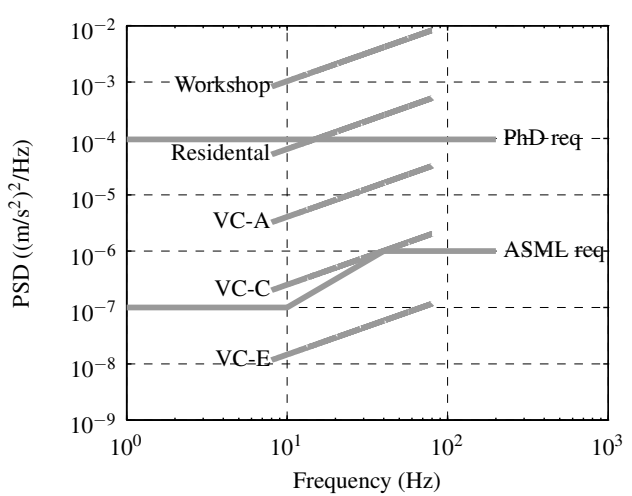

Fig. 6.10 Power Spectral Densities of different floor vibration specifications [23, 55] compared to the requirement of this work.

$$
\begin{aligned}
\sigma_{\dot{\Phi}_{\mathrm{m}}}^{2} & =\sigma_{\text {noise }}^{2}+\sigma_{\mathrm{vib}}^{2} \\
& =\int_{-\infty}^{\infty}\left|\frac{2 F_{\mathrm{LP}}\left(v-\omega_{\mathrm{act}}\right)}{y_{\mathrm{act}} S \omega_{\mathrm{act}}}\right|^{2} \Phi_{\text {noise }}(v) d v+\int_{-\infty}^{\infty}\left|\frac{2 T_{y_{\mathrm{cor}}, \boldsymbol{a}_{0}}(v) F_{\mathrm{LP}}\left(v-\omega_{\mathrm{act}}\right)}{y_{\mathrm{act}} S \omega_{\mathrm{act}}}\right|^{2} \Phi_{a_{0}}(v) d v
\end{aligned}
$$

The measurement error, due to external vibrations, can be approximated by:

$$
\begin{aligned}
\sigma_{\mathrm{vib}} & =\frac{2}{y_{\mathrm{act}} S \omega_{\mathrm{act}}} \sqrt{\int_{-\infty}^{\infty}\left|T_{y_{\mathrm{cor}}, \boldsymbol{a}_{0}}(\mathrm{v}) F_{\mathrm{LP}}\left(\mathrm{v}-\omega_{\mathrm{act}}\right)\right|^{2} \Phi_{\boldsymbol{a}_{0}}(\mathrm{v}) d v} \\
& \approx \frac{2 T_{y_{\mathrm{cor}}, \boldsymbol{a}_{0}}\left(\omega_{\mathrm{act}}\right) \Phi_{\boldsymbol{a}_{0}}^{\frac{1}{2}}\left(\omega_{\mathrm{act}}\right)}{y_{\mathrm{act}} S \omega_{\mathrm{act}}} \bar{F}
\end{aligned}
$$

where $\bar{F}$ is the integrated area under the bandpass filter, including a correction of the approximation due to the -2 slope of $T_{y_{\mathrm{cor}}, a_{0}}$ at $\omega_{\text {act }}$. Further the PSD $\Phi_{a_{0}}$ is assumed to be a flat broadband spectrum, to be able to take it out of the integral. The transmissibility of the approximate 2-DOF model (Eq.6.1) is equal to:

$$
T_{y_{\mathrm{cor}}, a_{0}}(s)=\frac{y_{\mathrm{cor}}(s)}{s^{2} x_{0}(s)}=\frac{-\left(m+\rho_{\mathrm{f}} A_{\mathrm{f}} L\right)}{\left(m+\rho_{\mathrm{f}} A_{\mathrm{f}} L\right) s^{2}+2 k}
$$

The transmissibility function $T_{y_{\text {cor }}, a_{0}}$ is depicted in Fig. 6.7 The sensor signals have a notch filter [16] at the Coriolis frequency before they are used in the phase demodulation algorithm. Combining Eq. 6.7, 6.8, 6.23 and 6.24 result in a RMS mass-flow error, based on model properties: 


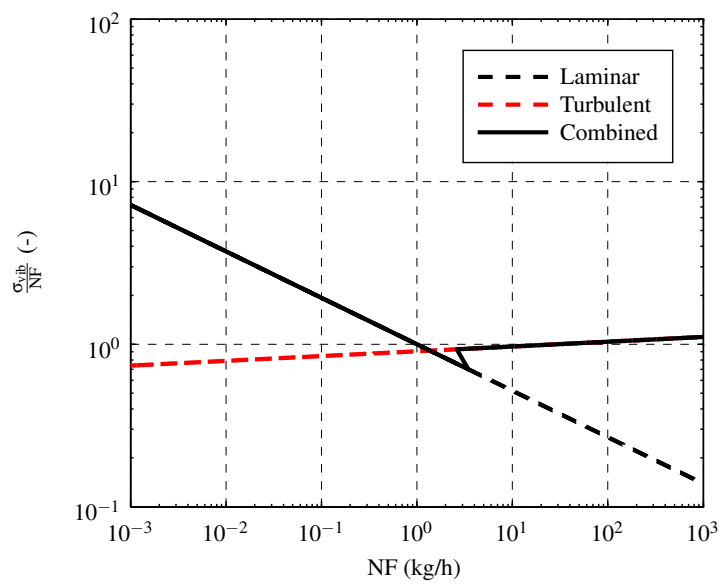

Fig. 6.11 The error percentage of the nominal flow due to external vibrations as function of the nominal flow. The influence of external vibrations increases for a lower flow in the laminar flow regime. The result is normalised on the error belonging to $1 \mathrm{~kg} / \mathrm{h}$.

$$
\sigma_{\mathrm{vib}}=\frac{\bar{F} r\left(m+\rho_{\mathrm{f}} A_{\mathrm{f}} L\right)^{\frac{3}{2}}}{\alpha L y_{\mathrm{act}} \sqrt{2 k}} \Phi_{a_{0}}^{\frac{1}{2}}\left(\omega_{\mathrm{act}}\right)
$$

The result of Eq. 6.20 as function of $\Phi_{a_{0}}$ is depicted in Fig. 6.9, whereby a noise floor of $\sigma_{\text {noise }}=0.005 \% \mathrm{NF}$ is included.

The level of external vibrations is application dependent and realistic values are specified using norms. In Fig. 6.10 several norms are depicted: (i) Vibration Critria (VC) curves [23], (ii) the ASML norm [55], (iii) the requirement within this project. An example of measuring the external vibrations (e.g. floor vibrations) is given in Segerink et al. [56]. The requirement posed in this project is a broadband level of $1 \mathrm{e}-4\left(\mathrm{~m} / \mathrm{s}^{2}\right)^{2} / \mathrm{Hz}$ between 1 and $500 \mathrm{~Hz}$, resulting in no significant influence ( $\max 0.01 \% \mathrm{NF}$, which is comparable to the sensor noise level) of external vibrations on the measurement value. This vibration level is chosen to avoid stringent requirements on the location for installing a CMFM. To have a RMS measurement error of only $0.01 \% \mathrm{NF}$, almost $40 \mathrm{~dB}$ vibration isolation is needed to meet this requirement, as shown in Fig. 6.9 A further reduction of vibrations is desirable, since this allows to increase the filter band, which results in a faster response to flows. A larger frequency band, however, increases the effect of sensor noise and vibrations on the flow, as shown in Fig. 6.8 for noise and vibrations disturbances with a flat PSD input spectrum.

Scaling down the instrument with the same relative effect of noise of the position sensor, requires that $S \cdot \omega_{\text {act }}$ scales inversely with the flow according to Eq.6.21 In the previous subsection 6.1.3, the preferred Coriolis and actuation mode have been chosen to enable this scaling in the low-flow range. Hereby the translational mode of the tube is defined as the Coriolis mode. The downside of this measure is that it makes the CMFM more vulnerable for external vibrations, because there are mainly translational external vibrations. A pure rotational disturbance is rare, but a rotational disturbance somewhere can be seen as a translational dis- 
turbance in a specific node. It is impossible to increase the sensitivity for flow (Eq. 6.7) and decrease the sensitivity for external vibrations (Eq. 6.25) at the same time by changing the Coriolis-frequency, the sensor position, the actuation frequency, the actuation amplitude or the frequency ratio. For a constant sensor position $r / L$, actuation frequency, actuation amplitude and frequency ratio $\alpha$, the sensitivity for external vibrations scales with $\sigma_{\text {vib }} \sim d^{\frac{5}{2}}$. The relative error is depicted in Fig. 6.11, showing an increasing relative sensitivity for vibrations when lowering the flow range.

Focussing on the influence of external vibration, it is thus shown, that for scaling down the instrument's measurement range and preserving the relative sensitivity for displacement sensor noise, the effects of external vibrations becomes dominant in the measurement error. Eq. 6.23 shows that the RMS measurement error depends on the transmissibility $T_{y_{\text {cor }}, a_{0}}$. If the transmissibility function from external vibrations to the Coriolis displacement can be decreased at the actuation frequency, without changing the sensitivity for flow, then this will result in a lower measurement error.

\subsubsection{Conclusions}

Scaling down a CMFM reduces the effect of flow on the ratio between the amplitudes of the actuation and Coriolis motion. Thereby the relative effect of sensor noise on the measurement signal increases. This effect can be reduced by increasing the sensitivity for the Coriolis-motion by switching the actuation and Coriolis-mode. However, this also increases the sensitivity for external vibrations. The relative effect of vibrations thus increases with decreasing the flow capacity. Therefore, the focus of this thesis is on the minimisation of the influence of external vibrations.

\subsection{CMFM designs}

Many CMFM tube designs have been published. In Fig. 6.12 several designs are depicted. A classification of the flow sensor design is presented in Wang and Baker [78]. Single tube designs have a straight or bended design. Twin, or multiple, tube designs are also feasible in straight or bended tube configurations, whereby the flow path is making a single or multiple loop. A multiple tube, single loop requires a flow splitter.

To measure the Coriolis effect, the measurement system must be sensitive to oscillation amplitudes of only a few nanometre. To protect the system from the influence of external vibrations, the system has to be balanced [1].

Conventionally the effect of vibrations is reduced by using a twin tube design. Both tubes respond the same to vibrations but differently to flow, such that the effect of vibrations can be eliminated. It is important that both loops have identical dynamic properties. The amount vibration suppression scales progressively with the frequency mismatch. 

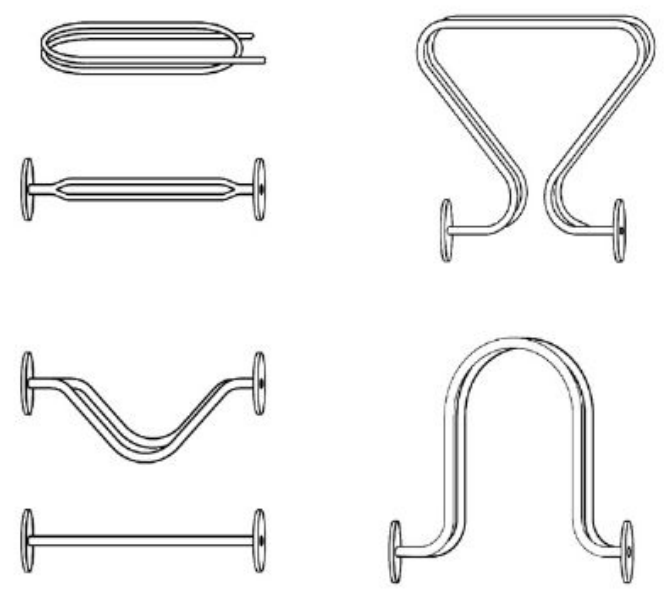

Fig. 6.12 Configurations CMFM tube-shape designs: single and twin tube designs with a single or multiple loop. (Courtesy of Anklin et al. [1])

In our investigation of scaling down the performance of the CMFM, the tube design results in slender tubes with diameters less than a millimetre. In a twin tube design, both tubes are not the same. This is making the design sensitive for external vibrations. Furthermore, the flow sensitivity is different for each tube, while the flow split is unknown. A relative measurement between both tubes is required to annihilate the effects of external vibrations. It is not possible to mount the sensors (and actuators) onto small tubes, because this will increase the mass enormously. Therefore the absolute motion of both tubes must be measured relative to the casing. This, combined with a high Q-factor of the tube, requires a high range of the sensors, which increases the noise. A higher noise results in a higher zero stability of the instrument.

Thus, the twin tube design has several disadvantages when used in a CMFM for very-low flows. Therefore, a single tube design with a high sensitivity for flow is preferred, even though the measurement is also sensitive for external vibrations. This creates the challenge to find a proper balancing mechanism to minimise the influence of external vibrations. In our approach a single tube with a high sensitivity for flow is used and an additional vibration isolation module is developed to lower the sensitivity for external vibrations. Different configurations of such a module are discussed in the next section. 


\subsection{Vibration isolation configurations}

As motivated above, designing a CMFM for low flows, below $1 \mathrm{~kg} / \mathrm{h}$, created new challenges to have an instrument sensitive for flow, but insensitive for external vibrations. To acquire such an instrument, the flow measurement and the vibration isolation part are separated. An overview of all configurations analysed in this thesis is depicted in Fig. 6.13. The main goal is to reduce the transmissibility for external vibrations $a_{0}$ to the Coriolis displacement $y_{\text {cor }}$, without changing the sensitivity for flow, which is the transfer function from $F_{\text {cor }}$ to $y_{\text {cor }}$, evaluated at the actuation frequency. The results of those configurations are presented is the Chapters 2-5 with the objective to reduce the influence of external vibrations by $40 \mathrm{~dB}$. An evaluation of the configurations is presented in the following subsections, prior to an overview of the of advantages and disadvantages summarised in Table 6.1

\subsubsection{Reference instrument}

In Fig. 6.13(a) the model of a reference instrument is depicted, which is the Coriolis mode of the model depicted in Fig. 6.1 and the first Coriolis mode of the model presented in Chapter2. This model comprises the effect of a mass-flow, by means of a force $F_{\text {cor }}$, and the influence of external vibrations $a_{0}$ on the displacement $y_{\text {cor }}$. Using Eq. 6.23 the influence of external vibrations is determined as:

$$
\sigma_{\dot{\Phi}_{\mathrm{m}}} \approx \frac{2 \bar{F}}{y_{\mathrm{act}} S \omega_{\mathrm{act}}} \Phi_{y_{\mathrm{cor}}}^{\frac{1}{2}}\left(\omega_{\mathrm{act}}\right)
$$

where $y_{\text {act }}$ is the actuation amplitude, $\omega_{\text {act }}$ the actuation frequency, $S$ the sensitivity for flow, $\bar{F}$ the low-pass filter in the phase demodulation and $\Phi_{y_{\text {cor }}}$ the PSD of the displacement measurement. The displacement measurement is influenced by the Coriolis force and external vibrations, but also contains sensor noise. Therefore there is also a noise floor in the mass-flow measurement value, independent of the level of external vibrations. The reference instrument of this section is used in subsequent section as a reference for determining the attenuation achievable by various vibration reduction strategies. The attenuation is relative and thus independent of the actuation amplitude and frequency, the sensitivity for flow and the filtering constant. The attenuation is only limited by the sensor noise of the tube displacement sensors.

\subsubsection{Passive vibration isolation - Flexible suspension}

Vibration isolation can be achieved by adding a flexible suspension between the CMFM and the floor, as depicted in Fig. 6.13(b) Examples of flexible suspensions are analysed in Chapter 3 and 4 The model in Fig. 6.13(b) is 1D, though the examples analysed in Chapter 3 and 4 are multi-DOF. The results show MIMO coupling from the external vibrations to the vibra- 


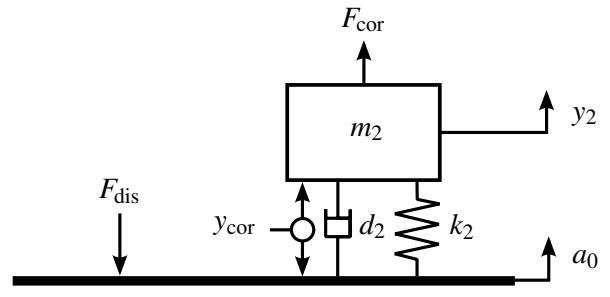

(a) Reference Instrument

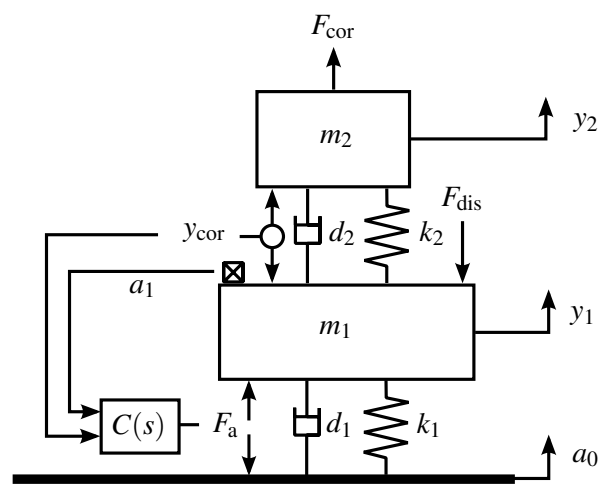

(c) Actively suspended - Feedback

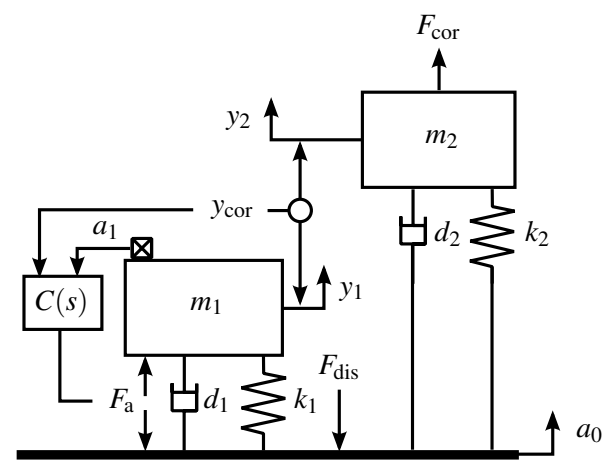

(e) Compliantly mounted sensor

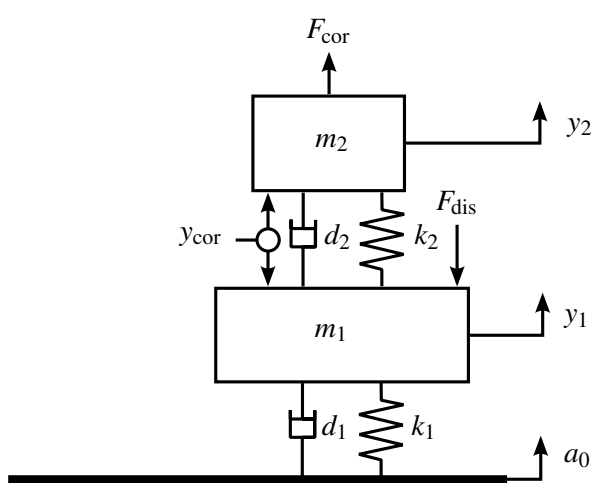

(b) Passively suspended

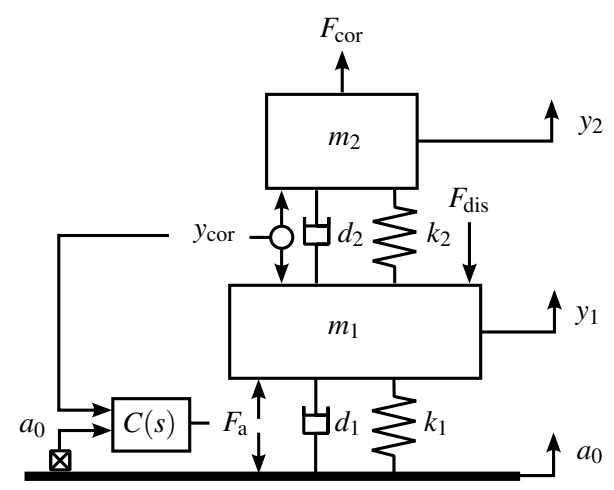

(d) Actively suspended - Feedforward

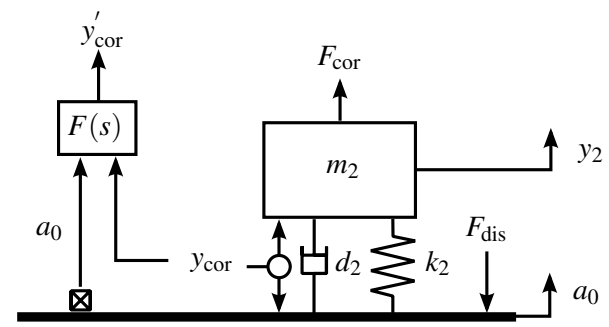

(f) Estimation by filtering

Fig. 6.13 Analysed CMFM configurations with and without vibration isolation. 
tions of the stage. A reduction of the effect of vibrations by a flexible suspension requires an exact constraint design, where the centre of mass and the centre of stiffness are carefully aligned.

Another disadvantage of the flexible suspended stage is the sensitivity for a disturbance force $F_{\text {dis }}$, resulting from the actuation force and acting on the Coriolis direction by asymmetric damping of the stage [68]. This force results in an offset of the measurement value, because $F_{\text {dis }}$ is at the actuation frequency and in phase with the Coriolis Force $F_{\text {cor }}$. Any variation of the damping, e.g., over temperature and time, results in a zero drift of the instrument. This sensitivity can be reduced by increasing the mass $m_{1}$ or by significantly increasing the stiffness around the actuation axis. Further, the mass-flow measurement value is only sensitive for external vibrations in one translational direction and one rotational direction. Rotations only affect the measurement if they result in a translation at a specific point, see section 2.5.1. So only the accelerations of the stage in this direction needs attenuation. Therefore, the number of the DOFs of the stage can be limited to one.

For a relatively undamped system, the attenuation is approximated by (Eq. 3.17):

$$
\mid \text { attenuation } \mid=\frac{\omega_{\text {susp }}^{2}}{\omega_{\text {act }}^{2}}
$$

A large attenuation is achieved by increasing the separation of the suspension and actuation frequency. The actuation frequency is limited from above to obtain sufficient sensitivity for flow (Eq. 6.7) On the other hand, the suspension frequency is limited from below by: (i) the maximum allowable sag due to gravity, (ii) the maximum internal stresses in the connection tubes (iii) the damping of the suspension: a low relative damping and a low suspension frequency require a large dynamic range of tube displacement sensors. Therefore, the maximum attenuation by a flexible suspension is limited. Furthermore, in this design there is no balancing for the actuation force, driving the flow-tube in resonance. However, by scaling down the instrument, also the stiffness of the flow-tube decreases and therefore also the actuation force applied to the environment is limited.

In conclusion, adding a flexible suspension to the measurement stage of a CMFM is a good solution to achieve vibration isolation, but the performance is limited.

\subsubsection{Active vibration isolation control - Feedback}

The performance of flexibly suspended measurement stage is limited, therefore active vibration isolation means are added to the stage. The model is depicted in Fig.6.13(c) an actuator applies a force $F_{\mathrm{a}}$ on the stage $m_{1}$, based on its accelerations. The feedback strategy, validated in Chapter 3 and 4 increases the mass and damping of the stage virtually. This decreases the suspension frequency and thus increases the attenuation of external vibrations, while maintaining the positive effects of the passive support stiffness. The mechanical equivalent of the feed-back controlled system is depicted in Fig. 6.14 for the controller given in Eq. 4.7. The mass $k_{\mathrm{a}}$ lowers the suspension frequency $\omega_{\text {susp }}$ and the sky-hook damper $k_{\mathrm{v}}$ adds damping to 


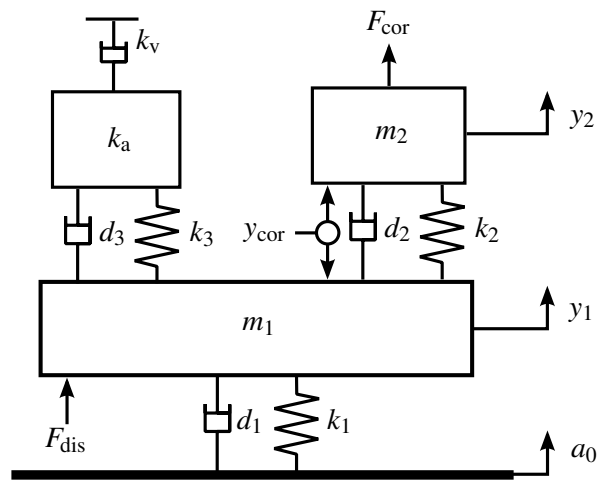

Fig. 6.14 Mechanical equivalent of the CMFM with active vibration feedback control model, depicted in Fig. 6.13(c) The added active means acts as a sky-hook damped balancing mass, suspended at the actuation frequency.

the suspension mode. The parameters $d_{3}$ and $k_{3}$ are chosen such that the added mass $k_{\mathrm{a}}$ acts as a balance mass at the actuation frequency. External vibrations around this frequency would contribute significantly to the measurement error, but the energy of these external vibrations is absorbed by the balancing mass, thus reducing the error. Due to the digital implementation of the controller, the frequency of the balancing mass can be easily kept exactly the actuation frequency. Damping of the Coriolis mode is not added to the mechanical equivalent model, because non-collocated control do not have a physical equivalent.

Adding the active means results in an increased attenuation compared to the passive system. In addition damping is added to the suspension and Coriolis mode, which lowers the required range of the displacement sensors. Furthermore, the effect of the force $F_{\text {dis }}$ is measured by $a_{1}$ and is counteracted by the additional virtual mass.

A potential problem of the feedback strategy are higher order dynamics, which might introduce stability issues. Those issues are solved by using a series of notch filters in the controller. The attenuation can be increased by lowering the suspension frequency, but this is limited because it also increases the bandwidth of the control, making the control schema less robust. Because the higher order dynamics are important for stability, a good design and identification of the system is required. Furthermore, for the strategy, acceleration sensors with low noise are required. For broadband external vibrations with a level of $1 \mathrm{e}-4\left(\mathrm{~m} / \mathrm{s}^{2}\right)^{2} / \mathrm{Hz}$ and $40 \mathrm{~dB}$ attenuation, the required noise floor of the sensors is $1 \mathrm{e}-8\left(\mathrm{~m} / \mathrm{s}^{2}\right)^{2} / \mathrm{Hz}$ or $10 \mu \mathrm{g} / \sqrt{\mathrm{Hz}}$. Such sensors are commercially available, but expensive for small dimensions.

A functional model of a CMFM with active vibration isolation control is validated in Chapter 4 and is depicted in Fig. 6.15. Active vibration isolation based on a feedback strategy is able to provide sufficient attenuation, but requires expensive additional acceleration sensors. 


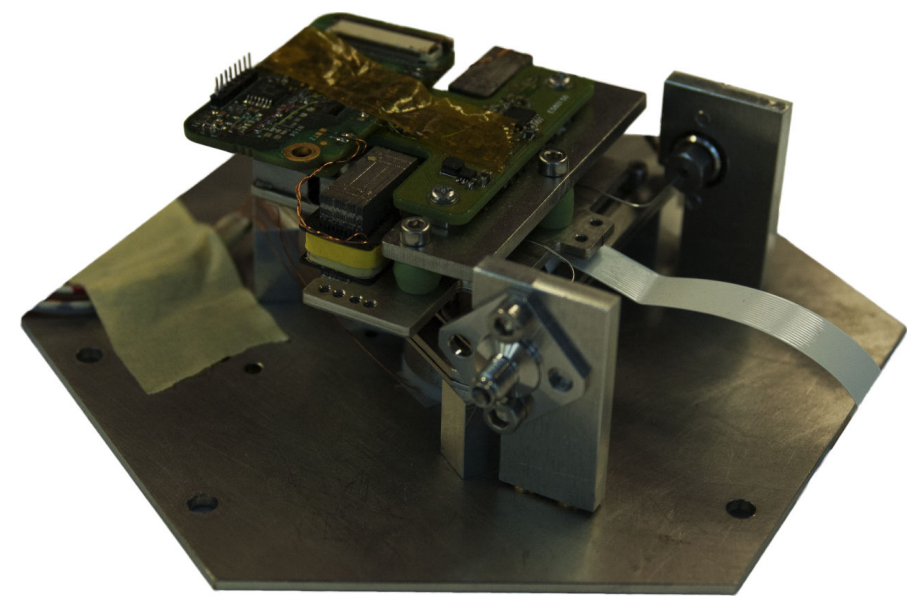

Fig. 6.15 Functional model of of a CMFM with active vibration isolation control. The design is validated in Chapter 4 for the feedback and feedforward strategy.

\subsubsection{Active vibration isolation control - Feedforward}

The limited performance of the flexibly suspended measurement stage can be increased by active vibration isolation means. Alternatively to the feedback strategy, a feedforward strategy can also lower the transmissibility from external vibrations. A model of this strategy is depicted in Fig. 6.13(d), an actuator applies a force $F_{\mathrm{a}}$ on the stage $m_{1}$, based on measured external accelerations $a_{0}$. The feedforward strategy, validated in Chapter 4 can be seen as a cancellation of the forces resulting from the damping and stiffness of the stage's suspension in response to the stage motions.

This strategy cannot add damping to the suspension and Coriolis mode, thus the reduction of the possibly amplitudes of vibrations at the suspension and Coriolis mode frequencies is limited. Therefore the strategy is combined with a feedback controller to damp the suspension and Coriolis mode, based on the Coriolis displacement $y_{\text {cor }}$. This is a control loop with a relatively low bandwidth, compared to the feedback strategy of the previous subsection.

The feedforward strategy is made adaptive in its controller parameters to minimise the error in the presence of changing physical properties. The weights, proportional to the damping, stiffness and actuator induction, are updated using an FxLMS algorithm. This approach is validated using an additional $a_{1}$ error sensor, but modelling results show that $y_{\text {cor }}$, which is already available, is also a good error signal. There is a notch needed at the actuation frequency, because this error signal is also correlated with the Coriolis force $F_{\text {cor }}$ and thus the mass-flow. The notch filter is needed for the adaptation of the weights and also in the feedback controller.

The advantage of the feedforward strategy is that the reference sensors, measuring the external accelerations, can be noisier than the acceleration sensors used in the feedback strategy. For the functional model, sensors with a noise floor of $1 \mathrm{e}-5\left(\mathrm{~m} / \mathrm{s}^{2}\right)^{2} / \mathrm{Hz}$ or $300 \mu \mathrm{g} / \sqrt{\mathrm{Hz}}$

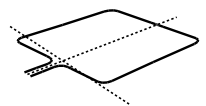


are sufficient (See Fig. 4.10). Further, due to the adaptation of the weights, the attenuation is maximised, independently of the change in the dynamics of the stage. Due to the adaptation only the direct influence of external vibrations, measured by a reference sensor, on the displacement $y_{\text {cor }}$ is compensated for. This makes it possible to use a one-DOF stage with one actuator only, but there are multiple reference sensors needed, because external vibrations in multiple directions can have an influence on the stage translation and thus the measurement value. Another disadvantage is that a disturbance force $F_{\text {dis }}$, uncorrelated with $a_{0}$ is not corrected for.

A functional model of a CMFM with active vibration isolation control is depicted in Fig. 6.15 The same physical experimental setup is used to validate the feedback and adaptive feedforward strategy, as presented in Chapter 4 . Showing that an attenuation of $50 \mathrm{~dB}$ is achievable.

\subsubsection{Compliantly mounted sensor}

Instead of adding a flexible stage between the floor and the measurement stage of a CMFM, a relative measurement of the tube displacements is proposed as well. The model of this second analysed configuration is depicted in Fig.6.13(e) A compliant connection between the casing and the relative motion sensors is added. Tuning the damping and stiffness parameter of this connection, such that the resonance frequency and relative damping are equal to those of the first Coriolis mode, minimises the influence of $a_{0}$ on the displacement $y_{\text {cor }}$. By tuning the dynamic properties of the compliant connection, an external vibration has the same effect on $m_{1}$ and $m_{2}$, therefore the influence is minimised. This solution can be seen as a twin tube design, but with the possibility to connect the sensor to the imaginary tube.

The dynamics of the Coriolis tube are changing, in particular when the density of the fluid is changing. This reduces the achievable attenuation, therefore an active compliantly mounted sensor is proposed. Based on acceleration feedback the resonance frequency is lowered to trace the change of the tube's eigen frequency exactly.

An advantage of this design is that the tube displacement sensors are not influenced by gravitational sagging, because the resonance frequencies of the tube and the mounted sensor are approximately the same. Therefore, the sensors can always be used in the middle of the range.

The performance is limited by the existence of the second Coriolis mode. This limits the attenuation at the actuation frequency. The analysis in Chapter 5 shows that a miss-match of the compliant sensor and the first Coriolis resonance frequency results in an anti-resonance frequency. This miss-match is used to tune the dynamic properties of the compliant connection, such that the anti-resonance frequency occurs at the actuation frequency and thus maximises the achievable attenuation.

A large range of the displacement sensors is needed, because the tube and the sensor suspension has a high Q-factor, resulting in a large displacement when external vibrations are present. This approach only achieves attenuation in a limited frequency range. Therefore, it is 


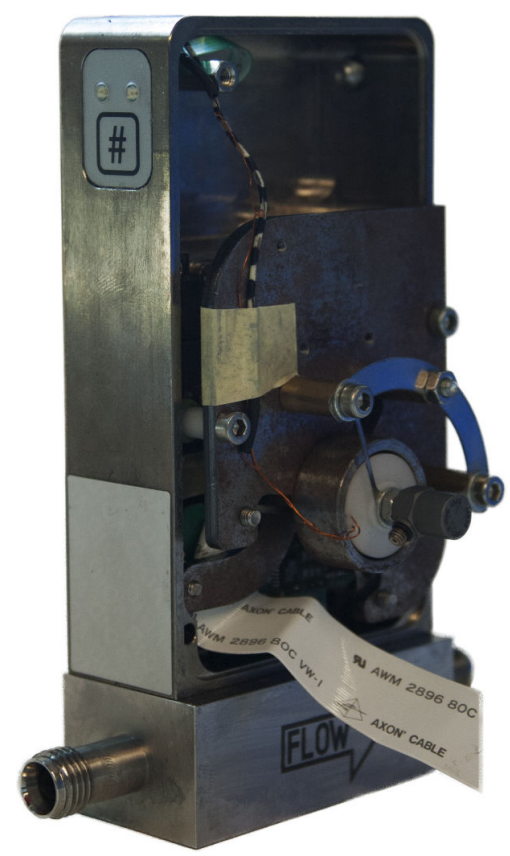

Fig. 6.16 Functional model of a CMFM with an active compliantly mounted sensor. The design is validated in Chapter 5

only useful for instruments with a small low-pass filter in the phase demodulation algorithm and thus a low response time.

A functional model of a CMFM with an active compliantly mounted sensor is depicted in Fig. 6.16 The strategy is validated in Chapter 5 and shows more than $24 \mathrm{~dB}$ reduction of the influence of external vibrations on the mass-flow measurement value. The performance is limited by the second Coriolis mode.

\subsubsection{Estimation by filtering}

Another approach is to use filtering techniques to eliminate the effect of external vibrations from the sensor signals measuring the tube-displacements. Wang and Baker [78] mentioned this in his discussion. In the area of signal processing and control techniques, the application of DSP techniques and powerful hardware allow for on-line correction algorithms and multiparameter measurement. This will improve and extend the Coriolis technology. The approach is investigated by Stroeken [60].

In this approach the measured tube displacements, influenced by external vibrations, are compensated by a measurement of the external vibrations (see Fig. 6.13(f)]. Therefor, a filter

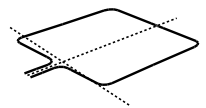


is needed, which is ideally the transmissibily $T_{y_{\mathrm{cor}}, a_{0}}$. The first attempts is using a reduced model, comprising only the first Coriolis mode. The achieved reduction is approximately $20 \mathrm{~dB}$, likely limited by the other Coriolis modes.

The parameters are subjected to variations, due to change of the mass (different fluid densities) and stiffness (due to temperature fluctuations) of the tube. Therefore, a Kalman filter is used for on-line estimation of the transmissibily $T_{y_{\mathrm{cor}}, a_{0}}$. However, for this estimation frequency domain content at the actuation frequency cannot be used, otherwise there will be a correlation with the mass-flow. Instead, the influence of external vibrations on the relatively undamped Coriolis resonance oscillation can be used.

A disadvantage of this approach is that both the model estimation and the correction can be influenced by the Coriolis force $F_{\text {cor }}$, resulting in an erroneous mass-flow measurement value. Another disadvantage of this approach is the necessity of an acceleration sensor with low noise.

\subsubsection{Combinations}

The three analysed configurations, (i) a passive or active flexibly suspended measurement stage, (ii) a relative tube displacement measurement and (iii) estimation by filtering, can be combined to maximise the attenuation. The total achievable attenuation is mainly dependent on the noise levels of all the involved sensors.

\subsection{Conclusions}

This chapter summarises the insights gained in this project. First, the sensitivity to massflow, sensor noise and external vibrations is explained using a simple model of a CMFM. Scaling down the nominal flow range of the instrument results in a smaller diameter and laminar flow. Due to this transition the sensitivity does not scale up proportionally with scaling the flow down. A smaller sensitivity for flow results in a higher sensitivity for sensor noise. Therefore, the actuation and Coriolis mode are switched, making the design more sensitive for flow, but also more sensitive for external vibrations. Different solutions are investigated to apply vibration isolation to a CMFM. An overview of the of advantages and disadvantages is presented in Table 6.1 The solutions consists of passive and active vibration isolation and correction using an on-line model estimator. The insights were tested using two functional models, which are presented in detail in Chapter 4 and 5 (see also Fig. 6.15] and 6.16). They are used to validate the models and show that a reduction in the sensitivity for external vibrations of more than $40 \mathrm{~dB}$ can be achieved. The configurations can be combined and used in the next generation CMFM for very and ultra-low flows. The sensitivity of these meters can be increased to compensate for the reduction of relative sensitivity, while the effect of external vibrations does not grow proportionally by the added vibration isolation. 


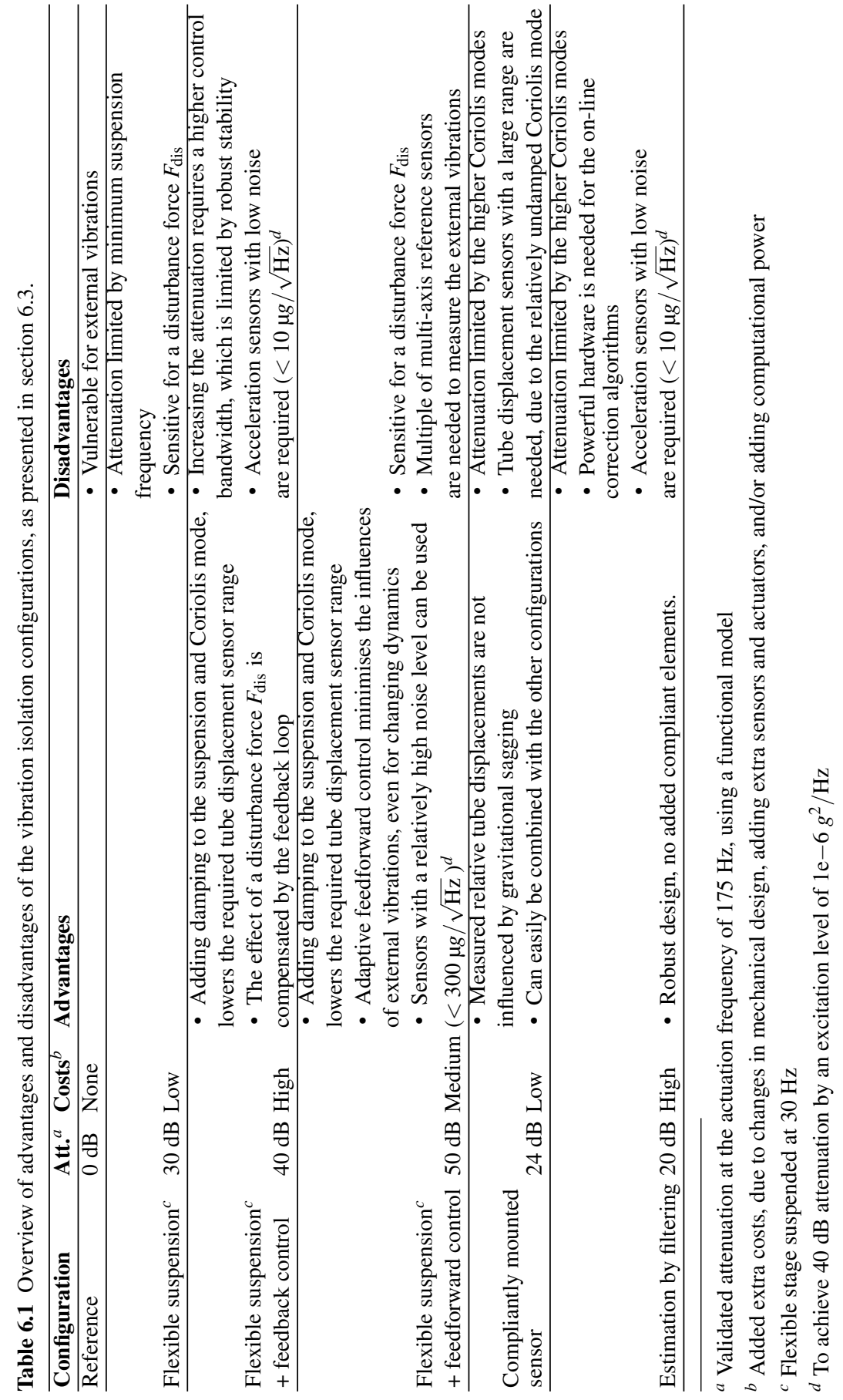




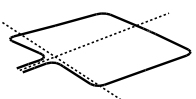




\section{Chapter 7 \\ Conclusions and Recommendations}

\subsection{Conclusions}

The research objective of this thesis was formulated as:

Investigate the effect of external vibrations on the performance of a Coriolis Mass-Flow Meter by quantitative modelling of the influence of external vibrations. Based on these insights, develop methods to reduce the effect of vibrations significantly (factor 100 or $40 \mathrm{~dB}$ ) without changing the performance and validate these conceptual solutions experimentally.

With respect to the objective, it can be concluded that this objective has been achieved. A Coriolis Mass-Flow Meter (CMFM) with integrated vibration isolation control was designed and validated. More specific conclusions regarding the research can be drawn. They are presented below, combined by subject: modelling, methods of vibration isolation, active vibration isolation control strategies, functional models and achieved performance.

Modelling

A flexible 3D multi-body model of a CMFM was developed to understand and quantify the influence of external vibrations on the mass-flow measurement (Chapter 2). The mass-flow is determined using the phase difference measurement and the measurement sensitivity for flow. The phase difference is determined using a phase demodulation algorithm, however in the model it is the ratio between the Coriolis and actuation displacement amplitudes. In an experiment, predefined vibrations has been applied on the casing of the CMFM and the RMS measurement error was determined. The experimental results correspond well on a quantitative level with the modelled results. The result is a significant extension of the work of Cheesewright [13], since it not only shows the frequencies to which the CMFM is sensitive for external vibrations, but it also provides a quantitative estimation of the expected massflow error, based on the modelled transmissibility function. The agreement between model and measurements implies firstly that the influence of any external vibration spectrum on the flow error, with some limitations due to linearity of the model, can be estimated. Thereby, the 
suitability of a certain location for the placement of a CMFM can be determined. Secondly, the insight in the relation between vibration spectra and the flow error, the transmissibility, can be used to compare the performance of different CMFM designs and to optimise the performance by shaping their respective transfer functions.

Besides the sensitivity for vibrations, the model also provides the dominant direction for the influence of external vibrations (Chapter 2). The model shows that there is a percussion point, where only a translational external vibration in one direction results in a significant Coriolis displacement. Therefore, the 3D model can be approximated quite well by a $1 \mathrm{D}$ model. This can be done with a mass-spring model, containing the modal mass and stiffness of the first Coriolis mode.

Scaling down a CMFM reduces the effect of flow on the ratio between the actuation and Coriolis motion amplitudes (Chapter 6.1). This effect can be partly compensated by increasing the relative sensitivity for the Coriolis-motion by switching the actuation and Coriolismode. However, this also increases the sensitivity for external vibrations. The net result is that the relative effect of vibrations increases with decreasing the flow capacity. Thus, to develop a CMFM for low nominal flows $(<1 \mathrm{~kg} / \mathrm{h})$ with a high accuracy and precision, and thus a high sensitivity for flow, extra attention to the influence of external vibrations is required. Therefore, the focus in this thesis was on applying vibration isolation to a CMFM.

\section{Methods of vibration isolation}

Vibration isolation is achieved when attenuation, in a small frequency band in the transmissibility from external vibrations to the Coriolis displacement, is realised. Different methods are elaborated in this work and compared to each other (Chapter 6.3). The vibration isolation has been designed as a module to add to a CMFM, thereby the Coriolis tube design is unaffected. Therefore, the vibration isolation module and tube design can be optimised separately.

The first investigated method adds a flexible suspension between de CMFM tube and the casing (Chapter 3 and 4). This is a form of passive vibration isolation [53]. The performance is insufficient, because the suspension frequency has a lower bound, which limits the attenuation. The suspension frequency is limited to $30 \mathrm{~Hz}$ due to the maximum stress in the connection tubes and a maximum allowable sag of the stage due to gravity. Lowering the transmissibility of external vibrations even more, is achieved by active vibration isolation (Chapter 3 and 4). Therefor extra sensors and actuators are added to the passive system. A feedback and feedforward control strategy has been developed to lower the transmissibility for external vibrations, while maintaining the positive effects of the passive support stiffness. Actively lowering the, relative high, suspension frequency is a form of active hard mount vibration isolation [64, 76], with the distinction that for the application to a CMFM the main goal is not to lower the suspension frequency, but to achieve attenuation in a small frequency band.

The second investigated method adds a compliance between the casing and the tube displacement sensor (Chapter 5). This enables a true displacement measurement of the tube, not influenced by external vibrations. In this novel design, the influence of external vibrations on the compliant sensor element and the tube are made equal by actively tuning the resonance frequency and damping of the compliant sensor element and thereby the influence on the relative displacement measurement is minimised. The effect of the varying internal dynamics due 
to a fluid-density change are addressed in detail and solved by actively tuning the compliant sensor element, using acceleration feedback. Another advantage of the compliantly mounted sensor module is that the influence of the gravitational sagging is minimal. Thereby the displacement sensors are always in the same part of the characteristic sensor response curve, independent of the orientation of the instrument.

The methods to integrate active vibration isolation in a CMFM resulted in functional models (Chapter 4 and 5) and are filed for a patent (Appendix C).

\section{Active vibration isolation control strategies}

Multiple methods has been developed to apply active vibration isolation. A feedback and feedforward strategy are compared in this thesis and both result in a large attenuation of the influence of external vibrations on the measurement value.

A known feedback control strategy [34, 52, 77] is adapted and tuned for integration with a CMFM (Chapter 3). The transmissibility of external vibrations is reduced actively in the region of interest by acceleration feedback. A PI-controller is implemented to lower the suspension frequency and to add skyhook damping. The control bandwidth is limited using a low pass filter with an undamped frequency equal to the actuation frequency of the Coriolis tube, adding extra attenuation around the actuation frequency of the Coriolis tube. The actuation frequency is relatively high, around $170 \mathrm{~Hz}$, resulting in a large control bandwidth. The attenuation, using feedback control, is limited by stability issues induced by higher order stage dynamics and therefore the attenuation is limited. Further, the poorly damped Coriolis mode is damped using positive position feedback of the internal deformation. This feedback loop needs a notch filter on the actuation frequency, to prevent interference of the feedback controller with the mass-flow measurement. The modelling shows for feedback control an achievable attenuation of $46 \mathrm{~dB}$ with a control bandwidth of $300 \mathrm{~Hz}$, whereby the $30 \mathrm{~Hz}$ suspension frequency is actively reduced to $22 \mathrm{~Hz}$.

Secondly, a novel feedforward strategy is developed (Chapter 4). An optimal feedforward controller is derived, which is only dependent on the physical parameters - stiffness, damping and actuator dynamics - between the casing and the suspended stage, no knowledge of the internal dynamics is needed. This can be understood conceptually as follows; by compensating the forces due to the stiffness and damping, no forces are transmitted from the casing to the stage. The controller can be written as a series of Infinite Impulse Response (IIR) filters with ideal parameters. In practice, only constant, estimated parameters are available, while the physical parameters vary. Therefore, a filtered-reference least-mean-square (FxLMS) algorithm is used to determine the optimal feedforward controller weights. The weights are a function of the physical parameters and are online adapted to minimise a squared error signal; the motion of the measurement stage. The IIR filters have fixed poles, this makes the adaptation inherently stable. The huge advantage of this strategy is the limited amount of weights, compared to conventional adaptation strategies. The approach can be used more general as feedfoward control for vibration isolation of precision machines as published elsewhere [4].

The performance of the feedforward controller is limited by the noise levels of the reference and error sensors. The noise level of the reference sensor should be maximal $30 \mathrm{~dB}$ higher than the noise level specification whereby external vibrations are not affecting the mass-flow measurement. This value of $30 \mathrm{~dB}$ is equal to the attenuation achieved by the pas- 
sively suspended stage. For a worthwhile weight adaptation, the ratio between the noise level of the error sensor and the level of external vibrations should be lower than the required attenuation. The modelling shows that for feedforward control, attenuation of more than $50 \mathrm{~dB}$ is achievable.

\section{Functional models}

The methods for vibration isolation were validated experimentally.

The first functional model was used to validate the passive and active vibration isolation methods (Chapter 4). Based on the results obtained from the model a functional model is built. The measurement stage is passively suspended at $30 \mathrm{~Hz}$ in the three out-of-plane directions and active vibration isolation is added to increase the attenuation. The designed stage had more than one dominant direction of influence of external vibrations affecting the measurement value. This can be resolved in future functional models by placing the centre of mass in the centre of compliance, or by lowering the number of degrees-of-freedom of the suspended stage. The active vibration isolation control results in $40 \mathrm{~dB}$ reduction of the influence of external vibrations, without affecting the mass-flow measurement.

The second functional model has been developed to validate the compliantly mounted sensor (Chapter 5). An existing CMFM is extended with the compliantly mounted sensor. Ideally, the influence of external vibrations is equal on a reference mass, comprising the relative tube displacement sensors, and the Coriolis tube. The performance is limited by the second mode in the Coriolis direction. The compliantly mounted sensor has only one intentional mode and this second mode thus results in a different response. The parameters of the compliantly mounted sensor are tuned actively, with acceleration feedback, to have a maximum attenuation, independent of the fluid density. An attenuation of more than $24 \mathrm{~dB}$ is demonstrated for a CMFM with an active compliantly mounted sensor, in case the tube filled with both water and air.

\section{Achieved performance}

The requirement to reduce the effect of vibrations significantly (factor 100 or $40 \mathrm{~dB}$ ) without changing the sensitivity for flow is achieved using a flexibly suspended stage with active vibration isolation.

The required vibration isolation is not achieved for the CMFM integrated with an actively compliantly mounted sensor, because the performance is limited by the second Coriolis mode. However, this method can also be integrated with a passively suspended measurement stage, resulting in an attenuation of over $50 \mathrm{~dB}$.

The achieved reduction is so large that the measurement error for realistic vibration levels is dominated by the noise level of the tube displacement sensors. Further reduction of the performance requires sensors with a (even) lower noise level than currently used. If the noise level of those sensors is reduced by $20 \mathrm{~dB}$, then the performance is limited by the noise of the acceleration sensors used for the vibration isolation strategies. 


\subsection{Recommendations}

A number of recommendations for future research are proposed:

- An on-scale proof of principle is built to validate the modelling. Results show that vibration isolation is achieved. However, the effects of other disturbances should be tested more thoroughly. For example: asymmetry in the instrument, due to temperature difference or dimensions uncertainties can result in a drifting measurement value. The first indicative endurance experiments are showing no significant decrease of the drift performance. The first steps are made to develop a commercially available CMFM with integrated active vibration isolation.

- A validated quantitative model of the influence of external vibrations has been developed (Chapter 2). Some effort is done to use this model to correct the tube displacement measurements to achieve floor vibration isolation [60]. Adding extra sensors to the CMFM, can differentiate the effect of flow and external vibrations on the mass-flow measurement. More research is needed to find the limits of the model based correction approach.

- The adaptive feedforward strategy can be used in many other applications: vibration isolation for precision machines in general [4] or as adaptive feedforward controller for motion control [63].

- For adaptation of the weights of the feedforward controller, an FxLMS algorithm has been used. There are other algorithms available. More research is needed to find the optimal adaptation strategy, with a high conversion rate and low computational costs.

- The compliantly mounted sensor module can also be used in combination with the other vibration reduction techniques, e.g. passive suspension between the casing and the surroundings [72] or extended with active vibration isolation control [70], to increase the reduction even more.

- Vibration isolation is only achieved in a small frequency band around the actuation frequency. Increasing this attenuation band offers the possibility to increase the response time of flow measurement for equal influence of external vibrations. Therefore, the active vibration isolation control strategies need to be improved, to obtain attenuation in a larger frequency band.

- The noise level of the CMFM measurement is dependent on sensor noise levels and the amount of external vibrations (Chapter 6.1.4). Vibration isolation techniques reduce the effects of external vibrations, thus making the influence of the sensor noise dominant. To lower the noise level of the CMFM measurement even more, the sensitivity for flow have to be increased, even though this is increasing the effects of external vibrations. A consideration between the measurement noise, the response time and the vibration sensitivity should be made. 
- In this thesis, different vibration isolation strategies has been developed. The choice for the optimal strategy for further developments is dependent on: achievable attenuation, costs, dimensions and other disturbances effects. The modelling methods of this work, can be used to minimise the effects of external vibrations on the mass-flow measurement, while optimising its performance. 


\section{References}

[1] M. Anklin, W. Drahm, and A. Rieder. Coriolis mass flowmeters: Overview of the current state of the art and latest research. Flow Measurement and Instrumentation, 17(6):317 323, 2006. ISSN 0955-5986. URL http://dx.doi.org/10.1016/j.flowmeasinst. 2006.07 .004 .

[2] J. Anthonis and H. Ramon. Linear mechanical systems and dyadic transfer function matrices. Automatica, 39(8):1353 - 1363, 2003. URL http: //dx.doi.org/10.1016/ S0005-1098 (03) 00107-9.

[3] R.C. Baker. Flow Measurement Handbook. Cambridge University Press, 2000. ISBN 9780511471100. URL http://dx.doi.org/10.1017/CB09780511471100. Cambridge Books Online.

[4] M. A. Beijen, J. Van Dijk, W. B. J. Hakvoort, and M. Heertjes. Self-tuning feedforward control for active vibration isolation of precision machines. In 19th World Congress of the International Federation of Automatic Control (IFAC), 2014. URL http://dx. doi.org/10.3182/20140824-6-ZA-1003.01208.

[5] G. Bobovnik, J. Kutin, N. Mole, B. Štok, and I. Bajsić. Numerical analysis of installation effects in coriolis flowmeters: A case study of a short straight tube full-bore design. Flow Measurement and Instrumentation, 34(0):142 - 150, 2013. ISSN 0955-5986. URL http://dx.doi.org/10.1016/j.flowmeasinst.2013.10.004.

[6] G. Bobovnik, J. Kutin, N. Mole, B. Štok, and I. Bajsić. Numerical analysis of installation effects in coriolis flowmeters: Single and twin tube configurations. Flow Measurement and Instrumentation, 2014. ISSN 0955-5986. URL http://dx.doi.org/ 10.1016/j.flowmeasinst.2014.10.001.

[7] M. Bouchard and S. Quednau. Multichannel recursive-least-square algorithms and fasttransversal-filter algorithms for active noise control and sound reproduction systems. Speech and Audio Processing, IEEE Transactions on, 8(5):606-618, Sep 2000. ISSN 1063-6676. URL http://dx.doi.org/10.1109/89.861382.

[8] Bronkhorst CORI-TECH. Mini cori-flow compact coriolis mass flow meters \& controlers for liquids and gases, 2015. URL http://www.bronkhorst.com/files/br_ coritech/downloads/brochures-en/mini_coriflow.pdf.

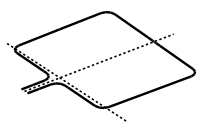


[9] Bronkhorst HIGH-TECH. Application notes, 2015. URL http://www.bronkhorst. com/en/downloads/application_notes/.

[10] H. Butler. Acceleration feedback in a lithographic tool. Control Engineering Practice, 20(4):453 - 464, 2012. ISSN 0967-0661. URL http://dx.doi.org/10.1016/j. conengprac.2011.12.008.

[11] R. Cheesewright and S. Shaw. Uncertainties associated with finite element modelling of coriolis mass flow meters. Flow Measurement and Instrumentation, 17(6):335 347, 2006. ISSN 0955-5986. URL http://dx.doi.org/10.1016/j.flowmeasinst. 2006.07 .003$.

[12] R. Cheesewright, C. Clark, and D. Bisset. The identification of external factors which influence the calibration of coriolis massflow meters. Flow Measurement and Instrumentation, 11(1):1 - 10, 2000. ISSN 0955-5986. URL http://dx.doi.org/10.1016/ S0955-5986(99)00023-0.

[13] R. Cheesewright, A. Belhadj, and C. Clark. Effect of mechanical vibrations on coriolis mass flow meters. Journal of Dynamic Systems, Measurement, and Control, 125(1): 103-113, 2003. URL http://dx.doi.org/10.1115/1.1539098.

[14] C. Clark and R. Cheesewright. The influence upon coriolis mass flow meters of external vibrations at selected frequencies. Flow Measurement and Instrumentation, 14(1-2):33 - 42, 2003. ISSN 0955-5986. URL http://dx.doi.org/10.1016/ S0955-5986(02)00065-1.

[15] G. Coriolis. Sur les équations du mouvement relatif des systèmes de corps. J. de l'Ecole Royale Polytechnique, 15:144-154, 1835.

[16] H. V. Derby, T. Bose, and S. Rajan. Method and apparatus for adaptive line enhancement in coriolis mass flow meter measurement, US5555190, 10 September 1996.

[17] S.J. Elliott, I. Stothers, and P.A. Nelson. A multiple error lms algorithm and its application to the active control of sound and vibration. Acoustics, Speech and Signal Processing, IEEE Transactions on, 35(10):1423-1434, Oct 1987. ISSN 0096-3518. URL http://dx.doi.org/10.1109/TASSP.1987.1165044.

[18] S. Enz, J. J. Thomsen, P. Møller, and L. Davidsen. Factors Affecting Coriolis Flowmeter Accuracy, Precision, and Robustness. DCAMM Special Report. Technical University of Denmark (DTU), 2010. ISBN 9788790416386. URL http:// findit.dtu.dk/en/ catalog/112377959.

[19] S. Enz, J. J. Thomsen, and S. Neumeyer. Experimental investigation of zero phase shift effects for coriolis flowmeters due to pipe imperfections. Flow Measurement and Instrumentation, 22(1):1 - 9, 2011. ISSN 0955-5986. URL http://dx.doi.org/10. $1016 / j$. flowmeasinst.2010.10.002.

[20] Flow Research Inc. The world market for coriolis flowmeters, 4th edition. Technical report, 2013. URL http: //www.researchandmarkets.com/reports/2963653/ the-world-market-for-coriolis-flowmeters-4th.

[21] G. Fowles and W. H. Boyes. Chapter 6 - measurement of flow. In Walt Boyes, editor, Instrumentation Reference Book (Fourth Edition), pages 31 - 68. ButterworthHeinemann, Boston, fourth edition edition, 2010. ISBN 978-0-7506-8308-1. URL http://dx.doi.org/10.1016/B978-0-7506-8308-1.00006-1. 
[22] T. Gerkema and L. Gostiaux. A brief history of the coriolis force. Europhysics News, 43(2):14-17, 2012. URL http://dx.doi.org/10.1051/epn/2012202.

[23] C. G. Gordon. Generic vibration criteria for vibration-sensitive equipment. In C. G. Gordon, editor, Society of Photo-Optical Instrumentation Engineers (SPIE) Conference Series, volume 1619 of Presented at the Society of Photo-Optical Instrumentation Engineers (SPIE) Conference, pages 71-85, February 1992.

[24] J. Groenesteijn, L. van de Ridder, J. C. Lötters, and R. J. Wiegerink. Modelling of a micro coriolis mass flow sensor for sensitivity improvements. In Proceedings of the Thirteenth IEEE Sensors Conference, IEEE Sensors 2014, Valencia, Spain, pages 954957, Valencia, Spain, 2-5 November 2014. IEEE Service Center. URL http:// doc. utwente.nl/92825/.

[25] W. B. J. Hakvoort, J. P. Meijaard, R. G. K. M. Aarts, J. B. Jonker, and J. M. Zwikker. Modeling a coriolis mass flow meter for shape optimization. In The 1st Joint International Conference on Multibody System Dynamics, 2010.

[26] W. B. J. Hakvoort, J. P. Meijaard, and J. C. Lötters. Scaling down coriolis-based mass flow rate sensing. In 12th euspen International Conference, pages 495-498, Stockholm, Sweden, June 2012.

[27] J. Haneveld, T. S. J. Lammerink, M. J. de Boer, R. G. P. Sanders, A. Mehendale, J. C. Lötters, M. Dijkstra, and R. J. Wiegerink. Modeling, design, fabrication and characterization of a micro coriolis mass flow sensor. Journal of Micromechanics and Microengineering, 20(12):125001, 2010. URL http://dx.doi.org/10.1088/0960-1317/20/ $12 / 125001$.

[28] J. Hemp. Calculation of the sensitivity of a straight tube coriolis mass flowmeter with free ends. Flow Measurement and Instrumentation, 12(5-6):411 - 420, 2002. ISSN 0955-5986. URL http://dx.doi.org/10.1016/S0955-5986(01) 00031-0.

[29] M. Henry, M. Tombs, M. Zamora, and F. Zhou. Coriolis mass flow metering for three-phase flow: A case study. Flow Measurement and Instrumentation, 30(0):112 122, 2013. ISSN 0955-5986. URL http://dx.doi.org/10.1016/j.flowmeasinst. 2013.01 .003 .

[30] J. B. Hopkins and M. L. Culpepper. Synthesis of precision serial flexure systems using freedom and constraint topologies (fact). Precision Engineering, 35(4):638 - 649, 2011. ISSN 0141-6359. URL http://dx.doi.org/10.1016/j.precisioneng.2011.04. 006.

[31] J. B. Jonker. A finite element dynamic analysis of spatial mechanisms with flexible links. Computer Methods in Applied Mechanics and Engineering, 76: 17 - 40, 1989. URL http://www.sciencedirect.com/science/article/pii/ 0045782589901394.

[32] J. B. Jonker, R. G. K. M. Aarts, and J. van Dijk. A linearized input-output representation of flexible multibody systems for control synthesis. Multibody System Dynamics, 21:99-122, 2009. ISSN 1384-5640. URL http://dx.doi.org/10.1007/ S11044-008-9130-6.

[33] J.B. Jonker and J.P. Meijaard. Spacar - computer program for dynamic analysis of flexible spatial mechanisms and manipulators. In Werner Schiehlen, editor, Multibody

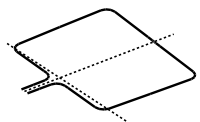


Systems Handbook, pages 123-143. Springer Berlin Heidelberg, 1990. ISBN 978-3642-50997-1. URL http://dx.doi.org/10.1007/978-3-642-50995-7_9.

[34] D. Karnopp and A. Trikha. Comparative study of optimization techniques for shock and vibration isolation. Transactions of American Society of Mechanical Engineers, Journal of Engineering for Industry, 91(4):1128-1132, 1969.

[35] M. Kazahaya. A mathematical model and error analysis of coriolis mass flowmeters. Instrumentation and Measurement, IEEE Transactions on, 60(4):1163-1174, April 2011. ISSN 0018-9456. URL http://dx.doi.org/10.1109/TIM.2010.2086691.

[36] S. M. Kuo and D. Morgan. Active Noise Control Systems: Algorithms and DSP Implementations. John Wiley \& Sons, Inc., New York, NY, USA, 1st edition, 1995. ISBN 0471134244.

[37] J. Kutin and I. Bajsić. An analytical estimation of the coriolis meter's characteristics based on modal superposition. Flow Measurement and Instrumentation, 12 (5-6):345 - 351, 2002. ISSN 0955-5986. URL http://dx.doi.org/10.1016/ S0955-5986(02)00006-7.

[38] I. D. Landau, M. Alma, and T. Airimitoaie. Adaptive feedforward compensation algorithms for active vibration control with mechanical coupling. Automatica, 47(10):2185 2196, 2011. ISSN 0005-1098. URL http://dx. doi.org/10.1016/j.automatica. 2011.08 .015 .

[39] J. C. Lötters, L. van de Ridder, W. D. Kruijswijk, W. B. J. Hakvoort, and M. R. Katerberg. Coriolis flow meter with active vibration isolation, Patent pending, 25 November 2013.

[40] J. C. Lötters, L. van de Ridder, W. D. Kruijswijk, W. B. J. Hakvoort, and M. R. Katerberg. Coriolis flow meter with passive vibration isolation, Patent pending, $24 \mathrm{March}$ 2014.

[41] A.G.J. MacFarlane. Commutative controller: a new technique for the design of multivariable control systems. Electronics Letters, 6(5):121-123, March 1970. ISSN 00135194. URL http://dx.doi.org/10.1049/el:19700083.

[42] F. Matichard, B. Lantz, K. Mason, R. Mittleman, B. Abbott, S. Abbott, E. Allwine, S. Barnum, J. Birch, S. Biscans, D. Clark, D. Coyne, D. DeBra, R. DeRosa, S. Foley, P. Fritschel, J.A. Giaime, C. Gray, G. Grabeel, J. Hanson, M. Hillard, J. Kissel, C. Kucharczyk, A. Le Roux, V. Lhuillier, M. Macinnis, B. O’Reilly, D. Ottaway, H. Paris, M. Puma, H. Radkins, C. Ramet, M. Robinson, L. Ruet, P. Sareen, D. Shoemaker, A. Stein, J. Thomas, M. Vargas, and J. Warner. Advanced LIGO two-stage twelve-axis vibration isolation and positioning platform. part 1: Design and production overview. Precision Engineering, 40:273 - 286, 2015. ISSN 0141-6359. URL http: // dx.doi.org/10.1016/j.precisioneng.2014.09.010.

[43] F. Matichard, B. Lantz, K. Mason, R. Mittleman, B. Abbott, S. Abbott, E. Allwine, S. Barnum, J. Birch, S. Biscans, D. Clark, D. Coyne, D. DeBra, R. DeRosa, S. Foley, P. Fritschel, J.A. Giaime, C. Gray, G. Grabeel, J. Hanson, M. Hillard, J. Kissel, C. Kucharczyk, A. Le Roux, V. Lhuillier, M. Macinnis, B. O’Reilly, D. Ottaway, H. Paris, M. Puma, H. Radkins, C. Ramet, M. Robinson, L. Ruet, P. Sareen, D. Shoemaker, A. Stein, J. Thomas, M. Vargas, and J. Warner. Advanced LIGO two-stage twelve-axis vibration isolation and positioning platform. part 2: Experimental investiga- 
tion and tests results. Precision Engineering, 40(0):287 - 297, 2015. ISSN 0141-6359. URL http://dx.doi.org/10.1016/j.precisioneng.2014.11.010.

[44] A. Mehendale. Coriolis mass flow rate meters for low flows. PhD thesis, University of Twente, Enschede, October 2008. URL http://doc.utwente.nl/60164/.

[45] A. Mehendale, J. C. Lötters, and J. M. Zwikker. Mass flowmeter of the coriolis type, EP1719982, 2 May 2006.

[46] A. Mehendale, J. C. Lötters, and J. M. Zwikker. Coriolis mass flow meter using contactless excitation and detection, EP1719983, 2 May 2006.

[47] J. P. Meijaard. Fluid-conveying flexible pipes modeled by large-deflection finite elements in multibody systems. Journal of Computational and Nonlinear Dynamics, 9: 011008-1-7, 2013. URL http://dx.doi.org/10.1115/1.4025353.

[48] J. P. Meijaard and W. B. J. Hakvoort. Modelling of fluid-conveying flexible pipes in multibody systems. In 7th EUROMECH Solid Mechanics Conference, 2009.

[49] D.R. Morgan. An analysis of multiple correlation cancellation loops with a filter in the auxiliary path. In Acoustics, Speech, and Signal Processing, IEEE International Conference on ICASSP '80, volume 5, pages 457-461, Apr 1980. URL http://dx. doi.org/10.1109/ICASSP.1980.1170986.

[50] D. H. Owens. Feedback and Multivariable Systems (IEE control engineering series). Southgate House, 1978.

[51] M. P. Païdoussis. Slender Structures and Axial Flow, volume 1. Academic Press, New York, 1998. URL http://www.sciencedirect.com/science/bookseries/ $18745652 / 1$.

[52] A. Preumont. Vibration Control of Active Structures. Springer Netherlands, 2011. URL http://dx.doi.org/10.1007/978-94-007-2033-6. ISBN 978-94-007-2032-9.

[53] E. I. Rivin. Passive Vibration Isolation. ASME Press, New York, 2003. ISBN 079810187X.

[54] H. Röck and F. Koschmieder. Model-based phasor control of a coriolis mass flow meter $(\mathrm{cmfm})$ for the detection of drift in sensitivity and zero point. In SubhasChandra Mukhopadhyay, GourabSen Gupta, and RayYueh-Min Huang, editors, Recent Advances in Sensing Technology, volume 49 of Lecture Notes in Electrical Engineering, pages 221-240. Springer Berlin Heidelberg, 2009. ISBN 978-3-642-00577-0. URL http: // dx.doi.org/10.1007/978-3-642-00578-7_13.

[55] R. M. Schmidt, G. Schitter, and J. van Eijk. The Design of High Performance Mechatronics: High-Tech Functionality by Multidisciplinary System Integration. Delft University Press, 2011. ISBN 9781607508267. URL http://ebooks.iospress.nl/book/ the-design-of-high-performance-mechatronics.

[56] F. B. Segerink, J. P. Korterik, and H. L. Offerhaus. Vibration transfers to measure the performance of vibration isolated platforms on site using background noise excitation. Review of Scientific Instruments, 82(6):065111, 2011. URL http://doc.utwente. nl/85322/.

[57] H. M. J. R. Soemers. Design Principles for Precision Mechanisms. T-Pointprint, Enschede, 2010. ISBN 978903653103.

[58] K. Staman. A multi-dof active vibration isolation setup for a coriolis mass flow rate meter. Master's thesis, University of Twente, October, 162014.

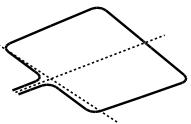


[59] K. Staman, L. van De Ridder, W. B. J. Hakvoort, D. M. Brouwer, and J. van Dijk. A multi-dof active vibration isolation setup for a coriolis mass-flow meter. In 29th Annual Meeting of the American Society for Precision Engineering, Boston, Massachusetts USA, 9-14 November 2014. URL http://doc . utwente.nl/93719/.

[60] K. L. J. Stroeken. Floor vibration isolation of a coriolis mass-flow meter using filtering techniques. Master's thesis, University of Twente, November, 21 2014. URL http:// essay.utwente.nl/66292/.

[61] G. Sultan and J. Hemp. Modelling of the coriolis mass flowmeter. Journal of Sound and Vibration, 132(3):473 - 489, 1989. ISSN 0022-460X. URL http://dx. doi.org/ $10.1016 / 0022-460 \mathrm{X}(89) 90640-8$.

[62] J. J. Thomsen and J. Dahl. Analytical predictions for vibration phase shifts along fluidconveying pipes due to coriolis forces and imperfections. Journal of Sound and Vibration, 329(15):3065 - 3081, 2010. ISSN 0022-460X. URL http://dx.doi.org/10. $1016 / j \cdot j s v .2010 .02 .010$.

[63] J. Tian. Mimo adaptive feed-forward controller design for motion control systems. Master's thesis, University of Twente, 22 August 2014.

[64] D. Tjepkema. Active hard mount vibration isolation for precision equipment. $\mathrm{PhD}$ thesis, University of Twente, Enschede, 2012. URL http://doc . utwente.n1/82175/.

[65] D. Tjepkema, J. van Dijk, and H. M. J. R. Soemers. Sensor fusion for active vibration isolation in precision equipment. Journal of Sound and Vibration, 331(4):735 - 749, 2012. ISSN 0022-460X. URL http://dx.doi.org/10.1016/j.jsv.2011.09.022.

[66] D. Vaes, J. Swevers, and P. Sas. Optimal decoupling for mimo-controller design with robust performance. In Proceedings of the American Control Conference, volume 5, pages 4601-4606, June 2004.

[67] L. van de Ridder. Analysis of a coriolis mass flow meter to reduce influence of external disturbances. Master's thesis, June 222011.

[68] L. van de Ridder, W. B. J. Hakvoort, J. van Dijk, and J. C. Lötters. Influence of external damping on phase difference measurement of a coriolis mass-flow meter. In EUROMECH Colloquium 524, Enschede, 27-29 February 2012. URL http://doc. utwente.nl/89167/.

[69] L. van de Ridder, W. B. J. Hakvoort, J. van Dijk, J. C. Lötters, and A. de Boer. Quantitative estimation of the influence of external vibrations on the measurement error of a coriolis mass-flow meter. In Z. Dimitrovová, J.R. de Almeida, and R. Gonçalves, editors, 11th International Conference on Vibration Problems (ICOVP-2013), Lisbon, Portugal, 9-12 September 2013. URL http://doc. utwente.nl/89168/.

[70] L. van de Ridder, M. A. Beijen, W. B. J. Hakvoort, J. van Dijk, J. C. Lötters, and A. de Boer. Active vibration isolation feedback control for coriolis mass-flow meters. Control Engineering Practice, 33C:76-83, 2014. URL http://dx. doi.org/10. 1016/j.conengprac.2014.09.007.

[71] L. van de Ridder, W. B. J. Hakvoort, and J. van Dijk. Vibration isolation by compliant sensor mounting applied to a coriolis mass-flow meter. In ASME 2014 12th biennial conference on engineering systems design and analysis (ESDA2014), Copenhagen, Denmark, 25-27 June 2014. URL http: // doc . utwente.nl/91292/. 
[72] L. van de Ridder, W. B. J. Hakvoort, J. van Dijk, J. C. Lötters, and A. de Boer. Quantification of the influence of external vibrations on the measurement error of a coriolis mass-flow meter. Flow Measurement and Instrumentation, 40C:39-49, 2014. URL http://dx.doi.org/10.1016/j.flowmeasinst.2014.08.005.

[73] L. van de Ridder, W. B. J. Hakvoort, D. M. Brouwer, J. van Dijk, J. C. Lötters, and A. de Boer. Coriolis mass-flow meter with integrated multi-dof active vibration isolation. 2015. Submitted to Mechatronics (Elsevier).

[74] L. van de Ridder, W. B. J. Hakvoort, and J. van Dijk. Active vibration isolation control: comparison of feedback and feedforward control strategies applied to coriolis mass-flow meters. In ACC 2015 The American Control Conference, Chicago, IL, USA, 1-3 July 2015. URL http://doc.utwente.nl/95710/.

[75] L. van de Ridder, W. B. J. Hakvoort, J. van Dijk, J. C. Lötters, and A. de Boer. Vibration isolation by an actively compliantly mounted sensor applied to a coriolis mass-flow meter. 2015. Submitted to Dynamic Systems, Measurement and Control (ASME).

[76] G. W. van der Poel. An exploration of active hard mount vibration isolation for precision equipment. $\mathrm{PhD}$ thesis, University of Twente, Enschede, 2010. URL http://doc. utwente.nl/71347/.

[77] J. van Dijk. Mechatronic design of hard-mount concepts for precision equipment. In Heinz Ulbrich and Lucas Ginzinger, editors, Motion and Vibration Control, pages 315324. Springer Netherlands, 2009. ISBN 978-1-4020-9437-8. URL http://dx. doi. org/10.1007/978-1-4020-9438-5_31.

[78] T. Wang and R. C Baker. Coriolis flowmeters: a review of developments over the past 20 years, and an assessment of the state of the art and likely future directions. Flow Measurement and Instrumentation, 40:99 - 123, 2014. ISSN 0955-5986. URL http: // dx.doi.org/10.1016/j.flowmeasinst.2014.08.015.

[79] E. Wernholt and S. Gunnarsson. Analysis of methods for multivariable frequency response function estimation in closed loop. In Decision and Control, 2007 46th IEEE Conference on, pages 4881-4888, Dec 2007. URL http://dx.doi.org/10.1109/ CDC.2007.4434780.

[80] R. B. White. Coriolis mass flowmeter, US2832218, 29 April 1958. 


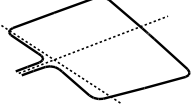


Part II

Appendices

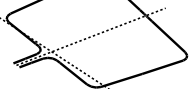




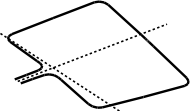




\section{Appendix A \\ Commercially available CMFMs}

In Table A.1 an overview is given of the commercially available CMFMs. Only the low flow range CMFMs are listed completely. A subset is depicted in Fig. A.1

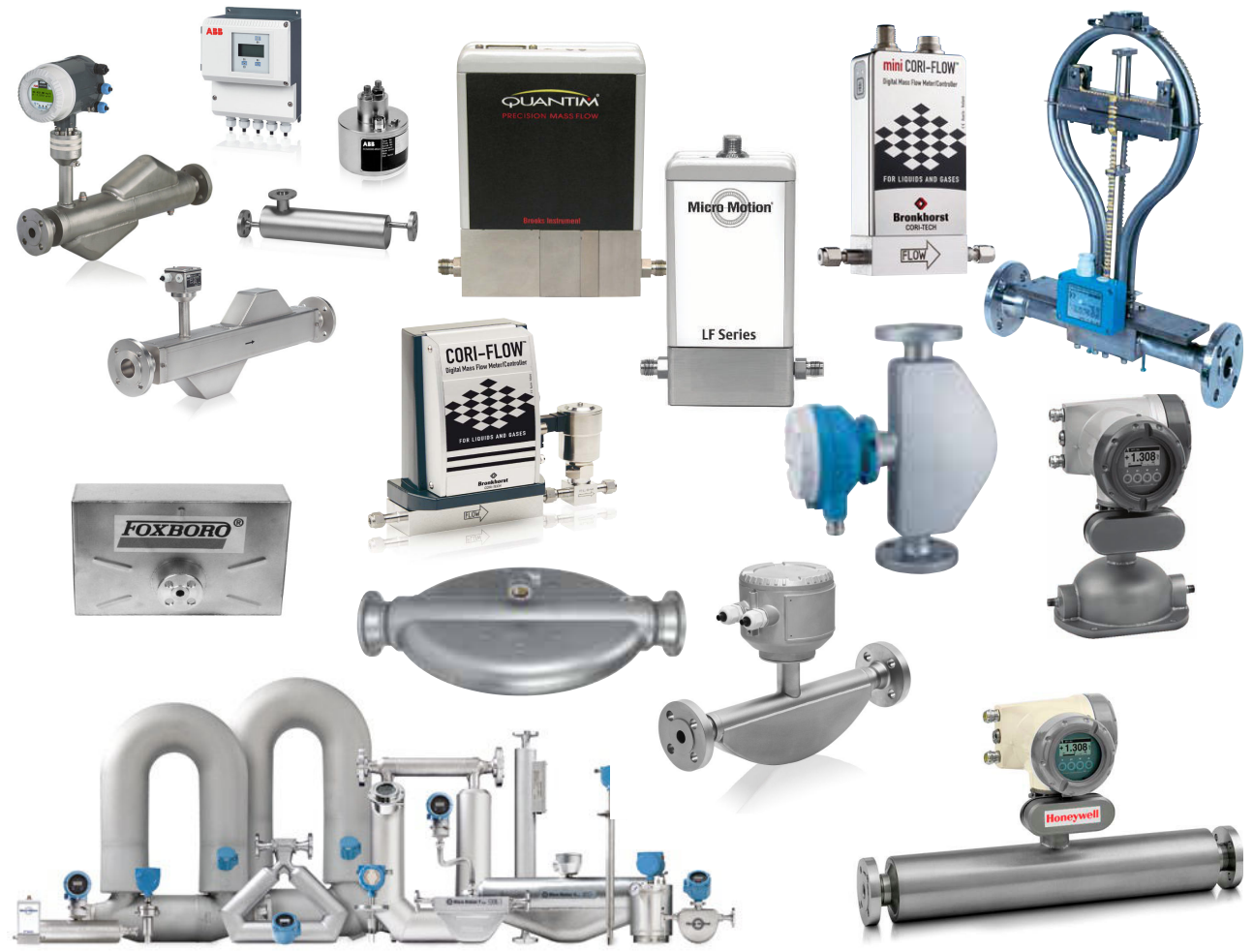

Fig. A.1 Set of commercially available CMFMs

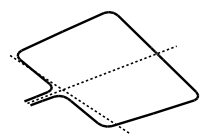




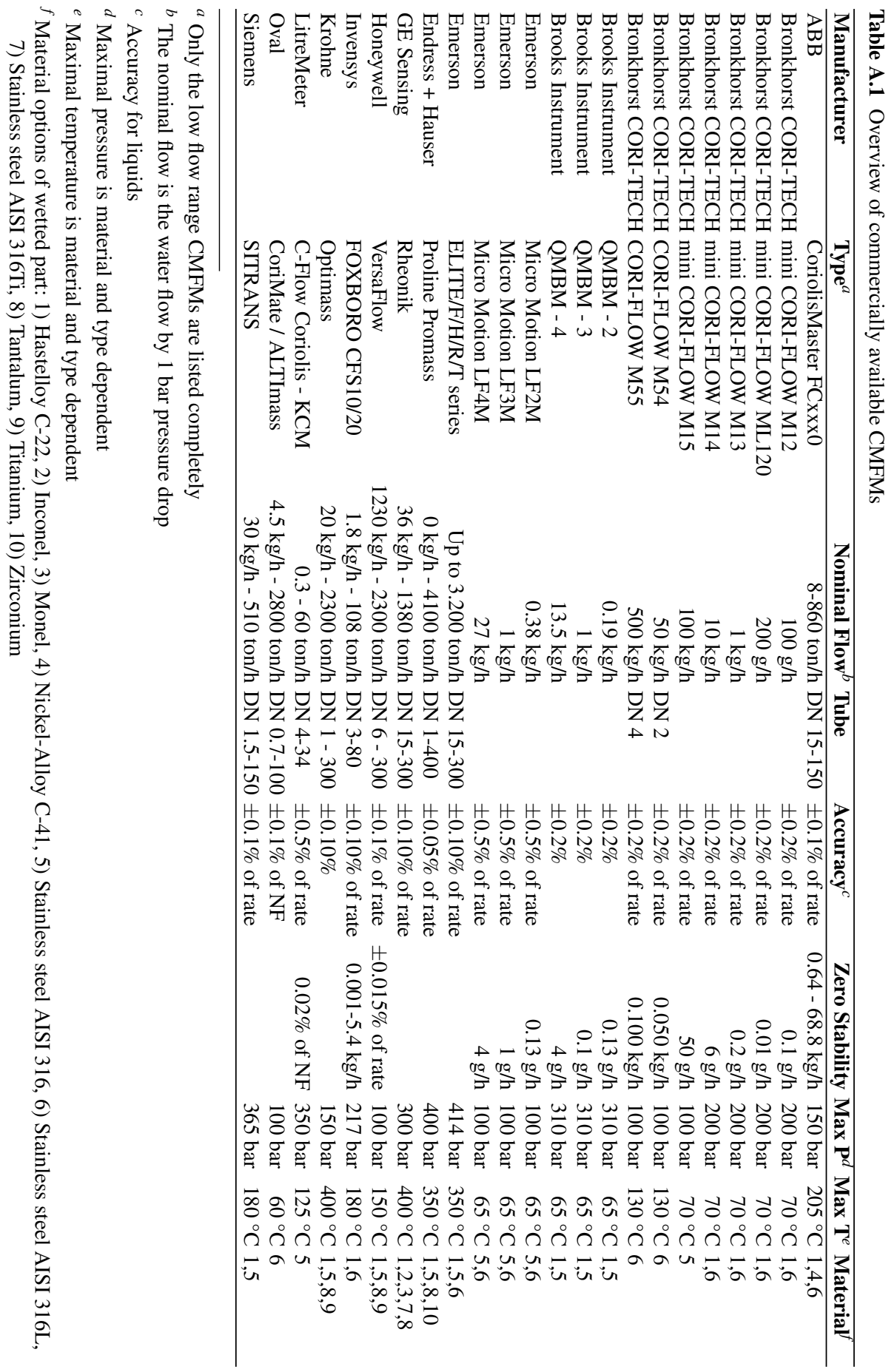




\section{Appendix B \\ 2-DOF CMFM model}

In this appendix a model of a simple CMFM with two degrees-of-freedom is presented. First, the equations of motion are derived. Using these equations, the sensitivity for flow is calculated. This model is used in Chapter 6 to determine the influence of external vibrations when scaling the nominal flow range of a CMFM.

\section{B.1 Model}

In Chapter 2 a full 3D model of a CMFM is presented. In this appendix a reduced model of a CMFM is set up, to be able to derive analytical equations [67]. This simple model has two degrees-of-freedom, which are representing the Coriolis and actuation mode only. Using a modal reduction technique, the 3D model can be transformed to the 2-DOF model. The model (Fig. B.1) contains a rigid tube filled with a moving fluid. The tube is flexibly mounted to the surroundings and the tube-movements are measured by two sensors. Note that stiffness

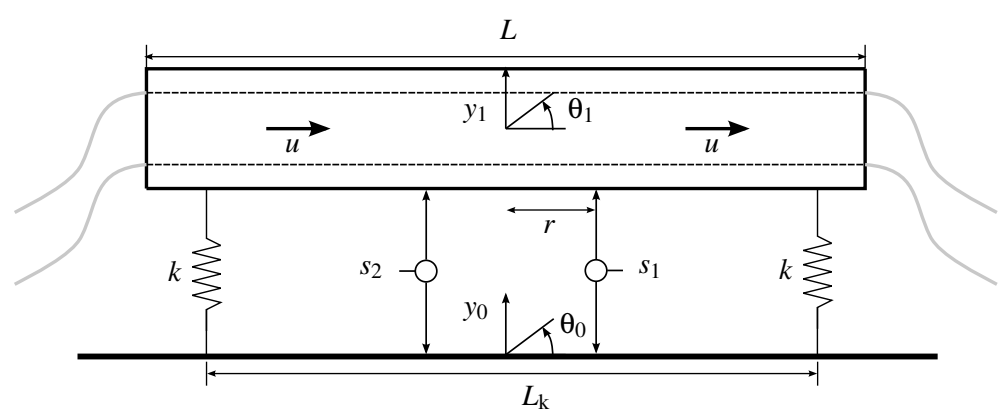

Fig. B.1 2-DOF CMFM model: a rigid fluid-conveying tube of length $L$, flexibly mounted to the surroundings by stiffness $k$. The tube-movements are measured by two sensors, placed on equal distance $r$ of the rotation axis.

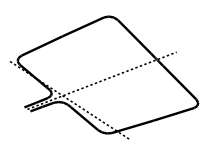


is mainly the effect of the connection tubes. The fluid is modelled as a frictionless plug flow. In this section the equations of motion are derived, first for the fluid and secondly for the tube including the fluid.

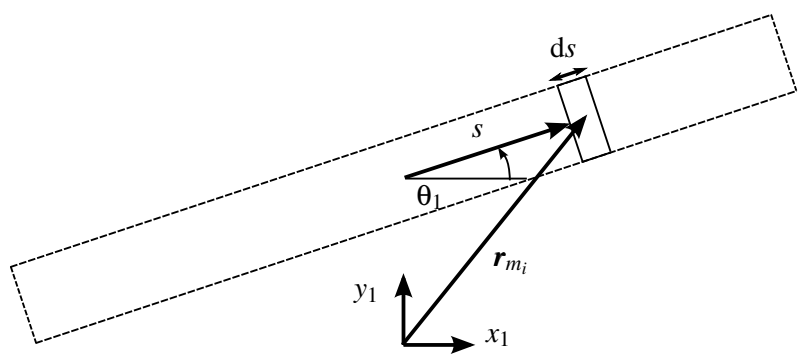

Fig. B.2 Location of one fluid element

\section{B.1.1 Fluid}

First, the forces and moments on the fluid are derived. The fluid is modelled using a series of moving fluid elements with a cross sectional area $A_{\mathrm{f}}=\frac{1}{4} \pi d^{2}$, density $\rho_{\mathrm{f}}$ and lenght $\mathrm{d} s$. In Fig. B.2, a fluid element is depicted. The mass of each element is equal to:

$$
m_{i}=\rho_{\mathrm{f}} A_{\mathrm{f}} \mathrm{d} s
$$

The position of a fluid element is equal to:

$$
\boldsymbol{r}_{m_{i}}=\left[\begin{array}{c}
s \cos \left(\theta_{1}\right) \\
y_{1}+s \sin \left(\theta_{1}\right) \\
0
\end{array}\right] \approx\left[\begin{array}{c}
s \\
y_{1}+s \theta_{1} \\
0
\end{array}\right]
$$

where a small angle approximation is used. The velocity of a fluid element is equal to:

$$
\dot{\boldsymbol{r}}_{m_{i}}=\left[\begin{array}{c}
\dot{s} \cos \left(\theta_{1}\right)-s \dot{\theta}_{1} \sin \left(\theta_{1}\right) \\
\dot{y}_{1}+\dot{s} \sin \left(\theta_{1}\right)+s \dot{\theta}_{1} \cos \left(\theta_{1}\right) \\
0
\end{array}\right] \approx\left[\begin{array}{c}
\dot{s}-s \dot{\theta}_{1} \theta_{1} \\
\dot{y}_{1}+\dot{s} \theta_{1}+s \dot{\theta}_{1} \\
0
\end{array}\right]
$$

Total linear momentum of the fluid is equal to:

$$
\boldsymbol{p}=\Sigma m_{i} \cdot \dot{\boldsymbol{r}}_{m_{i}}=\int_{-\frac{1}{2} L}^{\frac{1}{2} L}\left[\begin{array}{c}
\dot{s}-s \dot{\theta}_{1} \theta_{1} \\
\dot{y}_{1}+\dot{s} \theta_{1}+s \dot{\theta}_{1} \\
0
\end{array}\right] \rho_{\mathrm{f}} A_{\mathrm{f}} \mathrm{d} s=\rho_{\mathrm{f}} A_{\mathrm{f}} L\left[\begin{array}{c}
\dot{s} \\
\dot{y}_{1}+\dot{s} \theta_{1} \\
0
\end{array}\right]
$$


Using Newton's second law, the total net forces on the fluid are determined:

$$
\Sigma \boldsymbol{F}=\dot{\boldsymbol{p}}=\rho_{\mathrm{f}} A_{\mathrm{f}} L\left[\begin{array}{c}
\ddot{s} \\
\ddot{y}_{1}+\ddot{s} \theta_{1}+\dot{s} \dot{\theta}_{1} \\
0
\end{array}\right]
$$

The total angular momentum of the fluid is equal to:

$$
\begin{aligned}
& \boldsymbol{H}=\Sigma \boldsymbol{r}_{m_{i}} \times m_{i} \cdot \dot{\boldsymbol{r}}_{m_{i}}=\int_{-\frac{1}{2} L}^{\frac{1}{2} L}\left|\begin{array}{ccc}
i & j & k \\
s & \left(y_{1}+s \theta_{1}\right) & 0 \\
\left(\dot{s}-s \dot{\theta}_{1} \theta_{1}\right) & \left(\dot{y}_{1}+\dot{s} \theta_{1}+s \dot{\theta}_{1}\right) & 0
\end{array}\right| \rho_{\mathrm{f}} A_{\mathrm{f}} \mathrm{d} s \\
& =\int_{-\frac{1}{2} L}^{\frac{1}{2} L}\left[\begin{array}{c}
0 \\
0 \\
s\left(\dot{y}_{1}+\dot{s} \theta_{1}+s \dot{\theta}_{1}\right)-\left(y_{1}+s \theta_{1}\right)\left(\dot{s}-s \dot{\theta}_{1} \theta_{1}\right)
\end{array}\right] \rho_{\mathrm{f}} A_{\mathrm{f}} \mathrm{d} s \\
& =\rho_{\mathrm{f}} A_{\mathrm{f}}\left[\begin{array}{c}
0 \\
0 \\
\frac{1}{12} L^{3} \dot{\theta}_{1}+\frac{1}{12} L^{3} \dot{\theta}_{1} \theta_{1}^{2}-L \dot{s} y_{1}
\end{array}\right] \approx \rho_{\mathrm{f}} A_{\mathrm{f}}\left[\begin{array}{c}
0 \\
0 \\
\frac{1}{12} L^{3} \dot{\theta}_{1}-L \dot{s} y_{1}
\end{array}\right]
\end{aligned}
$$

where the quadratic term is neglected, because of the assumption of small motions. Using Newton's second law, the total net moments on the fluid are determined:

$$
\Sigma \boldsymbol{M}=\dot{\boldsymbol{H}}=\rho_{\mathrm{f}} A_{\mathrm{f}}\left[\begin{array}{c}
0 \\
0 \\
\frac{1}{12} L^{3} \ddot{\theta}_{1}-L \ddot{s} y_{1}-L \dot{s} \dot{y}_{1}
\end{array}\right]
$$

In Eq. B.5 and B.7 the mass-flow can be expressed as:

$$
\dot{\Phi}_{\mathrm{m}}=\rho_{\mathrm{f}} A_{\mathrm{f}} u
$$

where $u=\dot{s}$ is the velocity of the fluid. Since the fluid is contained in the tube, the tube exerts forces on the fluid to make it trace the tube's motion. Vice versa, the fluid exerts forces on the tube, depending the tube's motion, which are therefore termed the flow induced forces. For the 2-DOF system, we have the following flow induced force and moment, based on Eq. B.5 and B.7.

$$
\left[\begin{array}{c}
F_{y, \text { fluid }} \\
M_{\theta, \text { fluid }}
\end{array}\right]=\left[\begin{array}{cc}
\rho_{\mathrm{f}} A_{\mathrm{f}} L & 0 \\
0 & \frac{\rho_{\mathrm{f}} A_{\mathrm{f}} L^{3}}{12}
\end{array}\right]\left[\begin{array}{c}
\ddot{y}_{1} \\
\ddot{\theta}_{1}
\end{array}\right]+\left[\begin{array}{cc}
0 & \dot{\Phi}_{\mathrm{m}} L \\
-\dot{\Phi}_{\mathrm{m}} L & 0
\end{array}\right]\left[\begin{array}{c}
\dot{y}_{1} \\
\dot{\theta}_{1}
\end{array}\right]+\left[\begin{array}{cc}
0 & \rho_{\mathrm{f}} A_{\mathrm{f}} L \dot{u} \\
-\rho_{\mathrm{f}} A_{\mathrm{f}} L \dot{u} & 0
\end{array}\right]\left[\begin{array}{l}
y_{1} \\
\theta_{1}
\end{array}\right]
$$




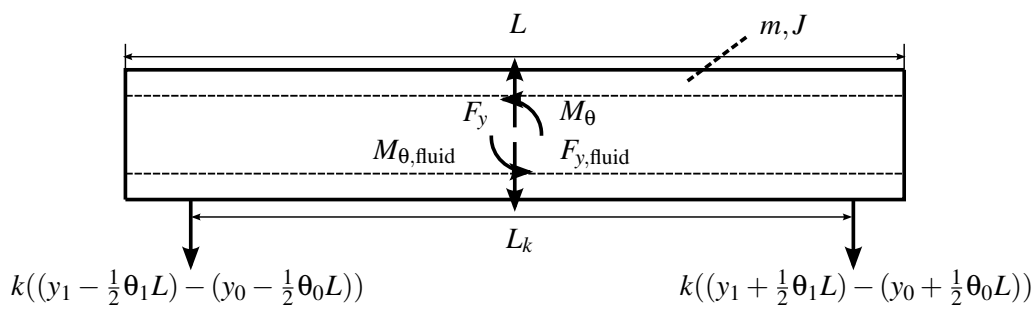

Fig. B.3 Free body diagram of the tube, where $F_{y, \text { fluid }}$ and $M_{\theta, \text { fluid }}$ are the flow induced force and moment. Further, on the tube an external force $F_{y}$ and moment $M_{\theta}$ are applied.

\section{B.1.2 Tube}

Second, the equations of motion of the tube, including the fluid flow, are derived. The free body diagram of the tube is depicted in Fig. B.3, where $m$ and $J=\frac{1}{12} m L^{2}$ are respectively the mass and moment of inertia of the empty, rigid tube. The tube has a length $L$ and is suspended by stiffness $k$ and $L_{k}$ is the ratio between the translational and rotational stiffness. The structural damping is omitted in this model, because the Coriolis tubes are normally designed with high Q-factors [44]. Using Newton's second law, the equations of motion are derived as:

$$
\begin{aligned}
\Sigma F=m \ddot{y}_{1} & =-k\left(y_{1}-y_{0}\right)-k\left(y_{1}-y_{0}\right)+F_{y}-F_{y, \text { fluid }} \\
& =-2 k y_{1}+2 k y_{0}+F_{y}-F_{y, \text { fluid }} \\
\Sigma M=J \ddot{\theta}_{1} & =\frac{1}{2} L_{k} k\left(-\frac{1}{2} \theta_{1} L_{k}+\frac{1}{2} \theta_{0} L_{k}\right)-\frac{1}{2} L_{k} k\left(\frac{1}{2} \theta_{1} L_{k}-\frac{1}{2} \theta_{0} L_{k}\right)+M_{\theta}-M_{\theta, \text { fluid }} \\
& =-\frac{1}{2} \theta_{1} L_{k}^{2} k+\frac{1}{2} \theta_{0} L_{k}^{2} k+M_{\theta}-M_{\theta, \text { fluid }}
\end{aligned}
$$

where $F_{y \text {,fluid }}$ and $M_{\theta \text {,fluid }}$ are the flow induced force and moment, see Eq. B.9. Further, on the tube an external force $F_{y}$ and moment $M_{\theta}$ can be applied. Using Eq. B.9. B.10 and B.11, the equations of motion of the fluid, the tube and its suspension can be written in matrix vector notation:

$$
\begin{aligned}
{\left[\begin{array}{cc}
m+\rho_{\mathrm{f}} A_{\mathrm{f}} L & 0 \\
0 & J+\frac{1}{12} \rho_{\mathrm{f}} A_{\mathrm{f}} L^{3}
\end{array}\right] } & {\left[\begin{array}{l}
\ddot{y}_{1} \\
\ddot{\theta}_{1}
\end{array}\right]+\left[\begin{array}{cc}
0 & \dot{\Phi}_{\mathrm{m}} L \\
-\dot{\Phi}_{\mathrm{m}} L & 0
\end{array}\right]\left[\begin{array}{l}
\dot{y}_{1} \\
\dot{\theta}_{1}
\end{array}\right] } \\
& +\left[\begin{array}{cc}
2 k & \rho_{\mathrm{f}} A_{\mathrm{f}} L \dot{u} \\
-\rho_{\mathrm{f}} A_{\mathrm{f}} L \dot{u} & \frac{1}{2} k L_{k}^{2}
\end{array}\right]\left[\begin{array}{c}
y_{1} \\
\theta_{1}
\end{array}\right]=\left[\begin{array}{c}
F_{y}+2 k y_{0} \\
M_{\theta}+\frac{1}{2} k L_{k}^{2} \theta_{0}
\end{array}\right]
\end{aligned}
$$

For the no-flow condition $(u=0)$, the tube has two clear resonance frequencies:

$$
\omega_{1}=\sqrt{\frac{2 k}{m+\rho_{\mathrm{f}} A_{\mathrm{f}} L}}, \quad \omega_{2}=\sqrt{\frac{\frac{1}{2} k L_{k}^{2}}{J+\frac{1}{12} \rho_{\mathrm{f}} A_{\mathrm{f}} L^{3}}}=\frac{\sqrt{3} L_{k}}{L} \sqrt{\frac{2 k}{m+\rho_{\mathrm{f}} A_{\mathrm{f}} L}}=\alpha \omega_{1},
$$


belonging respectively to the translational and rotational mode of the tube. The resonance frequencies depend on the geometry, but also on the density $\rho_{\mathrm{f}}$ of the fluid.

To measure the motion of the tube, two relative-motion sensors are added to the model, located at equal distance $r$ from the rotation axis of actuation:

$$
\left[\begin{array}{l}
s_{1} \\
s_{2}
\end{array}\right]=\left[\begin{array}{cccc}
-1 & 1 & -r & r \\
-1 & 1 & r & -r
\end{array}\right]\left[\begin{array}{llll}
y_{0} & y_{1} & \theta_{0} & \theta_{1}
\end{array}\right]^{T}
$$

\section{B.2 Phase difference}

Before the sensitivity for flow is calculated, it is explained how the measurement value is obtained, using the phase of the sensor signals. The fluid conveying tube is actuated to oscillate in resonance with a low amplitude. A fluid flow in the vibrating tube induces Coriolis forces, proportional to the mass-flow, which are affecting the modeshape of the actuation mode. This effect is modelled by the coupling terms in the damping matrix (see Eq. B.12). The motion due to the Coriolis effect is $90^{\circ}$ out-of-phase with the actuation displacement, because the Coriolis-terms are velocity dependent, but both motions are sinusoidal with the same angular frequency. Thus, without external vibrations, the sensor signal $s_{1}$ is equal to:

$$
s_{1}=y_{\mathrm{act}} \sin \left(\omega_{\mathrm{act}} t\right)+y_{\mathrm{cor}} \cos \left(\omega_{\mathrm{act}} t\right)=A_{1} \sin \left(\omega_{\mathrm{act}} t+\phi_{1}\right)
$$

where $y_{\text {act }}$ is the actuation displacement and $y_{\text {cor }}$ the flow induced motion. The amplitude and phase of the sensor signal can be related to the actuation and Coriolis displacement, by extending Eq. B.15

$$
A_{1} \sin \left(\omega_{\text {act }} t+\phi_{1}\right)=A_{1}\left(\cos \left(\phi_{1}\right) \sin \left(\omega_{\text {act }} t\right)+\sin \left(\phi_{1}\right) \cos \left(\omega_{\text {act }} t\right)\right)
$$

The actuation and Coriolis displacement amplitude are thus equal to:

$$
\begin{aligned}
& y_{\mathrm{act}}=A_{1} \cos \left(\phi_{1}\right) \\
& y_{\mathrm{cor}}=A_{1} \sin \left(\phi_{1}\right)
\end{aligned}
$$

The phase of the sensor signal, which is independent of the amplitude $A_{1}$, is calculated as follows:

$$
\frac{A_{1} \sin \left(\phi_{1}\right)}{A_{1} \cos \left(\phi_{1}\right)}=\frac{y_{\text {cor }}}{y_{\text {act }}}=\tan \phi_{1} \approx \phi_{1}
$$

The same is done for the second sensor, resulting in $\phi_{2}$. The sensors are placed at opposite sides of the rotation axis, therefore the result is the opposite. This results in a phase difference between the two sensor signals:

$$
\Delta \phi=\phi_{1}-\phi_{2} \approx 2 \frac{y_{\mathrm{cor}}}{y_{\mathrm{act}}}
$$


In practice, the phase of the sensor signals is measured directly, without determining the amplitudes of the sensor signals. There are different digital signal processing methods that can be applied. A method is to apply dual quadrature demodulation, for which the implementation to a CMFM is described by Mehendale [44]. A phase-locked loop algorithm is implemented to compute the resonance frequency of the oscillating tube. The low-pass filtered frequency is used to create two waveforms: a sine and a cosine. The measured sensor signal mixed with both waveforms, resulting in a signal with an offset and a double frequency component. The double frequency component is then filtered out with a low-pass filter (LPF). This calculation thus results in two DC values, depending on the phase between the sensor signals and the newly introduced waveform. Besides the removal of the second frequency, the LPF is the key in the trade-off between speed of the flow measurement and the measurement noise. The algorithm is explained in more detail in section 2.3.2.1

\section{B.3 Sensitivity for flow}

In this section, the measurement sensitivity for mass-flow of the model, depicted in Fig. B.1 is determined. The mass-flow measurement sensitivity is calculated for two cases, a translational and a rotational actuation mode. In this section, the external displacements $\left(y_{0}=\theta_{0}=\right.$ 0 ), external forces and acceleration of the fluid $\dot{u}=0$ of the model are omitted.

The tube is actuated to resonate in one of its modeshapes. The Coriolis-effect results in a $90^{\circ}$ out-of-phase actuation of the other modeshape. The actuation displacement and the induced Coriolis displacement both occur at the actuation frequency. Two sensors, on both sides of the rotation axis, measure the translation and rotation of the tube, in this model just one harmonic. In the first case, the translational mode is used as actuation mode. The second equation of Eq B.12 gives the induced effect. The Laplace transform of this equation is:

$$
\left(J+\frac{1}{12} \rho_{\mathrm{f}} A_{\mathrm{f}} L^{3}\right) s^{2} \theta_{1}-\dot{\Phi}_{\mathrm{m}} L s y_{1}+\frac{1}{2} k L_{k}^{2} \theta_{1}=0
$$

Evaluating the Laplace transform for the argument $s=i \omega_{1}$, where $\omega_{1}$ is the resonance frequency of the translational mode, results in the induced rotation:

$$
\theta_{1}=\frac{\dot{\Phi}_{\mathrm{m}} L \omega_{1} y_{1} \mathrm{i}}{\left(-\left(J+\frac{1}{12} \rho_{\mathrm{f}} A_{\mathrm{f}} L^{3}\right) \omega_{1}^{2}+\frac{1}{2} k L_{k}^{2}\right)}=\frac{\dot{\Phi}_{\mathrm{m}} L \omega_{1} y_{1} \mathrm{i}}{\left(J+\frac{1}{12} \rho_{\mathrm{f}} A_{\mathrm{f}} L^{3}\right)\left(\omega_{2}^{2}-\omega_{1}^{2}\right)}
$$

The phase difference (see Eq. B.20), using the fact that the Coriolis displacement is $90^{\circ}$ outof-phase with the actuation displacement, is determined as:

$$
\Delta \phi_{1} \approx 2 \frac{\mathfrak{I}\left(y_{\text {cor }}\right)}{\mathfrak{R}\left(y_{\text {act }}\right)}=2 \frac{\mathfrak{I}\left(s_{1}-s_{2}\right)}{\mathfrak{R}\left(s_{1}+s_{2}\right)}=2 \frac{\mathfrak{I}\left(2 r \theta_{1}\right)}{\mathfrak{R}\left(2 y_{1}\right)}=\frac{2 r L}{\left(J+\frac{1}{12} \rho_{\mathrm{f}} A_{\mathrm{f}} L^{3}\right)} \frac{\omega_{1}}{\omega_{2}^{2}-\omega_{1}^{2}} \dot{\Phi}_{\mathrm{m}}
$$

The mass-term can be written alternatively as: 


$$
J+\frac{1}{12} \rho_{\mathrm{f}} A_{\mathrm{f}} L^{3}=\frac{1}{12} m L^{2}+\frac{1}{12} \rho_{\mathrm{f}} A_{\mathrm{f}} L^{3}=\frac{L^{2}}{12}\left(m+\rho_{\mathrm{f}} A_{\mathrm{f}} L\right)
$$

Using Eq. B.13 the denominator of Eq. B.23 can be rewritten as:

$$
\left(m+\rho_{\mathrm{f}} A_{\mathrm{f}} L\right)\left(\omega_{2}^{2}-\omega_{1}^{2}\right)=\left(m+\rho_{\mathrm{f}} A_{\mathrm{f}} L\right) \omega_{1}^{2}\left(\alpha^{2}-1\right)=2 k\left(\alpha^{2}-1\right)
$$

Rewriting Eq. B.23 gives:

$$
\Delta \phi_{1} \approx \frac{24 r}{L\left(m+\rho_{\mathrm{f}} A_{\mathrm{f}} L\right)} \frac{\omega_{1}}{\omega_{1}^{2}\left(\alpha^{2}-1\right)} \dot{\Phi}_{\mathrm{m}}=\frac{24 r}{L\left(\alpha^{2}-1\right)} \frac{1}{\sqrt{2 k\left(m+\rho_{\mathrm{f}} A_{\mathrm{f}} L\right)}} \dot{\Phi}_{\mathrm{m}}
$$

The phase difference depends on the fluid density, in contrast to the following time difference:

$$
\Delta t_{1}=\frac{\Delta \phi_{1}}{\omega_{1}} \approx \frac{2 r L}{\left(J+\frac{1}{12} \rho_{\mathrm{f}} A_{\mathrm{f}} L^{3}\right)} \frac{1}{\omega_{2}^{2}-\omega_{1}^{2}} \dot{\Phi}_{\mathrm{m}}=\frac{24 r}{L} \frac{1}{2 k\left(\alpha^{2}-1\right)} \dot{\Phi}_{\mathrm{m}}
$$

Alternatively, the rotational mode can be used as actuation mode. The second equation of Eq B.12 gives the induced effect. The Laplace transform of this equation is:

$$
\left(m+\rho_{\mathrm{f}} A_{\mathrm{f}} L\right) s^{2} y_{1}+\dot{\Phi}_{\mathrm{m}} L s \theta_{1}+2 k y_{1}=0
$$

Evaluating the Laplace transform for the argument $s=i \omega_{2}$, where $\omega_{2}$ is the resonance frequency of the rotational mode, results in the induced translation:

$$
y_{1}=\frac{-\dot{\Phi}_{\mathrm{m}} L \omega_{2} \theta_{1} \mathrm{i}}{\left(-\left(m+\rho_{\mathrm{f}} A_{\mathrm{f}} L\right) \omega_{2}^{2}+2 k\right)}=\frac{\dot{\Phi}_{\mathrm{m}} L \omega_{2} \theta_{1} \mathrm{i}}{\left(m+\rho_{\mathrm{f}} A_{\mathrm{f}} L\right)\left(\omega_{2}^{2}-\omega_{1}^{2}\right)}
$$

The phase difference and time difference are determined as:

$$
\begin{aligned}
\Delta \phi_{2} & \approx 2 \frac{\mathfrak{I}\left(y_{\text {cor }}\right)}{\mathfrak{R}\left(y_{\text {act }}\right)}=2 \frac{\mathfrak{I}\left(s_{1}+s_{2}\right)}{\mathfrak{R}\left(s_{1}-s_{2}\right)}=2 \frac{\mathfrak{I}\left(2 y_{1}\right)}{\mathfrak{R}\left(2 r \theta_{1}\right)}=\frac{2 L}{r\left(m+\rho_{\mathrm{f}} A_{\mathrm{f}} L\right)} \frac{\omega_{2}}{\omega_{2}^{2}-\omega_{1}^{2}} \dot{\Phi}_{\mathrm{m}} \\
& =\frac{2 L \alpha \omega_{1}}{r\left(m+\rho_{\mathrm{f}} A_{\mathrm{f}} L\right) \omega_{1}^{2}\left(\alpha^{2}-1\right)} \dot{\Phi}_{\mathrm{m}}=\frac{2 L \alpha}{r\left(\alpha^{2}-1\right)} \frac{1}{\sqrt{2 k\left(m+\rho_{\mathrm{f}} A_{\mathrm{f}} L\right)}} \dot{\Phi}_{\mathrm{m}} \\
\Delta t_{2} & =\frac{\Delta \phi_{2}}{\omega_{2}} \approx \frac{2 L}{r\left(m+\rho_{\mathrm{f}} A_{\mathrm{f}} L\right)} \frac{1}{\omega_{2}^{2}-\omega_{1}^{2}} \dot{\Phi}_{\mathrm{m}}=\frac{2 L}{r} \frac{1}{2 k\left(\alpha^{2}-1\right)} \dot{\Phi}_{\mathrm{m}}
\end{aligned}
$$

The the actual measurement is the time-difference, which is a function of the mass-flow $\dot{\Phi}_{\mathrm{m}}$. The measurement sensitivity for flow is defined as:

$$
S_{i}=\frac{\Delta t_{i}}{\dot{\Phi}_{\mathrm{m}}}
$$

The sensitivity depends on the sensor position ratio $\frac{r}{L}$, the tube stiffness $k$ and the frequency separation factor $\alpha$. Note that the sensitivity for flow is not a constant in real CMFMs, e.g. the stiffness depends on the temperature. 


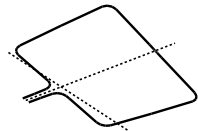




\title{
Appendix C \\ Patent: CMFM with active vibration isolation control
}

\begin{abstract}
The invention relates to a Coriolis flow sensor. The sensor comprises a housing and at least a Coriolis-tube with at least two ends being fixed in a tube fixation means. The flow sensor comprises excitation means for causing the tube to oscillate, as well as detection means for detecting at least a measure of displacements of parts of the tube during operation. According to the invention, the Coriolis flow sensor comprises a reference mass, as well as further excitation means arranged for causing the reference mass to oscillate during operation, as well as further detection means for detecting at least a measure of displacements of the reference mass during operation. Additionally, control means are provided for controlling the excitation means and/or further excitation means based on vibrations measured by the detection means and/or further detection means. This way a Coriolis flow sensor with active vibration isolation is obtained.
\end{abstract}

This chapter is reprinted from: J. C. Lötters, L. van de Ridder, W. D. Kruijswijk, W. B. J. Hakvoort, and M. R. Katerberg. Coriolis flow meter with active vibration isolation, Patent pending, 25 November 2013. 


\section{C.1 Description}

The invention relates to a Coriolis flow sensor, comprising at least a Coriolis-tube with at least two ends being fixed in a tube fixation means, wherein the flow sensor comprises excitation means for causing the tube to oscillate, as well as detection means for detecting at least a measure of displacements of parts of the tube during operation.

A Coriolis flow sensor having a loop-shaped Coriolis tube is known from EP1719982A1 [45]. Various types of loop-shaped Coriolis tubes are described therein, both of the single loop type and of the (continuous) double loop type. The present invention relates to any of these types, but is not restricted thereto.

A Coriolis flow sensor (also indicated as flow sensor of the Coriolis type) comprises at least one vibrating tube, often denoted Coriolis tube, flow tube, or sensing tube. This tube or these tubes is or are fastened at both ends to the housing of the instrument. These tube ends serve at the same time as feed and discharge ducts for the liquid or gas flow to be measured.

Besides the flow tube (or tubes), a Coriolis flow sensor comprises two further subsystems, i.e. one for excitation and one for detection. The excitation system (exciter) is arranged for bringing the tube into vibration. For this purpose, one or several forces or torques are applied to portions of the tube. The detection system is arranged for detecting at least a measure of the displacements of one or several points of the tube as a function of time. Instead of this displacement, the force (or torque) exerted by the tube on its environment may alternatively be measured. The same holds true for the velocity, acceleration and strain of the tube; what will be described below with reference to displacement detection is equally valid for force detection, velocity detection, acceleration detection and/or strain detection.

As a fluid flows in the vibrating tube, it induces Coriolis forces, proportional to the massflow, which affect the tube motion and change the mode shape. Measuring the tube displacement using the detection system, the change of the mode shape may be measured, which allows for mass-flow measurements.

The vibration of the tube generated by the exciter takes place at a more or less fixed frequency which varies slightly as a function, amongst others, of the density of the medium flowing through the tube. The vibration frequency is almost always a natural frequency of the tube so that a maximum amplitude can be achieved with a minimum energy input.

Besides the effect of the mass-flow on the mode shape of the tube, external vibrations can introduce motions that cannot be distinguished from the Coriolis force induced motion. The external vibrations create additional components in the Coriolis flow sensor signals, and those additional components can introduce a measurement error. For low flows, the Coriolis force induced motion is relatively small compared to external vibrations induced motions. Coriolis flow sensors designed to be sensitive to low flows, are normally rather sensitive to external vibrations.

To minimize the influence of floor vibration on the measurement value, it is known in the prior art to use so called passive vibration isolation. According to the prior art, passive isolation consist of several stages of mass-spring damper systems between the floor and the tube fixation means, the parameters are adjusted to achieve high-frequency attenuation, which is appropriate for many applications. The better the vibration isolation system the better the decoupling of the internal measurement system from any environmental disturbances. 
It is an object of the present invention to provide an improved Coriolis flow sensor, in particular a Coriolis flow sensor designed to be sensitive to low flows, wherein the influence of external vibrations is reduced.

To this end, the invention provides a Coriolis flow sensor, that is characterized in that it comprises a reference mass, further excitation means arranged for causing the reference mass to oscillate, as well as further detection means. The further detection means are in an embodiment arranged for detecting at least a measure of displacements of the reference mass during operation. With this, it is meant that the further detection means are at least arranged for detecting relative displacements of the reference mass, for instance between the reference mass and the Coriolis tube, or between the reference mass and the housing. In this sense, it is thinkable that the further detection means are arranged for detecting at least a measure of displacements of the housing during operation. A detection of displacements of both the reference mass and the housing is also possible. An absolute displacement detection (of the reference mass and/or the housing) is conceivable as well. Additionally, control means are provided for controlling the

excitation means and/or further excitation means based on movements and/or vibrations measured by the detection means and/or further detection means.

The reference mass together with the further excitation means and the further detection means provide in effect active vibration isolation means which are arranged for active vibration isolation, in order to minimize the effect external vibrations have on the Coriolis-tube. This may in general be done by means of two principles, or a combination thereof, which two principles will be elucidated below.

Using the first principle, the Coriolis-tube, the reference mass, and the housing may be connected in series. The reference mass is then connected to the Coriolis-tube, in particular to the tube fixation means. The further excitation means may be used to counter movements of the housing so as to substantially fix the absolute position of the reference mass, or in other words to create a stable ground for the Coriolis-tube, such that vibrations or other disturbances on the housing do not result in vibrations on the Coriolis-tube, and hence do not affect the Coriolis force induced vibrations on the Coriolis-tube.

Using the second principle, the Coriolis-tube and the reference mass are positioned in parallel to each other, each being connected to the housing. The housing is then connected to the Coriolis-tube, in particular to the tube fixation means. The further excitation means and further detection means may be used to match the dynamic properties of the reference mass with the dynamic properties of the Coriolis-tube in response to external vibrations. Thus, disturbances due to external vibrations acting on the housing act on both the reference mass and the Coriolis-tube, in a similar manner. Thereby, the relative displacements of the Coriolis tube are at least substantially insensitive to external vibration, but still sensitive to the Coriolis force. Alternatively, disturbances on the reference mass, that are (directly or indirectly) measured by the further detection means may be used to correct the measurements obtained by the detection means for detecting at least a measure for displacements of the Coriolis-tube.

A combination of the first and second principle is thinkable, which uses the set-up of the first principle with an additional reference mass connected to the reference mass, positioned in parallel to the Coriolis-tube. The first reference mass is used as a stable ground for the 
Coriolis-tube, and the additional reference mass may be used to filter out any further disturbances that still act on the additional reference mass and the Coriolis tube.

Thus, it is shown that the Coriolis flowsensor according to the present invention is less sensitive to external vibrations, and hence the object of the current invention is achieved.

Advantageous embodiments of the present invention are subject of the dependent claims, and will be elucidated below.

In an embodiment, the reference mass is connected to the housing. This provides for a relatively compact embodiment of the flow sensor, since it allows the components of the active vibration isolation to be positioned within a casing of the flow sensor.

The reference mass may be connected to the housing by means of spring elements, in particular flexures or spring-leafs. This provides for an effective, yet relatively compact and cheap construction.

According to an aspect, active vibration isolation is accomplished by means of a first principle, which substantially fixes the position of the reference mass. The reference mass may comprise the tube fixation means, or at least the tube fixation means are substantially rigidly connected to the reference mass. The control means are arranged for moving the reference mass in opposite directions to movements of the housing, in particular movements due to external vibrations acting on the housing.

The further excitation means are in an embodiment provided between the housing or a part rigidly connected to the housing, on the one hand, and to the reference mass on the other hand.

The further excitation means may comprise a number of excitation members. For instance, a single excitation member, such as a Piezo element and/or Lorentz actuator, may suffice to eliminate some vibrations acting on the housing. In line with this, a single detection member may suffice to detect the displacement of the reference mass.

According to an improved embodiment, the excitation means comprise at least three excitation members. In addition, it is conceivable that the detection means comprise at least three detection members.

The further detection means are in an embodiment arranged for detecting at least a measure for movements of the housing or a part rigidly connected thereto. The control means are in an embodiment arranged for moving the reference mass with respect to the housing based on feedforward control. In an embodiment, the control means are arranged for oscillating the reference mass based on feedforward control. Moving or oscillating preferably counters movements of the housing, in particular due to external vibrations.

The further detection means are in an embodiment arranged for detecting at least a measure for movements of the reference mass. The control means are arranged for moving, for instance oscillating, the reference mass with respect to the housing, based on feedback control.

The further detection means are in an embodiment arranged for detecting at least a measure for movements of the housing, and additional detection means are provided for detecting at least a measure for movements of the reference mass, and wherein the control means are arranged for moving, for instance oscillating, the reference mass with respect to the housing based on both feedback control and feedforward control.

The reference mass may comprise the excitation means for the Coriolis tube. 
The reference mass may comprise the detection means for the Coriolis tube.

According to an aspect, active vibration isolation is accomplished by means of a second principle, which substantially matches the dynamic properties of the reference mass to the dynamic properties of the Coriolis tube in response to external vibrations. The housing may comprise the tube fixation means, or at least the tube fixation means is rigidly connected to the housing. The control means are arranged for moving the reference mass substantially identically to the Coriolis tube in response to movements of the housing, in particular movements due to external vibrations acting on the housing. Thereby the relative motion of the Coriolis tube and reference mass is not affected by movements of the housing. The relative motion may be measured directly or reconstructed from the measured motion of the Coriolis tube and reference mass. A feedback control scheme, a feedforward control scheme, or a combination of these may be used.

According to an aspect, active vibration isolation is accomplished by means of the first and second principle described above. In this sense, an additional reference mass is used, in combination with the reference mass.

In an embodiment, the flow sensor comprises an additional reference mass which may be connected to the reference mass, additional excitation means arranged for causing the additional reference mass to oscillate, as well as additional detection means for detecting at least a measure for displacements of the additional reference mass during operation. Additionally, control means are provided for controlling the excitation means and/or further excitation means and/or additional excitation means based on vibrations measured by the detection means and/or further detection means and/or additional detection means.

The detection means (or further detection means, or additional detection means) may be arranged for detecting at least a measure for displacements of a body (such as the housing, the reference mass, additional reference mass, and/or the Coriolis tube). In general it can be said that the detection means are not limited thereto, but that acceleration, velocity, position, strain and/or force of that specific part may be measured as well.

The excitation means (or further excitation means, or additional excitation means) may comprise a Lorentz actuator, such as a Voicecoil motor element, and/or a Piezo element.

According to an aspect, the invention provides a method for actively isolating vibrations in a flowsensor of the Coriolis type, such as the flow sensor described above, wherein the flow sensor comprises a Coriolis flow tube and a reference mass that is connected thereto in a flexible manner, wherein the method comprises the step of substantially fixing the position of the reference mass.

According to an aspect, the invention provides a method for actively isolating vibrations in a flowsensor of the Coriolis type, such as the flow sensor described above, wherein the flow sensor comprises a Coriolis flow tube and a reference mass that is movable independently thereof, wherein the method comprises the step of substantially matching the dynamical behavior of the reference mass to the dynamical behavior of the Coriolis tube.

According to an aspect, the active vibration isolation according to the invention may be accomplished together with so-called passive vibration isolation, which is known per se to those skilled in the art. The invention is not limited to the use of active vibration isolation alone.

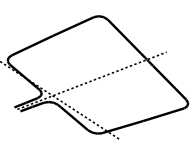


According to an aspect, the passive vibration isolation for a Coriolis flow sensor may be improved. This is, according to this aspect of the invention, obtained by a Coriolis flow sensor, comprising at least a Coriolis-tube with at least two ends being fixed in a tube fixation means, wherein the flow sensor comprises excitation means for causing the tube to oscillate, as well as detection means for detecting at least a measure of displacements of parts of the tube during operation. The Coriolis flow sensor comprises a compliant connection between the casing and at least part of the detection means. The compliant connection according to this embodiment of the invention is constructed and arranged such that a relative displacement measurement of the tube, dependent on the tube actuation and mass flow, though independent of casing excitations is obtained. It is noted that this Coriolis flow sensor, having the improved passive vibration isolation described herein, already provides improved results. The Coriolis flow sensor according to the invention may be embodied with either one of passive vibration isolation, or active vibration isolation, and/or both.

The flow sensor according to this aspect of the invention is in an embodiment arranged for causing the detection means to oscillate in response to excitations of the housing, in substantially the same manner as the Coriolis-tube oscillates in response to excitations of the housing.

This may in an embodiment be obtained by tuning the resonance frequency and/or the damping of the compliant detection means. Tuning may be dependent on the actuation frequency of the Coriolis tube, and the first and second Coriolis mode.

The tuning may comprise that the resonance frequency and/or the damping ratio are substantially equal for the Coriolis-tube (i.e. internal mode of the tube-window) and the compliant mounted detection means.

The tuning may in practice comprise that the resonance frequency and/or the damping ratio are lower for the internal mode of the tube-window (Coriolis-tube), compared to the resonance frequency and the damping ratio of the compliant mounted detection means.

The tuning may in an embodiment comprise that the resonance frequency and/or the damping ratio for the internal mode of the tube-window (Coriolis-tube) are approximately $90 \%$ of the resonance frequency and the damping ratio of the compliant mounted detection means.

It is conceivable in an alternative embodiment that the resonance frequency and the damping ratio are higher for the internal mode of the tube-window (Coriolis-tube), compared to the resonance frequency and the damping ratio of the compliant mounted detection means.

In addition to the passive vibration isolation described above, active vibration isolation may be used, in particular as described herein. Thus, the active vibration isolation may be accomplished together with the passive vibration isolation.

The invention will be described below by means of the accompanying Figures, which show several embodiments of the invention.

In the figures, it is shown:

Fig. 1a-c a perspective view, a side view and a dynamical overview of a first embodiment of the flowmeter according to the invention;

Fig. 2a-c a perspective view, a side view and a dynamical overview of a second embodiment of the flowmeter according to the invention;

Fig. 3 a dynamical overview of a third embodiment of the flowmeter according to the invention. 
Fig. 4a-c a perspective view, a side view and a dynamical overview of a third embodiment of the flowmeter according to the invention;

Fig. 5 an embodiment of a compliant sensor design for passive vibration isolation according to an aspect of the invention.

Fig. C.1(a) shows a perspective view of a flowmeter 1 of the Coriolis type (or Coriolis Mass Flow Meter, CMFM), and Fig.C.1(b) shows a side view of said flowmeter 1, according to a first embodiment of the invention. The flowmeter 1 of the Coriolis type has a looped tube 2 that is bent into a rectangular shape so as to follow a substantially circumferential path (substantially one full turn), and that comprises a flexible inlet tube 3 and a flexible outlet tube 4 for a fluid medium. Preferably, the loop 2 and the inlet and outlet tubes 3, 4 are portions of one and the same tube. The tube 2 in its entirety is bent into a rectangular shape, but the corners are rounded such that it can be bent into this shape. The inlet tube 3 is connected to a supply line 6 and the outlet tube 4 to a discharge line 7 via a supply/discharge block 5a, which forms part of a housing 5 or casing 5 . The inlet and outlet tubes 3, 4 of this embodiment extend within the loop 2 and are fastened (i.e. clamped) to a frame 15 by fastening means 12 . The fastening is provided in a location such that the free path length of the inlet and the outlet tube 3, 4 (i.e. the portion of the inlet/outlet tube 3, 4 between the connection of the second transverse tube portions $2 \mathrm{a}, 2 \mathrm{~b}$ and the location of the clamping to the fastening means 12 ) is at least $50 \%$, preferably $60 \%$ of the length of each of the lateral tube portions $2 \mathrm{c}, 2 \mathrm{~d}$, or even longer. The flexible inlet and outlet tubes 3, 4 do not form part of the loop 2 but provide a flexible fastening of the loop 2 to the frame 15. The loop 2 may thus be regarded as being flexibly suspended by means of the inlet and outlet tubes. The loop 2 and the inlet and outlet tubes 3, 4 may advantageously be manufactured from one integral piece of tubing. This may be, for example, a stainless steel tube with an outer diameter of approximately $0.7 \mathrm{~mm}$ and a wall thickness of approximately $0.1 \mathrm{~mm}$. Depending on the outer dimensions of the loop 2 and the pressure the tube is to be able to withstand (for example 100 bar), the outer diameter of the tube will usually be smaller than $1 \mathrm{~mm}$ and the wall thickness $0.2 \mathrm{~mm}$ or less. It is noted that other dimensions may be used as well.

The tube 2 consists of a substantially rectangular framework comprising two parallel lateral tubes $2 \mathrm{~d}$ and $2 \mathrm{e}$, a first transverse tube $2 \mathrm{c}$ connected to first (lower) ends of the lateral tubes $2 \mathrm{~d}$ and $2 \mathrm{e}$, and two second transverse tubes $2 \mathrm{a}$ and $2 \mathrm{~b}$ connected at one side to second (upper) ends of the lateral tubes and at the other side to the centrally returning inlet and outlet tubes 3 and 4, respectively. The rectangular loop 2 preferably has rounded corners. The tubes 3 and 4, which run closely together on either side of and symmetrically with respect to the main axis of symmetry $\mathrm{S}$ of the loop 2 , are fastened to the fastening means 12 , for example by clamping or soldering or welding, said means 12 in their turn being fastened to the frame 15, which forms a reference mass 15 as will be explained below. The inlet and outlet tubes 3 , 4 are flexible and act as it were as a suspension spring for the loop 2. This suspension allows a motion of the loop 2 both about the main axis of symmetry $S$ and about a second axis $S$ ' situated in the plane of the loop 2 and perpendicular to the main axis of symmetry $\mathrm{S}$.

To close the loop 2 mechanically (i.e. to interconnect the beginning and end of the loop mechanically, directly or indirectly), the tubes 3,4 are preferably connected to one another along the extent of their free path lengths, for example in that they are welded or soldered together.

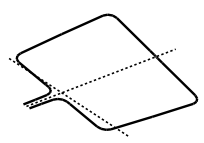




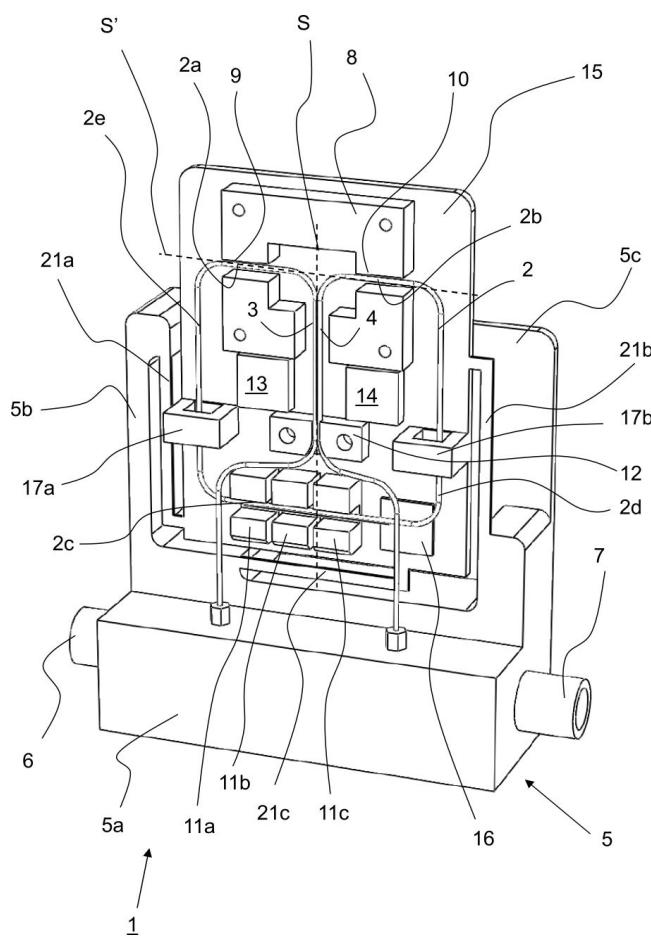

(a)

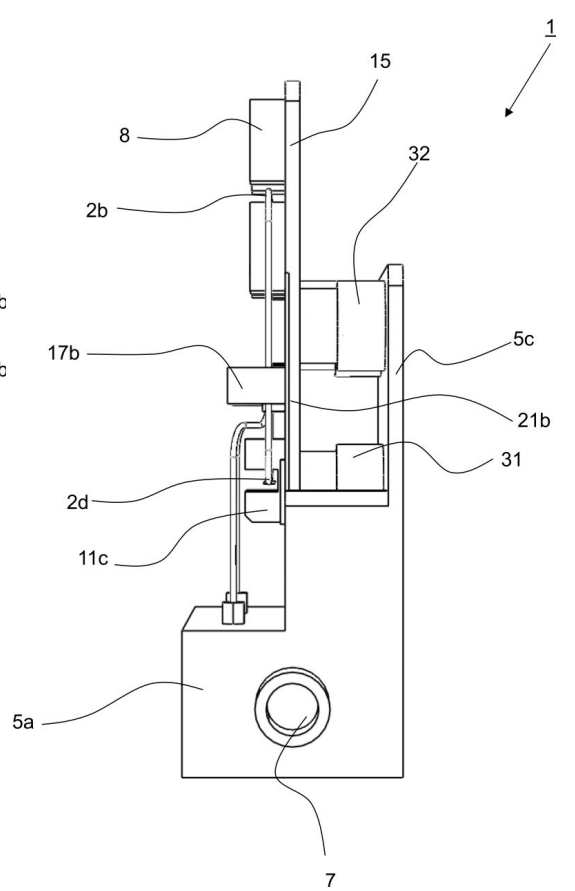

(b)

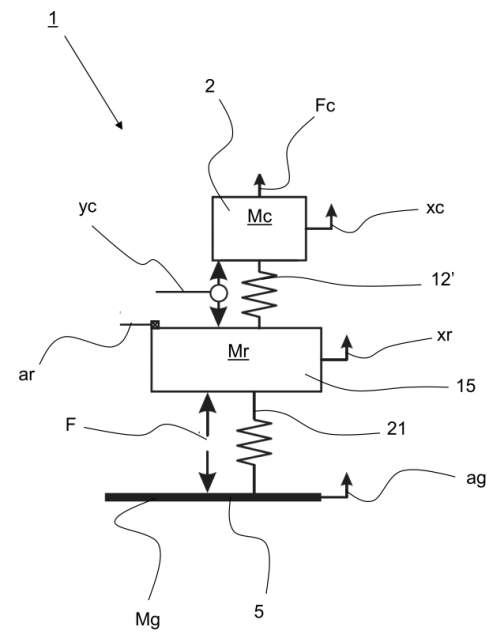

(c)

Fig. C.1 A perspective view, a side view and a dynamical overview of a first embodiment of the flowmeter according to the invention 
In the construction of Figs. C.1(a) and C.1(b) the excitation means for causing the loop 2 to oscillate about the main axis of symmetry $S$ (the primary or excitation axis of rotation) comprise a permanent magnet yoke 19 fastened to the reference mass 15, said yoke having two gaps 9 and 10 through which portions $2 \mathrm{a}$ and $2 \mathrm{~b}$ (denoted the second transverse tubes above) of the looped tube 2 are passed, as well as means for introducing an electric current into the tube 2 . These are means for inducing a current in the tube 2 in the present case. The permanent magnet yoke 8 may, in an alternative embodiment, be attached to the housing 5 as well.

The current is induced in the tube by means of two transformer cores $17 \mathrm{a}, 17 \mathrm{~b}$ provided with respective coils (not shown) through which cores the respective tube portions $2 \mathrm{~d}$ and $2 \mathrm{e}$ are passed. The combination of the magnetic fields generated in the gaps 9 and 10 of the permanently magnetic yoke 8 , which fields are transverse to the direction of the current and are oppositely directed, and an (alternating) current induced in the tube 2 exerts a torque on the tube owing to which it starts to oscillate or rotate about the axis S (in the so-termed twist mode). When a medium flows through the tube, the tube will start to rotate about an axis $S$ ' transverse to the axis $S$ (in the so-termed swing mode) under the influence of Coriolis forces. During operation the (sinusoidal) displacements of points of the tube portion $2 \mathrm{c}$, which are representative of the flow, are detected by detection means 11 in the form of a first sensor 11a and a second sensor $11 \mathrm{~b}$, and optionally a third sensor 11c. The first and the second sensor are arranged on either side of the first axis of rotation S. A third sensor 11c may serve for correction purposes. The sensors may be, for example, of an electromagnetic, inductive, capacitive, or ultrasonic type. In the present case, however, optical sensors are chosen. The sensors 11a, 11b, and 11c each comprise, in the embodiment shown, a U-shaped housing that is fastened to the frame 15, with a light source (for example an LED) placed in the one leg and a photosensitive cell (for example a phototransistor) opposite the light source in the other leg. The lateral tube $2 \mathrm{c}$ is capable of moving between the legs of the U-shaped sensor housings $11 \mathrm{a}$ and $11 \mathrm{~b}$ (and $11 \mathrm{c}$, if present). It should be noted that other ways of detecting displacement (or at least a measure of displacement) of the Coriolis tube are conceivable as well.

The configuration of the Coriolis tube, the excitation means and the detection means described above is per se known to those skilled in the art, and is, for example, described in EP1719982A1 [45], which document is incorporated by reference here.

It can furthermore be seen in Fig. C.1(a) that the frame 15 or reference mass 15 (to which the Coriolis tube 2 is connected by means of fastening means 12) is connected to a main body $5 \mathrm{~b}$ of the casing 5 by means of suspension means 21 . The suspension means 21 comprise a total of three suspension elements $21 \mathrm{a}, 21 \mathrm{~b}, 21 \mathrm{c}$, preferably in the form of flexible beams 21a, 21b, 21c, which may in an embodiment be flexures or spring-leafs, although alternative arrangements are conceivable. The frame 15 is thus resiliently connected to the casing 5 , and the Coriolis tube 2 is resiliently suspended to the frame 15 due to its internal elasticity.

On the frame 15, further detection means 13,14, 16 are provided, which are arranged for detecting vibrations of the frame 15 . These further detection means may be arranged for measuring either position, acceleration, velocity, force, or any other quantity that represents a measure of vibration of the frame 15 . 
As can be seen in Fig. C.1(b), the flowsensor 1 further comprises a number of further excitation means 31,32 , which are connected between a back wall $5 \mathrm{c}$ of the casing 5 , and the frame 15. The excitation means 31,32, may be Piezo actuators, or other actuators. In an embodiment, voice-coil motors are used. In the embodiment shown, a total of three actuators 31,32 are used (one of which is positioned behind the upper actuator 32. The three actuators 31,32 are in the embodiment shown, positioned in a isosceles triangular relationship. It is noted however, that in principle any other number of actuators may be used, and that their position may be chosen as desired.

The flowmeter 1 of the Coriolis type as depicted in Fig. C.1(a) and C.1(b) and described above, can be represented by the dynamical scheme of the flowmeter 1 shown in Fig.C.1(c) Corresponding numbers are adhered to. Here it can be seen that the casing 5 represents 'ground' $M_{\mathrm{g}}$. The frame 15 represents a reference mass $M_{\mathrm{r}}$, and is resiliently connected to the 'ground' $M_{\mathrm{g}}$ by means of the suspension means 21 . To the reference mass $M_{\mathrm{r}}$, the Coriolis flow tube 2 is connected by means of a resilient connection 12'. As described above, the internal elasticity of several tube parts 3, 4, 2a, 2b represents the resilient suspension 12' between the Coriolis mass $M_{\mathrm{c}}$ and the reference mass $M_{\mathrm{r}}$.

The flowmeter of the Coriolis type as shown in Fig. C.1(a)- Fig.C.1(c), operates according to a first principle of active vibration isolation, in order to minimize the effect external vibrations have on the Coriolis-tube. In the embodiment shown, the Coriolis-tube $2\left(M_{\mathrm{c}}\right)$, the reference mass $15\left(M_{\mathrm{r}}\right)$, and the housing (5) are connected in series. The further excitation means 31 connected to the reference mass 15 are used to counter movements ag of the housing 5 so as to substantially fix the absolute position of the reference mass 15 . In other words a stable ground is created for the Coriolis-tube 2, yielding that the measurement $y_{\mathrm{c}}$ is the true movement of the Coriolis tube induced by the Coriolis Force $F_{\mathrm{c}}$ (see Fig. C.1(c)). Hence, vibrations or other disturbances on the housing 5 do not result in undesired vibrations on the Coriolis-tube 2. In general, a feedback scheme and/or a feedforward scheme is/are used to substantially fix the absolute position of the reference mass 15. At least one actuator element, and at least one sensor element are used for this. In the embodiment shown, a total of three actuator elements 31,32 are used.

Referring to Fig. C.1(c), the sensors 11 are arranged, in an embodiment, for measuring a relative displacement $y_{\mathrm{c}}=x_{\mathrm{c}}-x_{\mathrm{r}}$. The displacement of the Coriolis tube due to a Coriolis force $F_{\mathrm{c}}$ (which is proportional to fluid flow) and due to external vibrations is equal to $x_{\mathrm{c}}=G_{1} F_{\mathrm{c}}+G_{2} a_{\mathrm{r}}$, in which $G_{1}$ and $G_{2}$ denote specific transfer functions. The sensors 11 thus actually measure $y_{\mathrm{c}}=x_{\mathrm{c}}-x_{\mathrm{r}}=G_{1} F_{\mathrm{c}}+G_{2} a_{\mathrm{r}}-x_{\mathrm{r}}$. By means of a feedforward and/or a feedback control scheme, the position of the reference mass $15\left(M_{\mathrm{r}}\right)$ may be actively set to an absolute value in which $a_{\mathrm{r}}=x_{\mathrm{r}}=0$. With this, the measurements $y_{\mathrm{c}}$ obtained by the sensors 11 has become dependent on the flow only: $y_{\mathrm{c}}=G_{1} F_{\mathrm{c}}$.

An alternative embodiment of the flowmeter 101 of the Coriolis type according to the invention, is shown in Fig.C.2(a) C.2(c) In general, the same reference signs, increased by 100, are used. In general, it is referred to the description of Fig. C.1(a) and C.1(b), which largely applies to this embodiment. For completeness sake, a short summary will be given below.

Fig. C.2(a) shows a perspective view of a flowmeter 101 of the Coriolis type, and Fig. C.2(b) shows a side view of said flowmeter 101, according to a second embodiment

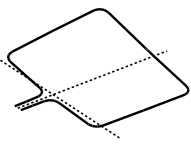




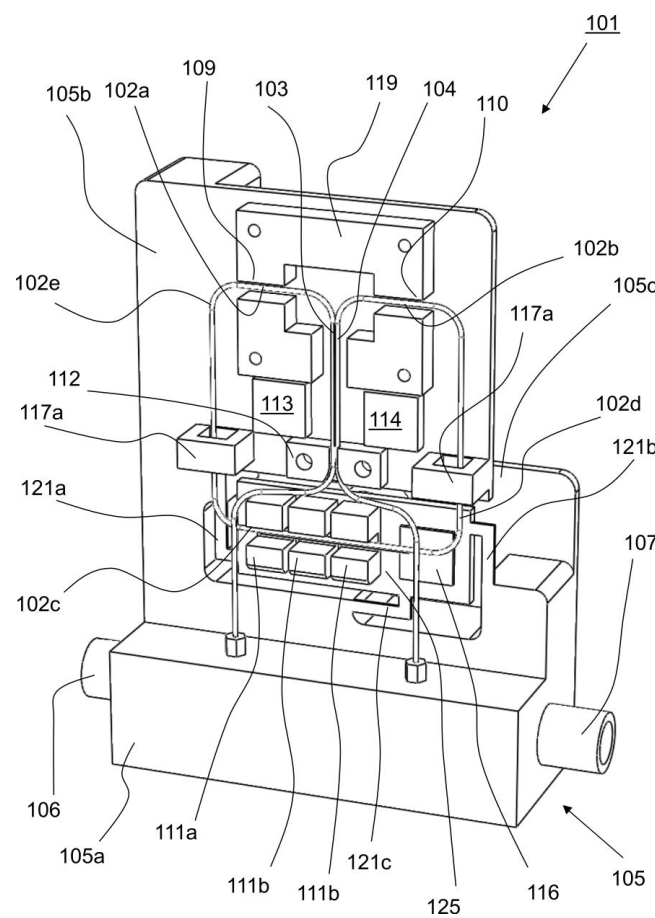

(a)

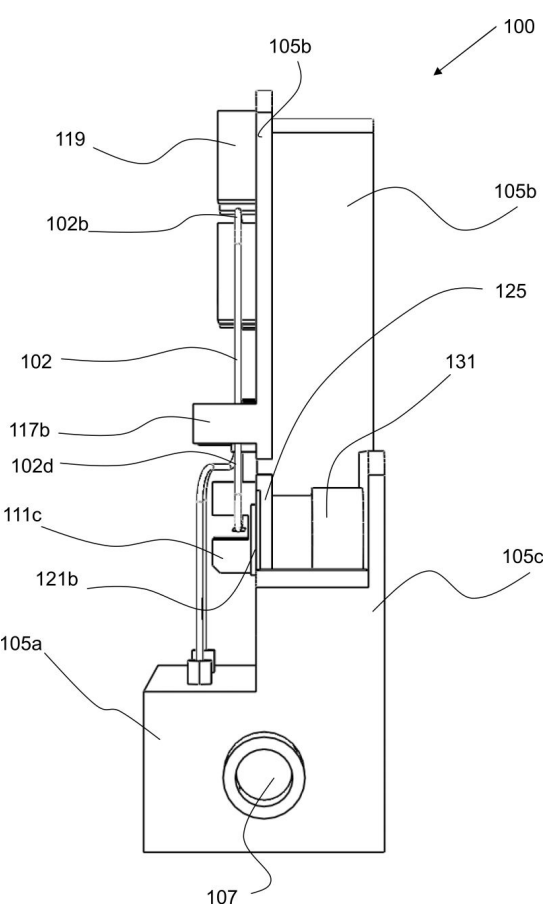

(b)

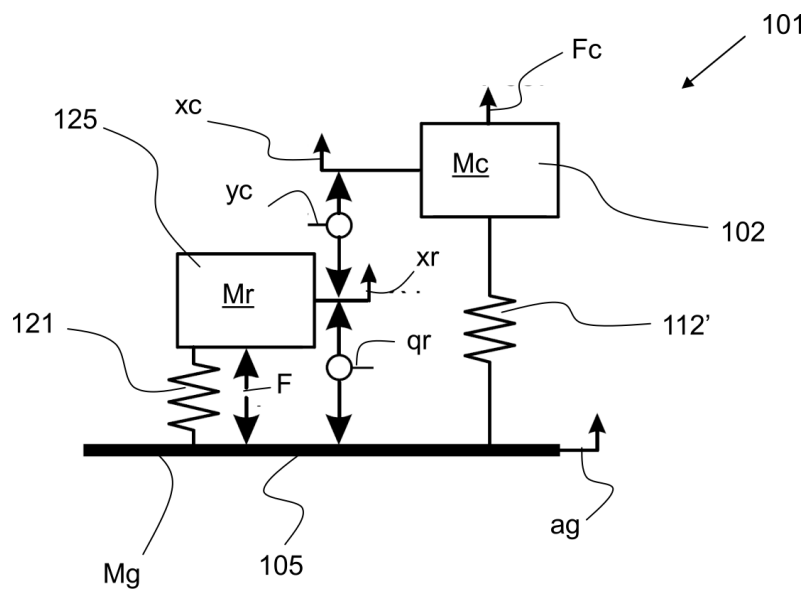

(c)

Fig. C.2 A perspective view, a side view and a dynamical overview of a second embodiment of the flowmeter according to the invention

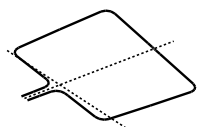


of the invention. The flowmeter 101 of the Coriolis type has a looped tube 102 that is bent into a rectangular shape, with a flexible inlet tube 103 and a flexible outlet tube 104 for a fluid medium. The inlet tube 103 is connected to a supply line 106 and the outlet tube 104 to a discharge line 107 via a supply/discharge block 105a, which forms part of a casing 105 . The tube 102 comprises two parallel lateral tubes $102 \mathrm{~d}$ and $102 \mathrm{e}$, a first transverse tube 102c connected to first (lower) ends of the lateral tubes 102d and 102e, and two second transverse tubes $102 \mathrm{a}$ and $102 \mathrm{~b}$ connected at one side to second (upper) ends of the lateral tubes and at the other side to the centrally returning inlet and outlet tubes 103 and 104, respectively.

Excitation means 119 are provided, comprising a permanent magnet yoke fastened to the main body $105 \mathrm{~b}$ of the casing 105, said yoke having two gaps 109 and 110 through which portions $102 \mathrm{a}$ and $102 \mathrm{~b}$ (denoted the second transverse tubes above) of the looped tube 102 are passed. Transformer cores $117 \mathrm{a}, 117 \mathrm{~b}$ are provided for inducing a current, to exert a torque on the tube owing to which it starts to oscillate or rotate about the axis $\mathrm{S}$ (in the sotermed twist mode). Detection means 111 are provided, in the form of a first sensor 111a and a second sensor $111 \mathrm{~b}$, and optionally a third sensor $111 \mathrm{c}$.

In the embodiment shown, the tubes 103 and 104, are fastened to the fastening means 112 , for example by clamping or soldering or welding, said means 112 in their turn being fastened to the main body $105 \mathrm{~b}$ of the casing 105 . Note, in this sense, that the fastening means 112 are directly connected to the main body $105 \mathrm{~b}$ of the casing 105 .

Connected to the main body $105 \mathrm{~b}$ of the casing 105 is further frame 125 . The frame 125 is resiliently connected, by means of suspension means 121 , which comprise in the embodiment shown a total of three suspension elements 121a, 121b, 121c in the form of flexible beams, preferably flexure elements or spring-leaf like elements. Further detection means 116 are provided, which are arranged for determining vibrations of the frame 125.

As can be seen in Fig. C.2(c), a further excitation means 131 is provided, which may be used to excite the frame 125.

Fig. C.2(c) shows a dynamical scheme of the embodiment of the flowmeter 101 as shown in Fig. C.2(a) and C.2(b) Here it can be seen that the frame 125 defines a reference mass $M_{\mathrm{r}}$, which is resiliently connected by means of the suspension means 121 to the 'ground mass' formed by the casing 105 . The Coriolis flow tube 102 defines a Coriolis mass $M_{\mathrm{c}}$, and is directly connected, in a resilient manner, by means of a resilient suspension 112' (formed by internal elasticity of one or more of the Coriolis tube parts) to the 'ground mass' formed by the casing 105 . Thus, the reference mass 125 and the Coriolis tube 102 are connected in parallel to each other.

The embodiment of the flowmeter 101 of the Coriolis type as shown in Fig. C.2(a) C.2(c) works on a second principle of active vibration isolation. In this second principle, the Coriolis-tube $102\left(M_{\mathrm{c}}\right)$ and the reference mass $125\left(M_{\mathrm{r}}\right)$ are positioned in parallel to each other, each being connected to the housing 105. The further excitation means 131 are used to match the dynamic properties of the reference mass 125 with the dynamic properties of the Coriolis-tube 102. Disturbances due to external vibrations acting on the housing 105 act on both the reference mass 125 and the Coriolis-tube 102, in a similar manner. Thus, the relative displacements of the Coriolis tube are at least substantially dependent on the Coriolis force and independent on the external vibrations. It is noted in this regards that the sensors elements 111a-111c of the detection means 111 are in this embodiment provided on the reference mass 
125 , such that the sensor elements $111 \mathrm{a}-111 \mathrm{c}$ are able to measure the Coriolis force induced vibrations $x_{\mathrm{c}}$ on the Coriolis-tube.

In the embodiment shown in Fig.C.2(c), the sensors 111 are arranged for measuring a relative displacement $y_{\mathrm{c}}=x_{\mathrm{c}}-x_{\mathrm{r}}$. Displacement $y_{\mathrm{c}}$ of the Coriolis tube, due to a Coriolis force $F_{\mathrm{c}}$ (which is proportional to fluid flow) and due to external vibrations equals $x_{\mathrm{c}}=G_{1} F_{\mathrm{c}}+G_{3} a_{\mathrm{g}}$; with $G_{1}$ and $G_{3}$ being specific transfer functions. Now in this embodiment, movement of the reference mass $M_{\mathrm{r}}$ equals $x_{\mathrm{r}}=G_{4} a_{\mathrm{g}}$. With this, the sensors 111 are arranged for measuring $y_{\mathrm{c}}=x_{\mathrm{c}}-x_{\mathrm{r}}=G_{1} F_{\mathrm{c}}+\left(G_{3}-G_{4}\right) a_{\mathrm{g}}$. The measurement of the sensors 111 is dependent on the fluid flow only, when $G_{3}$ equals $G_{4}$ (in other words, when dynamic properties of the reference mass match those of the Coriolis tube). A feedback and/or feedforward control scheme may be used to achieve this.

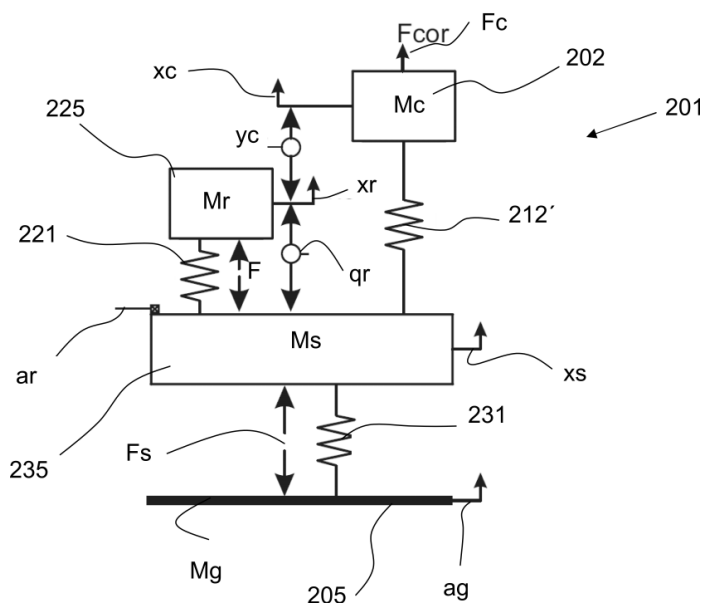

Fig. C.3 A dynamical overview of a third embodiment of the flowmeter according to the invention

Fig. C.3, shows a dynamic representation of a further embodiment of the flowmeter 201 according to the invention. This flowmeter 201 makes use of the first and second principle described above. The flowmeter 201 comprises, a housing $205\left(M_{\mathrm{g}}\right)$. Connected thereto, is an additional reference mass $235\left(M_{\mathrm{s}}\right)$. The additional reference mass 235 is resiliently connected to the housing 205, by means of one or more spring elements 231 . Connected to the additional reference mass 235 are a reference mass $225\left(M_{\mathrm{r}}\right)$ and a Coriolis tube $202\left(M_{\mathrm{c}}\right)$. The Coriolis tube 202 is resiliently suspended due to internal elasticity, which is denoted by reference sign $212^{\prime}$. The Coriolis tube 202 and the reference mass 225 are connected in parallel to each other. Both the reference mass 225 and the Coriolis tube 202 are resiliently connected to the additional reference mass 235 . As can be seen, this embodiment is basically a combination of Fig. C.2(c) and Fig. C.1(c).

The flowmeter 201 makes use of a combined principle of active vibration isolation, in this sense that the additional reference mass uses additional excitation means (not shown) and additional detection means to detect vibrations and/or movements of the housing 205, and to

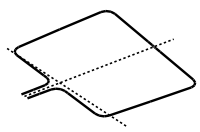


substantially fix the absolute position xs of the additional reference mass. This provides a stable base for the Coriolis tube 202. Then the reference mass 225 with further excitation means and further detection means may be used to match the dynamic properties of the reference mass 225 to the dynamic properties of the Coriolis tube 202, such that any disturbances acting on the housing (and which are not countered by the additional mass) or on the reference mass 235 have the same effect on the reference mass 225 and the Coriolis tube, and the Coriolis displacement may be more accurately measured.

According to the embodiment shown in Fig. C.3 the sensors 211 are arranged for measuring a relative displacement $y_{\mathrm{c}}=x_{\mathrm{c}}-x_{\mathrm{r}}$. The displacement of the Coriolis tube due to a Coriolis force (which is proportional to fluid flow) and due to external vibrations equals $x_{\mathrm{c}}=G_{1} F_{\mathrm{c}}+G_{5} x_{\mathrm{s}}$. In this case, the displacement of the reference mass equals $x_{\mathrm{r}}=G_{6} x_{\mathrm{s}}$. Here, the sensors 211 are thus arranged for measuring $y_{\mathrm{c}}=G_{1} F_{\mathrm{c}}+\left(G_{5}-G_{6}\right) x_{\mathrm{s}}$. The dynamical properties of the reference mass may be matched to those of the Coriolis tube, such that $G_{5}=G_{6}$. The reference mass may be set to an absolute position, using a feedback and/or feedforward control scheme, such that $a_{\mathrm{s}}=x_{\mathrm{s}}=0$. This leads to the result that the measurement depends only on the fluid flow; $y_{\mathrm{c}}=G_{1} F_{\mathrm{c}}$.

Fig. C.4(a) shows a perspective view of a flowmeter 301 of the Coriolis type (or Coriolis Mass Flow Meter, CMFM), and Fig. C.4(b) shows a side view of said flowmeter 301, according to an embodiment of the invention wherein passive vibration isolation is incorporated into the Coriolis flow sensor. As already described for Fig.C.1(a) and C.1(b), the flowmeter 301 of the Coriolis type has a looped tube 302 that is bent into a rectangular shape so as to follow a substantially circumferential path (substantially one full turn), and that comprises a flexible inlet tube 303 and a flexible outlet tube 304 for a fluid medium. Preferably, the loop 302 and the inlet and outlet tubes 303, 304 are portions of one and the same tube. The tube 302 in its entirety is bent into a rectangular shape, but the corners are rounded such that it can be bent into this shape. The inlet tube 303 is connected to a supply line 306 and the outlet tube 304 to a discharge line 307 via a supply/discharge block 305a, which forms part of a casing 305. The inlet and outlet tubes 303, 304 of this embodiment extend within the loop 302 and are fastened (i.e. clamped) to the casing 305 by fastening means 312 . The fastening is provided in a location such that the free path length of the inlet and the outlet tube 303, 304 (i.e. the portion of the inlet/outlet tube 303, 304 between the connection of the second transverse tube portions $302 \mathrm{a}, 302 \mathrm{~b}$ and the location of the clamping to the fastening means 312 ) is at least $50 \%$, preferably $60 \%$ of the length of each of the lateral tube portions $302 \mathrm{c}$, $302 \mathrm{~d}$, or even longer. The flexible inlet and outlet tubes 303, 304 do not form part of the loop 302 but provide a flexible fastening of the loop 302 to the casing 305. The loop 302 may thus be regarded as being flexibly suspended by means of the inlet and outlet tubes. The loop 302 and the inlet and outlet tubes 303, 304 may advantageously be manufactured from one integral piece of tubing. This may be, for example, a stainless steel tube with an outer diameter of approximately $0.7 \mathrm{~mm}$ and a wall thickness of approximately $0.1 \mathrm{~mm}$. Depending on the outer dimensions of the loop 302 and the pressure the tube is to be able to withstand (for example 100 bar), the outer diameter of the tube will usually be smaller than $1 \mathrm{~mm}$ and the wall thickness $0.2 \mathrm{~mm}$ or less. It is noted that other dimensions may be used as well.

The tube 302 consists of a substantially rectangular framework comprising two parallel lateral tubes $302 \mathrm{~d}$ and 302e, a first transverse tube 302c connected to first (lower) ends of

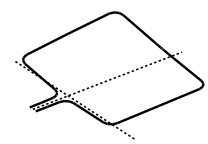




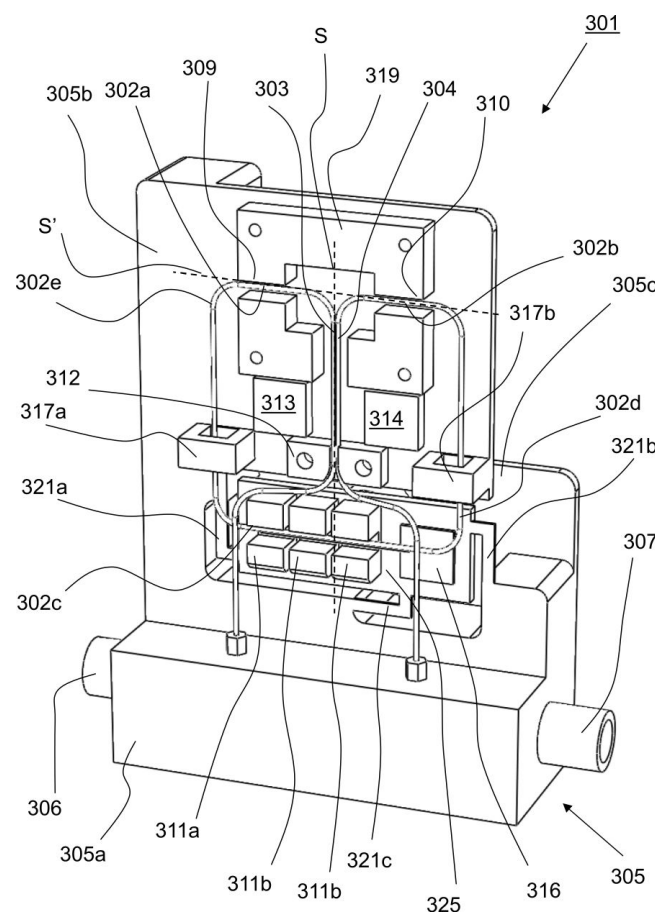

(a)

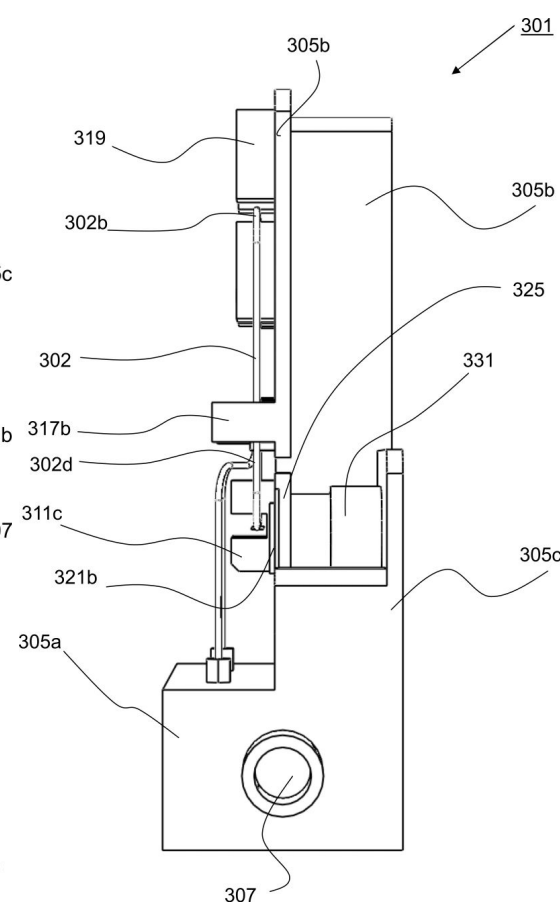

(b)

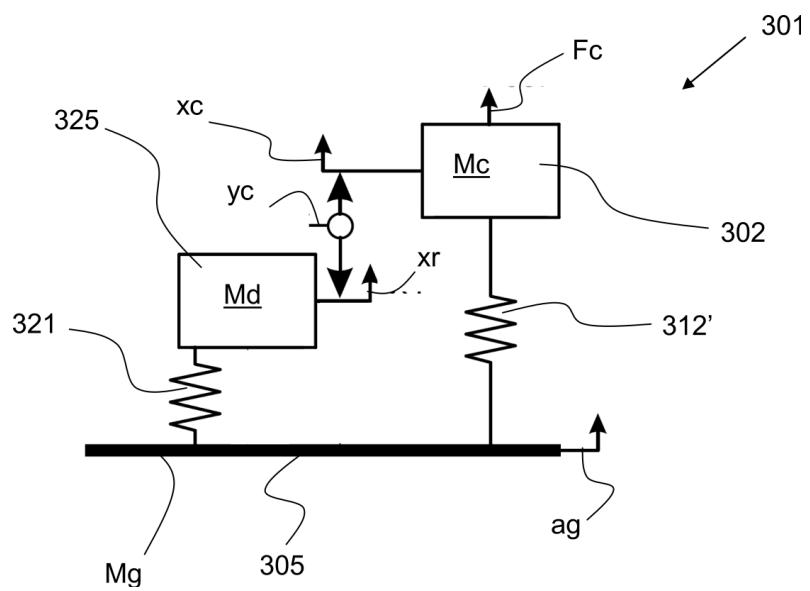

(c)

Fig. C.4 A perspective view, a side view and a dynamical overview of a third embodiment of the flowmeter according to the invention

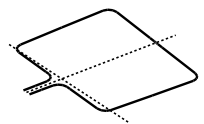


the lateral tubes $302 \mathrm{~d}$ and 302e, and two second transverse tubes 302a and 302b connected at one side to second (upper) ends of the lateral tubes and at the other side to the centrally returning inlet and outlet tubes 303 and 304, respectively. The rectangular loop 302 preferably has rounded corners. The tubes 303 and 304, which run closely together on either side of and symmetrically with respect to the main axis of symmetry $\mathrm{S}$ of the loop 302, are fastened to the fastening means 312 , for example by clamping or soldering or welding, said means 312 in their turn being fastened to the casing 305. The inlet and outlet tubes 303, 304 are flexible and act as it were as a suspension spring for the loop 302. This suspension allows a motion of the loop 302 both about the main axis of symmetry $S$ and about a second axis S' situated in the plane of the loop 302 and perpendicular to the main axis of symmetry S.

To close the loop 302 mechanically (i.e. to interconnect the beginning and end of the loop mechanically, directly or indirectly), the tubes 303, 304 are preferably connected to one another along the extent of their free path lengths, for example in that they are welded or soldered together.

In the construction of Figs. C.1(a) and C.1(b) the excitation means for causing the loop 302 to oscillate about the main axis of symmetry S (the primary or excitation axis of rotation) comprise a permanent magnet yoke 319 fastened to the casing 305, said yoke having two gaps 309 and 310 through which portions 302a and 302b (denoted the second transverse tubes above) of the looped tube 302 are passed, as well as means for introducing an electric current into the tube 302. These are means for inducing a current in the tube 302 in the present case.

The current is induced in the tube by means of two transformer cores $317 \mathrm{a}, 317 \mathrm{~b}$ provided with respective coils (not shown) through which cores the respective tube portions $302 \mathrm{~d}$ and $302 \mathrm{e}$ are passed. The combination of the magnetic fields generated in the gaps 309 and 310 of the permanently magnetic yoke 319 , which fields are transverse to the direction of the current and are oppositely directed, and an (alternating) current induced in the tube 302 exerts a torque on the tube owing to which it starts to oscillate or rotate about the axis $\mathrm{S}$ (in the sotermed twist mode). When a medium flows through the tube, the tube will start to rotate about an axis $S^{\prime}$ transverse to the axis $S$ (in the so-termed swing mode) under the influence of Coriolis forces. During operation the (sinusoidal) displacements of points of the tube portion $302 \mathrm{c}$, which are representative of the flow, are detected by detection means 311 in the form of a first sensor 311a and a second sensor 311b, and optionally a third sensor 311c. The first and the second sensor are arranged on either side of the first axis of rotation S. A third sensor 311c may serve for correction purposes. The sensors may be, for example, of an electromagnetic, inductive, capacitive, or ultrasonic type. In the present case, however, optical sensors are chosen. The sensors $311 \mathrm{a}, 311 \mathrm{~b}$, and 311c each comprise, in the embodiment shown, a Ushaped housing that is fastened to the frame 315, with a light source (for example an LED) placed in the one leg and a photosensitive cell (for example a phototransistor) opposite the light source in the other leg. The lateral tube $302 \mathrm{c}$ is capable of moving between the legs of the Ushaped sensor housings 311a and 311b (and 311c, if present). It should be noted that other ways of detecting displacement (or at least a measure of displacement) of the Coriolis tube are conceivable as well.

In the embodiment shown, the tubes 303 and 304, are fastened to the fastening means 312 , for example by clamping or soldering or welding, said means 312 in their turn being fastened

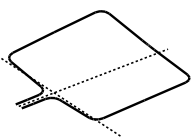


to the main body $305 \mathrm{~b}$ of the casing 305 . Note, in this sense, that the fastening means 312 are directly connected to the main body $305 \mathrm{~b}$ of the casing 305 .

Connected to the main body $305 \mathrm{~b}$ of the casing 305 is further frame 325 . The further frame 325 is resiliently connected, by means of suspension means 321 , which comprise in the embodiment shown a total of three suspension elements 321a, 321b, 321c in the form of flexible beams, preferably flexure elements or spring-leaf like elements. On the further frame 325, the detection means 311 are provided.

According to an aspect of the invention, which is in particular related to passive vibration isolation, a compliant connection 321 between the casing 305 and the detection means 311 is present. The compliant connection 321 according to the invention is constructed and arranged such that a relative displacement measurement of the tube 302, dependent on the tube 302 actuation and mass-flow, though substantially independent of casing 305 excitations is obtained. In other words, the further frame 325 with the detection means 311 is arranged to oscillate, in response to casing 305 excitations, in substantially the same manner as the Coriolistube 302 oscillates in response to said same casing 305 excitations. Thus, the influence of external casing excitations does not result in a relative movement between the Coriolis-tube 302 and the detection means 311 .

Fig. C.4(c) shows a dynamical scheme of the embodiment of the flowmeter 301 as shown in Fig.C.4(a) and C.4(b) Here it can be seen that the frame 325 with the detection means 311 is resiliently connected by means of the suspension means 321 to the 'ground mass' formed by the casing 305 . The Coriolis flow tube 302 defines a Coriolis mass $M_{\mathrm{c}}$, and is directly connected, in a resilient manner, by means of a resilient suspension 312' (formed by internal elasticity of one or more of the Coriolis tube parts) to the 'ground mass' formed by the casing 305. Thus, the detection means 325 and the Coriolis tube 302 are connected in parallel to each other.

The placement and the characteristics of the compliant connection 321, as well as design of the flow sensor (including design for masses $M_{\mathrm{d}}$ and $M_{\mathrm{c}}$ of the frame 325 with detection means, and the Coriolis tube, respectively, may be designed in order to obtain the desired effect. In particular, the compliant connection 321 is arranged and designed such that the resonance frequency and/or the damping of the compliant detection means 311 are tuned such that the dynamic response to external vibrations on the casing is substantially equal to said response of the Coriolis tube.

As followed from the above, the Coriolis flow-sensor thus comprises passive vibration isolation means. In the embodiment shown in Fig. C.4(a) and C.4(b), the Coriolis flow-sensor also comprises active vibration isolation means, such as described for Figs.C.1.C.3, although these means are entirely optional. In summary, referring to Fig. C.4(a) and C.4(b), a further excitation means 331 is provided, which may be used to actively excite the further frame 325 as a reference mass, and further detection means 316 are provided, to measure the oscillations of the reference mass.

The Coriolis-tube 302 (having mass $M_{\mathrm{c}}$ ) and the reference mass $M_{\mathrm{d}}$ provided by the frame 325 are positioned in parallel to each other, each being connected to the housing 305 . The further excitation means 331 are used to match the dynamic properties of the reference mass 325 with the dynamic properties of the Coriolis-tube 302. Disturbances due to external vibrations acting on the housing 305 act on both the reference mass 325 and the Coriolis-tube 302, in a

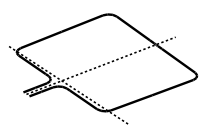


similar manner. Thus, the relative displacements of the Coriolis tube are at least substantially dependent on the Coriolis force and independent on the external vibrations. It is noted in this regards that the sensors elements 311a-311c of the detection means 311 are in this embodiment provided on the reference mass 325, such that the sensor elements 311a-311c are able to measure the Coriolis force induced vibrations $x_{\mathrm{c}}$ on the Coriolis-tube 302.

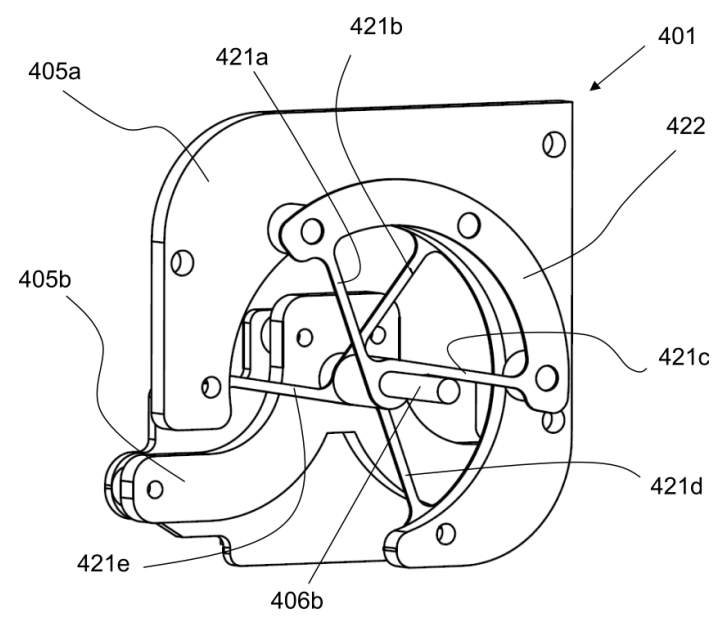

Fig. C.5 An embodiment of a compliant sensor design for passive vibration isolation according to an aspect of the invention

An embodiment of a device 401 having passive vibration isolation is shown in Fig. C.5 The device 401 comprises a casing part $405 \mathrm{a}$ and a frame part $405 \mathrm{~b}$. The frame part $405 \mathrm{~b}$ has a compliant connection 421 to the casing part 405a. In the embodiment shown, several wire springs 421a-421e are used, which are arranged in such a manner to provide an exactly constraint configuration with only one remaining degree of freedom, which is along the longitudinal length of cylinder 406b. The device 401 may be used in a flow sensor of the Coriolis type, wherein the Coriolis tube is connected to the casing 405a, and detections means are provided on the frame part 405b, such that the detection means are resiliently connected to the casing. The Coriolis tube is provided in such a way that the only remaining degree of freedom is out of plane of the tube-window. This results in an extra degree of freedom between the casing $405 \mathrm{a}$ and the frame $405 \mathrm{~b}$, which preferably comprises a printed circuit board (PCB) with the detection means in the form of optical sensors.

The resilient connection, in the embodiment shown provided by flexures, or leaf springs, provides damping and stiffness. The connection of the Coriolis tube to the casing also entails damping and stiffness. Perfect vibration isolation may be achieved when the following conditions are met:

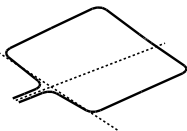


- the damping of the detection means $d_{\mathrm{d}}$ is equal to damping of the Coriolis tube $d_{\mathrm{c}}$ times the ratio between the mass of the detection means $m_{\mathrm{d}}$ and the mass of the Coriolis tube $m_{\mathrm{c}}: d_{\mathrm{d}}=\frac{m_{\mathrm{d}}}{m_{\mathrm{c}}} d_{\mathrm{c}}$

- the stiffness of the detection means $k_{\mathrm{d}}$ is equal to stiffness of the Coriolis tube $k_{\mathrm{c}}$ times the ratio between the mass of the detection means $m_{\mathrm{d}}$ and the mass of the Coriolis tube $m_{\mathrm{c}}: k_{\mathrm{d}}=\frac{m_{\mathrm{d}}}{m_{\mathrm{c}}} k_{\mathrm{c}}$.

These conditions are in general achieved when the resonance frequency and the damping ratio are equal for the internal mode of the tube window and the compliant mounted detection means.

It is noted that for the design shown in Fig. C.5 a perfect match of the damping ratio and the resonance frequency no longer results in perfect vibration isolation because of the higher order dynamics of the tube window. Fortunately, mistuning of those parameters can be used advantageously to minimise the transmissibility in the region of interest by the introduction of an anti-resonance. Therefore the concept device 401 shown in Fig. C.5 is mistuned on purpose.

In the case shown in Fig. C.5, the damping and resonance frequencies for the internal mode of the tube-window (Coriolis-tube) are approximately $90 \%$ of those of the compliant mounted detection means (frame 405b). With the design, a $20 \mathrm{~dB}$ attenuation of the influence of external vibrations on the mass-flow measurement value of a CMFM may be obtained.

The device 401 shown in Fig. C.5 -as well as concepts described above in relationship to Coriolis flow sensors- can in principle be used in every flow sensor of the Coriolis type, and even in other kinds of systems were specific internal deformations need to be measured independently of external vibrations.

In particular, the passive vibration isolation described in the embodiments of Figs. C.4 and C.5 can be incorporated into the Coriolis flow sensors as described in Figs. C.1 to C.3

The invention is described above by means of a number of embodiments. These embodiments and the description thereof are not to be construed limiting on the invention. The invention and its desired protection is defined by the appended claims.

The Coriolis flow sensor having passive vibration isolation may be described by means of the following clauses:

Clause 1. Coriolis flow sensor, comprising a housing and at least a Coriolis- tube with at least two ends being fixed in a tube fixation means, wherein the flow sensor comprises excitation means for causing the tube to oscillate, as well as detection means for detecting at least a measure of displacements of parts of the tube during operation, characterized in that it comprises a compliant connection between the casing and at least part of the detection means.

Clause 2. Coriolis flow sensor according to clause 1, wherein the flow sensor is arranged for causing the detection means to oscillate in response to casing excitations, in substantially the same manner as the Coriolis-tube oscillates in response to casing excitations.

Clause 3. Coriolis flow sensor according to clause 2, wherein the resonance frequency and/or the damping ratio are substantially equal for the Coriolis-tube and the compliant mounted detection means. 
Clause 4. Coriolis flow sensor according to clause 2, wherein the resonance frequency and/or the damping ratio are lower for the internal mode of the tubewindow, compared to the resonance frequency and the damping ratio of the compliant mounted detection means.

Clause 5. Coriolis flow sensor according to clause 4, wherein the resonance frequency and/or the damping ratio for the Coriolis-tube are approximately $90 \%$ of the resonance frequency and the damping ratio of the compliant mounted detection means.

Clause 6. Coriolis flow sensor according to clause 2, wherein the resonance frequency and/or the damping ratio is/are higher for the internal mode of the Coriolis- 5 tube, compared to the resonance frequency and the damping ratio of the compliant mounted detection means.

Clause 7. Coriolis flow sensor according to any of the previous clauses, additionally comprising the features of the Coriolis flow sensor according to any of the following claims $1-20$.

\section{C.2 Claims}

1. Coriolis flow sensor, comprising a housing and at least a Coriolis- tube with at least two ends being fixed in a tube fixation means, wherein the flow sensor comprises excitation means for causing the tube to oscillate, as well as detection means for detecting at least a measure of displacements of parts of the tube during operation, characterized in that it comprises:

- a reference mass;

- further excitation means arranged for causing the reference mass to oscillate with respect to the housing;

- further detection means arranged for detecting at least a measure of displacements of the reference mass and/or the housing during operation; as well as

- control means for controlling the excitation means and/or the further excitation means based on movements measured by the detection means and/or further detection means.

2. Coriolis flow sensor according to claim 1, wherein the reference mass is connected to the housing.

3. Coriolis flow sensor according to claim 2, wherein the reference mass is connected to the housing by means of spring elements, in particular flexures or spring-leafs.

4. Coriolis flow sensor according to one of claims 1-3, wherein the reference mass comprises the tube fixation means, or at least wherein the tube fixation means are substantially rigidly connected to the reference mass.

5. Coriolis flow sensor according to one of claims 1-4, wherein the control means are arranged for moving the reference mass in opposite directions to movements of the housing, in particular movements due to external vibrations acting on the housing. 
6. Coriolis flow sensor according to one of claims 1-5, wherein the further excitation means are provided between the housing or a part rigidly connected to the housing, on the one hand, and to the reference mass on the other hand.

7. Coriolis flow sensor according to one of claims 1-6, wherein the further excitation means comprise at least three excitation members, and wherein the detection means comprise at least three detection members.

8. Coriolis flow sensor according to one of claims 1-7, wherein the further detection means are arranged for detecting at least a measure for movements of the housing or a part rigidly connected thereto.

9. Coriolis flow sensor according to claim 8, wherein the control means are arranged for moving the reference mass with respect to the housing based on feedforward control.

10. Coriolis flow sensor according to one of claims 1-9, wherein the further detection means are arranged for detecting at least a measure for movements of the reference mass, and wherein the control means are arranged for moving the reference mass based on feedback control.

11. Coriolis flow sensor according to claim 9 and 10, wherein the further detection means are arranged for detecting movements of the housing, and wherein additional detection means are provided for detecting movements of the reference mass, and wherein the control means are arranged for moving the reference mass with respect to the housing based on both feedforward control and feedback control.

12. Coriolis flow sensor according to one of claims 1-11, wherein the reference mass comprises the excitation means for the Coriolis tube.

13. Coriolis flow sensor according to one of claims 1-12, wherein the reference mass comprises the detection means for the Coriolis tube.

14. Coriolis flow sensor according to one of claims 1-13, wherein the housing comprises the tube fixation means, or at least wherein the tube fixation means is rigidly connected to the housing

15. Coriolis flow sensor according to claim 14, wherein the control means are arranged for moving the reference mass substantially identically to the Coriolis tube in response to movements of the housing.

16. Coriolis flow sensor according to one of claims 14-15, wherein the control means are arranged for moving the reference mass based on feedback control or on feedforward control, wherein use is made of signals of the further detection means.

17. Coriolis flow sensor according to one of claims 1-13, comprising an additional reference mass connected to the reference mass, additional excitation means arranged for causing the additional reference mass to oscillate, as well as additional detection means for detecting at least a measure for displacements of the additional reference mass during operation.

18. Coriolis flow sensor according to claim 17, wherein the control means further are arranged for moving the additional reference mass substantially identically to the Coriolis tube in response to movements of the reference mass.

19. Coriolis flow sensor according to one of claims 1-18, wherein the detection means and/or further detection means, and/or additional detection means are arranged for measuring 
acceleration, velocity, position, strain and/or force of the Coriolis tube, the reference mass and/or the housing.

20. Coriolis flow sensor according to one of claims 1-19, wherein the excitation means and/or further excitation means, and/or additional excitation means comprise an excitation member in the form of a Lorentz actuator, a Voicecoil motorelement, a reluctance actuator and/or a Piezo element.

21. Coriolis flow sensor according to one of claims 1-20, comprising a compliant connection between the casing and at least part of the detection means.

22. Coriolis flow sensor according to claim 21, wherein the flow sensor is arranged for causing the detection means to oscillate in response to casing excitations, in substantially the same manner as the Coriolis-tube oscillates in response to casing excitations.

23. Coriolis flow sensor according to claim 22, wherein the resonance frequency and/or the damping ratio are substantially equal for the Coriolis-tube and the compliant mounted detection means.

24. Coriolis flow sensor according to claim 22, wherein the resonance frequency and/or the damping ratio are lower for the internal mode of the tubewindow, compared to the resonance frequency and the damping ratio of the compliant mounted detection means.

25. Coriolis flow sensor according to claim 24, wherein the resonance frequency and/or the damping ratio for the Coriolis-tube are approximately $90 \%$ of the resonance frequency and the damping ratio of the compliant mounted detection means.

26. Coriolis flow sensor according to claim 22, wherein the resonance frequency and/or the damping ratio is/are higher for the internal mode of the Coriolistube, compared to the resonance frequency and the damping ratio of the compliant mounted detection means.

27. Method for actively isolating vibrations in a flowsensor of the Coriolis type, preferably in a Coriolis flow sensor according to one of claims 1-26, comprising a Coriolis flow tube and a reference mass that is connected thereto in a flexible manner, wherein the method comprises the step of substantially fixing the position of the reference mass.

28. Method for actively isolating vibrations in a flowsensor of the Coriolis type, preferably in a Coriolis flow sensor according to one of claims 1-26, comprising a Coriolis flow tube and a reference mass that is movable independently thereof, wherein the method comprises the step of substantially matching the dynamical behavior of the reference mass to the dynamical behavior of the Coriolis tube. 


\section{Dankwoord}

Het is af! Na vier jaar werken heb ik de belangrijkste resultaten van mijn promotieonderzoek gepresenteerd in dit proefschrift. Dit heb ik echter niet alleen voor elkaar gekregen. Daarom wil ik iedereen bedanken die de afgelopen jaren bewust of onbewust heeft bijgedragen aan het realiseren van het behaalde resultaat.

In het bijzonder wil ik mijn dagelijkse begeleiders Wouter en Johannes bedanken voor de waardevolle bijdrage, de kritische opmerkingen, de discussies en jullie kennis die zorgden voor de diepgang van mijn onderzoek. Daarnaast wil ik André bedanken voor de tijd en moeite die je hebt gestoken in het begeleiden van mijn promotie.

Ik wil Michel, Kyrian, Kees en Beralt bedanken voor hun inhoudelijke bijdrage aan het onderzoek, de vele discussies en leergierigheid die zorgden voor vele nieuwe inzichten. Leo, bedankt voor het realiseren van de experimentele opstellingen die onmisbaar zijn geweest om de ideeën te kunnen valideren.

Een goede motivatie voor het wetenschappelijke onderzoek, was de blijvende interesse vanuit de industrie. Bronkhorst High Tech, en met name Marcel, Joost en Wim, bedankt voor jullie betrokkenheid, het delen van actuele problemen en de implementatievraagstukken voor nieuwe producten. Daarnaast wil ik ook Demcon bedanken voor de interesse en inhoudelijke kennis van het onderwerp.

Verder wil ik alle collega's van de vakgroep WA bedanken voor hun hulp, interessante discussies en de gezelligheid bij de koffiemomenten en de uitjes. Dit heeft zeker bijgedragen aan het eindresultaat. Mijn vrienden en teamgenoten van Harambee bedankt voor alle gezellige en sportieve momenten naast het werk.

Mijn vader, moeder, broer, zussen en verdere familie wil ik bedanken voor hun vertrouwen, liefde en steun. Zonder deze bron van motivatie was dit resultaat er nooit geweest.

$\mathrm{Na} 10$ jaar aan de universiteit Twente kan ik nu eindelijk zeggen dat ik student-af ben. Het is een einde van een leerzame periode, maar een mooi begin van een nieuwe uitdaging!

Bert van de Ridder

mei 2015, Enschede 
Abstract: A Coriolis Mass-Flow Meter (CMFM) is an active device based on the Coriolis force principle for direct mass-flow measurements, with high accuracy, range-ability and repeatability. The working principle of a CMFM is as follows: a fluid conveying tube is actuated to oscillate at a low amplitude, whereby a resonance frequency is used to minimise the amount of required energy. A fluid-flow in the vibrating tube induces Coriolis forces, which are proportional to the mass-flow, and affect the tube motion resulting in a change of the mode shape. External vibrations create additional components in the CMFM sensor signals and such additional components can introduce a measurement error.

This thesis presents a comprehensive analysis on how narrow-band vibration isolation of more than $\mathbf{4 0} \mathrm{dB}$ can be achieved. The active vibration isolation control is successfully implemented in a design of a CMFM, for the first time.

Bert van de Ridder was born on 22nd of April, 1987 in Putten, The Netherlands. After he finished secondary school in 2005, he started with Mechanical Engineering at the University of Twente. He finished his Bachelor of Science cum laude in 2008. He continued his study in the group of Mechanical Automation to obtain the Master of Science in 2011. In the same group he performed his PhD research and finished it in 2015. The results of the research project are presented in this thesis.
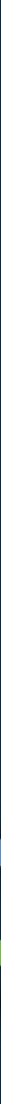

ISBN 978-90-365-3874-9

UNIVERSITY OF TWENTE. 